\title{
AN INVERSE METHOD FOR OBTAINING THE ATTENUATION PROFILE AND SMALL VARLATIONS IN THE SOUND SPEED AND DENSITY PROFLES OF THE OCEAN BOTTOM
}

\author{
by \\ Subramaniam D. Rajan \\ B.E., 1954 University of Madras \\ SUBMITTED IN PARTLAL FULFILLMENT OF THE REQUTREMENTS \\ FOR THE DEGREE OF DOCTOR OF PHILOSOPHY
}

\author{
at the \\ MASSACHUSETTS INSTITUTE OF TECHNOLOGY \\ and the \\ WOODS HOLE OCEANOGRAPHIC INSTITUTION
}

May 1985

Subramaniam D. Rajan

Signature of Author

Department of Ocean Engineering, Massachusetts Institute of Technology and the Joint Program in Oceanography/ Oceanographic Engineering, Massachusetts Iinstitute of Technology/Woods Hole Oceanographic Institution.

Certified by

George V. Frisk, Thesis Supervisor

Accepted by

William D. Grant, Chairman, Joint Committee for Oceanographic Engineering, Massachusetts Institute of Technology/Woods Hole Oceanographic Institution. 
An Inverse Method for Obtaining the Attenuation

Profile and Small Variations in the Sound Speed

and Density Profiles of the Ocean Bottom

\section{Subramaniam D. Rajan}

Submitted in partial fulfillment of the requirements

for the Degree of Doctor of Philosophy

at the

\section{MASSACHUSETTS INSTITUTE OF TECHNOLOGY and the WOODS HOLE OCEANOGRAPHIC INSTITUTION}

May, 1885

\section{ABSTRACT}

The acoustic properties of marine sediments have a direct effect on the propagation of sound in the ocean. In the frequency range of interest $(50-500 \mathrm{~Hz})$ the sediment can be modelled as a fluid. Assuming horizontal stratification of the ocean bottom, the acoustic parameters of interest are the compressional wave speed, the compressional wave attenuation and density as a function of depth.

An inverse method based on a perturbation technique is presented in this thesis for the determination of these parameters. A monochromatic source experiment is proposed because of the desirability of such an experiment for determining the acoustic properties of an anelastic medium. The input information is the plane wave reflection coefficent as a function of the angle of incidence at a fixed frequency. A nonlinear integral equation relating the variations of these acoustic properties from a known reference value to the plane wave reflection coefficient is derived. This is then linearised using the Born approximation. The region of validity of the Born approximation is derived and based on this the 
optimum angular aperture for the input data is obtained.

The linearised integral equation is a Fredholm integral equation of the first kind. An acceptable stable solution of the integral equation is obtained by imposing a priori constraints on the solution. The inversion method is tested using synthetic data and inversions are carried out for various examples of the attenuation coefficient profile and the sound speed profile. The results obtained with noise free data show good agreement between the true profiles and the reconstructed profiles. The resolution obtainable with the data set is studied using the resolving power theory of Backus and Gilbert and the inversion method is shown to provide adequate resolution. The effect of additive noise in data is examined and inversions performed with noisy data yielded stable acceptable results.

Thesis supervisor: George V. Frisk, Associate Scientist, Woods Hole Oceanographic Institution. 


\section{ACKNOWLEDGEMENTS}

I started my graduate studies with a lot of trepidation but the last five years as a student turned out to be a memorable, enjoyable, and rewarding experience. This was in no small measure due to the environment provided by the MIT/WHOI Joint Program and the support I enjoyed from my family, friends and teachers. I am deeply indebted to my thesis adviser, the inimitable George Frisk, who helped me to see the woods from the trees and devoted hours in discussions on the various aspects of the problem studied in this thesis. I enjoyed this exchange of views and look forward to many years of collaboration in the future.

To my colleagues Ching Sang Chiu, Jim Miller, Andre Merab, Peter Stein, and Mike Wengrovitz, I owe a note of special thanks for the time we spent in fruitful discussions on aspects of ocean acoustics.

Without the support of my wife Hema, my mother and my children this mad dream of mine would never have become a reality. To my dear friend $K$. Narendra, whose faith in me was primarily responsible for my return to the academic arena after over two decades, I owe a sincere thanks.

Finally I acknowledge the financial support provided by the education office in the Woods Hole Oceanographic Institution and the Office of Naval Research. 
$-4-$

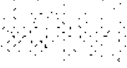




\section{Table of Contents}

\section{List of Figures}

1. Introduction 14

1.1 Background $\quad 14$

1.2 Overview 21

1.3 References $\quad 24$

2. Acoustic Modelling of Marine Sediments 26

2.1 Dispersion and attenuation of compressional waves 26

2.1.1 Visco-elastic model 31

2.1.2 'Biot' model 36

2.1.3 Compressional wave attenuation vs Depth 38

2.1.4 Conclusions $\quad 43$

2.2 Summary of other essential characteristics of the geoacoustic 47 parameters.

2.2.1 Compressional wave speed as a function of depth $\quad 47$

$\begin{array}{ll}\text { 2.2.2 Shear wave speed as a function of depth } & 47\end{array}$

2.2.3 Shear wave attenuation and dispersion $\quad 47$

2.2.4 Shear wave attenuation as a function of depth 48

$\begin{array}{ll}2.2 .5 \text { Density as a function of depth } & 48\end{array}$

2.3 Ocean bottom model 48

2.4 References $\quad 54$

3. Determination of the Attenuation Profile 56

3.1 Review of methods in the literature 58

3.2 Determination of the attenuation profile from the plane-wave 70 reflection coefficient

3.2.1 Determination of plane-wave reflection coefficient(step 1) 70

3.2.2 Determination of sound speed and density profiles(step 2) $\quad 72$

3.2.3 Determination of attenuation coefficient(step 3) 72

3.3 Statement of the problem for the determination of attenuation 73 coefficient

$\begin{array}{ll}3.4 \text { References } & 76\end{array}$

4. The Forward Problem 78

4.1 Uniform asymptotic solution $\quad 82$

4.2 Propagator matrix method $\quad 83$

$\begin{array}{ll}4.3 \text { References } & 93\end{array}$

5. Formulation of the Inverse Problem 94 
$-6-$

5.1 Inverse problem formulation using Born approximation 97

5.1.1 Derivation of integral equation $\quad 97$

5.1.2 Region of validity of the Born approximation 101

5.2 Inverse problem formulation using the Rytor approximation $\quad 104$

5.3 Summary $\quad \cdot 107$

$\begin{array}{ll}\text { 5.4 References } & 108\end{array}$

6. Linear Inverse Theory 108

6.1 Fredholm integral equation of the first kind 110

B.1.1 Non-uniqueness $\quad 110$

6.1.2 Instability 111

6.2 Solution in parameter space - Error free data 115

6.2.1 Generalised loverse $\quad 115$

6.2.2 Regularisation method 118

6.3 Solution in parameter space-Data with errors 122

6.3.1 Generalised Inverse $\quad 122$

6.3.2 Regularisation Method 123

6.3.3 Maximum likelibood estimate $\quad 123$

6.3.4 Minimum variance estimate $\quad 124$

6.4 Solution in function space-Error free data 125

6.4.1 Spectral expansion method $\quad 125$

6.4.2 Method of Backus and Gilbert 127

6.5 Solution in function space- Data with errors 129

6.6 Non-linear problem $\quad 129$

6.7 Summary 130

6.8 References 131

7. Inversion for Acoustic Parameters 133

7.1 Linearisation and iteration procedure $\quad 134$

7.2 Reconstruction of the attenuation profile 138

7.2.1 The regularization scheme 138

7.2.2 Angular aperture for data points 144

7.3 Effect of experimental and geoacoustic parameters 153

$\begin{array}{ll}\text { 7.3.1 Frequency } & 153\end{array}$

7.3.2 Attenuation coefficient $\quad 156$

7.3.3 Depth of sediment 156

7.3.4 Strength of the discontinuity at the interface $\quad 161$

$\begin{array}{ll}7.4 \text { Examples of reconstruction } & 161\end{array}$

7.5 Born and Rytor approximations $\quad 175$

$\begin{array}{ll}7.6 \text { Resolution } & 181\end{array}$

7.6.1 Measure of resolution ]

$\begin{array}{ll}\text { 7.6.2 Variation of aperture size } & 191\end{array}$

7.6.3 Variation of the number of data points 194

7.7 Errors

7.7.1 Errors in the mindel

7.7.2 Errors in data 211

7.8 Summary 219

$\begin{array}{ll}7.9 \text { References } & 220\end{array}$

8. Acoustic Parameters from Eigenvalues 222 
8.1 Derivation of integral equation $\quad 225$

8.2 Inversion for sound speed using synthetic data 230

$\begin{array}{ll}8.3 \text { Inversion of real data } & 237\end{array}$

$\begin{array}{ll}8.4 \text { References } & 239\end{array}$

9. Conclusions $\quad 240$

$\begin{array}{ll}9.1 \text { Suggestions for future work } & 242\end{array}$

9.1.1 Testing the validity with real data $\quad \mathbf{2 4 2}$

$\begin{array}{ll}\text { 9.1.2 Effect of shear } & \mathbf{2 4 3}\end{array}$

$\begin{array}{ll}\text { 9.1.3 Shallow water problem } & \mathbf{2 4 3}\end{array}$

9.1.4 Inversion from the point source pressure field 243

$\begin{array}{ll}\text { Appendix A. } & 244\end{array}$

$\begin{array}{ll}\text { Appendix B. } & \mathbf{2 4 9}\end{array}$ 


\section{List of Figures}

Figure 1-1: Ray diagram for sound waves interacting with the bottom 14

Figure 1-2: Schematic of experiment with impulsive source 15

Figure 1-3: Schematic of experiment with monochromatic source $\quad 16$

Figure 2-1: Variation of the attenuation coefficient with frequency- 27 reproduced from Hamilton $[4]$

Figure 2-2: Kelvin-Voigt model of visco-elastic solid 29

Figure 2-3: Response of Kelvin-Voigt solid to suddenly applied load 29

Figure 2-4: Standard linear solid $\quad 32$

Figure 2-5: Response of standard linear solid to suddenly applied load $\quad 32$

Figure 2-6: Variation of the attenuation coefficient with frequency- 33 linear solid[3]

Figure 2-7: Variation of the attenuation coefficient with frequency 36 using Biot model[13,14]

Figure 2-8: Variation of the attenuation coefficient with frequency - 38 reproduced from McDaniel[15]

Figure 2-9: Variation of the compressional wave speed with frequency- $\quad 39$ reproduced from $\mathrm{McD}$ aniel [15]

Figure 2-10: Variation of the compressional wave attenuation with 40 depth - reporduced from Hamilton[4]

Figure 2-11: Measurements of attenuation coefficient|22] 41

Figure 2-12: Variation of the compressional wave velocity with depth - 43 reproduced from Hamilton[4]

Figure 2-13: Variation of the compressional wave speed with depth in 44 sandy sediments - reproduced from Hamilton[4]

Figure 2-14: Variation of the shear wave speed with depth-reproduced 45 from Hamilton $[4]$

Figure 2-15: Variation of density with depth - reproduced from 48 Hamilton[4]

Figure 2-18: Seismic profiling of the ocean bottom 49

Figure 2-17: Ocean bottom model 50

Figure 3-1: Configuration of experiment by Anderson and Blackman[7] 56

Figure 3-2: Configuration of experiment by Jacobson etal[8] 58

Figure 3-3: Configuration of experiment by Stoll[11] 61

Figure 3-4: Configuration of experiment by Mitchell and Focke[12] $\quad 63$

Figure 3-5: Configuration of experiment by Frisk etal[6] 68

Figure 3-6: Steps for obtaining geoacoustic parameters from pressure 70

field

Figure 3-7: Exact and guess model for the ocean bottom

Figure 4-1: Oceari bottom model 78

Figure 4-2: Single layer model

Figure 4-3: Multi-layer model

Figure 5-1: Ocean bottom model $\quad 97$

$\begin{array}{ll}\text { Figure 6-1: } & 118\end{array}$

Figure 7-1: Simplified ocean bottom model to study behaviour of 144 Green's function 
Figure 7-2: Magnitude of Rayleigh reflection coefficient st the two 145 interfaces

Figure 7-3: Magnitude of Green's function as function of borizontal 147 waresumber, Frequency $=200 \mathrm{~Hz}$

Figure 7-4: Magnitude of Green's function as function of horizontal .I50 wavenumber, Frequency $=50.0 \mathrm{~Hz}$

Figure 7-5: Magnitude of Green's function as sunction of horizontal 151 wavenumber, Frequency $=100.0 \mathrm{~Hz}$

Figure 7-6: Reconstruction of constant attenuation of $0.02 \mathrm{~dB} / \mathrm{m}$ in the 153 layer, Frequency $=100 \mathrm{~Hz}$

Figure 7-7: Reconstruction of constant attenuation of $0.02 \mathrm{db} / \mathrm{m}$ in the 154 layer, Frequency $=25 \mathrm{~Hz}$

Figure 7-8: Reconstruction of constant attenuation of $0.005 \mathrm{~dB} / \mathrm{m}$ in 156 the layer, Frequency $=25 \mathrm{~Hz}$

Figure 7-0: Magnitude of Green's fubction as a function of horizontal 157 wavenumber, Depth of sediment $=40 \mathrm{~m}$

Figure 7-10: Magnitude of Green's function as a function of horizontal 158 W'avenumber, Depth of sediment $=120 \mathrm{~m}$

Figure 7-11: Reconstruction of constant attenuation in the layer, layer 159 thickness $=160 \mathrm{~m}$, frequency $=25 \mathrm{~Hz}$

Figure 7-12: Magnitude of Green's function as function of borizontal 161 wavenumber, Sound speed in sub-bottom $=2500 \mathrm{~m} / \mathrm{sec}$

Figure 7-13: Magnitude of Green's function as a function of horizontal 162 wavenumber, Sound speed in sub-bottom $=3000 \mathrm{~m} / \mathrm{sec}$

Figure 7-14: Attenuation coefficient profile shapes

Figure 7-15: Reconstruction of profile in figure 7-14(a)

Figure 7-16: Reconstruction of profile in figure 7-14(b)

Figure 7-17: Reconstruction of profile in figure 7-14(c)

Figure 7-18: Reconstruction of profile in figure 7-14(d)

Figure 7-18: Reconstruction of profile in figure 7-14(e)

Figure 7-20: Reconstruction of profile in figure 7-14(e)

Figure 7-21: Realistic ocean model

Figure 7-22: Reconstruction of profile for realistic ocean model

Figure 7-23: Reconstruction of profile in figure 7-14(a) - Born 175 approximation

Figure 7-24: (Reconstruction of profile in figure 7-14(a) - Rytov 176 approximation

Figure 7-25: Reconstruction of profile in figure 7-14(b) - Born 177 approximation

Figure 7-28: Reconstruction of profile in figure 7-14(b) Rytor 178 approximation

Figure 7-27: Reconstruction of profile in figure 7-14(a) - Rytor 178 approximation

Figure 7-28: Resolving kernel for profile $7-14(a)$

Figure 7-28: Resolving keruel for profile $7-14$ (t)

185

$18:$

Figure 7-30: Resolving leruel for profile i-14(c)

18 :

Figure 7-31: Fesolving kerdel for for int: $7-14(d)$

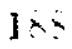

Figure 7-32: Oceas bottom model

Figure 7-33: Reconstruction for lossless model using Gelfand-Levitan

184 method

Figure 7-34: Reconstruction of losymodel using. Gelfand-Leritar los metbod 
$-10-$

Figure 7-35: Effect of error in sound speed on reconstruction 198

Figure 7-36: Effect of error in density on reconstruction 199

Figure 7-37: Simultaneous reconstruction - sound speed profile; case (i) 206

Figure 7-38: Simultaneous reconstruction - Attenuation profile; case(i) 207

Figure 7-39: Simultaneous reconstruction - Sound speed profile; case(ii) 208

Figure 7-40: Simultaneous reconstruction - Attenuation profile; case(ii) 209

Figure 7-41: Reconstruction of profile in figure 7-14(a) with noisy 211 data-40dB

Figure 7-42: Reconstruction of profile in figure 7-14(b) with noisy data 212 $-40 \mathrm{~dB}$

Figure 7-43: Reconstruction of profile in figure 7-14(c) with noisy data- 213 $40 \mathrm{~dB}$

Figure 7-44: Reconstruction of profile in figure 7-14(a) with noisy data 214 $50 \mathrm{~dB}$

Figure 7-45: Reconstruction of profile in figure 7-14(b) with noisy data 215 $50 \mathrm{~dB}$

Figure 7-46: Reconstruction of profile in figure 7-14(c) with noisy data 216 $50 \mathrm{~dB}$

Figure 8-1: Experimental configuration for shallow water experiment 222

Figure 8-2: Hankel transform of measured pressure field 223

Figure 8-3: Ocean model 225

Figure 8-4: Exact and guess model - case(i) 230

Figure 8-5: Exact and guess model - case(ii) 231

Figure 8-6: Reconstructed profile - case(i) 232

Figure 8-7: Reconstructed profile - case(ii) 233

Figure 8-8: Reconstructed profile using data from all three frequencies- $\mathbf{2 3 4}$ case(i)

Figure 8-9: Reconstructed profile using data from all three frequencies- 235 case(ii)

Figure 8-10: Inversion of real data 


\section{List of Tables}

Table 5-I: Real and imaginary parts of the wavenumber at $220 \mathrm{~Hz}$ for 94 marine sediments

Table 7-I: Parameters used for ocean bottom model

Table 7-II: Resolution lengths for the different examples of 189 reconstruction

Table 7-III: Resolution length and aperture size

Table 7-IV: Resolution length and number of data points

Table 7-V: Exact and erroneous sound speed profile

Table 7-VI: True and background model parameters - case(i)

201

Table 7-VII: True and background model parameters - case(ii) 


\section{Chapter 1 Introduction}

The prediction of sound propagation in the ocean requires a knowledge of the acoustic properties of marine sediments. In the range of frequencies of interest ( 50 - $500 \mathrm{~Hz}$ ) marine sediments can be modelled as a fluid[1]. Therefore, the acoustic properties of marine sediments of interest are the compressional wave speed, the compressional wave attenuation and the density. Further when the assumption of horizontal stratification is made, these parameters are a function of depth only. In this thesis, we present an inverse method based on a perturbation technique for obtaining these parameters. The input information is the plane wave reflection coefficient for the bottom as a function of angle of incidence at a fixed frequency.

\subsection{Background}

Figure 1-1 is a typical ray diagram for low frequency sound $(50-500 \mathrm{~Hz})$ interacting with the bottom. The rays emanate from the source and are partially reflected at the water/ocean bottom interface and the energy entering the bottom, therefore, depends on the impedance contrast at this interface. The rays that penetrate into the bottom are,however, refracted back into the water column because of the increasing sound speed. As the rays travel in this region, some of the energy associated with them is converted into heat. The attenuation coefficient is a measure of the amount of energy dissipated in this manner. Thus the compressional wave speed, the compressional wave attenuation and the density of the sediment 


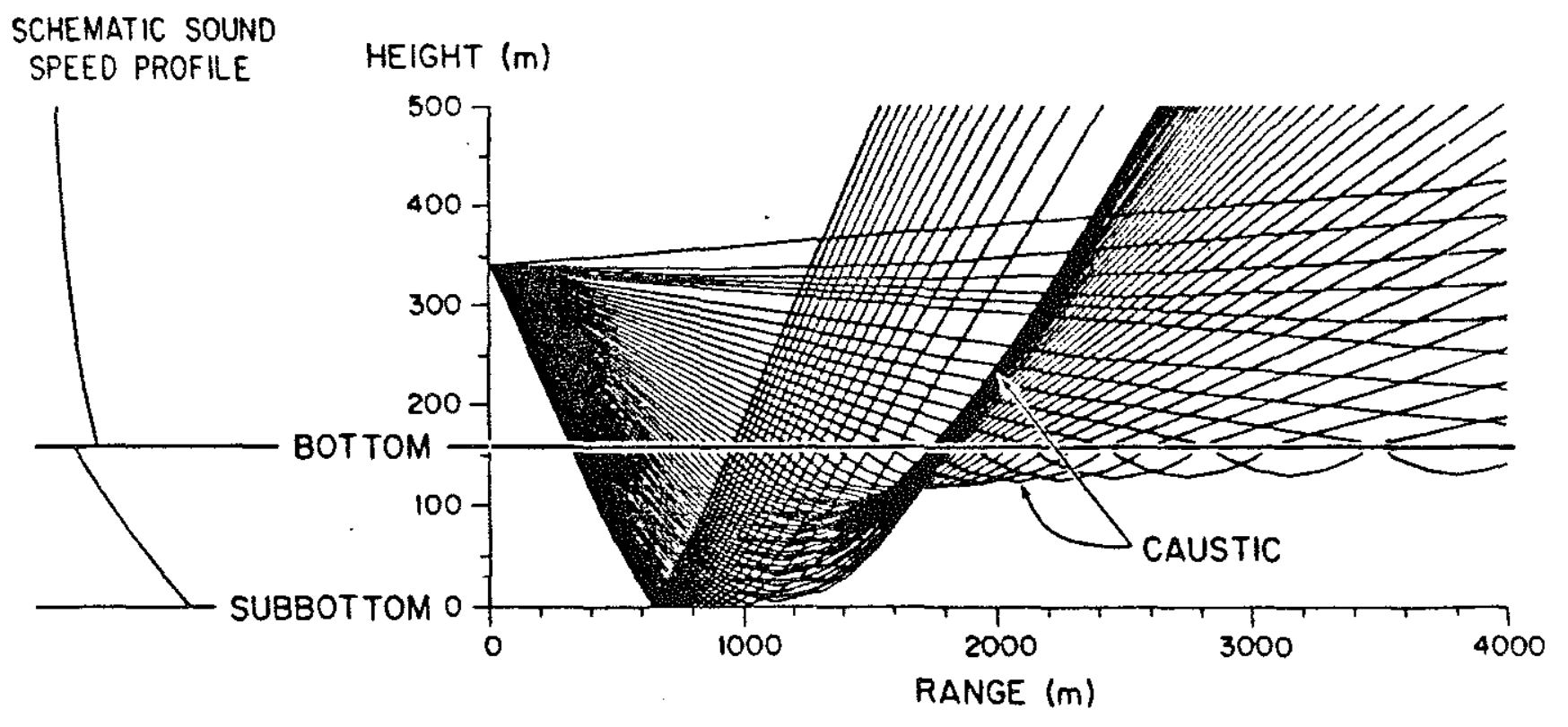

Figure 1-1: Ray diagram for sound waves interacting with the bottorn 
$-14-$

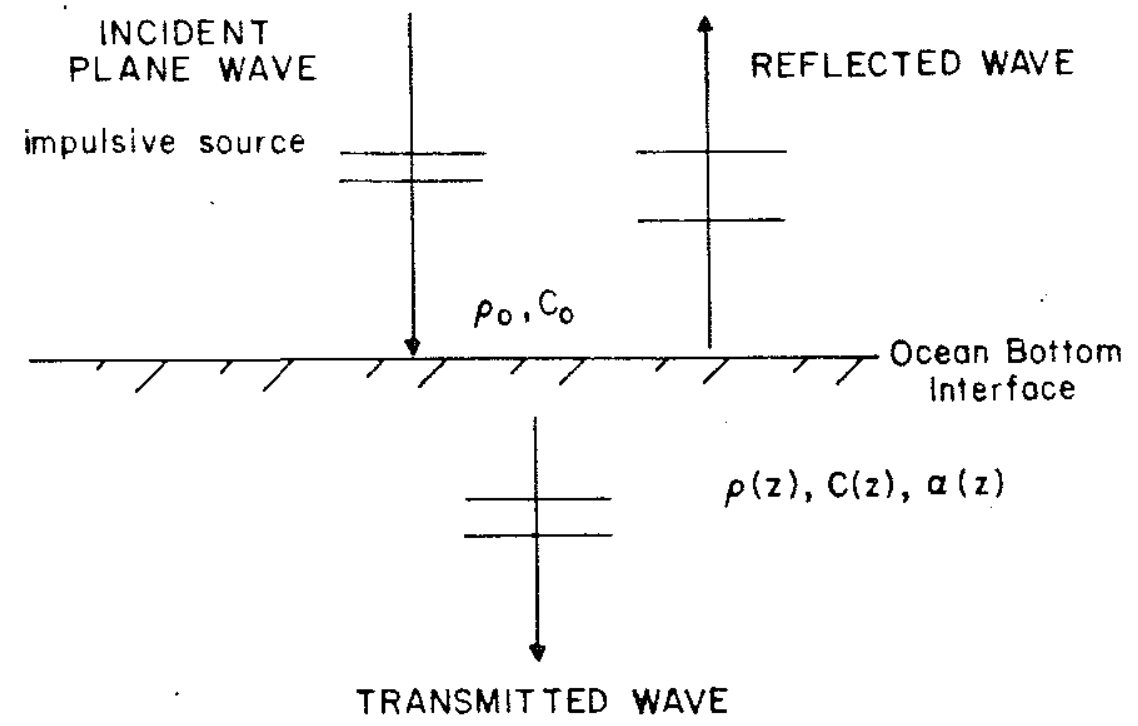

Figure 1-2: Schematic of experiment with impulsive source 


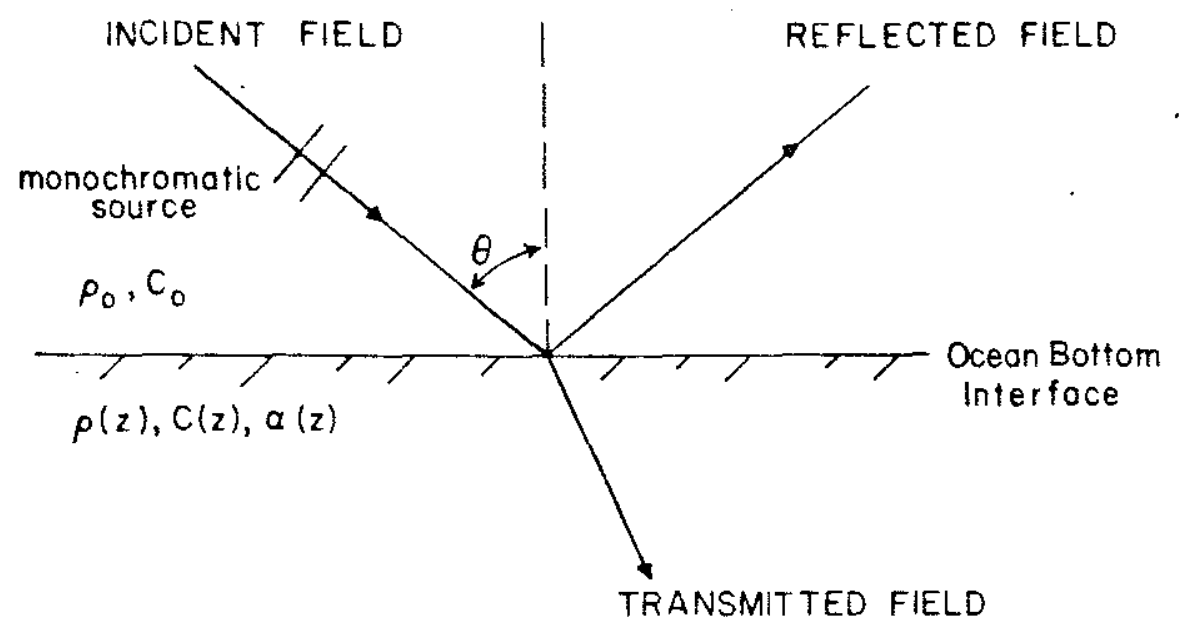

Figure 1-3: Schematic of experinent with monochromatic source 
have a direct effect on the propagation of sound in the ocean.

The forward scattering problem deals with the determination of the wave field scattered from an object with known properties for a given incident wave field. In contrast to this, in the inverse scattering problem the scattered field for a given incident field is measured at the boundary of the object or at points external to the object and from this the properties of the object are inferred. The determination of the acoustic properties of the ocean bottom from the plane wave reflection coefficient falls into this category.

Two approaches are found in the literature for determining the acoustic properties of a layered medium from its reflection response. These are exact methods and approximate methods. A review of exact inversion methods using reflection coefficient data for layered media is given by Newton[2]. Our approach is, however, based on an approximate method developed by Cohen and Bleistein[3]. In the setting of geophysical inverse problems, their method was applied to the determination of the compressional wave speed in the interior of the earth. A schematic diagram for the experiment is shown in Figure 1-2. The source is an impulsive plane wave source. The backscatterd field at the location of the source is measured. Using perturbation theory, the back scattered field is related to the variation of the compressional wave speed from a known reference value and a linear integral equation obtained. The linearising approximation is called the Born approximation. When the reference wave speed is a constant a closed form solution to the integral equation is obtained. In the two dimensional case[4], the experiment consists of using a line of sources which is equivalent to repeating the experiment for the one dimensional problem at a number of locations along a line. In this case the variation of the compressional wave speed from its reference is in two spatial coordinates. This is related to the backscattered field measured along the line 
through a linear integral equation as before.

Subsequent to the publication of the first paper by Cohen and Bleistein, a number of papers have appeared in the field of seismology which are based on a Born type approximation[5-9]. Features common to all these are as follows.

1. The response to an impulsive source at the surface of the medium being probed is measured at the same location as the source or at a fixed off-set from it. This is equivalent to measuring the plane wave reflection coefficient as a function of frequency at normal incidence or at a fixed angle of incidence.

2. The reference wave speed and density are generally assumed to be constants. Raz[10],Clayton and Stolt[11], and Bleistein and Gray[12], however, also consider the case when the reference compressional wave speed is depth dependent.

3. The medium is lossless.

4. The variation of true wave speed from the reference speed is small and the Born approximation is valid at all frequencies of the broad band source.

The experiment that we propose uses a CW source and the input data in this case is the plane wave reflection coefficient at various angles of incidence. A schematic of the experiment is shown in Figure 1-3. Exact methods for obtaining the acoustic parameters of the medium from the angular dependence of the reflection coefficient have been proposed by Hooshayar and Razavy[13] and Stickler[14]. Schaubert and Mittra[15] and Roger[16] present inverse methods based on perturbation techniques for determining the permittivity of a lossless dielectric medium using a monochromatic source to probe the medium. However, a perturbation method using the angular dependence of the plane wave reflection coefficient as input information has not been applied to the determination of all three acoustic parameters of the ocean bottom. The features of this method that 
distinguish it from otber Born approximation methods are

1. The input information is the plane wave reflection coefficient as a * function of the angle of incidence at 8 fixed frequency.

2. The reference wave speed, density and the wave attenuation can vary arbitrarily with depth.

3. The medium can be lossy.

4. The method yields all three acoustic parameters.

5. The Born approximation is not uniformly valid over all the angles and therefore band limited information is used.

The linear integral equation obtained by this method is a Fredholm integral equation of the first kind. These equations suffer from problems of non-uniqueness and instability of the solution. We obtain a stable acceptable solution by using the regularisation method due to Phillips[17] and Twomey[18].

Though the problem as described above involves the determination of all three acoustic parameters, the starting point of this thesis was the development of a method for obtaining the compressional wave attenuation for the ocean bottom. In studying a possible method, we noted that it required an exact knowledge of the sound speed and density profiles for the bottom. We found that even small errors in these two parameters adversely affected the determination of the attenuarion coefficient. This led to the present approach where instead of trying to correct for the errors in the sound speed and density, these are treated as unknowns in a general formulation that yields corrections to the sound speed and density frofites togetber with the attenuation coeficient profile.

We will, therefore, develop the method for the determination of the attenuation coefficient profile for the bottom and then extend it to deal with 
perturbations in the compressional wave speed and density. We do this not only because it is the path that our investigation took us but also because the need to determine the attenuation coefficient and the problems in its determination will then be brought into focus.

When the ocean is a shallow water wave guide, we use eigenvalues of the modes trapped in the water column as input information for the inverse method. A linear Fredholm integral equation of the first kind relating the variations in the acoustic parameters from a known reference value to the modal eigenvalues is obtained. This equation can then be solved employing any one of the methods available for solving this class of integral equations.

\subsection{Overview}

In Chapter 2 we study the propagation of plane waves in an anelastic medium to obtain a correct model for the sediments. This is important as it can influence the type of experiment performed for obtaining the acoustic parameters.

In Chapter 3, after a brief review of the methods described in the literature for the determination of the attenuation profile in marine sediments, we propose a method where, as a first step towards inferring the acoustic properties of the ocean bottom, we obtain the plane wave reflection coefficient for the bottom as function of angle of incidence[19]. A sequence of steps which can then be used to obtain all the acoustic parameters of the bottom is indicated.

In Chapter 4 we describe methods for solving the forward problem i.e finding the acoustic field in the bottom given its acoustic parameters. A numerically stable propagator matrix algorithm is presented. 
We then formulate the inverse problem for the determination of the attenuation coefficient profile in the bottom. This is done in Chapter 5 . Two approaches to linearisation, namely the Born and Rytov methods, are used and in each case an integral equation relating the attenuation coefficient profile to the reflection coefficient is derived.

Chapter 6 is a predominantly review chapter, where we study the problems of instability and non-uniqueness encountered in solving inverse problems of this type. Various methods described in the literature for solving these problems are then described with a view to bringing out the underlying commonality of all the approaches. The method of solving nonlinear problems by linearisation is then described.

In Chapter 7 we continue with the solution of the inverse problem formulated earlier. Adopting a regularisation scheme for overcoming problems of nonuniqueness and instability several examples of inversion are performed using synthetic data. The sensitivity of the reconstruction to various parameters and other related issues are examined. We extend the formulation of the inverse problem to determine perturbations in other acoustic parameters and demonstrate this by simultaneously solving for the attenuation profile and perturbations in the sound speed profile. We also show how the procedure described by Backus and Gilbert[20] for determining the resolvability of a finite data set can be applied to situations when all the quantities are complex. The effect of noisy data on reconstruction is also investigated.

We then show that in the shallow water context, the problem can be reformulated to relate the perturbations in the acoustic parameters to the changes in the eigenvalues. This is done in Chapter 8 . Some preliminary results of inversion using synthetic and real data are presented. 
Chapter 9 is the concluding chapter where we indicate the directions for future work in this field. 


\subsection{References}

1. G. Fryer, "Reflectivity of ocean Lottom al low frequency," J. Acoust. Soc. Am., 63(11), 35-43 (1078).

2. R.G. Newton, "Inversion of reflection data for layered media: a review of exact methods," Geophys. J. R. Astr. Soc., 65, 191-215 (1981).

3. J.K. Cohen, and N. Bleistein, "An inverse method for determining small variations in propogation speed," SIAM J. Appl. Math., 32(4), 784-798 (1877).

4. J.K. Cohen, and N. Bleistein, "Velocity inversion procedure for 8coustic waves," Geophysics, 44(6), 1077-1085 (1979).

5. S.H. Gray, J.K. Cohen, and N. Bleistein, "Velocity inversion in a stratified medium with separated source and receiver," J. Acoust. Soc. Am., 68, 24-240 (1880).

6. S.H. Gray, and F.G. Hagin, "Towards precise solution of one dimensional inverse problems," SLAM J. Appl. Math., 42, 346-352 (1882).

7. S.Raz, "Three dimensional velocity profilr inversion from finite off-set scattering data," Geophysics, 46(6), 837-842 (1981).

8. S. Raz, "Direct reconstruction of velocity and density profiles from scattered field data," Geophysics, 46(6), 832-836 (1881).

9. S. Raz, "An algorithm for profile reconstruction in the presence of a known background slab," Radio Science, 15(3), 627-636 (1880).

10. S. Raz, "A direct profile inversion: beyond the Born model," Radio Science, 16, 347-353 (1981).

11. R.W. Clayton, and R.H. Stolt, "A Born UKBJ inversion method for acoustic reflected data," Geophysics, 46, 1558-1568 (1981).

12. N. Bleistein, and S.H. Gray, "An extension of the Born inversion method to depth dependent reference profile," Technical Report No. CWP-019. Center for Wave Phenomena, Colorado School of Mines. Colorado (19:4). 
13. M.A. Hooshayar, and M. Razavy, "A method for constructing wave velocity and density profiles from the angular dependence of the reflection coefficent,” J. Acoust. Soc. Am., 73(1), 19-23 (1983).

14. D.C. Stickler, "Inverse scattering in a stratified medium," J. Acoust. Soc. Am., 74(3), 994-1005 (1983).

15. D.H. Schaubert, and R. Mittra, "A spectral domain method for remotely probing stratified media," IEEE Transactions on Antennas and Propagation, AP-25(2), 261-265 (1977).

16. A. Roger, "Determination of the index profile of a dielectric plate from scattering data," in Applied Inverse Problems, (Springer, New York, 1978).

17. D.L. Phillips, "A technique for numerical solution of Fredholm integral equation of the first kind," J. Assoc. Compt. Mach., 9, 84-97 (1962).

18. S. Twomey, "On the numerical solution of Fredholm integral equation of the first kind by inversion of the linear system produced by quadrature," J. Assoc. Compt. Mach., 10, 97-101 (1963).

19. G.V. Frisk, D.R. Mook, J.A. Doutt, E.E. Hays, and A.V. Oppenheim, "The application to real data of a technique for measuring the plane wave reflection coefficient of the ocean bottm, "J. Acoust. Soc. Am., 72(s1)S97(a) (1982).

20. G. Backus and F. Gilbert, "The resolving power thery of gross earth data," Geophys. J. R. Astr. Soc., 16, 169-205, (1968). 


\section{Chapter 2 \\ Acoustic Modelling of Marine Sediments}

Acoustic transmission in the ocean at low frequency is dependent on the geoacoustic properties of the seabed, namely, the speed and attenuation of the compressional and shear waves and density. A complete specification of the seabed will contain the wave speeds and attenuations as a function of the three spatial coordinates and frequency, and density as a function of the spatial co-ordinates.

In this chapter we develop a model for the ocean bottom that will be used in the analysis to be presented in the following chapters of this work. In this context, we study the frequency dependence of attenuation and the dispersion of the wave speeds due to attenuation. These are important considerations in the design of a suitable experiment for the measurement of the attenuation coefficient. We also summarise the other geoacoustic characteristics associated with wave propagation in marine sediments.

\subsection{Dispersion and attenuation of compressional waves}

We start by studying the propagation of plane compressional waves in an attenuating medium. In deriving the classical loss-less wave equation it is assumed that the material behaves in an elastic manner. The result of such an assumption is that sound waves propagate in the medium without change of shape and suffer no attenuation. However, in real sediments the waves decay as they propagate. Their energy is gradually converted to heat. This process is the result of a variety of 
$-25-$

mechanisms which operate for most part at the microscopic level. At the macroscopic level the energy loss is given such terms as internal friction, attenuation and anelasticity.

The most direct method of defining 'internal friction' is as the ratio $-(\Delta E) / E$ where $\Delta E$ is the energy lost in each cycle because the material is not perfectly elastic and $E$ is the peak energy stored in the volume of material taken through the stress cycle at a frequency $\omega$. The specific attenuation coefficient $Q$ is defined as

$$
\frac{2 \pi}{Q}=-\frac{\Delta E}{E}
$$

This factor $Q$ can be measured without any assumption being made on the attenuating mechanisms and is therefore used to describe attenuating behaviour of the medium in a phenomenological sense.

For a plane propagating wave, the effect of 'internal friction' will be the gradual decay of the amplitude of the wave as it propagates. Since the energy is propotional to the square of the amplitude,

$$
\frac{1}{Q(\omega)}=-\frac{\Delta E}{2 \pi E}=-\frac{\Delta A}{\pi A}
$$

In the above expression $\Delta A$ is the change in amplitude over one cycle or $\Delta A=(d A / d x) \lambda$ where $\lambda$ is the wave length.

$$
\begin{aligned}
& \Delta A=\frac{d A}{d x} \frac{2 \pi C(\omega)}{\omega} \\
& A(x)=A_{0} \exp \left(-\frac{\omega x}{2 C(\omega) Q(\omega)}\right)
\end{aligned}
$$

Therefore, for a propagating wave, 


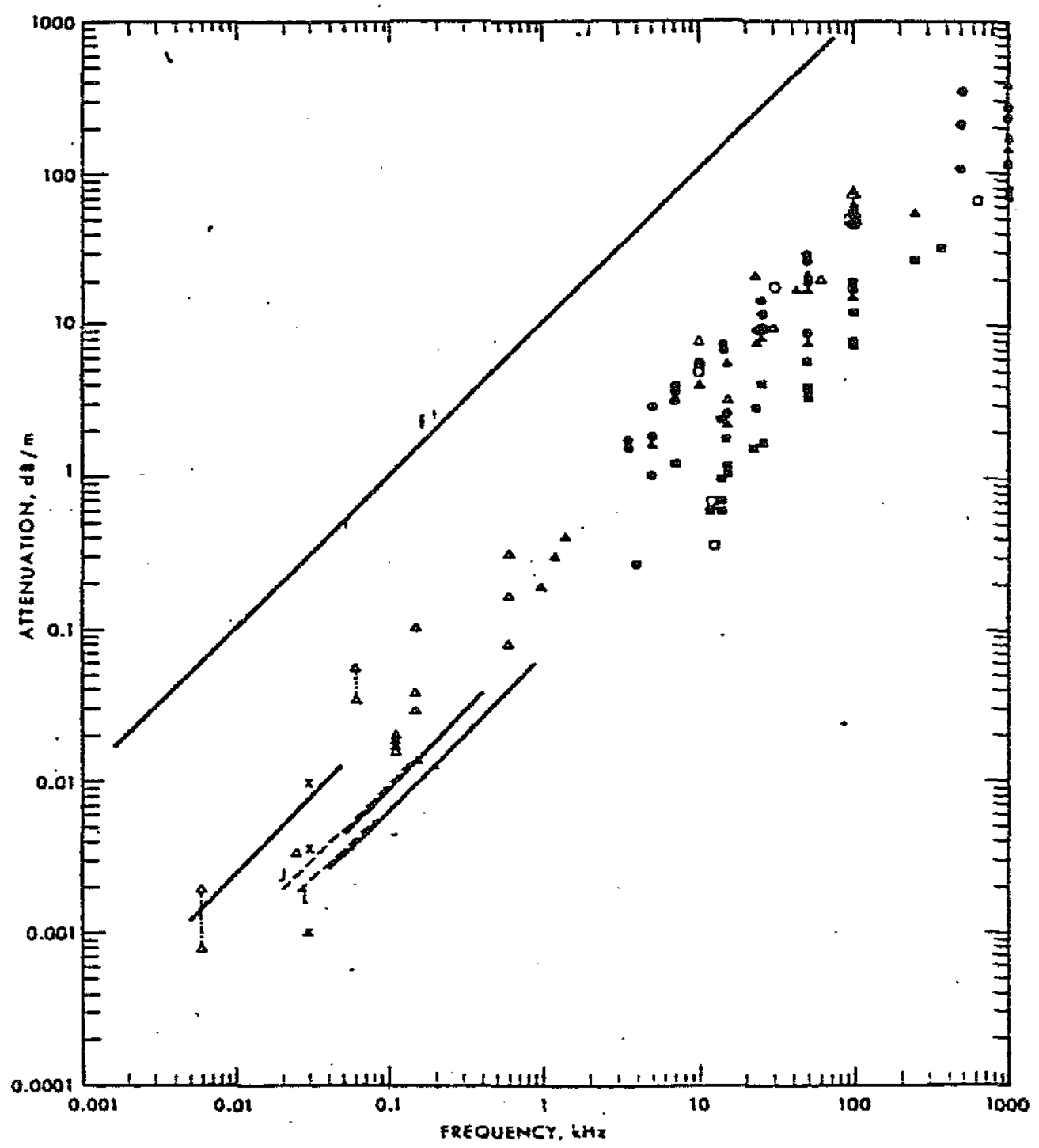

Figure 2-1: Variation of the attenuation coefficient with frequency-reproduced from Hamilton[4] 


$$
\begin{gathered}
A(x, t)=A_{0} \exp \left[i\left(\frac{\omega}{C(\omega)}+i \frac{\omega}{Q(\omega) 2 C(\omega)}-\omega t\right)\right] \\
=A_{0} \exp [i(k(\omega)-\omega t]
\end{gathered}
$$

Here $k(\omega)$ is a complex quantity, its imaginary part representing attenuation. Another measure of attenuation found in the literature is the attenuation coefficient which is given by the following expression.

$$
\alpha(\omega)=\frac{\omega}{2 C(\omega) Q(\omega)}
$$

Attenuation in a medium can therefore be accounted for by making the wave number complex.

The attenuation coefficient is a function of frequency by definition. Aki and Richards[1] show that the behaviour of waves propagating in an attenuating medium cannot be explained unless the assumption of dispersion of these waves is made. Therefore both $Q$ and $C$ are functions of frequency. Figure 2-1 is a plot of the attenuation coefficient for compressional waves with respect to frequency based on laboratory/field experiments. Most of the information is in the high frequency region. The experimental results show a rough linear dependence of the attenuation coefficient on frequency in the high frequency range.

Efforts to explain this experimentally observed behaviour have been made by modifying the equations of th classical elastic theory. Different approaches have been proposed. For example Hamilton[4] proposes a visco-elastic model for water saturated sediments while another approach, called the Biot model, which treats the sediment as a porous elastic solid saturated by a viscous fluid has been pursued by Stoll[13]. The two models will be described briefly in the following paragraphs. 


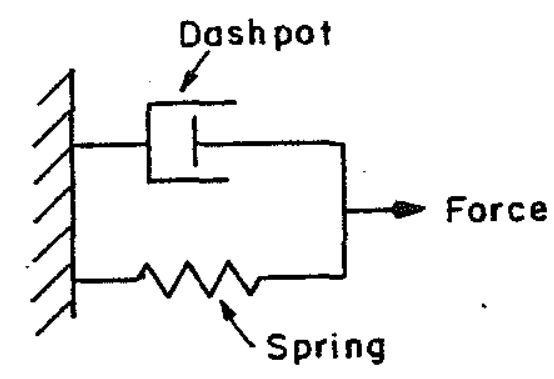

Figure 2-2: Kelvin-Voigt model of visco-elastic solid

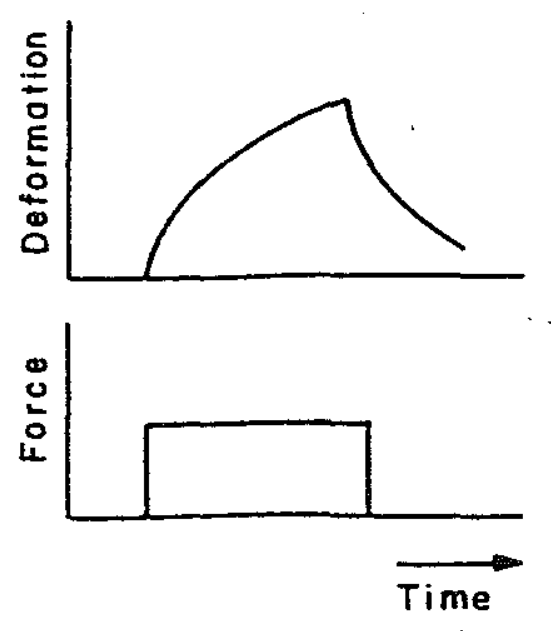

Figure 2-3: Response of Kelvin-Voigt solid to suddenly applied load 


\subsubsection{Visco-elastic model}

In the classical elastic theory, the stress and the strain are related by a linear relationship such as

$$
\sigma \pm B \epsilon
$$

where $\sigma$ is the stress, $\epsilon$ the strain and $B$ is the constant of propotionality called the elastic constant. In the visco-elastic model the anelasticity is incorporated by modifying the elastic equation to express the stress as a function of strain and strain rate as indicated in equation $(2.8)$, where $d \epsilon / d t$ is the strain rate.

$$
\sigma=B \epsilon+G \frac{d \epsilon}{d t}
$$

We now have two elastic constants B and G. A mechanical system representing this stress strain relationship is shown in Figure 2-2 The spring represents a perfectly elastic body for which the stress-strain relationship is governed by the law

$$
\sigma=B \epsilon
$$

The dashpot represents a perfectly viscous body for which the stress strain relationship is given below.

$$
\sigma=\frac{d \epsilon}{d t}
$$

For a system in which these two elements are in parallel the relationship in equation (2.8) is obtained. The analogies of various other visco-elastic models to mechanical systems are given in Reference 5 .

The model in Figure 2-2 is called the Kelvin-Voigt model and Hamilton[2,4] 
has proposed this model to describe the anelastic behaviour of marine sediments. Kolsky[3] has shown that the elastic equation (2.8) when applied to solids resulted in the Lame's constants $\lambda$ and $\mu$ being replaced by $\lambda+\lambda^{\prime}$ and $\mu+\mu^{\prime}$ where $\lambda^{\prime}$ and $\mu^{\prime}$ govern the energy damping and $\lambda$ and $\mu$ together with the density govern the velocity. Based on this analysis, the following equations for specific attenuation coefficient and compressional wave speed were obtained by Hamilton $[2,4]$.

$$
\begin{aligned}
& \frac{1}{Q(f)}=\frac{\alpha(f) c(f)}{\pi f\left(1-r^{2}\right)} \\
& (\lambda+2 \mu)=\rho c_{p}^{2} \frac{\left(1-r^{2}\right)}{\left(1+r^{2}\right)^{2}}
\end{aligned}
$$

where $r=\alpha(f) C(f) / 2 \pi f$. When damping is small $\mathbf{r}$ is neglected and we recover the equation (2.6). Again when $\mathbf{r}$ is negligible there is no dispersion in the medium. For this visco-elastic model the attenuation coefficient is proportional to the square of frequency[3].

The Kelvin-Voigt model does not exhibit the behaviour of real materials when a load is applied to it. The response of the Kelvin-Voigt model to a suddenly applied stress is shown in Figure 2-3. The strain is zero at $t=0$ and reaches its final value asymptotically with time. However, real materials show instantaneous strain. A model which overcomes this shortcoming is the "standard linear model". In this model the stress and stress rate are expressed as a function of strain and strain rate and is given by equation (2.11)

$$
\sigma+\tau_{\sigma} \frac{d \sigma}{d t}=M_{R}\left(\epsilon+\tau \frac{d \epsilon}{\epsilon t}\right)
$$

where $M_{R}$ is called the deformation modulus, $\tau_{\epsilon}$ is the stress relaxation time under constant strain and $\tau_{\sigma}$ is the strain relaxation time under constant stress. We now 
$-31-$

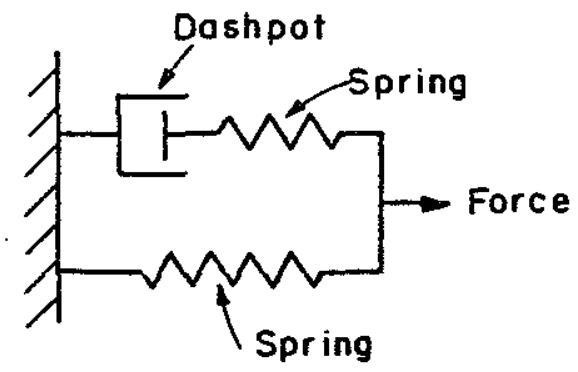

Figure 2-4: Standard linear solid

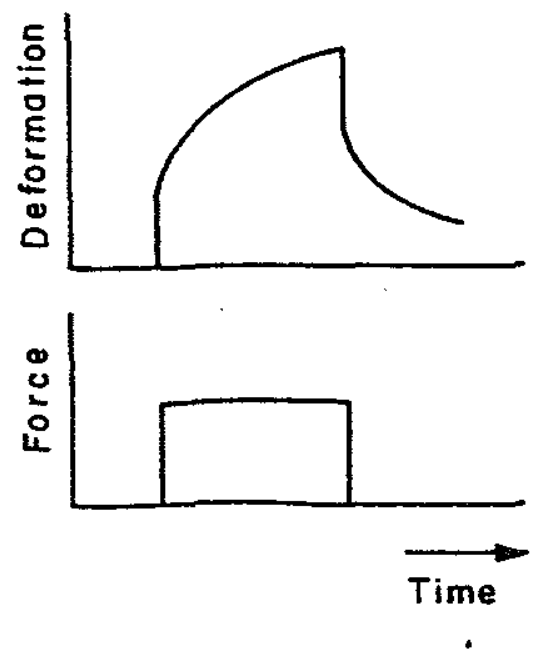

Figure 2-5: Response of standard linear solid to suddenly applied load 


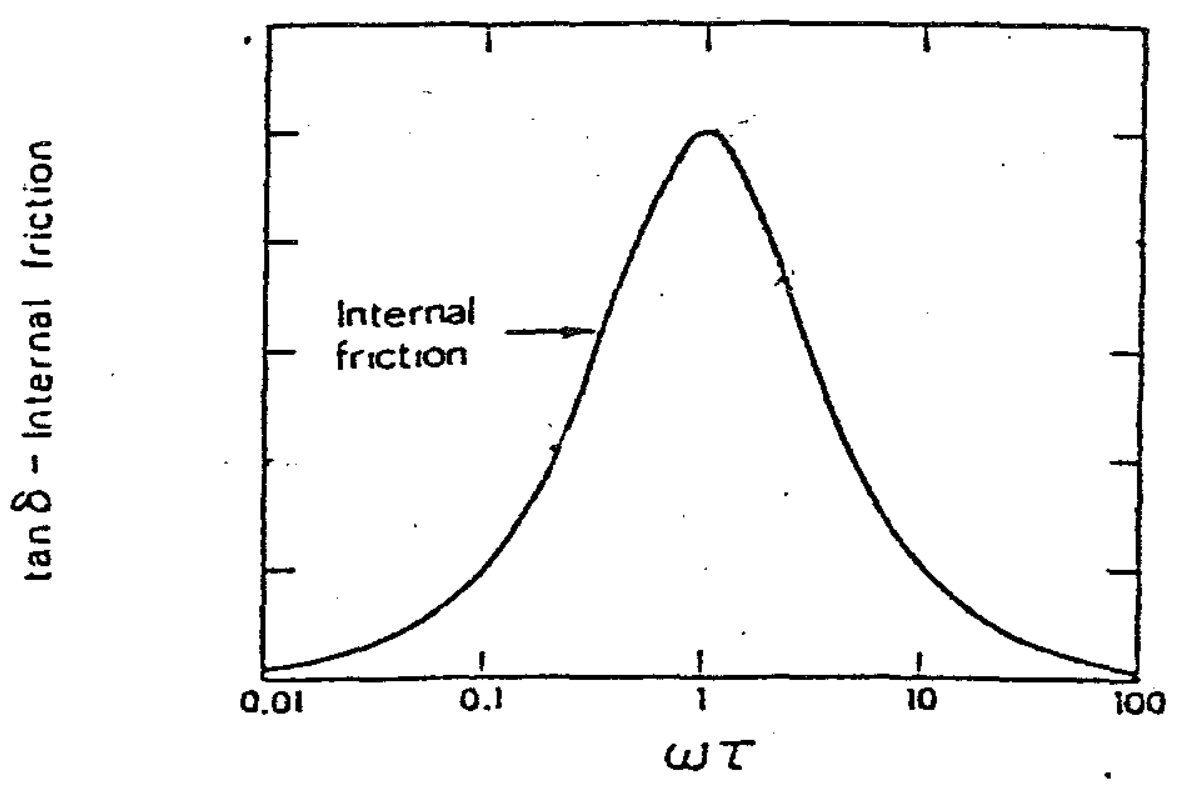

Figure 2-6: Variation of the attenuation coefficient with frequency- linear solid[3]

$$
\begin{aligned}
\tan \delta & =1 / \mathrm{Q}, \omega=2 \pi \mathrm{f} \\
\tau & =\left(\tau_{\epsilon} \tau_{\sigma}\right)^{1 / 2}
\end{aligned}
$$


have three elastic constants. A mechanical system that models this behaviour and its response to a suddenly applied load are in Figures $2-4$ and 2-5.It is seen from Figure 2-5 that there is an instantaneous response of this system to suddenly applied load as is the case for real materials. The dependece of the specific attenuation coefficient for this model is shown in Figure 2-6. The Kelvin- Voigt model and the standard linear model, therefore, cannot explain the linear relationship found experimentally.

However,since energy damping is due to a large number of mechanisms, Liu etal[6] proposed that by considering a large number of relatively closely spaced relaxation mechanisms representing the various damping processes, with each mechanism behaving like a linear solid, the attenuation coefficient can be shown to be linearly related to frequency for earth like materials.

Using the theory of superposition of relaxation mechanisms Liu etal[ 6$]$ derived the following expression for the dispersion of waves.

$$
\frac{C_{p}\left(\omega_{1}\right)}{C_{p}\left(\omega_{2}\right)}=1+\frac{1}{\pi Q} \ln \frac{\omega_{2}}{\omega_{1}}
$$

A similar equation for dispersion has been derived by other investigators [8,9] using entirely different approaches.

The theory of superposition of relaxation mechanisms, therefore, gives the experimentally observed linear dependence of attenuation with frequency and also provides a.dispersion relationship which has been obtained from two completely different approaches. This theory can, therefore, be considered as providing the physical basis for understanding the propagation of waves in an anelastic medium like the earth. Treating consolidated marine sediments as earth-like materials and applying the above theory, the attenuation coefficient can be predicted to have a 
linear relationship with respect to frequency over the entire frequency range and the values of the attenuation coefficient obtained at high frequency can then be extrapolated into the low frequency region. This procedure has been recommended by Hamilton[4]. Experimental results obtained by Frisk[18], Mitchell and Focke[19] and Stoll[20] for attenuation in marine sediments at low frequencies, however, yield values much less than what is predicted by extrapolating the linear law. This casts doubt on the applicability of the superposition theory to marine sediments.

\subsection{2' Biot' model}

Biot $[10,11,12]$ studied the propagation of elastic waves in a system composed of a a porous elastic solid saturated by a viscous fluid. The fluid is assumed to be compressible and may move relative to the frame. In this model, the losses are grouped into two fundementally different categories, one which accounts for the anelasticity of the skeletal frame and the other for the viscosity of the fluid moving relative to the frame.

The theory proposed by Biot was later applied by Stoll[13] and Stoll and Bryan[14] for propagation in ocean sediments. Using this theory, the variation of compressional wave speed with frequency and the variation of attenuation with frequency can be obtained for known sediment properties.

The way in which attenuation varies with frequency depends on the dominance of one or the other of the two modes of energy dissipation that are built into the model, namely, the viscous losses in the fluid as it moves relative to the frame or the frictional losses in the skeletal frame. Figure 2-7 shows two kinds of response predicted by the model. For very fine materials of low permeability, such as silty clay, the losses in the skeletal frame dominate and the authors propose a 


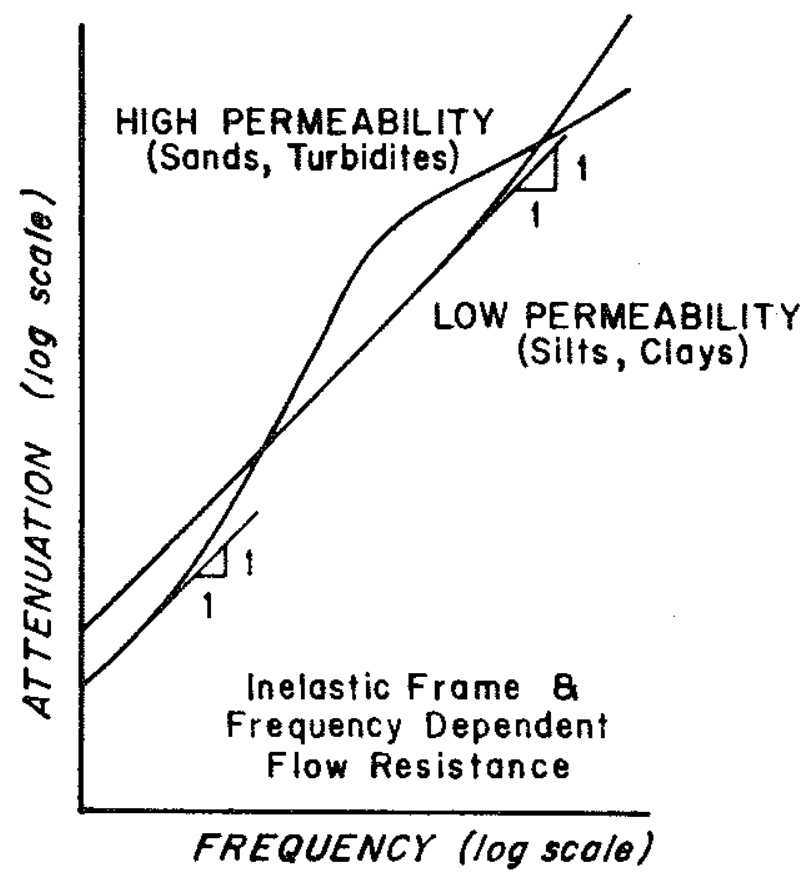

Figure 2-7: Variation of the attenuation coefficient with frequency using Biot model[13,14] 
linear relationship while for high permeability material like coarse sand etc. a dependence $\boldsymbol{f}$ with $\mathrm{n}=2$ at the low frequency end and $\mathrm{n}=1 / 2$ at the high frequncy has been proposed. McDaniel and Beebe[15] applied the Biot theory and computed the acoustic characteristics of different types of sediments. The sediments were classified on the basis of their mean grain size. To derive expressions for the acoustic characteristics they used empirical relations between permeability and grain size. The variation of attenuation with frequency was found to obey the law $\alpha(f)=A f^{n}$ where $n$ lies between 1 and 1.8. The results obtained by them are shown in Figure 2-8. Hovem[16], however, suggests that the viscous losses which is the cause of the nonlinear dependence can be made to vary linearly with frequency if a distribution of grain sizes is adopted.

Figure 2-11 is a collection of laboratory and field data for attenuation plotted against frequency with the prediction of the Biot model as computed by Stoll[23] superimposed. We note that in the low frequency region there is considerable scatter in the experimentally determined values and applicability of the Biot model to marine sediments cannot be confirmed on the basis of the evidence presented in this figure.

The variation of compressional wave with frequency as obtained using the Biot model is shown in Figure 2-9 for sediments of different mean grain size. For coarser materials (low $\phi$ ) the dispersion is appreciable, which is similar to the result obtained by Liu as such materials also have low $\mathrm{Q}$.

\subsubsection{Compressional wave attenuation vs Depth}

The ray diagram in Figure 1-1 shows the rays interacting with the bottom. The loss of energy suffered due to interaction with the bottom will, therefore, 
$-37-$

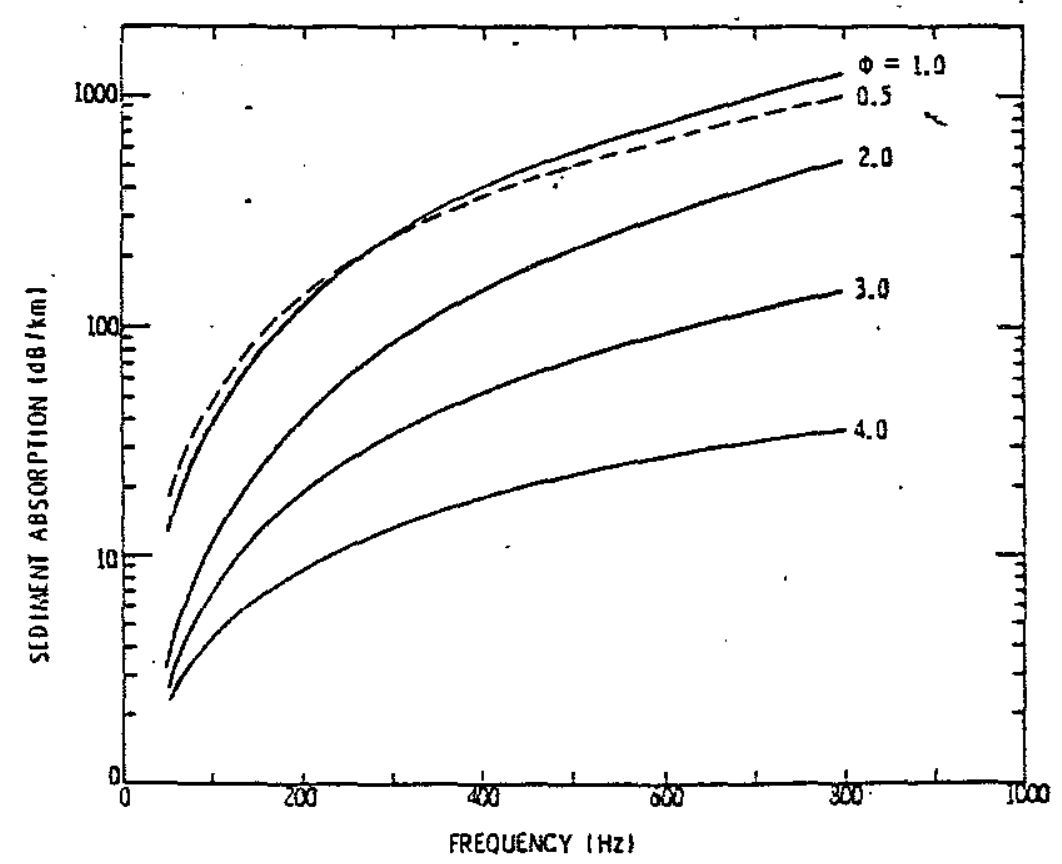

Figure 2-8: Variation of the attenuation coefficient with frequency - reproduced from McDaniel[15] 
$-38-$

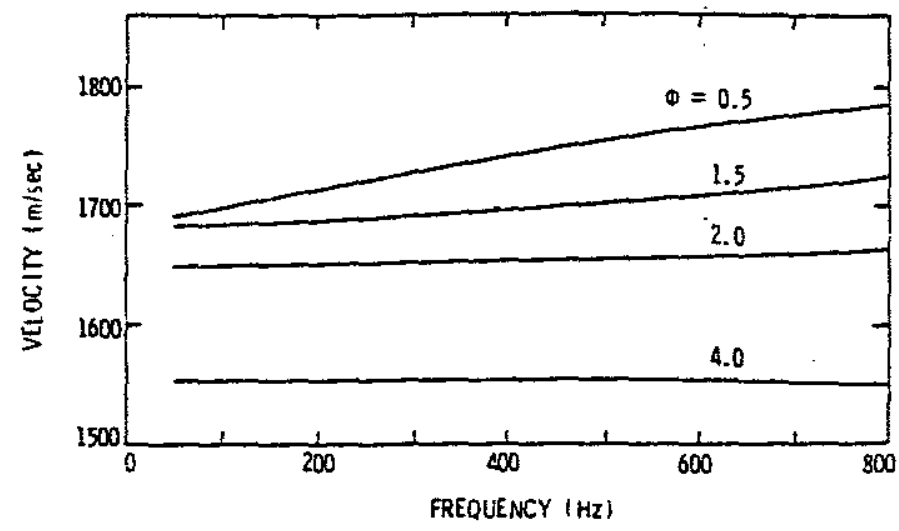

Figure 2-9: Variation of the compressional wave speed with frequencyreproduced from $\mathrm{McDaniel}$ [15] 


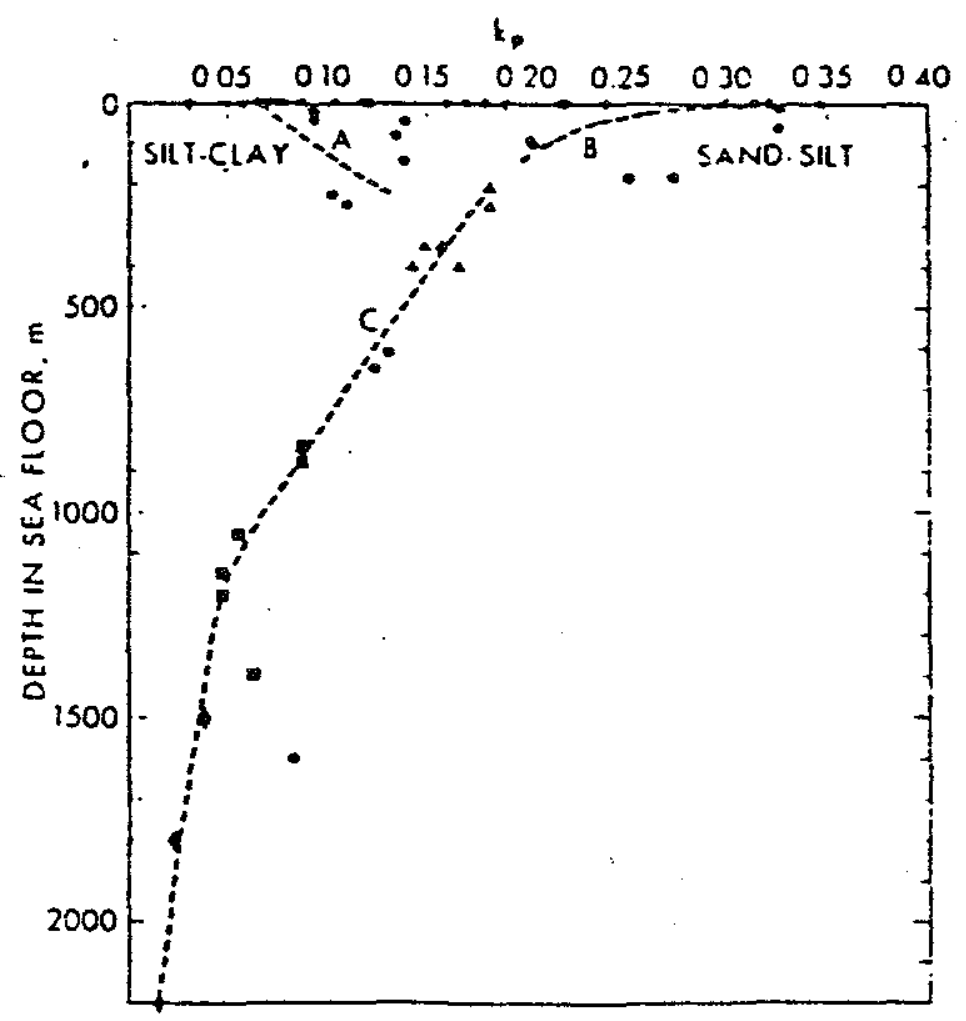

Figure 2-10: Variation of the compressional wave attenuation with depth reporduced from Hamilton[4] 


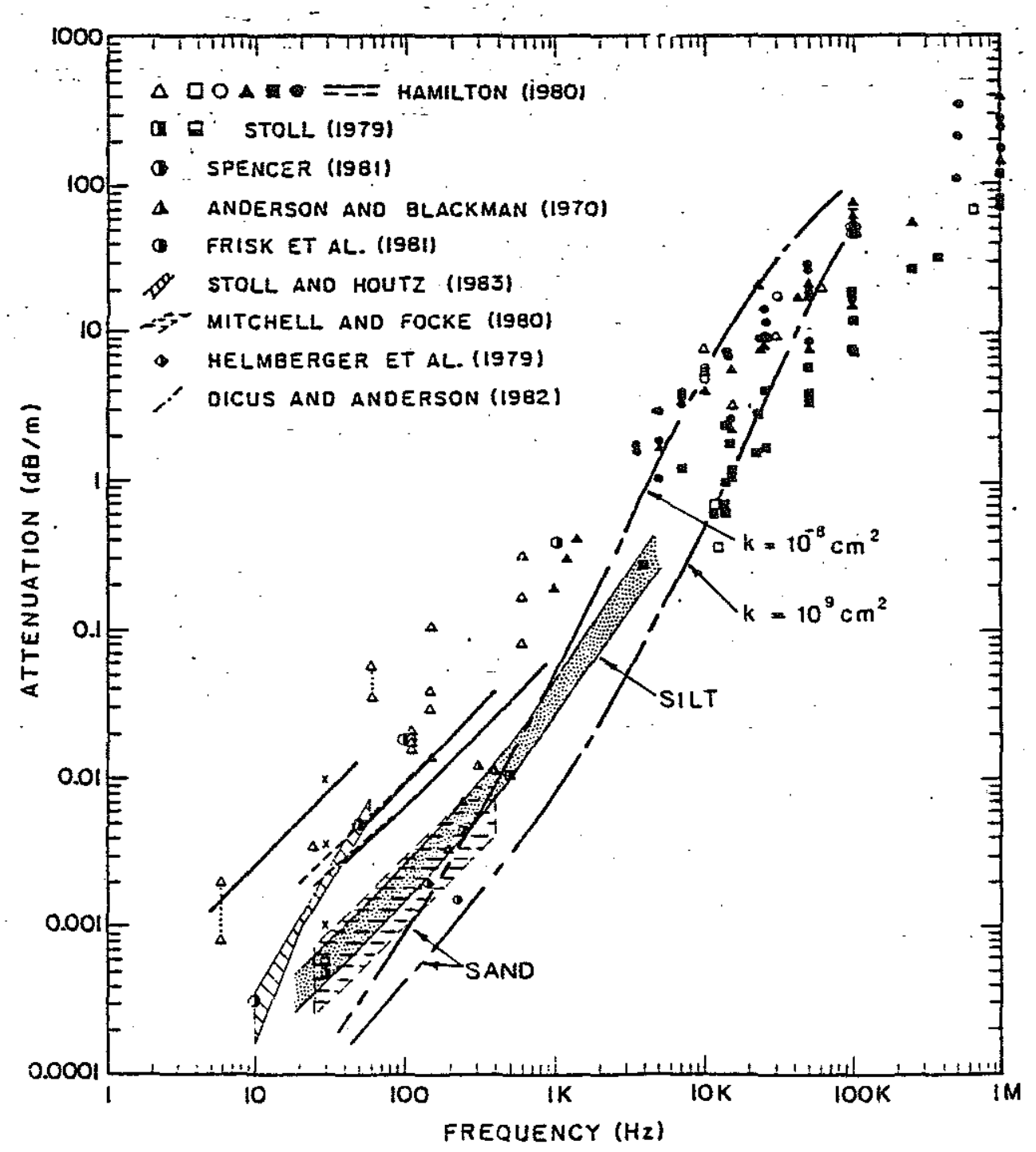

Figure 2-11: Measurements of attenuation coefficient[22] 
depend on the sedimezt layers sampled by these rays. Since each ray has different ray paths the energy loss associated with it will depend on the variation of attenuation with depth. Very little experimental data is available on this important characteristic. The available data is shown in Figure 2-10 which is a plot of $k(z)$. The function $k(z)$ is derived from the attenuation coefficient using the law $\alpha(f, z)=k(z) f$. In the case of silty-clay sediments a peak in the value of attenuation is observed around $300 \mathrm{~m}$. Recent field experiments carried out by Jacobson etall[21] and Mitchell and Focke[19] also indicate the existence of such peaks.

\subsubsection{Conclusions}

The modelling of the sediment as a visco-elastic model or as a porous solid leads to conflicting results especially in the low frequency region. Experimental results in the low frequency region is limited and the question as to which of these models is applicable for marine sediments has not yet been resolved. However, based on the theory presented so far, the following conclusions can be drawn.

1. In the high frequency region (above $1 \mathrm{KHz}$ ), attenuation in low permeability materials can be modelled as having a linear relationship with frequency.

2. The behaviour of marine sediments in the low frequency region is not well understood and under such circumstances it will not be correct to extrapolate the results obtained in the high frequency region to the low frequencies.

3. Dispersion of waves for coarse sediments can be substantial especially over a wide range of frequencies. 


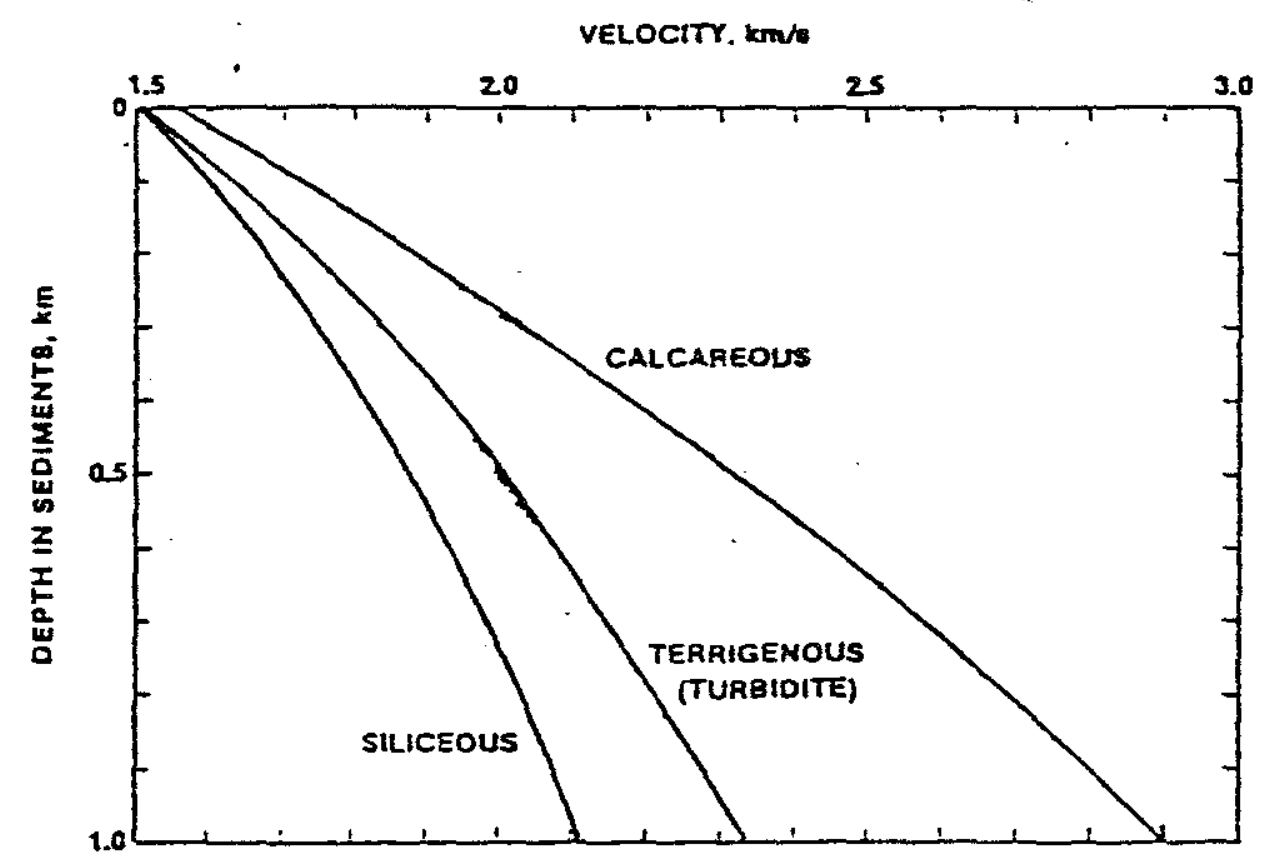

Figure 2-12: Variation of the compressional wave velocity with depth reproduced from Hamilton[4] 


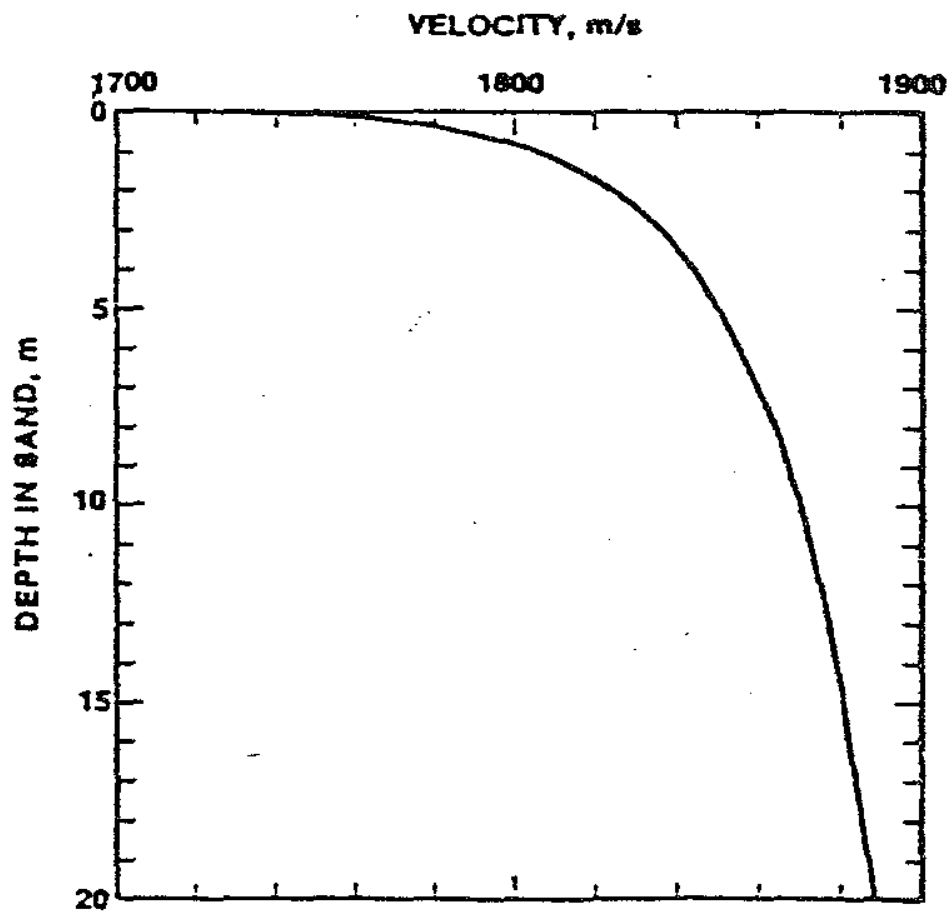

Figure 2-13: Variation of the compressional wave speed with depth in sandy sediments - reproduced from Hamilton[4] 


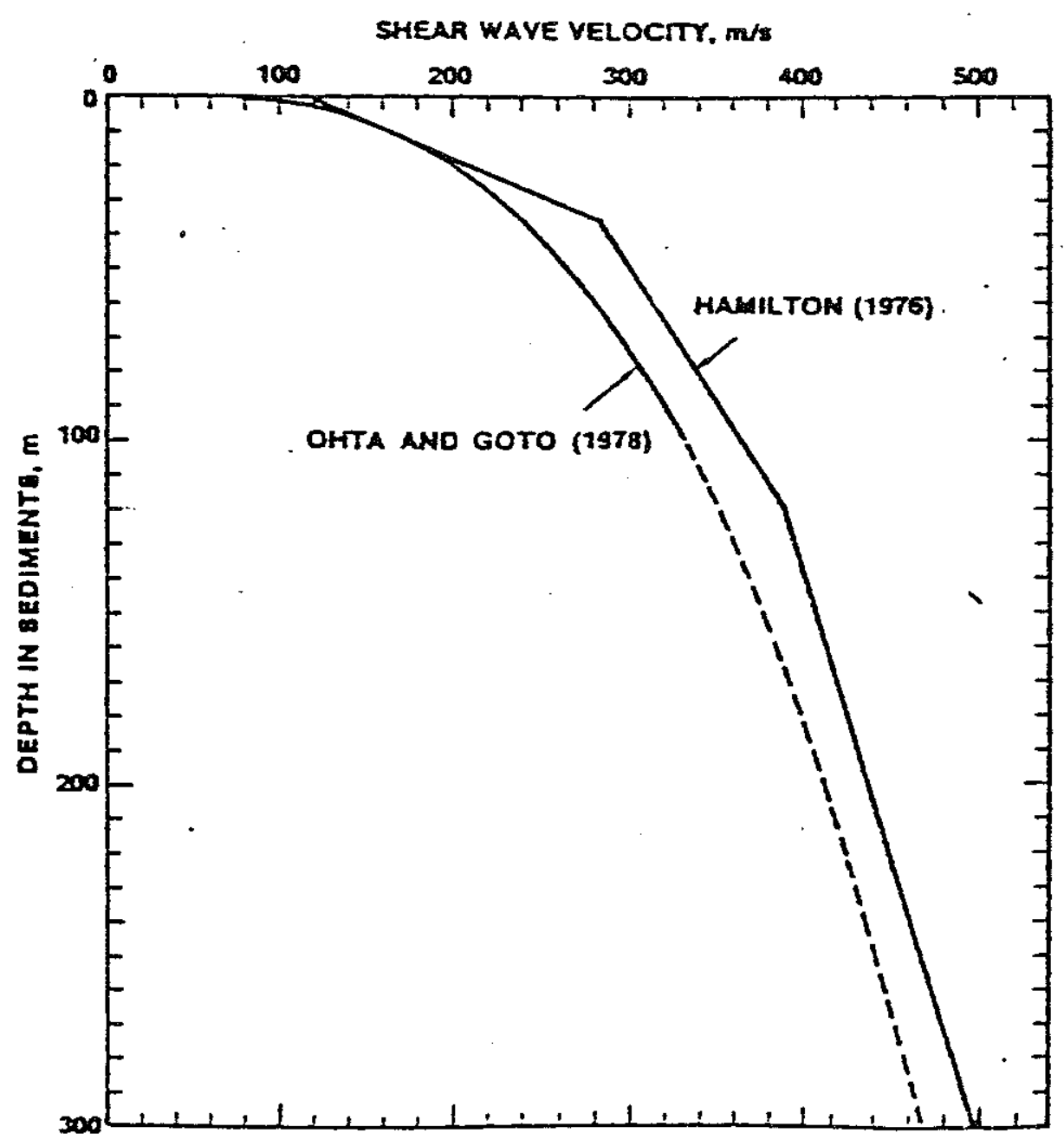

Figure 2-14: Variation of the shear wave speed with depth reproduced from Hamilton[4] 


\subsection{Summary of other essential characteristics of the geoacoustic parameters.}

\subsubsection{Compressional wave speed as a function of depth}

In-situ measurements of velocity at the sediment water interface show a small discontinuity in the compressional wave speed. The velocity ratio defined as the ratio of sound speed in the sediment to the sound speed in water range from 0.97 to 1.03. From the sediment water interface the sound speed increases monotonically. Using experimental data on compressional wave speed at various depths Hamilton[4] gives a regression equation for modelling the compressional wave speed in the sediment. The variation of compressional wave speed with depth is plotted in Figures 2-12and 2-13 for different types of sediments.

\subsubsection{Shear wave speed as a function of depth}

All marine sediments possess enough rigidity to transmit shear waves. Shear waves are important in underwater sound propagation because compressional waves can be partially converted to shear waves at reflection boundaries and by compressional and shear velocity gradients. Variation of shear wave velocity with depth is given in Figure 2-14. For modelling purposes Hamilton[4] gives a regression equation that relates the compressional wave speed to shear wave speed.

\subsubsection{Shear wave attenuation and dispersion}

Experimental results for shear wave attenuation are far fewer than that of compressional waves. For modelling purposes Hamilton[4] suggests the use of the linear relationship between attenuation and frequency in the case of high 
permeability material. In the context of the evidence available for compressional waves the validity of this assumption in the low frequency region is questionable. Since the attenuation coefficient for shear waves is at least an order of magnitude larger than that of compressional waves, dispersion in this case will be substantial.

\subsubsection{Shear wave attenuation as a function of depth}

Very little information is available on the variation of shear wave attenuation

with depth. For modelling purposes Hamilton recommends that the shear wave attenuation be varied with depth in proportion to the variation of compressional wave attenuation with depth.

\subsubsection{Density as a function of depth}

Density of the sediments can be modelled as increasing monotonically with depth due to overburden pressure. Variation of density with depth is in Figure 2-15

\subsection{Ocean bottom model}

In a complete model for the ocean bottom the geoacoustic parameters are functions of the spatial co-ordinates. A simplifying assumption commonly made for the ocean bottom model is that of horizontal stratification i.e., the geoacoustic parameters depend spatially only on depth. Figure 2-16 is a section of the ocean bottom obtained by a $3.5 \mathrm{kHz}$ seismic profiler in the Icelandic Basin[22]. The well defined layering of the bottom can be seen in this figure and such layering is observed in many regions of the ocean. Thus the assumption of horizontal straticication is frequently satisfied. 


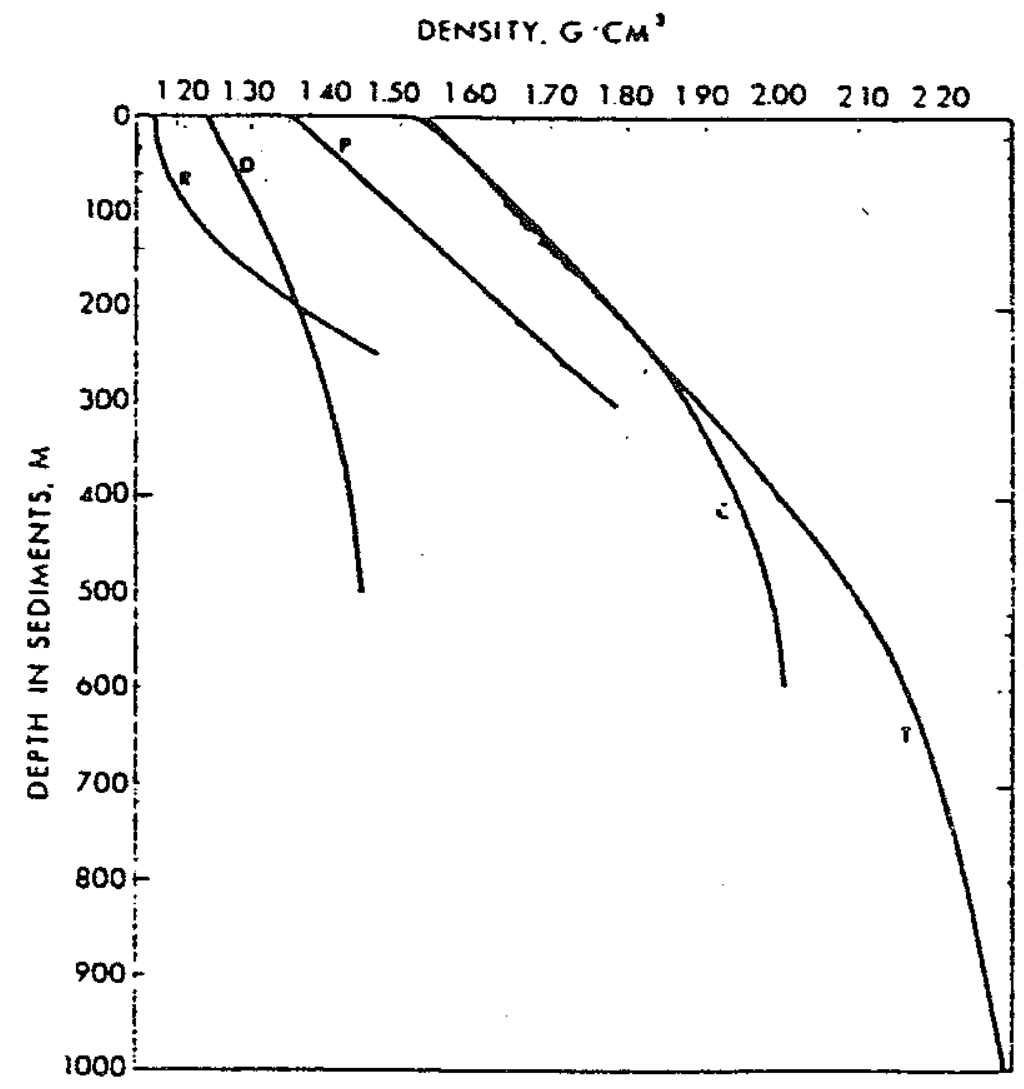

Figure 2-15: Variation of density with depth reproduced from Hamilton[4] 


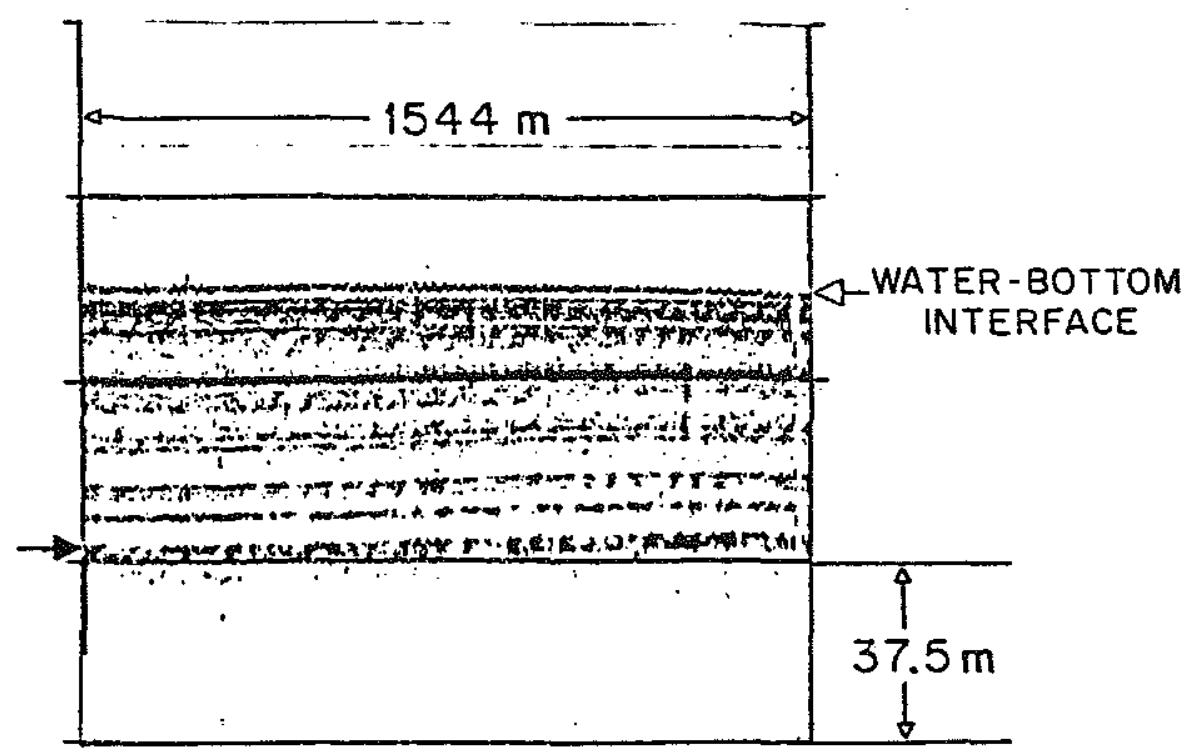

Figure 2-16: Seismic profiling of the ocean bottom 
$-49-$

Water Column

$P_{0}, C_{0}$

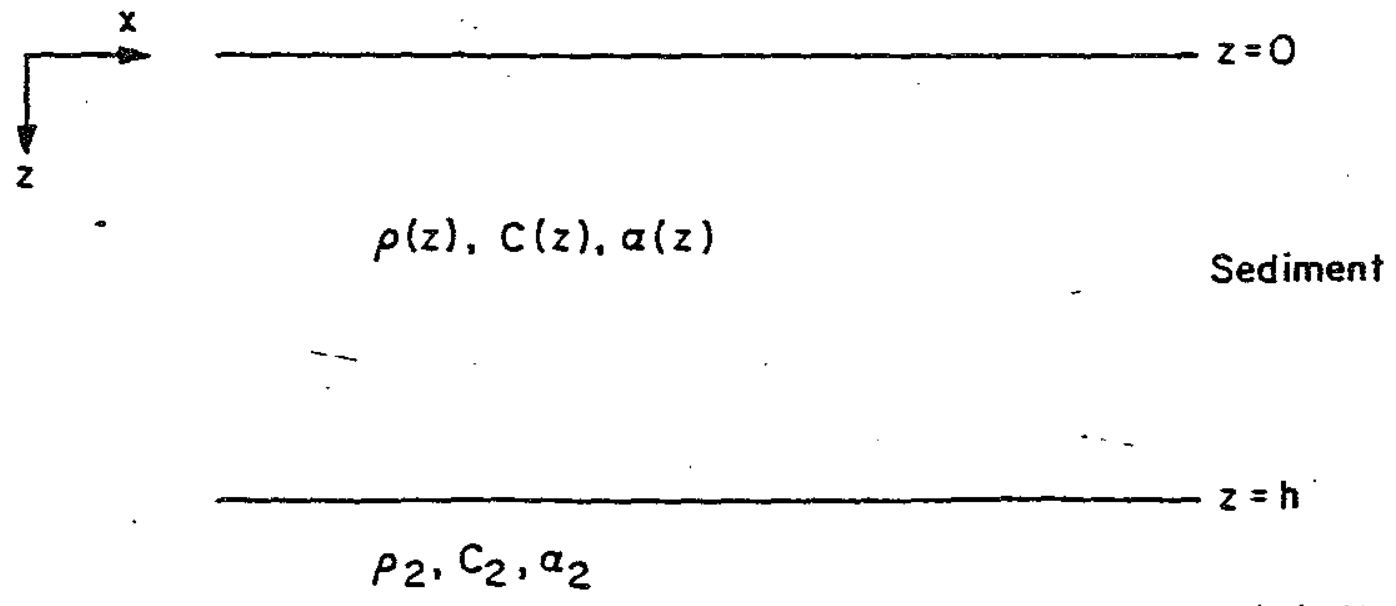

Sub-bottom

Figure 2-17: Ocean bottom model 
Marine sediments have enough rigidity to transmit shear waves. When compressional waves are incident on the ocean bottom interface, shear conversion takes place. If the ocean bottom is modelled as a set of layers with velocity gradients within each layer, then such shear conversion will occur at the layer interfaces and if the velocity gradients are large it will occur continuously within the layers. Fryer[17] studied the effect of shear in marine sediments and concludes that the effect of shear conversion within the the sediment is small at frequncies above $20 \mathrm{~Hz}$. However, if strong discontinuities in shear speeds exist or if any energy reaches the basement where the shear speed becomes comparable to the compressional wave speed, then shear conversion cannot be ignored. For thick sediments, we can assume that there are no strong discontinuities in the shear speed within the sediment and very little energy reaches the basement. Under such assumptions, the effect of shear conversion can be ignored and the sediment treated as a fluid. The ocean bottom model now contains only three parameters, the compressional wave speed, compressional wave attenuation and density.

Some investigators have made a further simplifying assumption that the dispersion of compressional waves is negligible and therefore the compressional wave speed is a function of depth alone. For reasons explained earlier we will not make such an assumption. Further we do not assume linear dependence of attenuation with frequency since the applicability of this behaviour at low frequencies to all types of sediments is in doubt.

The model for the ocean bottom we adopt is shown in figure2-6. The figure represents a thick sediment layer in which the compressional wave speed, density and attenuation can vary arbitrarily with depth, overlying a sub-bottom of constant density, compressional wave speed and attenuation. Since we assume that very little energy reaches the basement, the sub-bottom is modelled as an infinite 
$-51-$

half space. In the water column for the purpose of our investigation we assume that the density and sound speed are constant. The negligible attenuation in the water is ignored. 


\subsection{References}

1. Keiiti Aki, and Paul.G.Richards, Quantitative Seismology:Theory and Methods,vol.1,(Freeman, San Francisco, 1980).

2. Edwin L.Hamilton, "Compressional wave attenuation in marine sediments," Geophysics, 37(4), 620-646 (1972).

3. H.Kolsky, Stress Waves in Solid,(Dover, NewYork, 1963)

4. Edwin L.Hamilton,"Geoacoustic modelling of the sea floor," J. Acoust. Soc. Am., 68(5), 1313-1340 (1980).

5. B. Gross, Mathematical Structure of the Theories of Viscoelasticity,(Hermann, Paris, 1053).

6. Hsi-Ping Liu, D.L. Anderson, and Hiroo Kanamori,"Velocity dispersion due to an elasticity ; implications for seismolgy and mantle composition," Geophys. J. R. Astr. Soc., 47, 41-58 (1976).

7. Lloyd.D.Hampton,"Acoustic properties of sediments," J. Acoust. Soc. Am., 42(4), 882-890 (1967).

8. Sh.A. Azimi, A.V. Kalinin,V.V. Kalinin, and B.L.Pivovarov,"Impulse and transient charecteristics of media with linear and quadratic absorption laws," Isvestiya, Physics of the solid earth, 88-93, (1968).

9. C. Lomnitz, "Linear dissipation in solids," Journal of Applied Physics, 28, 201-205 (1957).

10. M.A.Biot,"Theory of elastic waves on a fluid - saturated porous solid I. Low frequency range," J. Acoust. Soc. Am., 28, 168-178 (1965).

11. M.A.Biot,"Theory of elastic waves on a fluid -saturated porous solid II. High frequency range," J. Acoust. Soc. Am., 28, 179-191 (1865).

12. M.A.Biot, "Generalised theory of acoustic propogation in porous dissipative media," J. Acoust. Soc .Am., 34, 1254-1264 (1962).

13. Robert.D.Stoll, "Acoustic waves in saturated sediments," in Physics of sound in marine sediments,(Plenum, NewYork, 1974). 
14. R.D.Stoll, and G.M.Bryan,"Wave attenuation in saturated sediments," J. Acoust. Soc. Am., 47, 1440-1447 (1970).

15. S.T.McDaniel, and J.H.Beebe,"Semi-emperical models based on the Biot theory," in Acoustics and the sea bed,(Bath U. P, Bath, 1983).

16. J.M.Hovem, "Attenuation of sound in marine sediments," in Bottom interacting ocean acoustics,(Plenum, NewYork, 1980).

17. G.Fryer" Reflectivity of ocean bottom at low frequency," J. Acoust. Soc. Am., 63(11), 35-43, (1978).

18. G.V. Frisk, J.A. Doutt and E.E. Hays," Bottom interactions of low frequency acoustic signals at small grazing angles in the deep ocean," J. Acoust. Soc. Am., 69, 84-94 (1881).

19. S.K. Mitchell, and K.C. Focke," New measurement of compressional wave attenuation in the deep ocean sediments," J. Acoust. Soc. Am., 67(5), 1582-1589 (1980).

20. R.D. Stoll, and R.E. Houtz, " Attenuation measurements from sonobuoys," J. Acoust. Soc. Am., 73(11), 163-172 (1983).

21. R.S.Jacobson, G.G. Shor Jr., and L.M. Dorman," Linear inversion of body wave data- Part II, Attenuation versus depth using spectral ratios," Geophysics, 46, 142-152 (1981).

22. G.V.Frisk, J.A. Doutt, and E.E. Hays, "Geoacoustic models of the Icelandic Basin," Submitted to the J. Acoust. Soc. Am. (1985).

23. R.D. Stoll, "Marine sediment acoustics," J. Acoust. Soc. Am., 77(5), 1789-1799 (1985). 


\section{Chapter 3 \\ Determination of the Attenuation Profile}

Attenuation as described in Chapter 2 refers to the decrease in the amplitude of propagating waves due to a variety of energy damping mechanisms. This attenuation, also called intrinsic attenuation, depends on the type of sediment (i.e the compositon of the sediment, the particle size and other parameters). Laboratory measurements of intrinsic attenuation can be readily performed but are restricted to high frequencies because of the long wave lengths at low frequencies and the difficulty in eliminating boundary effects. Laboratory measurements have, therefore, been limited to frequencies above $1 \mathrm{KHz}[1-5]$.

In view of this, field experiments which utilize the in-situ deposits of sediments as large scale specimens have been performed. In such field experiments it is necessary to take into account all factors like spreading, reflections, etc. which affect the amplitude of the propagating waves. The determination of the attenuation coefficient structure from field experiments, even in the simplest of geological setting is, therefore, a difficult task.

In this chapter we will review some of the methods proposed in the literature for obtaining the attenuation profile for the bottom from field measurements. We then describe a field experiment that has been proposed by Frisk etal[6] to obtain the plane-wave reflection coefficient for the ocean bottom as a function of the angle of incidence. The sequence of processing steps that can be adopted to obtain the acoustical properties of the bottom starting with the plane-wave reflection coefficient information is then indicated. The advantage of this method for the 


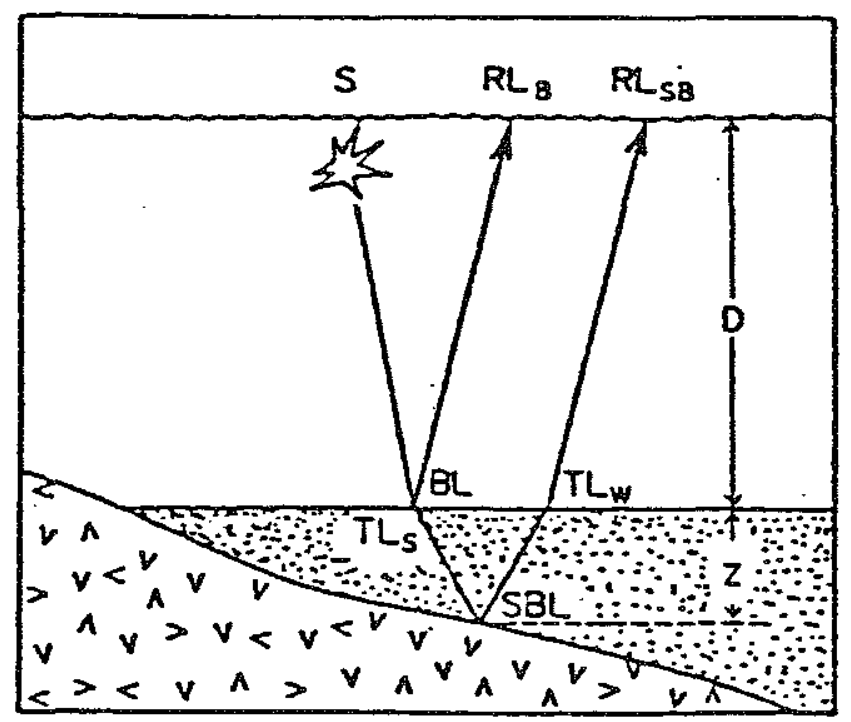

Figure 3-1: Configuration of experiment by Anderson and Blackman[7]

$\alpha(\mathrm{f})=$ Attenuation coefficient $(\mathrm{dB} / \mathrm{m})$ of sediment

$\alpha_{\omega}(\mathrm{f})=$ Attenuation coefficient of water

$\mathrm{BL}=$ Bottom loss of bottom reflected signals in decibels

$\mathrm{D}=$ Depth of water

$\mathrm{z}=$ Thickness of sediment wedge

$\mathrm{RL}_{\mathrm{sb}}(\mathrm{f})=$ Peak amplitude of sub-bottom reflected signal

$S(f)=$ Source level in decibels

SBL $=$ Sub-bottom loss

$\mathrm{TL}_{\mathrm{s}}=$ Transmission loss of signal going through the water/sediment interface

$\mathrm{TL}_{\omega}=$ Transmission loss of signal going through the sediment water interface 
determination of the attenuation profile over the others described in this chapter will also be presented. We conclude the chapter by a statement of the problem that will be considered in the remaining part of this thesis and the assumptions that will be made in solving the problem.

\subsection{Review of methods in the literature}

Anderson and Blackman[7] describe a method for determining the average attenuation coefficient from insitu measurements. A requirement for the experiment is that the sediment layer be wedge-shaped and overly a highly reflective bottom. The schematic diagram in Figure 3-1 shows such a configuration. In such an environment, the field experiment is performed to obtain an average value of attenuation for the sediment. Assuming near normal incidence, the pressure pulse due to the source interacts with the water-sediment interface first. Part of the energy is returned to the water column while the remaining is transmitted into the sediment. This transmitted energy again undergoes reflection and transmission at the sediment sub-bottom interface. Part of this reflected energy is returned to the water column. If $R L_{s}$ and $R L_{s b}$ are the received pressure levels of the sediment and sub-bottom reflected signals, they can be related to the source level, spreading losses and other losses by the following equations. The difference in the field returned from the water-sediment interface and sedimentsub-bottom interface can then be related to the attenuation in the sediment. The symbols used in the following equations are explained in the legend to figure 3-1.

$$
\begin{aligned}
& R L_{b}=S-B L-20 \log 2 D-2 \alpha_{\omega} D \\
& R L_{s b}=S-T L_{b}-S B L-T L_{\omega}-2 \alpha z-20 \log (D+z)-2 \alpha_{\omega} D
\end{aligned}
$$


$-57-$

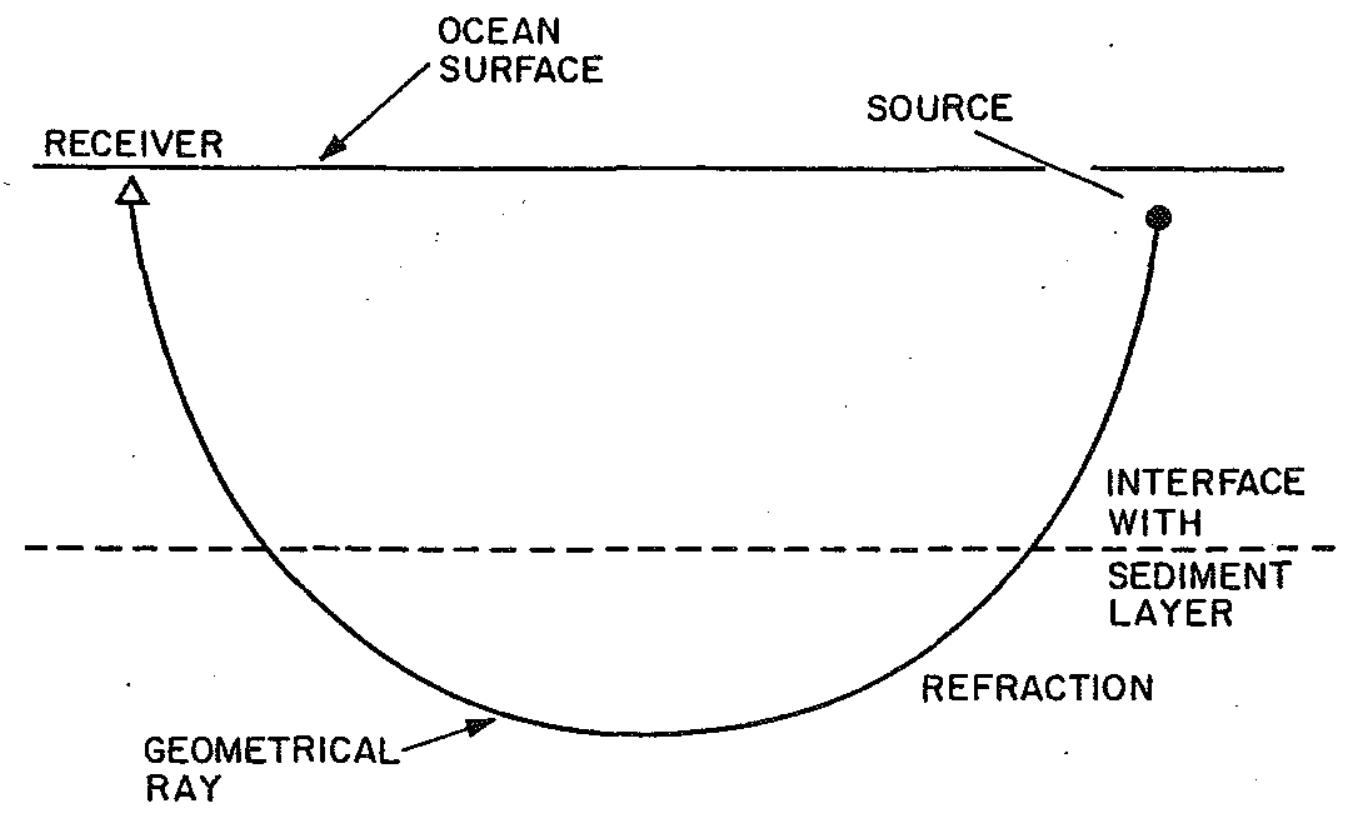

Figure 3-2: Configuration of experiment by Jacobson etal[8] 
Subtracting, we obtain

$$
\begin{aligned}
R L_{b}-R L_{s b}=S B L & +\left[T L_{\omega}+T L_{s}-B L-20 \log 2 D\right] \\
+ & 2 \alpha z+20 \log (D+z) .
\end{aligned}
$$

Assuming that the term within the bracket remains constant for the entire wedge, we obtain

$$
R L_{b}-R L_{s b}-20 \log 2(D+z)-S B L=\alpha(2 z)+c
$$

Ignoring any contribution due to spreading term, i.e. $20 \log 2(\mathrm{D}+\mathrm{z})$, and any change in the sub-bottom reflectivity, this equation reduces to

$$
R L_{b}-R L_{s b}=\alpha(2 z)+c
$$

By measuring $\left(R L_{b}-R L_{s b}\right)$ at different points in range, i.e. at different depths in the bottom, this quantity is plotted as a function of depth. The slope of the line obtained gives the attenuation coefficient. The depth at each observation point is determined from measuring the arrival times of the two reflected signals and knowing the sound speed in the bottom.

The spectral ratio method of Jacobson, Shor, and Dorman[8] uses seismic refraction data to determine attenuation as a function of depth. The experimental configuration is shown in Figure 3-2. Different ray paths from the source sample different depths in the ocean bottom. Assuming horizontal stratification, the amplitude spectrum of the received signal waveform can be written as

$$
A_{j}(\theta, f, z)=E_{0 j}(\theta, f) F_{j}(\theta, f, z) I(f)
$$

where $\theta$ is the angle of incidence, $f$ is the frequency, and $z$ the $\operatorname{depth} . A(\theta, f, z)$ is the 
received signal, $E(\theta, f)$ is the source signal, $F(\theta, f, z)$ the earth response and $I(f)$ the instrument response. The suffix $j$ refers to the $j$ th observation. The earth response function $F_{j}(\theta, z, f)$ can be written as

$$
F_{j}(\theta, f, z)=T_{j}(\theta, z) R_{j}(\theta, z) G_{j}(f, z)
$$

where $T$ is the function containing transmission and reflection coefficients, scattering loss, shear conversion etc., $R$ is the function representing spreading loss and $G$ is the attenuation operator. $T$ is assumed to be independent of frequency. The frequency dependence of $G$ is given by

$$
G(f, z)=\exp \left(-k(z) f^{n} P L(z)\right)
$$

where $P L(z)$ is the path length, $f k(z)$ is the attenuation coefficient. If $E$ and $I$ are known, the earth response $F$ can be obtained. To get a good estimate of the source function, the direct water borne energy is measured. This is given by

$$
A_{w \jmath}\left(\theta, f, z_{w}\right)=E_{0}(f) R_{w \jmath}\left(\theta, z_{w}\right) I(f)
$$

where $R_{w j}$ is the spreading loss function ralated to the waterborne path. The spectral ratio defined as the logarithm of the ratio of the refracted signal amplitude to the direct water signal is then obtained.

$$
\frac{A_{j}(\theta, f, z)}{A_{w j}\left(\theta, f, z_{w}\right)}=\frac{T_{j}(\theta, z) R_{j}(\theta, z) G(f, z)}{R_{w j}\left(\theta, z_{w}\right)}
$$

Taking the logarithm of both sides, the spectral ratio is 
$-60$

$\checkmark$

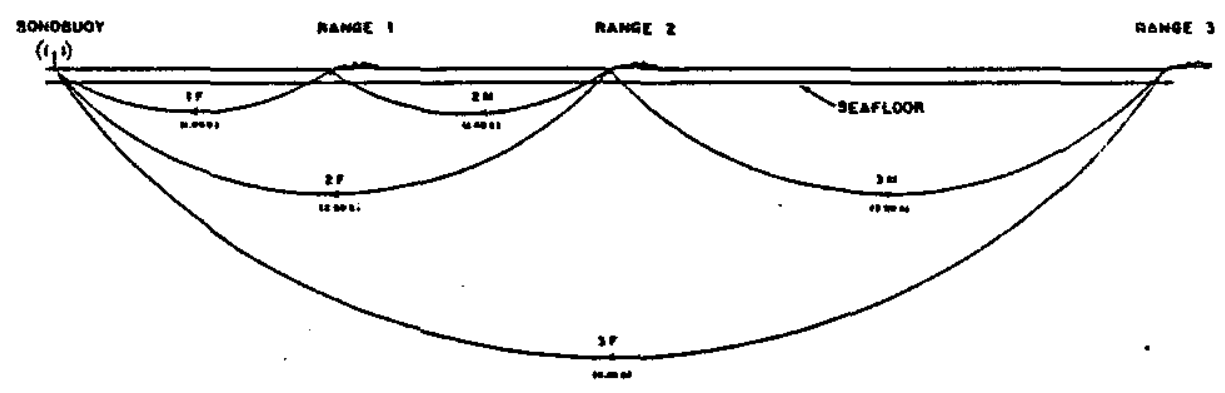

Figure 3-3: Configuration of experiment by Stoll[11] 


$$
\begin{aligned}
S R(\theta, f, z)=\ln _{j}(\theta, z)+ & \ln R_{j}(\theta, z)- \\
& \ln R_{w j}(\theta, z)+\ln G_{j}(f, z)
\end{aligned}
$$

Assuming that the attenuation cofficent is linearly related to frequency

$$
\ln G(f, z)=-k(z) f P L(z)
$$

Then,

$$
S R_{j}(\theta, f, z)=S R_{j 0}(\theta, z)-\sum_{i=1}^{N} k_{i} f P L_{i j}
$$

where,

$$
S R_{\jmath 0}=\ln T_{j}(\theta, z)+\ln R_{j}(\theta, z)-\ln R_{w j}(\theta, z)
$$

By plotting $S R(\theta, f, z)$ with respect to $f$ we can obtain the intercept $S R_{0}$. Then,

$$
\frac{S R_{j}-S R_{j 0}}{f}=-\sum_{i} k_{i} P L_{i j}
$$

where $S R_{0}$ is the intercept, $P L_{i j}$ is the path length in the ith layer for the $j$ th observation. A linear inverse method is now used to obtain $k_{i}$. Having obtained $k_{i}$, the attenuation in the ith layer equals $f k_{i}$. Wrolstad[9] also uses a similar method for determining the attenuation profile. The spectral ratio method has been applied by Hauge[10] to vertical seismic profiles to obtain attenuation information.

Stoll and Houtz[11] utilize the fact that in certain areas of the ocean where there are deep sediment layers, the velocity profile is smooth and linear. With linear profiles the refracting paths in the sediment layers will be arcs of a circle. The experiment is similar to a refraction experiment and the configuration is shown in Figure 3-3. The amplitude of the signal received at the time of the first arrival or 
$-62-$

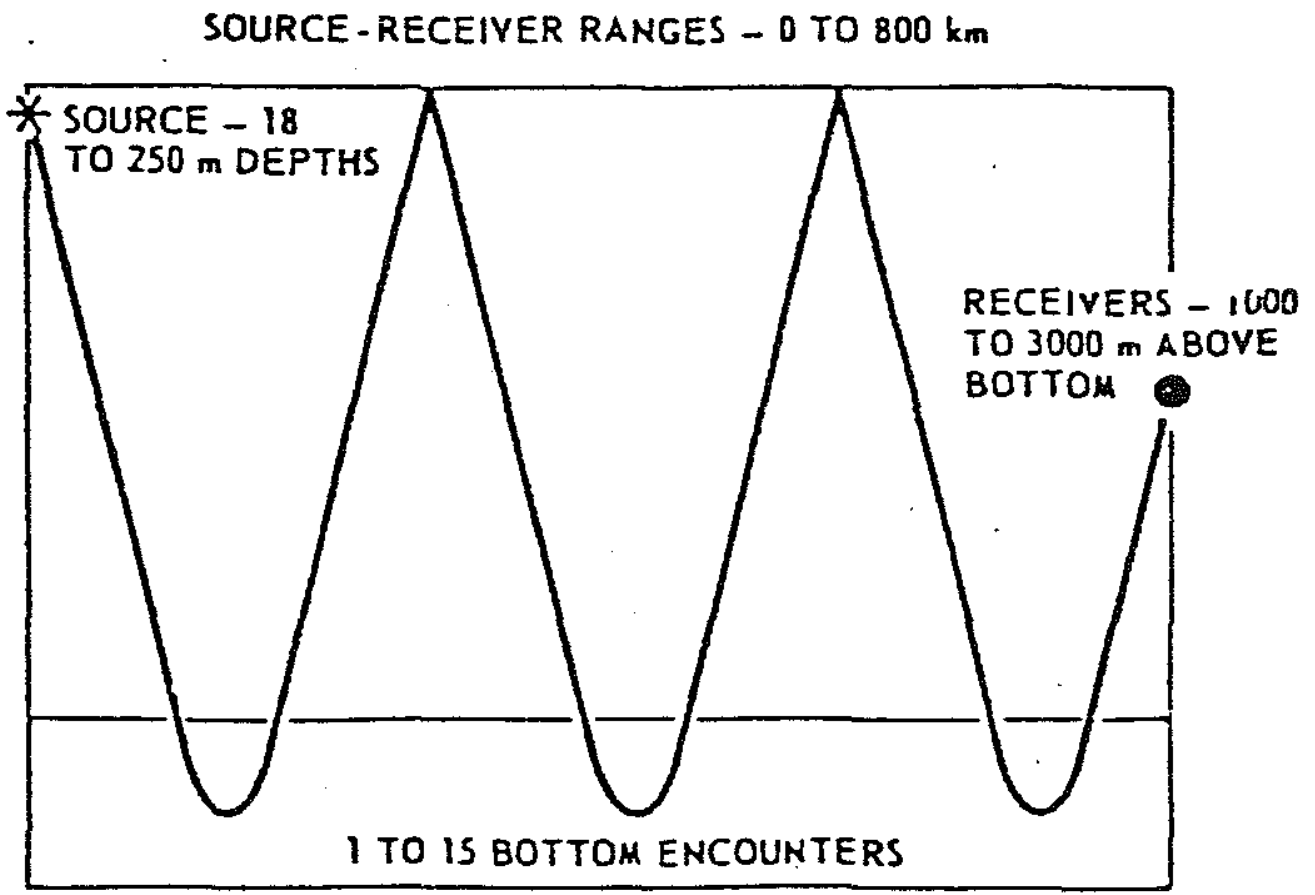

Figure 3-4: Configuration of experiment by Mitchell and Focke[12] 
at a multiple of this time is given by

$$
A_{n}=A_{s}(\theta) \frac{1}{1+n}\left(\frac{\tan (\theta)}{S(x, \theta)}\right)^{1 / 2}\left(1-R^{2}\right)(\theta)^{1+n} \exp \left(-\Phi_{n}(\theta)\right)
$$

where,

$$
S(x, \theta)=x \frac{d x}{d \theta}
$$

where $n=0$ for the first the first arrival and $n=1,2, .$. for the first multiple and so on. The term in parenthesis accounts for geometrical spreading, $R(\theta)$ is the reflection coefficient and $A(\theta)$ is the amplitude of the source. $\theta$ is the angle of inclination of the angle at the source and $(1+n) x$ is the range between source and receiver. The exponent $-\Phi(\theta)$ is the total loss of amplitude occuring due to intrinsic attenuation, scattering and other causes and may be expressed in terms of the attenuation coefficient by the expression given below.

$$
\Phi_{n}=(n+1) \sum_{s} \alpha(f) \Delta s
$$

To evaluate $\Phi$, we form the ratio $A_{1} / A_{0}$,

$$
R_{a}=\frac{A_{1}}{A_{0}}=\frac{1}{2}\left(1-R^{2}\right) \exp \left(-\Phi_{0}\right)
$$

Therefore,

$$
\Phi_{0}=-\ln \left(\frac{2 R_{a}}{\left(1-R^{2}\right)}\right)
$$

and knowing $\Phi_{0}, \alpha(f)$ is determined.

Mitchell and Focke[12] use the setup shown in Figure 3-4 for their field 
$-64-$

experiment. One of the ray paths from the source to the receiver is shown in the Figure 3-4. There may be many more such paths. The major part of the loss of signal energy occurs along portions of the ray that lie in the sediment. This loss, when normalised to a single bottom encounter and expressed as a function of grazing angle is the bottom reflection loss. Obtaining this is the first step in this method. Mitchell and Focke then use the fact that for rays penetrating the bottom at high angles of incidence, the bottom loss associated with them is largely due to attenuation in the layers. Under such assumptions, the bottom loss can be expressed as a function of the attenuation coefficient and the path length in the bottom. When the attenuation is a function of depth, then this relationship is given by the equation below.

$$
B L=f \int_{L} k(l) d l
$$

Here we have assumed that $\alpha(f, z)=k(z) f$ and the integral is over the path length. Using this and considering a number of rays all at large angles of incidence a matrix of the form given below can be constructed.

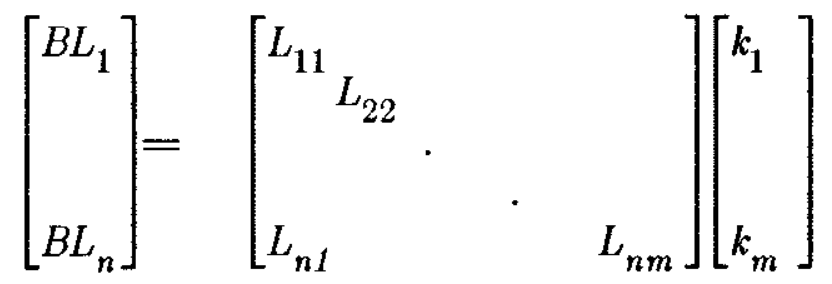

Here $L_{i j}$ is the path length in the $j$ th layer of a ray at an angle of incidence of $\theta_{i}$. To compute the path length it is assumed that the sound speed in the bottom is known. This matrix, which is lower triangular, can be easily inverted to give $k_{i}$ in different layers and as before the attenuation coefficient in each layer can thus be obtained. 
$-65-$

Spofford[13] describes a method of determining the sound speed gradient and an average value of the attenuation coefficient for the sediment from a knowledge of the bottom loss expressed as a function of the angle of incidence. Such data exhibit an abrupt increase in loss at a grazing angle corresponding to the development of a minimum range caustic in the bottom refracted path. If this grazing angle is obtained from the data, then using a relationship between this angle and the sound speed gradient for the bottom, the sound speed gradient is determined. The knowledge of the sound speed gradient gives the path lengths of the refracted rays. The attenuation coefficient value is then obtained from the difference in levels between the bottom reflected and refracted rays.

The methods described above make some or all of the following assumptions.

1. The reflection and transmission coefficients are independent of frequency.

2. The attenuation coefficient is linearly related to frequency and therefore $\alpha(\mathrm{f}, \mathrm{z})$ can be written as $\alpha(f, z)=k(z) f$.

3. The compressional wave speed for the medium is known.

4. The compressional waves are dispersionless.

5. Where other loss mechanisms like scatter,shear conversion are involved, the attenuation coefficient obtained will be the 'effective attenuation' and not the 'intrinsic attenuation'.

The field measurement of attenuation coefficent show considerable scatter as seen in Figure 2-11. On the basis of the data presented in this figure, it is clear that extrapolation of the high frequency results into the low frequency region using linear law will be in error. Further, the data in the low frequency range are spread over a wide range of values of attenuation and there is no consistent pattern to justify a particular frquency dependence. 
$-66-$

We noted in Chapter 2 that both the theoretical models predict substantial dispersion for sediments with high attenuation coefficent. In view of this the assumption that the medium is dispersionless is questionable. This is an important consideration because all methods described above assume that the compressional wave velocity profile for the medium is known and this information is used in the determination of the attenuation coefficient. Therefore any errors in the compressional wave speed arising out of this assumption will manifest as errors in attenuation coefficient.

These considrations indicate the desirability of an experiment which uses a monochromatic source for the determination of the acoustic parameters of marine sediments. Experimental configuration of one such field measurement carried out by Frisk etal is shown in Figure 3-5. Two receivers are moored on the ocean bottom at heights $1.17 \mathrm{~m}$ and $54.55 \mathrm{~m}$ from the bottom. A CW source is suspended from the ship at the end of a long cable. The ship is then allowed to drift and as the ship drifts away slowly the two receivers record the pressure field. By suitable signal processing methods the pressure field as a function of range is obtained.

Since the recorded pressure field at the receiver depends on the acoustic parameters of the bottom, these parameters can be obtained by forward modelling. In this we assume an initial guess model for the acoustic parameters and solve the direct problem to obtain the pressure field at the receiver as a function of range. This is compared with experimentally determined value, and the acoustic parameters are changed until good fit between the experimentally determined value and the theoretically determined value is obtained. Frisk[6] has done such analysis with considerable success and obtained geo-acoustic models, including an average attenuation coefficient, for different regions of the ocean. Frisk[6] also describes how an estimate of the attenuation coefficient is obtained from the recorded 


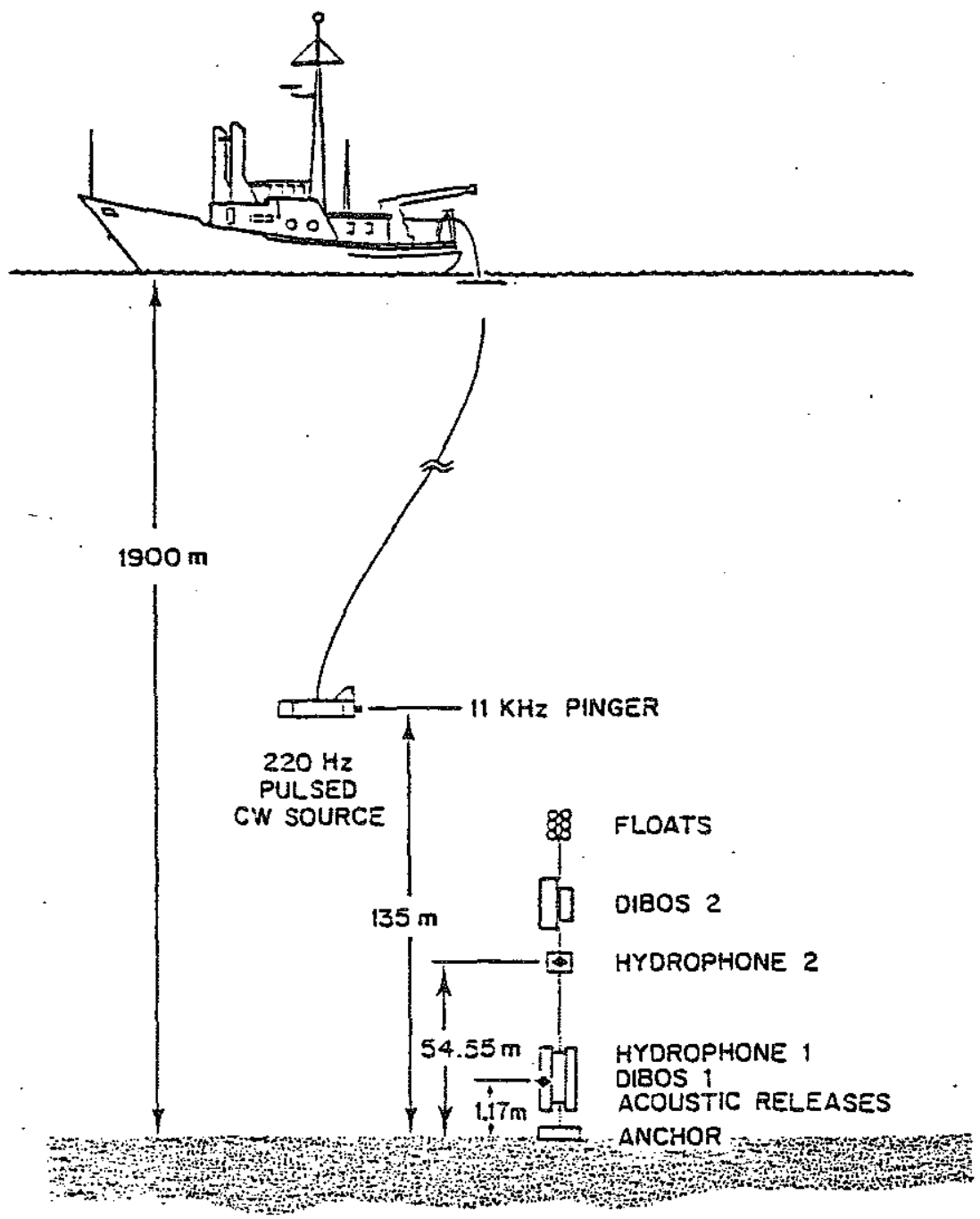

Figure 3-5: Configuration of experiment by Frisk etal[6] 
pressure field by studying the peak to null difference in the measured interference pattern.

\subsection{Determination of the attenuation profile from the plane-wave reflection coefficient}

By suitably proceessing the measured pressure field, the plane-wave reflection coefficient for the ocean bottom can be obtained. The plane-wave reflection obtained is a function of angle of incidence and it contains all the information about the acoustic parameters of the bottom. The determination of the acoustical properties of the bottom from the plane-wave reflection coefficients is a more exact. method and we will follow this path.

A possible sequence of steps to obtain the complete set of acoustic parameters starting with the measured field is given in Figure 3-6. We will briefiy indicate the approaches that can be adopted for solving each step.

\subsubsection{Determination of plane-wave reflection coefficient(step 1)}

The total pressure field is the sum of the direct field and the reflected field. After subtracting out the direct ficld, the reflected field is given by[14];

$$
P_{r e f}(r)=\int_{0}^{\infty} \frac{i}{k_{z 0}} R\left(k_{r}\right) J_{0}\left(k_{r} r\right)\left\{\exp \left(i k_{z 0}\left(z_{r}+z_{s}\right)\right)\right\} k_{r} d k_{r}
$$

where $R\left(k_{r}\right)$ is the plane wave reflection coefficient for the bottom, $k_{r}$ is the horizontal wave number, $r$ is the range, $k_{z 0}$ is the vertical wave number in the water column and $z_{r}$ and $z_{s}$ are receiver and source heights from the bottom. $J_{0}$ is the zeroeth order Bessel fuction. Alternatively we can relate the reflection coefficient to 


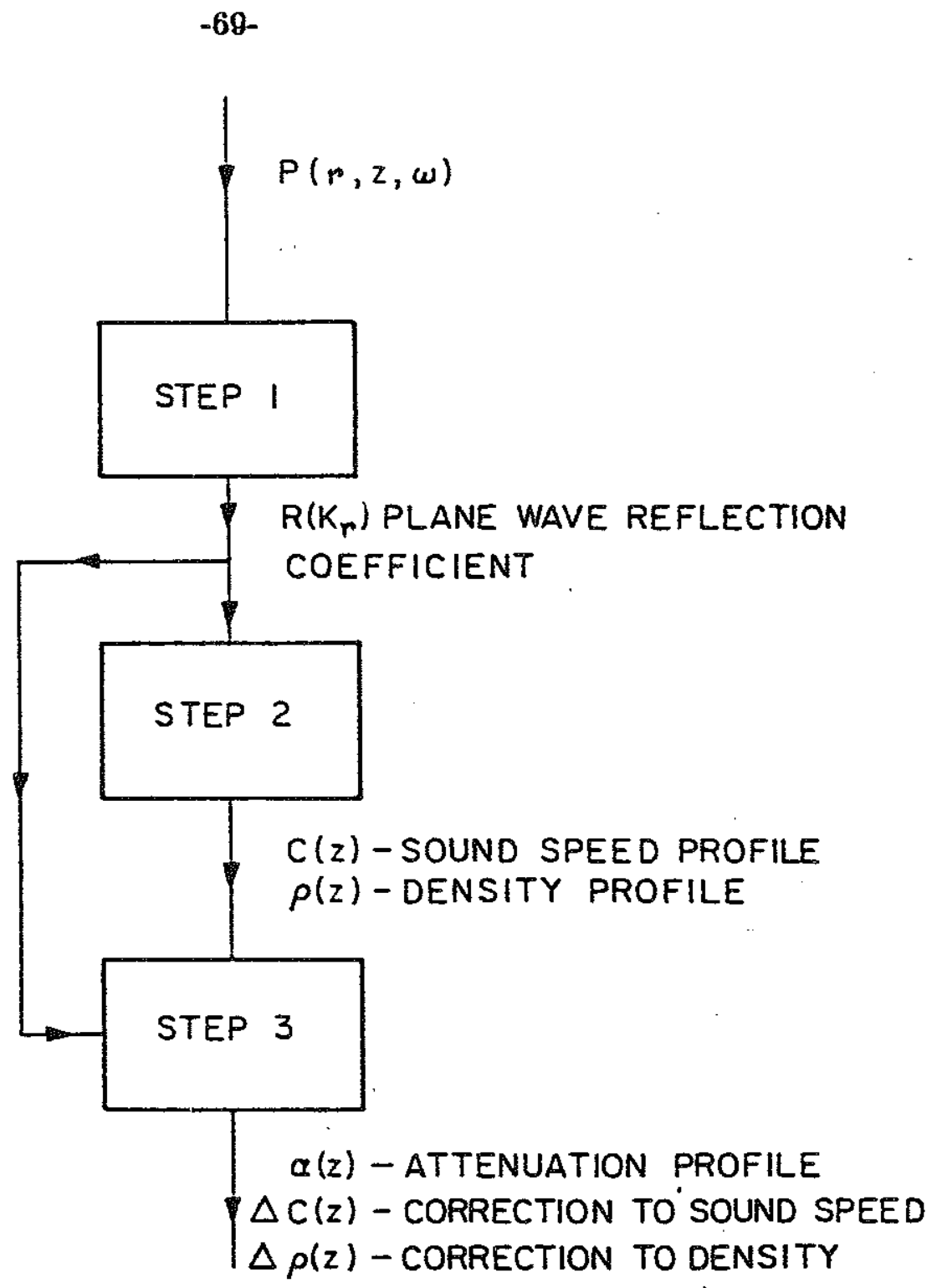

Figure 3-6: Steps for obtaining geoacoustic parameters from pressure field 
the pressure field by the follwing equation.

$$
\frac{i}{k_{z 0}} R\left(k_{r}\right)\left\{\exp \left(i k_{z 0}\left(z_{r}+z_{s}\right)\right)\right\}=\int_{0}^{\infty} P_{r e f}(r) J_{0}\left(k_{r} r\right) r d r
$$

Therefore, by performing the Hankel transform of the reflected pressure field we can obtain the plane wave reflection coefficients as a function of the horizontal wave number or equivalently as a function of the angle of incidence. Suitable methods have been developed for performing the Hankel transform operation[14-18]. Schoeberg[19] uses a different approach to obtain the plane-wave reflection coefficient from a measurement of the pressure field.

\subsubsection{Determination of sound speed and density profiles(step 2)}

In this step the sound speed and density profiles are obtained using the planewave reflection coefficients as input data. Direct inverse methods[20-22] can be used for this purpose.

\subsubsection{Determination of attenuation coefficient(step 3)}

Using the information on sound speed and density values obtained in the last step and the information on the plane wave reflection coefficient, the attenuation coefficient profile is obtained. In this dissertation a perturbation approach is used to accomplish this.

This method of determining the attenuation coefficient profile from planewave reflection coefficient obtained with a $\mathrm{CW}$ source experiment has the following advantages over the other methods where a broadband source is used. 
1. Since a C.W. source is used the effect of dispersion is not relevant. The sound speed obtained in step 2 of the scheme is the value at the frequency of the source.

2. No a priori assumption is made on the frequency dependence of attenuation. The scheme provides the attenuation as a function of depth at the operating frequency

3. By doing simultaneous inversion for attenuation coefficient and corrections to sound speed and density structure, the effects of errors in sound speed and density on the attenuation profile can be overcome.

\subsection{Statement of the problem for the determination of attenuation coefficient}

For the purpose of this dissertation we will assume that by some suitable experiment and subsequent processing the plane wave reflection coefficient as a function of angle of incidence has been obtained. Further, we assume that this information has been used in an inverse scheme to obtain a close estimate of both compressional wave speed and the density profile for the bottom.

Consider the two models of the ocean bottom in Figure 3-7. The model on the top is the true model of the ocean bottom with density, sound speed and attenuation profiles as indicated. The other model is our present knowledge of the parameters describing the ocean bottom(i.e) a close estimate of sound speed and density stucture as obtained in the earlier stage of processing. The problem is to develop a method to determine the attenuation profile and corrections to the sound speed structure and density structure for the sediment layer. In solving the problem the following assumptions will be made.

1. The ocean bottom is horizontally stratified. 
$-72-$

\begin{tabular}{|c|c|c|c|}
\hline $\begin{array}{l}\text { Sound Speed } \\
\text { Density }\end{array}$ & $\begin{array}{l}=C_{0} \\
=\rho_{0}\end{array}$ & $\begin{array}{c}\text { Water Column } \\
- \\
\end{array}$ & \\
\hline Sound Speed & $=C(z)$ & \multirow{3}{*}{$\begin{array}{l}\text { Sediment } \\
\text { Loyer }\end{array}$} & rpm- \\
\hline $\left.\begin{array}{l}\text { Attenuation } \\
\text { Coefficent }\end{array}\right\}$ & $=a(z)$ & & \\
\hline Density & $=P(z)$. & & \\
\hline Sound Speed & $=c_{2}$ & Sub-Bottom & \\
\hline $\left.\begin{array}{l}\text { Attenuation } \\
\text { Coefficent }\end{array}\right\}$ & $=a_{2}$ & \multirow[t]{2}{*}{$\cdot$} & \\
\hline Density & $=P_{2}$ & & \\
\hline \multicolumn{3}{|c|}{ EXACT OCEAN BOTTOM MODEL } & \\
\hline
\end{tabular}

\begin{tabular}{|c|c|c|}
\hline $\begin{array}{l}\text { Sound Speed } \\
\text { Density }\end{array}$ & $\begin{array}{l}=C_{0} \\
=P_{0}\end{array}$ & Water Column \\
\hline Sound Speed & $=C_{b}(z)$ & \\
\hline $\left.\begin{array}{l}\text { Attenuation } \\
\text { Coefficent }\end{array}\right\}$ & $=0$ & $\begin{array}{l}\text { Sediment } \\
\text { Layer }\end{array}$ \\
\hline Density & $=p_{b}(z)$ & \\
\hline Sound Speed & $=C_{2}$ & Sub-Botiom \\
\hline $\left.\begin{array}{l}\text { Attenuotion } \\
\text { Coefficent }\end{array}\right\}$ & $=a_{2}$ & \\
\hline Densiły & $=P_{2}$ & \\
\hline
\end{tabular}

INITIAL GUESS OF OCEAN BOTTOM MODEL

Figure 3-7: Exact and guess model for the ocean bottom 
2. Circular symmetry exists in the point source field.

3. The sediments can be modelled as a fluid.

To simplify the analysis, we further assume that the acoustic parameters are known in the region $z<0$ and $z>h$ and that the parameters need be determined only in the region $0<z<h$.

Ve start by assuming that there are no errors in the sound speed and density and only the attenuation profile needs to be determined. Then, treating errors in the sound speed and density as unknowns, the problem is extended to the determination of these as well. 


\subsection{References}

1. L.D. Hampton,"Acoustic Properties of Sediments", J. Acoust. Soc. Am., 42, 882-880 (1967).

2. E.C. Mcleroy and A. Delvach, "Sound speed and attenuation from 15 to $1500 \mathrm{kHz}$ measures in natur.: sea-floor sediments," J. Acoust. Soc. Am., 44, 1148-1150 (1968).

3. A.W. Nolle, W.A. Hoger, J.F. Misfud, W.R. Runyan and M.B. Ward, "Acoustic properties of water nilled sediments," J. Accust. Soc. Am., 35, 1384-1408 (1963).

4. E.L. Hamilton, "Compressional wave attenuation in marine sediments, "Geophysics, 37, 620-646 (1972).

5. G. Shumway, "Sound speed and absorption studies of marine sediments by a resonance method," Geophysics, 25, 451-467, 659-685 (1970).

6. G.V. Frisk , J.A. Doutt, E.E. Hays, "Bottom interactions of low frequency acoustic signals at small grazing angles in the deep ocean," J. Acoust. Soc. Am., 69, 84-84 (1981).

7. R.S. Anderson and A. Blackman, "Attenuation of low frequency sound waves in sediments, "J. Acoust. soc. Am., 49, 786-791 (1971).

8. R.S. Jacobson, G.G.Shor,Jr., L.M.Dorman, "Linear inversion of body wave data-part II: Attenuation versus depth using spectral ratios," Geophysics, 46, 152-162 (1981).

9. K. Wrolstad, "Interval velocity and attenuation measurements in sediments from marine seismic reflection data, "J. Acoust. Soc. Am.,68(5), 1415-1435 (1980).

10. P.S. Hauge, "Measurements of attenuations from vertical seismic profiles," Geophysics, 46, 1548-1558 (1981).

11. S.K. Mitchell and K.C. Focke, "New measurements of compressional wave attenuation in deep ocean sediments," J. Acoust. Soc. Am.,67(5), 1582-1589 (1980). 
12. R.D. Stoll and R.E. Houtz, "Attenuation measurements from sonobuoys," J. Acoust. Soc. Am.,73(11), 163-172 (1983).

13. C.N. Spofford, "Inference of geo-acoustic parameters from bottom loss data," in Bottom-interacting ocean acoustics,(Plenum, New York,1980).

14. D.R. Mook, G.V. Frisk, and A.V. Oppenheim,"A hybrid numerical/analytic technique for the computation of wave fields in stratified media based on the Hankel transform,"J. Acoust. Soc. Am., 76(1), 222-244 (1984).

15. G.V. Frisk, A.V. Oppenheim, and D. Martinez, "A technique for measuring the plane wave reflection coefficient of ocean bottom," J. Acoust. Soc. Am., 68, 602-612 (1980).

16. D.R. Mook, "The numerical synthesis and inversion of Acoustic fields using Hankel transform with application to the estimation of the plane wave reflection coefficients," Sc.D. thesis,M.I.T./W.H.O.I Joint Program, Cambridge ,MA., and Woods Hole, MA. (January 1983).

17. G.V. Frisk, D.R. Mook, J.A. Doutt, E.E. Hays, and A.V. Oppenheim, "The application to real data of a technique for measuring the planewave reflection coefficient of the ocean bottom," J. Acoust. Soc. Am., 72(s1) S97(a), (1982).

18. A.V. Oppenheim, G.V. Frisk, and D.R. Martinez, "Computation of the Hankel transform using projectors," J. Acoust. Soc. Am.,68(2), 523-529 (1980).

19. M. Schoenberg, "Non-parametric estimation of the ocean bottom reflection coefficient," J. Acoust. Soc. Am., 64(4), 1165-1170 (1978).

20. I.M. Gel'fand and B.M. Levitan, "On the determination of a differential equation from its spectral function, "Isvestiya Akad. Nauk S.S.S.R. 15, 309-360 (1951); Am. Math. Soc. Transl. (1), 253-304 (1955).

21. H.E. Moses and C.M. Ridder, "Properties of dielectrics from reflection coefificients in one dimension," MIT Lincoln Lab.Tech. Report No. 322,1-47 (1963).

22. D.C. Stickler, "Inverse scattering in a stratified medium," J. Acoust. Soc. Am., 74,994-1005 (1983). 


\section{Chapter 4 The Forward Problem}

The forward problem deals with the determination of the response of a system to a known input given system parameters. In the context of our problem, it deals with the determination of the acoustic field in the sediment layers for a plane wave of unit amplitude incident on the top of the sediment. The acoustic characteristics of the sediment layer and the sub-bottom are assumed known. We need to solve the forward problem for two reasons. First, the plane wave reflection coefficient and the pressure field in the sediment layers for the assumed guess model are needed to solve the inverse problem as we will see in the next chapter. Second, we use synthetic data to test the inversion scheme and for this purpose the plane wave reflection coefficient is required to be computed. Two methods for solving the forward problem are presented in this chapter; a uniform asymptotic method and a method based on a propagator matrix formulation.

We use the ocean bottom model developed in Chapter 2 and represented in Figure 4-1. Consider a plane wave of unit amplitude incident at the water sediment interface inclined at an angle $\theta$ to the vertical. The acoustic field in the ocean and the bottom obey the acoustic wave equation. If $\phi$ represents the pressure field; the wave equation for $\phi$ is

$$
\frac{\partial^{2} \phi}{\partial x^{2}}+\frac{\partial^{2} \phi}{\partial z^{2}}+\rho(z)\left(\frac{1}{\rho(z)}\right)^{\prime} \frac{\partial \phi}{\partial z}+k^{2}(z) \phi=0
$$

Writing $\phi(x, z)=P(z) e^{i k} x^{x}$ the wave equation becomes 
$-77-$

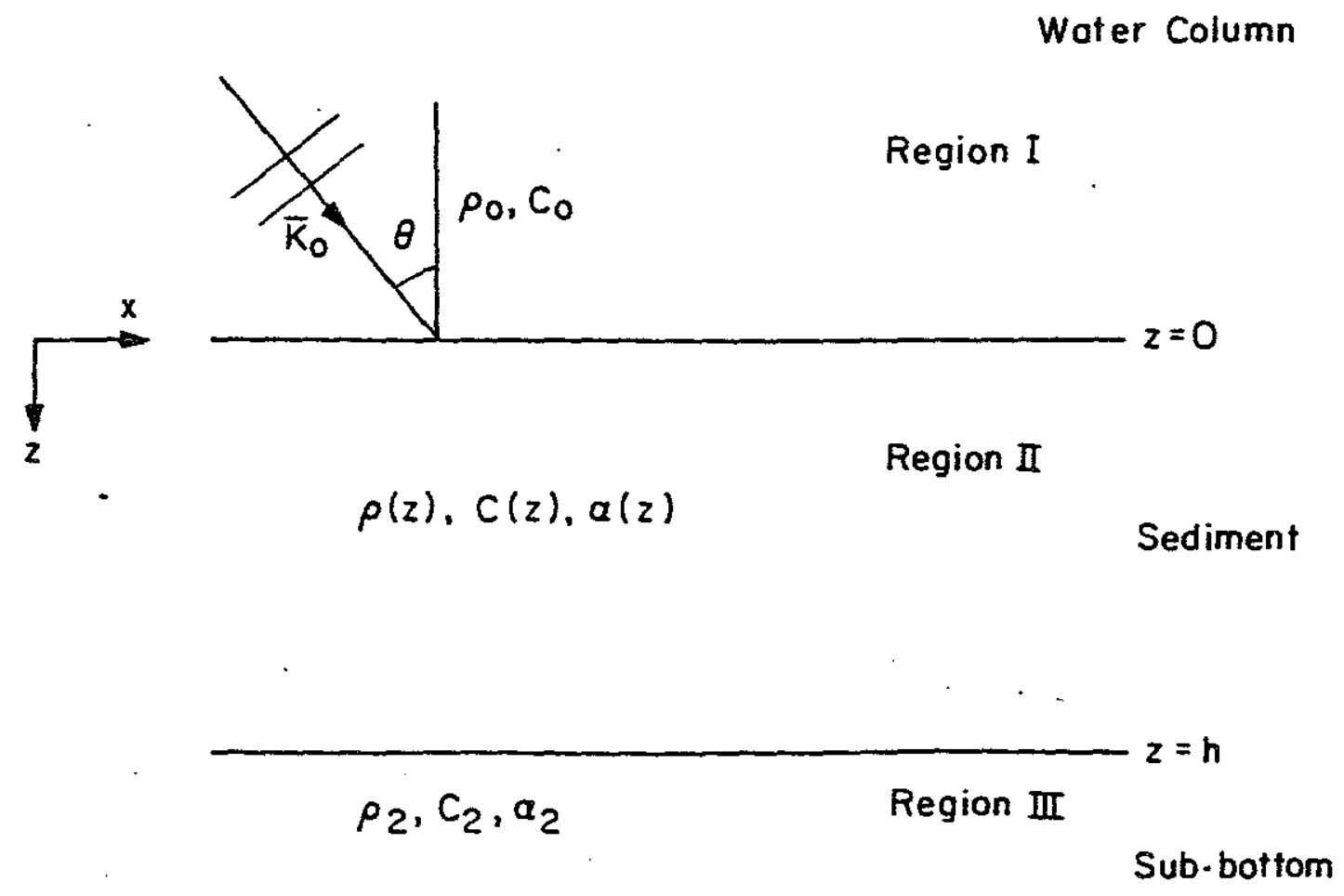

Figure 4-1: Ocean bottom model 


$$
\frac{d^{2} P(z)}{d z^{2}}+\rho(z)\left(\frac{1}{\rho(z)}\right)^{\prime} \frac{d P(z)}{d z}+\left(k^{2}(z)-k_{x}^{2}\right) P(z)=0 .
$$

Defining a new variable $\mathrm{v}(\mathrm{z})$ as $v(z)=\rho^{-1 / 2}(z) P(z)$ and substituting in the wave equation we obtain the following relation.

$$
\frac{d^{2} v(z)}{d z^{2}}+Q(z) v(z)=0
$$

where,

$$
Q(z)=k^{2}(z)-k_{x}{ }^{2}+\frac{\rho^{1 / 2}(z)}{2}\left(\frac{\rho^{\prime}(z)}{\rho^{3 / 2}(z)}\right)^{\prime}
$$

When the density in the medium is a constant the wave equation takes the form given below.

$$
\frac{d^{2} P(z)}{d z^{2}}+\left(k^{2}(z)-k_{x}^{2}\right) P(z)=0
$$

The wave equation in the three regions will then be as given below.

Water column (Region I):

$$
\frac{d^{2} P_{0}}{d z^{2}}+\left(k_{0}^{2}-k_{x}^{2}\right) P_{0}=0
$$

where $k_{0}$ is the wave number in region I (i.e.) $k_{0}=\omega / C_{0}$.

Sediment layers (Region II):

$$
\frac{d^{2} v}{d z^{2}}+Q(z) v(z)=0
$$

Sub-bottom (Region III): 


$$
\frac{d^{2} P_{2}}{d z^{2}}+\left(k_{2}{ }^{2}-k_{x}{ }^{2}\right) P_{2}=0
$$

Where $k_{2}$ is the wave number in region III (i.e.) $k_{2}=\omega / C_{2}$.

For an incident wave of unit amplitude, the field in region $I$ is the sum of the incident and reflected waves.

$$
P_{0}=e^{i k} z 0^{z}+R\left(k_{x}\right) e^{-i k} z 0^{z}
$$

In the above equation $R\left(k_{x}\right)$ is the plane wave reflection coefficient of the entire bottom structure and $k_{z 0}=\left(k_{0}{ }^{2}-k_{x}{ }^{2}\right)^{1 / 2}$ is the vertical wave number in the water column. The subbottom is an infinite half space and only outward propagating wave exists in this region. The field in the subbottom is, therefore, given by the following expression.

$$
P_{2}=T\left(k_{x}\right) e^{i k_{z 2} z}
$$

where $k_{z 2}$ is the vertical wave number in the subbottom and $T\left(k_{x}\right)$ is the transmission coefficient. Note that $k_{z 2}$ is complex to allow for attenuation in the bottom. The pressure field in the sediment layer is obtained by solving equation (4.7). The boundary conditions that the solution has to satisfy are the continuity of pressure and normal particle velocity at the two interfaces i.e at $z=0$ and $z=h$ representing the water-sediment and sediment-subbottom interfaces. The application of these conditions at the sediment-water interface leads to the following equations.

$$
P(0)=1+R
$$




$$
\frac{1}{\rho(0)} P^{\prime}(0)=\frac{i k_{z 0}}{\rho_{0}}(1-R)
$$

In the above equations $\rho(0)$ is the density in the sediment at the interface while $\rho_{0}$ is the density in the water column. $P(0)$ is the pressure field in the sediment at $z=0$. The primes denote derivatives with respect to $z$. Similarly applying the boundary conditions at the other interface yields a second set of equations.

$$
\begin{aligned}
& P(h)=T e^{i k_{z 2} h} \\
& \frac{1}{\rho(h)} P^{\prime}(h)=\frac{i k_{z 2} T}{\rho_{2}} e^{i k_{z 2} h}
\end{aligned}
$$

\subsection{Uniform asymptotic solution}

The uniform asymptotic solution[1] to the wave equation in region $\mathbb{1}$ has been discussed in detail by Kawahara[2]. The solution that is valid when the function $Q(z)$ varies smoothly and there is only one turning point, is

$$
v(z)=c_{1} Q^{-1 / 4}(z) \gamma^{1 / 4} A_{i}(\gamma(z))+c_{2} Q^{-1 / 4}(z) \gamma^{1 / 4} B_{i}(\gamma(z))
$$

where $A_{i}$ and $B_{i}$ are the Airy functions. $\gamma(z)$ is given by the expression

$$
\gamma(z)=3 / 2\left(\int_{z_{t}}^{z} Q^{1 / 2}(\xi) d \xi\right)^{2 / 3}
$$

and $z_{t}$ corresponds to the turning point i.e $Q\left(z_{t}\right)=0$. The solution for $P(z)=\rho^{1 / 2}(z) v(z)$ is then;

$$
P(z)=c_{1} g_{1}(z)+c_{2} g_{2}(z)
$$


where

$$
\begin{aligned}
& g_{1}(z)=c_{1} \rho^{1 / 2}(z) Q^{-1 / 4}(z) \gamma^{1 / 4} A_{i}(\gamma(z)) \\
& g_{2}(z)=c_{2} \rho^{1 / 2}(z) Q^{-1 / 4}(z) \gamma^{1 / 4} B_{i}(\gamma(z))
\end{aligned}
$$

and $c_{1}$ and $c_{2}$ are arbitrary constants.

Applying the boundary conditions at the two interfaces we now obtain a set of four equations with four unknowns to be determined namely the cofficients $c_{1}$ and $c_{2}$ and the reflection and transmission coefficients $R$ and $T$. Solving for these unknowns, we obtain $R, T, c_{1} a n d c_{2}$. The coefficients are now substituted in equation (4.17) and the field obtained. Kawahara[2] has shown that for certain canonical

profiles like $c$ linear, $c^{2}$ linear and $c^{3}$ linear the integral in (4.16) can be performed analytically.

\subsection{Propagator matrix method}

The second method for solving the forward problem models the sediment as a stack of homogeneous layers. By making the thickness of the layers small any arbitrary variation of density, sound speed and attenuation can be accommodated. Mook[3] has proposed a method for determining the reflection coefficient using the propagator martix approach. We will essentially follow his approach and indicate how a numerically stable algorithm is obtained for computing the plane wave reflection coefficients and the field in the sediment layer.

The propagator matrix method is dealt with extensively in the literature[4-6]. We will explain in brief the essential features of the propagator matrix method before taking up the problem indicated above. 
$-82-$

Any second order differential equation can be written as a set of first order equations. The general representation is given below.

$$
\frac{d \mathbf{f}}{d z}-\mathbf{A}(z) \mathbf{f}(z)=0
$$

For the equation (4.2), $f$ is an $2 \times 1$ vector and $A$ is a $2 \times 2$ matrix. The propagator matrix (also called matrizant) is defined as

$$
\begin{aligned}
\mathbf{P}\left(z, z_{0}\right)= & \mathbf{I}+\int_{z_{0}}^{z} \mathbf{A}\left(\xi_{1}\right) d \xi_{1}+ \\
& \int_{z\left({ }_{0}\right)}^{z} \mathbf{A}\left(\xi_{1}\right) \int_{z_{0}}^{\xi_{1}} d \xi_{1} d \xi_{2}+\cdots+
\end{aligned}
$$

where $I$ is the identity matrix of the order of $\mathbf{A}$. By substitution it can be shown that $\mathrm{P}\left(z, z_{0}\right)$ satisfies the following differential equation

$$
\frac{d^{2} \mathbf{P}\left(z, z_{0}\right)}{d z^{2}}-\mathbf{A}(z) \mathbf{P}\left(z, z_{0}\right)=0
$$

Also $\mathrm{P}\left(z_{0}, z_{0}\right)=\mathbf{I}$. The most important property of the propagator matrix is that it enables us to obtain $\mathbf{f}(z)$ from its value at some other point $z_{0}$.

$$
\mathbf{f}(z)=\mathbf{P}\left(z, z_{0}\right) \mathbf{f}\left(z_{0}\right)
$$

To see that this is true,we substitute $f(z)$ in the differential equation. Then,

$$
\frac{d^{2} \mathbf{f}(z)}{d z^{2}}-\mathbf{A}(z) \mathbf{f}(z)=0
$$

Therefore, $f(z)$ satisfies the differential equation. Also it gives the value of $\mathbf{f}(z)$ at $z_{0}$ correctly. 


$$
\mathbf{f}\left(z_{0}\right)=\mathbf{P}\left(z_{0}, z_{0}\right) \mathbf{f}\left(z_{0}\right)=\mathbf{f}\left(z_{0}\right)
$$

Another interesting property of the propagator matrix is shown by the following equations.

$$
\begin{aligned}
\mathbf{f}\left(z_{2}\right) & =\mathbf{P}\left(z_{2}, z_{1}\right) \mathbf{f}\left(z_{1}\right) \\
& =\mathbf{P}\left(z_{2}, z_{1}\right) \mathbf{P}\left(z_{1}, z_{0}\right) \mathbf{f}\left(z_{0}\right)
\end{aligned}
$$

If we now choose $z_{2}=z_{0}$ then,

$$
\mathbf{f}\left(z_{0}\right)=\mathbf{P}\left(z_{0}, z_{1}\right) \mathbf{P}\left(z_{1}, z_{0}\right) \mathbf{f}\left(z_{0}\right)
$$

or

$$
\mathbb{1}=\mathbf{P}\left(z_{0}, z_{1}\right) \mathbf{P}\left(z_{1}, z_{0}\right)
$$

When the matrix $\mathbf{A}$ is not dependent on $z$ as in the case of homogeneous layers,

$$
\begin{aligned}
& \mathrm{P}\left(z, z_{0}\right)=\mathbf{I}+\left(z-z_{0}\right) \mathrm{A}+1 / 2\left(z-z_{0}\right)^{2} \mathrm{~A}^{2}+\cdots+ \\
& \mathrm{P}\left(z, z_{0}\right)=e^{\left(z-z_{0}\right) \mathbf{A}}
\end{aligned}
$$

For a square matrix $\mathbf{A}$ with distinct eigenvalues, $\lambda_{k}(k=1, \cdots, n)$ by the Sylvester theorem,

$$
F(\mathbf{A})=\sum_{k=1}^{n} F\left(\lambda_{k}\right) \prod_{r \neq k} \frac{\mathbf{A}-\lambda_{k} \mathbf{I}}{\lambda_{k}-\lambda_{r}}
$$

Reverting now to the differential equation (4.2) it is written as a set of first order equations. 
$-84-$

$$
\begin{aligned}
& P(0), W(0)
\end{aligned}
$$

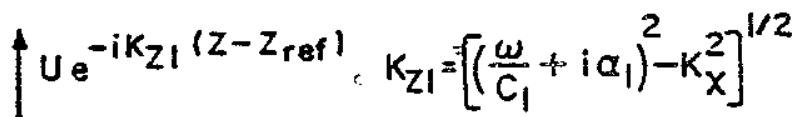

$$
\begin{aligned}
& D e^{i k_{z 1}\left(z-z_{\text {ref }}\right)}
\end{aligned}
$$

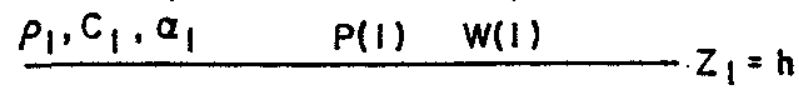

Figure 4-2: Single layer model 


$$
\frac{d}{d z}\left[\begin{array}{l}
P \\
w
\end{array}\right]=\mathrm{i}\left[\begin{array}{ll}
0 & \omega \rho \\
\frac{k_{z}^{2}}{\omega \rho} & 0
\end{array}\right]\left[\begin{array}{l}
P \\
w
\end{array}\right]
$$

When applied to a stack of homogeneous layers $\rho(\mathrm{z})$ and $k_{z}$ represent the density and the vertical wave number in the layer under consideration. As before $\omega$ represents the frequency and the vector $\mathbf{f}$ contains the elements $P$ and $w$, the pressure and normal particle velocity. The matrix $\mathbf{A}$ for the above equation is, therefore

$$
\mathrm{A} \quad-\mathrm{i}\left[\begin{array}{ll}
0 & \omega \rho(\mathrm{z}) \\
\frac{k_{z}^{2}}{\omega \rho(\mathrm{z})} & 0
\end{array}\right]
$$

The propagator matrix is now obtained using equation (4.29).

$$
P\left(z-z_{0}\right)=\left[\begin{array}{ll}
\cos \left(k_{z}\left(z-z_{0}\right)\right) & \frac{\mathrm{i} \omega \rho}{k_{z}} \sin \left(z-z_{0}\right) \\
\mathrm{i}-k_{z} \sin \left(z-z_{0}\right) & \cos \left(z-z_{0}\right)
\end{array}\right]
$$

In the above, $Y=\frac{k_{z}}{\omega \rho}$ is the admittance.

Consider a layer of thickness $h$ as shown in figure 4-2. The pressure and particle velocity at depth $z=0$ is related to the pressure and particle velocity at depth $z=h$ by the following equation.

$$
\left[\begin{array}{l}
P(1) \\
w(1)
\end{array}\right]=\left[\begin{array}{ll}
\cos \left(k_{z 1} h\right) & \frac{\mathrm{i}}{Y(1)} \sin \left(k_{z 1} h\right) \\
\mathrm{i} Y(1) \sin \left(k_{z 1} h\right) & \cos \left(k_{z 1} h\right)
\end{array}\right]\left[\begin{array}{l}
P(0) \\
w(0)
\end{array}\right]
$$

Since each layer is homogeneous, the pressure and normal particle velocity in the layers can be written as a sum of up and down going waves. 
$-86-$
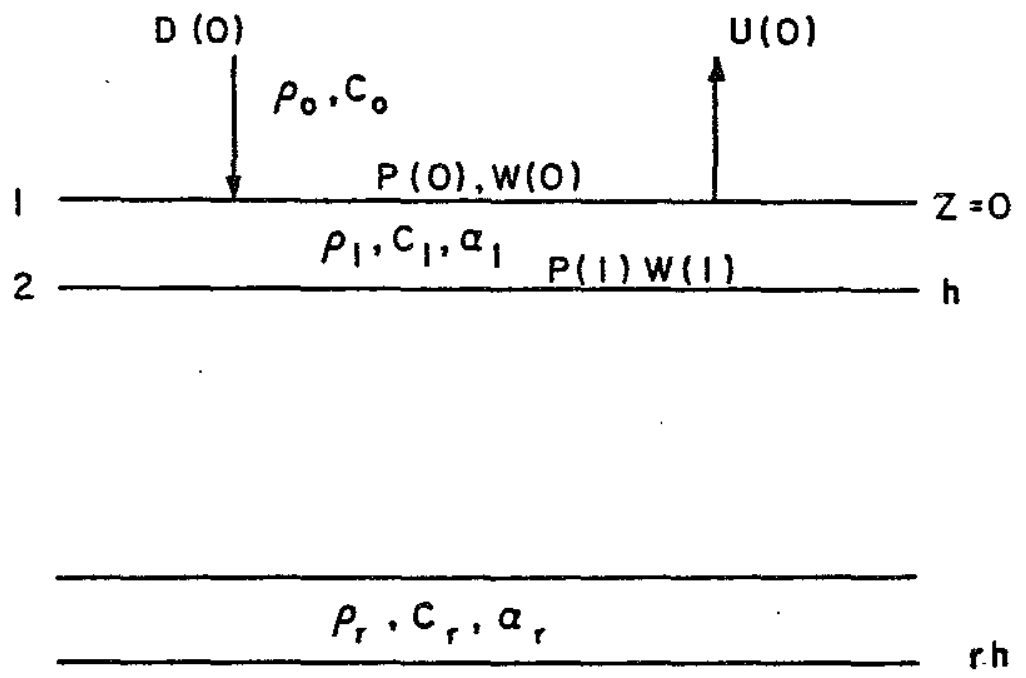

$\frac{P(n-2), W(n-2)}{\frac{\rho_{n-1}, C_{n-1}, a_{n-1} \quad P(n-1), W(n-1)}{P_{n} \mid \begin{array}{l}C_{n,}, a_{n} \\ D(n)\end{array}}} \quad z=(n-1) h$

Figure 4-3: Multi-layer model 


$$
\begin{aligned}
& P(z)=D e^{\left.i k_{z 1} \mid z-z_{r e f}\right\rfloor}+U e^{\left.-i k_{z 1} \mid z-z_{r e f}\right\rfloor} \\
& w(z)=\frac{k_{z 1}}{\omega \rho}\left(D e^{i k_{z 1}[z-z} r e d-U e^{-i k_{z 1}[z-z} r e f\right)
\end{aligned}
$$

or,

$$
\left[\begin{array}{l}
P(z) \\
w(z)
\end{array}\right]=\left[\begin{array}{ll}
1 & 1 \\
\frac{k_{z 1}}{\omega \rho} & \frac{-k_{z 1}}{\omega \rho}
\end{array}\right]\left[\begin{array}{ll}
\mathrm{e}^{\mathrm{i} k_{z 1}\left(z-z_{r e \rho} d\right.} & 0 \\
0 & \mathrm{e}^{-\mathrm{i} k_{z 1}\left(z-z_{r e f} d\right.}
\end{array}\right]\left[\begin{array}{l}
D \\
U
\end{array}\right]
$$

Setting $z_{r e f}=\mathbf{0}$, we obtain

$$
\left[\begin{array}{c}
P(z) \\
w(z)
\end{array}\right]=\left[\begin{array}{ll}
\mathrm{e}^{\mathrm{i} k} 1^{z} & \mathrm{e}^{-\mathrm{i} k} z 1^{z} \\
Y(1) \mathrm{e}^{\mathrm{i} k} z 1^{z} & -Y(1) \mathrm{e}^{-\mathrm{i} k_{z 1} z}
\end{array}\right]\left[\begin{array}{l}
D \\
U
\end{array}\right]
$$

where,

$$
Y(1)=\frac{k_{z 1}}{\omega \rho}
$$

Now consider a stack of homogeneous layers as shown in Figure 4-3. Then the pressure and particle velocity at $z=0$ is related to the up and down going waves in the watercolumn by the following equation.

$$
\left[\begin{array}{l}
P(0) \\
w(0)
\end{array}\right]=\left[\begin{array}{ll}
1 & 1 \\
Y(0) & -Y(0)
\end{array}\right]\left[\begin{array}{l}
D(0) \\
U(0)
\end{array}\right]
$$

or equivalently, 


$$
\left[\begin{array}{l}
D(0) \\
U(0)
\end{array}\right]=\frac{1}{2} \quad\left[\begin{array}{cc}
1 & 1 \\
1 & -1
\end{array}\right]\left[\begin{array}{l}
P(0) \\
\frac{w(0)}{Y(0)}
\end{array}\right]
$$

Following the method of Mook[3] the matrix in equation (4.33) is modified and the elements expressed in terms of ratio of impedances. This is done to avoid difficulties that may arise due to $\mathrm{P}$ and $\mathrm{w}$ being of different scales of magnitude.

$$
\left[\begin{array}{l}
P(0) \\
\zeta(0) w(0)
\end{array}\right]=\left[\begin{array}{ll}
\cos \left(k_{z}(1) h\right) & -\operatorname{isin}\left(k_{z}(1) h\right) \\
-\mathrm{i} \xi(1) \sin \left(k_{z}(1) h\right) \xi(1) \cos \left(k_{z}(1) h\right)
\end{array}\right]\left[\begin{array}{l}
P(1) \\
\zeta \\
\zeta(1) w(1)
\end{array}\right]
$$

where $\xi(1)=Y(1) / Y(0), \zeta(0)=1 / Y(0)$, and $g(z)(1)=1 / Y(1)$. Using the propagator matrix we can relate the $P$ and $w$ at the nth interface to the values of $P$ and $w$ at the first interface:

$$
\left[\begin{array}{l}
P(0) \\
\zeta(0) w(0)
\end{array}\right]=\left(\phi_{1} \ldots \phi_{\mathrm{n}-1}\right)\left[\begin{array}{l}
P(\mathrm{n}-1) \\
\zeta(\mathrm{n}-1) w(\mathrm{n}-1)
\end{array}\right]
$$

where $\phi \mathrm{s}$ are the propagator matrices for the layers from 1 to $\mathrm{n}-1$. The pressure and particle velocity at the bottommost interface is now related to the up and down going waves in the terminating half space.

$$
\left[\begin{array}{l}
P(\mathrm{n}-1) \\
\zeta(\mathrm{n}-1) w(\mathrm{n}-1)
\end{array}\right]=\left[\begin{array}{ll}
1 & 1 \\
\xi(\mathrm{n}) & -\xi(\mathrm{n})
\end{array}\right]\left[\begin{array}{l}
D(\mathrm{n}) \\
U(\mathrm{n})
\end{array}\right]
$$

The relation between $D(0), U(0)$ and $D(n), U(n)$ can now be obtained.

$$
\left[\begin{array}{l}
D(0) \\
U(0)
\end{array}\right]=\frac{1}{2}\left[\begin{array}{cc}
1 & 1 \\
1 & -1
\end{array}\right]\left(\phi_{1} \ldots . \phi_{\mathrm{n}-1}\right) \quad\left[\begin{array}{cc}
1 & 1 \\
\xi(\mathrm{n})-\xi(\mathrm{n})
\end{array}\right]\left[\begin{array}{l}
D(\mathrm{n}) \\
U(\mathrm{n})
\end{array}\right]
$$


or,

$$
\left[\begin{array}{l}
D(\mathbf{0}) \\
U(\mathbf{0})
\end{array}\right]=\boldsymbol{\Phi}\left[\begin{array}{l}
D(\mathbf{n})+U(\mathbf{n}) \\
s(\mathbf{n})\{D(\mathbf{n})-U(\mathbf{n})\}
\end{array}\right]
$$

where

$$
\Phi=\frac{1}{2}\left[\begin{array}{cc}
1 & 1 \\
1 & -1
\end{array}\right] \quad\left(\phi_{1} \ldots \phi_{\mathrm{n}-1}\right)
$$

and $\phi$ is a $2 \times 2$ matrix with elements $\phi_{11}, \phi_{12}, \phi_{21}$ and $\phi_{22}$. In the infinite half space representing the subbottom $\mathrm{U}(\mathbf{n})=0$. Therefore,

$$
\left[\begin{array}{l}
D(\mathbf{0}) \\
U(\mathbf{0})
\end{array}\right]=\boldsymbol{\Phi} \quad\left[\begin{array}{l}
D(\mathbf{n}) \\
\xi(\mathbf{n}) D(\mathbf{n})
\end{array}\right]
$$

The reflection coefficient is then given by

$$
R=\frac{U(0)}{D(0)}=\frac{\phi_{21}+\xi(n) \phi_{22}}{\phi_{11}+\xi(n) \phi_{12}}
$$

Having obtained the reflection coefficient the pressure and particle velocity at the top interface are easily found to be $P(0)=1+R$ and $w(0)=Y(0)(1-R)$ respectively. We can now propagate the pressure and particle velocity downward and determine it at any layer. This is similar to solving the differential equation given its initial conditions. If this procedure is adopted, the scheme becomes numerically unstable and the solution rapidly diverges from the correct solution in regions where the waves become inhomogeneous. Hawker and Foreman[7] give examples to show that the direction in which the integration is carried out is important to obtain a stable solution and recommend that the integration be done 
upwards starting with the conditions at the bottommost interface as the initial conditions.

To do this, we assume that $D(n)=1.0$ and $U(n)=0$. The pressure and particle velocity at the $n$th interface is then related to $D(n)$ and $U(n)$.

$$
\left[\begin{array}{l}
P(\mathrm{n}-1) \\
\zeta(\mathrm{n}-1) w(\mathrm{n})
\end{array}\right]=\left[\begin{array}{ll}
1 & 1 \\
\xi(\mathrm{n}) & -\xi(\mathrm{n})
\end{array}\right]\left[\begin{array}{l}
1 \\
0
\end{array}\right]
$$

We now relate $P(\mathrm{n}-1)$ and $w(\mathrm{n}-1)$ to $P(\mathrm{n}-2)$ and $w(\mathrm{n}-2)$.

$$
\left[\begin{array}{l}
P(\mathrm{n}-2) \\
s(\mathrm{n}-2) w(\mathrm{n}-2)
\end{array}\right]=\phi\left[\begin{array}{l}
P(\mathrm{n}-1) \\
s(\mathrm{n}-1) w(\mathrm{n}-1)
\end{array}\right]
$$

As before relating the up and down going waves at the top to the up and down going waves in the infinite halfspace in the bottom, we obtain the following expression.

$$
\left[\begin{array}{l}
D(0) \\
U(0)
\end{array}\right]=\Phi\left[\begin{array}{l}
1 \\
\xi(\mathbf{n})
\end{array}\right]
$$

This gives the reflection coefficient as

$$
R=\frac{U(0)}{D(0)}=\frac{\phi_{21}+\xi(n) \phi_{22}}{\phi_{11}+\xi(n) \phi_{12}}
$$

The pressure computed will correspond to an incident amplitude of $D(0)$. The pressure field at any point in the layers is then obtained by dividing through by $D(0)$. In this thesis we use the propagator matrix method to compuie the reflection coefficient and the pressure field in the sediment layers. 


\subsection{References}

1. C.M. Bender and S.A. Orszag, Advanced mathematical methods for scientists and engineers, (McGraw-Hill, New York, 1978).

2. H. Kawahara, "Asymptotic analysis of ocean bottom reflected acoustic fields," Sc.D Thesis,M.I.T./W.H.O.I. Joint Program, Cambridge,MA. and Woods Hole, MA. (May, 1984).

3. D.R. Mook, "The numerical synthesis and inversion of acoustic fields using the Hankel transform with application to the estimation of the plane wave reflection coefficent of the ocean bottom, "Sc.D. Thesis, M.I.T./W.H.O.I. Joint Program, Cambridge, MA., and Woods Hole, MA. (January, 1983).

4. W.T. Thompson, "Transmission of elastic waves through a stratified solid," Journal of Applied Physics, 21, 89-93, (1950).

5. N.A. Haskell, " The dispersion of surface waves in multilayered media," Bulletin of the Seismological Society of America, 43, 17-34 (1953).

6. Keiiti Aki, and P.G. Richards, Quantitative Seismology: Theory and Methods, Vol. 1, (Foreman, San Fracisco,1980).

7. K.E. Hawker, and T.L. Foreman, "A plane wave reflection loss model based on numerical integration," J. Acoust. Soc. Am., 64, 1470-1476 (1978). 


\section{Chapter 5 \\ Formulation of the Inverse Problem}

In Chapter 3 we proposed a sequence of steps for the determination of the acoustic parameters from the measured pressure field. We also gave a statement of the problem that is addressed in this thesis. In Chapter 4 we dealt with the solution of the forward problem. In this chapter we formulate the inverse problem, namely, the determination of the attenuation coefficient profile in the sediment layer using the plane wave reflection coefficient at various angles of incidence as input information. The real and imginary part of the wavenumber at $220 \mathrm{~Hz}$ are given in Table 5-I for different types of marine sediments. These have been obtained using data presented by Hamilton[1] for the compressional wave speed and attenuation coefficient. The attenuation coefficient at $220 \mathrm{~Hz}$ is computed by extrapolating into the low frequency region using a linear law. The attenuation coefficient is order of magnitude smaller than the real part of the wavenumber and can therefore, be treated as a perturbation of the real part. Hence, we formulate the inverse problem largely following the perturbation method introduced by Cohen and Bleistein[2]. They dealt with the problem of determining perturbations in sound speed strueture using the plane wave reflection coefficient at all frequencies and at a fixed angle of incidence as input information. In Chapter 3 we indicated the advantage of using a constant frequency source for determining the acoustic properties of marine sediments. Therefore, an essential difference between our formulation and theirs is that we use plane wave reflection coefficient at different angles of incidence and at a fixed frequency as input information. 


\begin{tabular}{|l|c|c|c|}
\hline $\begin{array}{c}\text { Type of } \\
\text { sediment }\end{array}$ & $\begin{array}{c}\text { Wave } \\
\text { speed } \\
\mathrm{m} / \mathrm{s}\end{array}$ & $\begin{array}{c}\text { Real } \\
\text { part of } \\
\text { wavenumber }\end{array}$ & $\begin{array}{c}\text { Imaginary } \\
\text { part of } \\
\text { wavenumber } \\
\text { nepers } / \mathrm{m}\end{array}$ \\
\hline Fine sand & 1750.0 & 0.79 & 0.0126 \\
\hline Silty sand & 1645.0 & 0.84 & 0.0145 \\
\hline Silty clay & 1520.0 & 0.91 & 0.0019 \\
\hline
\end{tabular}

Table 5-I: Real and imaginary parts of the wavenumber at $220 \mathrm{~Hz}$ for marine sediments 
In this chapter, we first arrive at a non-linear integral equation relating the plane wave reflection coefficient to the unknown attenuation coefficient profile. The integral equation is then linearised using the Born approximation. We also obtain the region of validity of the Born approximation.

Rytov approximation can also be used to obtain a similar linear integral equation. Keller[3] has shown that the Rytov approximation is valid for longer ranges than the Born approximation in regions where there is only one field, for example in regions where only transmitted field exists. Keller observes that where there are more than one field, the Rytov method has to be applied to each wave separately for it to retain its advantage over the the Born approximation. On the other hand, the Born approximation can be applied to the total field. In a recent work Oristaglio[4] studied the accuracy of the Born and Rytov approximation to the laws of reflection and refraction and noted that the Rytov method works reasonably well for in the backscattering regions as well even when the method is applied to the total field; a surprising result in the context of Kellers observations. We formulate the inverse problem using the Rytov method so as to make comparisons between the two methods.

Consider the two models in Figure 3-7. One of these is the true model and the other the guess model which we call the background model. The background model represents our present knowledge of the model. In this chapter we assume that the only unknown is the attenuation coefficient profile in the sediment layer. The other acoustic parameters of the two models are the same. We first determine the response at the water sediment interface of the two models to a plane wave of unit amplitude. For the true model this is obtained from a field experiment. For the background model we obtain this by solving the forward problem using the method described in Chapter 4 . We, then, relate the difference in response to the unknown 
$-95-$

attenuation coefficient profile and obtain an integral equation representation of the problem.

\subsection{Inverse problem formulation using Born approximation}

\subsubsection{Derivation of integral equation}

Consider a plane wave of unit amplitude incident at an angle $\theta$ as shown in figure 5-1. The pressure field in the sediment layer is given by the equation;

$$
\frac{\partial^{2} \phi}{\partial x^{2}}+\frac{\partial^{2} \phi}{\partial z^{2}}+\rho(z)\left(\frac{1}{\rho(z)}\right)^{\prime} \frac{\partial \phi}{\partial z}+k^{2}(z) \phi=0
$$

Let $\phi(x, z)=P(z) e^{i k} x^{x}$. Substituting in the equation (5.1) we obtain the following one dimensional wave equation for the field in the sediment.

$$
\frac{d^{2} P(z)}{d z^{2}}+\rho(z)\left(\frac{1}{\rho(z)}\right)^{\prime} \frac{d P(z)}{d z}+\left(k^{2}(z)-k_{x}^{2}\right) P(z)=0
$$

Let $v(z)=\rho^{-1 / 2}(z) P(z)$. Substituting this in equation (5.2), we obtain the Schrodinger type equation given below.

$$
\frac{d^{2} v(z)}{d z^{2}}+\left(k^{2}(z)+\mu(z)-k_{x}^{2}\right) v(z)=0
$$

where,

$$
\mu(z)=\frac{\rho^{1 / 2}(z)}{2}\left\{\frac{\rho^{\prime}(z)}{\rho^{3 / 2}(z)}\right\}^{\prime}
$$

We derive a similar equation for the backgroud model as well. The subscript ' $b$ ' in the following equations refer to the backgroud model. 


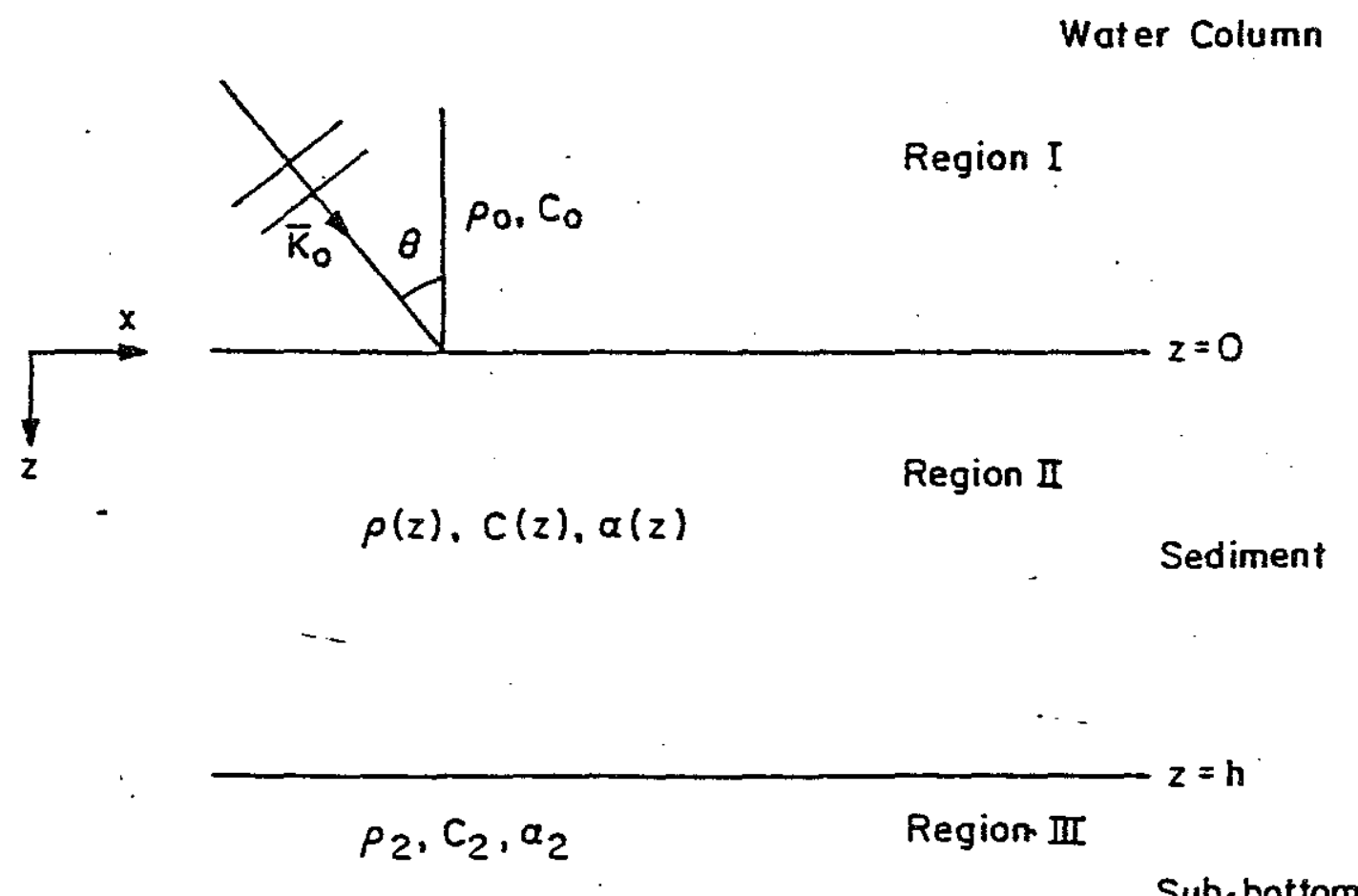

Figure 5-1: Ocean bottom model 


$$
\frac{d^{2} v_{b}(z)}{d z^{2}}+\left(k^{2}(z)+\mu_{b}(z)-k_{x}^{2}\right) v_{b}(z)=0
$$

where,

$$
\mu_{b}=\frac{\rho_{b}^{1 / 2}}{2}\left\{\frac{\rho_{b}^{\prime}(z)}{\rho_{b}^{3 / 2}(z)}\right\}^{\prime}
$$

The wavenumber in the sediment layer is a complex quantity and is given by the following expression.

$$
k(z)=\frac{\omega}{C(z)}+i \alpha(z)
$$

where $\alpha(z)$ is the unknown attenuation coefficient and $C(z)$ the sound speed profile. Since $C_{b}(z)=C(z)$ we write $k(z)=k_{b}(z)+i \alpha(z)$. We also note that $\mu(z)=\mu_{\mathrm{b}}(\mathrm{z})$. Substituting in the equation (5.3), we obtain

$$
\frac{d^{2} v(z)}{d z^{2}}+\left(k_{b}^{2}(z)+\mu_{b}(z)-k_{x}^{2}\right) v(z)=-2 i k_{b} \alpha(z) v(z) .
$$

Multiply equation (5.6) by $v_{b}(z)$ and equation (5.4) by $v(z)$ and subtract one from the other. We, then, obtain

$$
v_{b}(z) v^{\prime \prime}(z)-v(z) v_{b}^{\prime \prime}=-2 i k_{b}(z) \alpha(z) v(z) v_{b}(z)
$$

In the above equation the double prime denotes the second derivative with respect to $z$. Integrating both sides between the limits 0 to $h$ leads to the following equation.

$$
v_{b}(z) v^{\prime}(z)-\left.v_{b}^{\prime}(z) v(z)\right|_{0} ^{h}=\int_{0}^{h}-2 i k_{b}(z) v(z) v_{b}(z) \alpha(z) d z
$$


$-98-$

To determine $v(z), v_{b}(z), v^{\prime}(z), v_{b}^{\prime}(z)$, at the limits $z=0$ and $z=h$ we make use of the boundary conditions, i.e continuity of pressure and normal particle velocity. Since the water column is a homogeneous medium, the boundary conditions at the water sediment interface for the true model are given by the following expressions. $R$ is the plane wave reflection coefficient at the water sediment interface.

$$
P(0)=1+R, \quad P^{\prime}(0)=\frac{\rho_{b}(0)}{\rho_{0}} i k_{z 0}(1-R)
$$

A similar set of equations are obtained for the boundary condition at the other interface. Here $T$ is the transmission coefficient.

$$
P(h)=T e^{i k_{z 2} h}, P(h)=\frac{\rho_{b}(h)}{\rho_{2}} i k_{z 2} T e^{i k_{z 2} h}
$$

The boundary conditions for the background model obtained similarly are given below. $R_{b}$ and $T_{b}$ are the reflection and transmission coefficients for the background model.

$$
\begin{aligned}
& P_{b}(0)=1+R_{b}, \quad P_{b}^{\prime}(0)=\frac{\rho_{b}(0)}{\rho_{0}} i k_{z 0}\left(1-R_{b}\right) \\
& , P_{b}(h)=T_{b} e^{i k_{z 2} h}, P_{b}^{\prime}(h)=\frac{\rho_{b}(h)}{\rho_{2}} i k_{z 2} T_{b} e^{\left.i k_{z 2} h\right)}
\end{aligned}
$$

The left hand side of the equation $(5.8)$ is now evaluated using the relationship between $v(z), v_{b}(z)$ and $P(z)$ and $P_{b}(z)$ given above and the boundary conditions. After a some algebra we find the L.H.S. of equation reduces to zero at the upper limit while at the lower limit it is given by the following expression.

$$
\left.\left(v_{b}(z) v^{\prime}(z)-v(z) v_{b}^{\prime}(z)\right)\right|_{0}=\frac{2 i k_{z 0}}{\rho_{0}}\left(R_{b}-R\right)
$$


The integral equation is therefore,

$$
\frac{k_{z 0}\left(R_{b}-R\right)}{\rho_{0}}=\int_{0}^{h} k_{b}(z) \alpha(z) v(z) v_{b}(z) d z
$$

This is a non-linear integral equation and it is not possible to solve this equation for $\alpha(z)$ since $v(z)$ is dependent on the unknown $\alpha(z)$. As the attenuation is a small perturbation on the wavenumber we make the Born approximation $v(z)=v_{b}(z)$. The integral equation then, reduces to the following.

$$
\frac{k_{z 0}\left(R_{b}-R\right)}{\rho_{0}}=\int_{o}^{h k_{b}(z) \alpha(z)} \frac{\rho_{b}(z)}{\rho_{b}}(z) d z
$$

Since $k_{b}(z)$ and $\rho_{b}(z)$ are known, $P_{b}(z)$ is determined by the procedure outlined in Chapter 4 and the integral equation solved for $\alpha(z)$.

The solution of the integral equation will be dealt with in a later chapter. Here we will continue to establish the criterion for the applicability of the Born approximation.

\subsubsection{Region of validity of the Born approximation}

In studying the applicability of the Born approximation we start with the Schrodinger type equation (5.3). Putting $k(z)=k_{b}(z)+i \alpha(z)$ we obtain,

$$
\frac{d^{2} v(z)}{d z^{2}}+\left(k_{b}^{2}(z)+\mu_{b}(z)-k_{x}^{2}\right) v(z)=-2 i k_{b} \alpha(z) v(z)
$$

Let $G\left(z, z_{0}\right)$ be the outgoing Green's function satisfying the equation,

$$
\frac{d^{2} G}{d z^{2}}+\left(k_{b}^{2}(z)+\mu(z)-k_{x}^{2}\right) G=-\delta\left(z-z_{0}\right) \quad 0 \leq z, z_{0} \leq h
$$


Then we can write,

$$
v\left(k_{x}, z\right)=v_{b}\left(k_{x}, z\right)+\int_{0}^{h} 2 i k_{b}\left(z_{0}\right) \alpha\left(z_{0}\right) v\left(k_{x}, z_{0}\right) G\left(k_{x}, z, z_{0}\right) d z_{0}
$$

The total solution can therefore be viewed as the sum of the solution to the homogeneous equation $v_{b}(z)$ and a scattering part $v_{s}(z)$.

$$
v(z)=v_{b}(z)+v_{s}(z)
$$

Using the above, we obtain

$$
\begin{aligned}
v_{s}\left(k_{x}, z\right)= & \int_{0}^{h} 2 i k_{b}\left(z_{0}\right) \alpha\left(z_{0}\right) v\left(k_{x}, z_{0}\right) G\left(k_{x}, z, z_{0}\right) d z_{0} \\
v_{s}\left(k_{x}, z\right)= & \int_{0}^{h} 2 i k_{b}\left(z_{0}\right) \alpha\left(z_{0}\right) v_{b}\left(k_{x}, z_{0}\right) G\left(k_{x}, z, z_{0}\right) d z_{0}+ \\
& \int_{0}^{h} 2 i k_{b}\left(z_{0}\right) \alpha\left(z_{0}\right) v_{s}\left(k_{x}, z_{0}\right) G\left(k_{x}, z, z_{0}\right) d z_{0}
\end{aligned}
$$

In making the Born approximation we write $v(z)=v_{b}(z)$. This implies the following.

$$
\int_{0}^{h} m\left(z_{0}\right) v_{s}\left(k_{x}, z_{0}\right) G\left(k_{x}, z, z_{0}\right) d z_{0} \quad \ll v_{s}\left(k_{x}, z\right)
$$

where,

$$
m(z)=2 i k\left({ }_{b}(z) \alpha(z)\right.
$$

Define the norm of $f(z)$ as below. 


$$
\|f\|=\left\{\int_{0}^{h} f^{2}(z) d z\right\}^{1 / 2}
$$

Let $I$ represent the integral on the left hand side of the equation (5.21). Then,

$$
\|I\|=\left\{\int_{0}^{h}\left[\int_{0}^{h} m\left(z_{0}\right) v_{s}\left(k_{x}, z_{0}\right) G\left(k_{x}, z, z_{0}\right) d z_{0}\right]^{2} d z\right\}^{1 / 2}
$$

But, by Schwartz inequality,

$$
\begin{gathered}
\left|\int_{0}^{h} m\left(z_{0}\right) v_{s}\left(k_{x}, z_{0}\right) G\left(k_{x}, z, z_{0}\right) d z_{0}\right| \leq\left\{\int_{0}^{h} m\left(z_{0}\right) v_{s}\left(k_{x}, z_{0}\right) d z_{0}\right\} \\
\int_{0}^{h} G\left(k_{x}, z, z_{0}\right) d z_{0} \\
\left|\int_{0}^{h} m\left(z_{0}\right) v_{s}\left(k_{x}, z_{0}\right) G\left(k_{x}, z, z_{0}\right) d z_{0}\right| \leq\|m\|\left\|v_{s}\right\| \int_{0}^{h} G\left(k_{x}, z, z_{0}\right) d z_{0}
\end{gathered}
$$

The norm of $I$ therefore satisfies the inequality given below.

$$
\left.\|I\| \leq\|m\|\left\|v_{s}\right\|\left[\int_{0}^{h}\left\{\int_{0}^{h} G\left(k_{x}, z, z_{0}\right) d z_{0}\right)\right\}^{2} d z\right]^{1 / 2}
$$

Let $G\left(z, z_{0}\right)$ have a maximum value equal to $\left|G\left(z, z_{0}\right)\right|_{\max }$. Then,

$$
\|I\| \leq\|m\|\left\|v_{s}\right\| h\left|G\left(k_{x}, z, z_{0}\right)\right|_{\max }
$$

Using this in equation (5.27) we obtain,

$$
\|m\|\left\|v_{s}\right\| h\left|G\left(k_{x}, z, z_{0}\right)\right|_{m a x} \ll\left\|v_{s}\right\|
$$


or,

$$
\|m\| h\left|G\left(k_{x}, z, z_{0}\right)\right|_{\max } \ll 1
$$

The condition to be satisfied for Born approximation to be valid is then given by

$$
\left\|k_{b}\right\|\|\alpha\| h\left|G\left(k_{x}, z, z_{0}\right)\right|_{\max } \ll 1
$$

The applicability of the Born approximation depends on the magnitude of the perturbation, the depth of the sediment, and the magnitude of the Green's function. We will use this result in a later chapter to determine the most suitable angular aperture for the inversion scheme.

\subsection{Inverse problem formulation using the Rytov approximation}

We will in this section derive the integral equation for obtaining the unknown attenuation coefficient profiles using the Rytov approximation. We again start with the one-dimensional wave equation. For the sake of simplicity we have assumed that the density in the sediment layer is known and is a constant.

$$
\frac{d^{2} P(z)}{d z^{2}}+\left(k^{2}(z)-k_{x}^{2}\right) P(z)=0
$$

Let $P(z)=\exp (\psi(z))$. Substituting this in the above equation and using the relation $k(z)=k_{b}(z)+i \alpha(z)$, we obtain

$$
\frac{d^{2} \psi}{d z^{2}}+\left\{\frac{d \psi}{d z}\right\}^{2}+\left(k_{b}^{2}(z)-k_{x}^{2}\right)=-2 i k_{b}(z) \alpha(z)
$$

Let $P_{b}(z)$ be the solution to the wave equation for the background model. 


$$
\frac{d^{2} P_{b}}{d z^{2}}+\left(k_{b}^{2}(z)-k_{x}^{2}\right) P_{b}=0
$$

We now write $P_{b}(z)=\exp \left(\psi_{b}(z)\right)$. Substituting this in the wave equation yields the following.

$$
\frac{d^{2} \psi_{b}}{d z^{2}}+\left\{\frac{d \psi_{b}}{d z}\right\}^{2}+\left(k_{b}^{2}(z)-k_{x}^{2}\right)=0
$$

Let $\psi(z)=\psi_{b}(z)+\psi_{s}(z)$ : Substitute this in equation (5.33) and then subtract equation (5.35) from the result. We then have the following equation.

$$
\frac{d^{2} \psi_{s}}{d z^{2}}+2 \frac{d \psi_{s}}{d z} \frac{\psi_{b}}{d z}=-\left[\left(\frac{d \psi_{s}}{d z}\right)^{2}+2 i k_{b}(z) \alpha(z)\right]
$$

The left hand side of the above equation is, then, put into a more convenient form for manipulation as shown below.

$$
\frac{d^{2}\left(P_{b} \psi_{s}\right)}{d z^{2}}=\psi_{s} \frac{d^{2} P_{b}}{d z^{2}}+2 \frac{d \psi_{s} d P_{b}}{d z d z}+P_{b} \frac{d^{2} \psi_{s}}{d z^{2}}
$$

Using equation (5.34) and the relation $P_{b}(z)=P_{b}(z)\left(d \psi_{b}(z) / d z\right)$ the equation $(5.37)$ is written as below.

$$
\frac{d^{2}\left(P_{b} \psi_{s}\right)}{d z^{2}}+\left(k_{b}^{2}(z)-k_{x}^{2}\right) P_{b} \psi_{s}=P_{b}\left[\frac{d^{2} \psi_{s}}{d z^{2}}+2 \frac{d \psi_{b}}{d z} \frac{d \psi_{s}}{d z}\right]
$$

Now substitute equation (5.36) into the above to yield

$$
\frac{d^{2} P_{b} \psi_{s}}{d z^{2}}+P_{b} \psi_{s}\left(k_{b}{ }^{2}-k_{x}{ }^{2}\right)=P_{b}(z)\left[\left\{\frac{d \psi_{s}(z)}{d z}\right\}^{2}+2 i k_{b}(z) \alpha(z)\right]
$$

Multiply equation $(5.39)$ by $P_{b}(z)$ and equation $(5.34)$ by $P_{b}(z) \psi_{s}(z)$ and subtract one 
$-104-$

from the other. We then integrate over the depth $h$ and obtain the following equation.

$$
\begin{aligned}
\left.\left\{P_{b} \frac{d\left(P_{b} \psi_{s}\right)}{d z}-P_{b} \psi_{s} \frac{d P_{b}}{d z}\right\}\right|_{0}{ }^{h} & =\left.P_{b}^{2} \frac{d \psi_{s}}{d z}\right|_{0}{ }^{h} \\
& =-\int_{0}^{h}\left[\left(\frac{d \psi_{s}}{d z}\right)^{2}+2 i k_{b}(z) \alpha(z)\right] P_{b}^{2}(z) d z
\end{aligned}
$$

The left hand side of the equation is evaluated by relating it to the boundary conditions given in equations (5.9),(5.10) and (5.11). The relation between the $P$ s and $\psi$ s given below are also used for this purpose.

$$
\begin{aligned}
& \psi(z)=\ln P(z), \psi_{b}=\ln P_{b}(z), \psi_{s}=\ln \frac{P(z)}{P_{b}(z)} \\
& \frac{d \psi_{s}(z)}{d z}=\frac{1}{P(z)} \frac{d P(z)}{d z}-\frac{1}{P_{b}(z)} \frac{d P_{b}}{d z}
\end{aligned}
$$

Using the above we find that the left hand side of the equation vanishes at the upper limit, $z=h$. At the lower limit, $z=0$, it is given by the following expression.

$$
P_{b}^{2}(z) \frac{d \psi_{s}(0)}{d z}=2 i k_{z 0} \frac{R_{b}-R}{(1+R)\left(1+R_{b}\right)} \frac{\rho_{b}}{\rho_{0}}\left(1+R_{b}\right)^{2}
$$

The integral equation is then

$$
2 i k_{z 0} \frac{\rho_{b}}{\rho_{0}} \frac{1+R_{b}}{(1+R)}\left(R_{b}-R\right)=\int_{0}^{h}\left[\left(\frac{d \psi_{s}}{d z}\right)^{2}+2 i k_{b}(z) \alpha(z)\right] P_{b}^{2}(z) d z
$$

So far in our derivation no approximations have been made. We now make the approximation that $\left(d \psi_{s} / d z\right)^{2}$ is small compared to $2 k_{b}(z) \alpha(z)$. Neglecting this term the integral equation reduces to, 


$$
\left.\frac{k_{z 0}}{\rho_{0}} \frac{1+R_{b}}{1+R}\left(R_{b}-R\right)=\int_{0}^{h} \frac{1}{\rho_{b}} k_{b}(z) \alpha(z)\right] P_{b}^{2}(z) d z
$$

This equation is similar to the equation (5.14) obtained with the Born approximation except for a multiplicative factor in the left hand side.

\subsection{Summary}

In this chapter we have obtained the linear integral equation relating the unknown attenuation profile to the experimentally determined plane wave reflection coefficient. We have applied both the Born and the Rytov approximation to obtain two similar representation of the integral equation. Before we take up the solution of this integral equation, we will consider in the next chapter the general issues involved in solving this class of integral equations and include in it a review of the methods in the literature for obtaining solutions. 
$-106-$

\subsection{References}

1. E.L. Hamilton, "Geoacoustic modelling of the sea floor" J. Acoust. Soc. Am., 68(5), 1313-1340 (1980).

2. J.K. Cohen, and N. Bleistein, "An inverse method for determining small variations in propagation speed," SIAM Journal of Applied Mathematics, 32(4), 784-799 (1977).

3. J.B. Keller, "Accuracy and Validity of the Born and Rytov approximations," Journal of the Optical Society of America, 50, 1003-1004 (1969).

4. M.L. Oristaglio, "Accuracy of the Born and Rytov approximations for the laws of refraction and reflection at a plane interface," submitted to the Journal of the Optical Society of America, (1984). 


\section{Chapter 6 Linear Inverse Theory}

In Chapter 5, a non-linear integral equation was derived relating the attenuation coefficient profile to the reflection coefficients. The equation was linearised using the Born approximation and the linear equation obtained is given below.

$$
k_{z 0}\left\{R_{b}\left(k_{x}\right)-R\left(k_{x}\right\}=\int_{0}^{h k_{b}(z)} \frac{\rho_{b}(z)}{(z)} P_{b}^{2}\left(k_{x}, z\right) d z\right.
$$

A similar equation was obtained using the Rytov approximation. The unknown in the equation is $\alpha(\mathrm{z})$. The above equation can be written as

$$
d\left(k_{x}\right)=\int_{0}^{h} \alpha(z) G\left(k_{x}, z\right) d z
$$

where,

$$
\begin{aligned}
& d\left(k_{x}\right)=k_{z 0}\left\{R_{b}\left(k_{x}\right)-R\left(k_{x}\right)\right\} \\
& G\left(k_{x}, z\right)=\frac{k_{b}(z)}{\rho_{b}(z)} P_{b}^{2}\left(k_{x}, z\right)
\end{aligned}
$$

This is a Fredholm integral equation of the first kind. In this chapter we consider the issues of non-uniqueness and instability associated with the solution of this type of integral equations and the role the kernel plays in this. The equation given above assumes that $d\left(k_{x}\right)$ representing data are available continuously along a line. 
However, in practical situations $d\left(k_{x}\right)$ containing the data is known only at discrete points. Then the integral equation takes the following form wherethe n's represent the points at which the data is available.

$$
d_{n}=\int_{0}^{h} \alpha(z) G_{n}(z) d z
$$

In addition to being available only at discrete points, the data is contaminated by error as well. In this chapter we, therefore, review the methods in the literature for solving this class of equation with discrete and noisy data. For the purpose of the discussion that follows we will use a general form of the Fredholm integral equation given below.

$$
\int_{a}^{b} G(x, y) m(x) d x=d(y) \quad a \leq x \leq b, a \leq y \leq b
$$

\subsection{Fredholm integral equation of the first kind}

Consider the linear integral equation in equation (6.4). There are two aspects to be considered in the solution of this type of equations. These are (i) nonuniqueness of the solution, and (ii) the instability of the solution.

\subsubsection{Non-uniqueness}

The eigenvalue of the kernel is given by the equation

$$
\int_{a}^{b} G(x, y) \phi(x) d x=\lambda \phi(y)
$$

If the kernel has zero eigenvalues, then 


$$
\int_{a}^{b} G(x, y) \phi(x) d x=0
$$

If a non-trivial solution $\phi(x)$ exists for the above equation, then any multiple of $\phi(x)$ can be added to the solution of the equation and it will still be solution of the integral equation. There can thus be an infinite number of solutions for the integral equation.

\subsubsection{Instability}

By instability, we mean, that small changes in data can produce large changes in the solution. To demonstrate this property of the integral equation we use the proof due to Tikhonov[1].

Consider the integral equation (6.4). We shall measure changes in the function $d(y)$ by the norm defined below.

$$
l_{d}\left(d_{1}, d_{2}\right)=\left[\int_{a}^{b}\left\{d_{1}(y)-d_{2}(y)\right\}^{2} d y\right]^{1 / 2}
$$

The changes in $m(x)$ is measured by the following norm

$$
l_{m}\left(m_{1}, m_{2}\right)=\max .\left|m_{1}-m_{2}\right|
$$

Let us assume that the function $m_{1}(x)$ has been changed to $m_{2}(x)$ given by the following equation.

$$
m_{2}(x)=m_{1}(x)+N \sin (\omega x)
$$

Substituting in the integral equation, we obtain the change in $d(y)$. 


$$
d_{2}(y)=d_{1}(y)+N \int_{a}^{b} G(x, y) \sin \omega x d x
$$

The norm $l_{d}\left(d_{1}, d_{2}\right)$ is then,

$$
l_{d}\left(d_{1}, d_{2}\right)=\left\{\int_{a}^{b}\left[\int_{a}^{b} G(x, y) \sin \omega x d x\right]^{2} d y\right\}^{1 / 2}
$$

The norm $\mathrm{l}_{\mathrm{m}}\left(\mathrm{m}_{1}, \mathrm{~m}_{2}\right)$ is given by the follwing expression.

$$
l_{m}\left(m_{1}, m_{2}\right)=\max . N|\sin \omega x|=N
$$

For large values of $\omega$, the integral (6.11) approaches zero by virtue of ReimannLebesque lemma[2] as long as $\int_{a}^{b} G(x, y) d x$ exists. Therefore we can make $l_{d}\left(d_{1}, d_{2}\right)$ arbitrarily small while keeping $l_{m}\left(m_{1}, m_{2}\right)$ large.

To see how the kernel of the integral equation influences this property, we will consider a special type of integral equation for which we can obtain solution. Let us assume that the kernel of the integral equation is Hermitian. Then, by Hilbert-Schmit theorem[3] the kernel can be represented in terms of its eigenfunctions. The eigenfuctions are orthogonal and the eigenvalues are real.

$$
G(x, y)=\sum_{n=0}^{N} \lambda_{n} \phi_{n}(x) \phi_{n}^{*}(y)
$$

Substituting in equation, we obtain

$$
d(y)=\int_{a}^{b} m(x)\left\{\sum_{n=0}^{N} \lambda_{n} \phi_{n}(x) \phi_{n}^{*}(y)\right\} d x
$$

Let, 


$$
m(x)=\sum_{n=0}^{N} \alpha_{n} \phi_{n}(x)
$$

Substituting this in equation (6.14) and using the orthgonality property of the eigenfunctions we obtain

$$
d(y)=\sum_{n=0}^{N} \lambda_{n} \alpha_{n} \phi_{n}{ }^{*}(y)
$$

The coefficients $\alpha_{n}$ are obtained using the orthgonality property of the eigenfunctions.

$$
d_{n}=\lambda_{n} \alpha_{n} ; \alpha_{n}=\frac{d_{n}}{\lambda_{n}}
$$

where,

$$
d_{n}=\int_{a}^{b} d(y) \phi_{n}(y) d y
$$

Let $d(y)$ contain an error $e(y)$. Then,

$$
m(x)=\sum_{n=0}^{N} \frac{d_{n}+e_{n}}{\lambda_{n}} \phi_{n}{ }^{*}
$$

If the kernel has small eigenvalues then small error in data will result in large errors in solution. The kernel of the integral equation can be viewed as behaving like a smoothing operator which smooths out wide fluctuations in solution. Such smoothing operators are characterised by the presence of low eigenvalues. Indeed if the kernel is a delta function there will be no smoothing and a stable solution is obtained.

When data is available over an infinite range, unique solution is possible if the 
$-112-$

kernel has no zero eigenvalue. One example of this is the Fourier integral which yields an unique solution. However, in practical situations, data is available only at a limited number of points. This situation generally yields non-unique solutions. We have to, therfore, deal with the twin problem of instability and non-uniqueness in solution. Many methods have been proposed in the literature for solving equations of this type. All of them incorporate a priori information about the solution to overcome the problem of non-uniqueness. The solution is made stable by schemes which in effect make the eigenvalues large or reduce the effect of the small eigenvalues. We will review these methods, briefly, in the following paragraphs. We will also study how solutions are obtained when the data contains noise.

When data is available only at discrete points the integral equation takes the form in equation (6.3). In solving the integral equation two approaches are possible. The integral can be represented as a sum by adopting a suitable quadrature scheme and then the solution of the integral equation reduces to solving a set of linear equations. A reasonable quadrature scheme can be adopted if we have some prior knowledge of the solution from the physics of the problem. For example, if we know that the solution is smoothly varying we can assume without much error that the function is constant over small intervals and represent the solution by a set of parameters. On the other hand, if we have no knowledge about the solution, such discretisation can be erroneous since it may hide some structure that exists in the solution unless the discretisation is made infinitely small. An alternative is, then, to express the solution as linear sum of a set of basis functions and determine the coefficients of the linear combination. The class of methods which follows the first approach we will call as providing solution in parameter space. The latter we will call as solution in function space. 


\subsection{Solution in parameter space - Error free data}

By using a suitable quadrature scheme the integral (6.3) is represented by the sum given below.

$$
d_{i}=\sum_{j} w_{i j} G_{i j} m_{j}
$$

where $w_{i j}$ is the weighting associated with the quadrature scheme. For the entire set of observations we obtain the following matrix equation.

$$
\mathrm{d}=\mathbf{G m}
$$

where $d$ is a $n x 1$ vector containing the observations and $\mathbf{G}$ is an nxm matrix which operates on the vector $\mathbf{m}$ of dimension $\mathbf{m x} 1$ representing the solution.

\subsubsection{Generalised Inverse}

If $G$ is a nxn non-singular matrix, then, the solution to the equation is easily obtained.

$$
\mathbf{m}=\mathbf{G}^{-1} \mathbf{d}
$$

When the integral is written as a sum, the interval $\Delta x$ is made small so that the true structure of the solution is obtained. This results in an under-determined system. Though the analysis which follows is applicable for any general nxm matrix we will consider such a case i.e. where $\mathrm{n}$ is less than $\mathrm{m}$. It can be shown[4] that the square matrices $\mathbf{G G}^{T}$ and $\mathbf{G}^{T} \mathbf{G}$ define a coupled eigenvector eigenvalue problem such that

$$
\mathrm{GG}^{T} \mathbf{u}_{i}=\lambda_{i}^{2} \mathbf{u}_{i}
$$




$$
\mathrm{G}^{T} \mathrm{Gv}_{i}=\lambda_{i}^{2} \mathbf{v}_{i}
$$

and

$$
\begin{aligned}
& \mathbf{G v}_{i}=\lambda_{i} \mathbf{u}_{i} \\
& \mathbf{G}^{T_{\mathbf{u}_{i}}}=\lambda_{i} \mathbf{v}_{i}
\end{aligned}
$$

In addition there exists $\mathrm{m}-\mathrm{n}$ eigenvectors $\mathbf{v}_{\mathbf{i}}$ such that $\mathbf{G v}_{i}=0, i=n+1, \ldots \ldots, m$. Lanczos[4] has shown that the matrix $\mathbf{G}$ can be decomposed into product of three matrices. Using the decomposition theorem we obtain

$$
\mathbf{G}=\mathrm{U}_{N} \Lambda_{N} \mathbf{v}_{N}^{T}
$$

Here $U_{N}$ is an nxn matrix containing the eigenvectors of $G^{T} G, V_{N}$ is an $m x n$ matrix containing the eigenvectors of $\mathbf{G}^{\mathrm{T}} \mathbf{G}$ for the non-zero eigenvalues. $\Lambda$ is a diagonal matrix containing the non-zero eigenvalues. Since $\mathbf{G v}_{\mathrm{i}}=\mathbf{0}$ for $\mathrm{i}=\mathrm{n}+\mathbf{1}, \ldots$ , $\mathrm{m}$, any linear combination of these can be added to the solution and the data will still be satisfied. This is the cause of non-uniqueness in an under-determined system.

The operator defined below is called the generalised inverse operator.

$$
\mathrm{G}_{g}^{-1}=\mathrm{V}_{N} \Lambda^{-1} \mathrm{U}_{N}^{T}
$$

Using this operator we obtain

$$
\mathbf{m}_{g}=\mathbf{V}_{N^{\Lambda^{-1}}{ }_{N} \mathrm{U}_{N}{ }^{T} \mathbf{d}}
$$

or 


$$
\mathbf{m}_{g}=\sum_{i=1}^{n} \alpha_{i} \mathbf{v}_{i}
$$

The solution $\mathbf{m}_{g}$ obtained by the generalised inverse operator, therefore, uses only the eigenvectors corresponding to the non-zero eigenvalues. The true solution however will be a linear combination of all the eigenvectors. We can then represent the true solution by the following equation.

$$
\mathbf{m}=\sum_{i=1}^{n} \alpha_{i} \mathbf{v}_{i}+\sum_{j=n+1}^{m} \beta_{j} \mathbf{v}_{j}
$$

The norm of the total solution is then,

$$
|\mathbf{m}|^{2}=\left|\mathbf{m}_{g}\right|^{2}+\sum_{j=n+1}^{m} \beta_{j}^{2}
$$

From the above we see that the generalised inverse operator yields a solution with minimum norm.

Though the problem of non-uniqueness has been resolved instability still exists. Consider the situation when data contains error. Then the error in solution is given by the following expression.

$$
\Delta \mathbf{m}_{g}=\mathbf{G}_{g}^{-1} \Delta \mathbf{d}
$$

The covariance of the error in the solution can then be obtained. Here E[.] represents the averaging operator.

$$
E\left[\Delta \mathbf{m}_{g} \Delta \mathbf{m}_{g}^{T}\right]=\mathbf{G}_{g}^{-1} E\left[\Delta \mathbf{d} \Delta \mathbf{d}^{T}\right] \mathbf{G}_{g}^{-T}
$$

Assuming that the elements of $\Delta \mathbf{d}$ are statistically independent and have the same variance we obtain, 


$$
E\left[\Delta \mathbf{m}_{g} \Delta \mathbf{m}_{g}{ }^{T}\right]=\sigma_{d}{ }^{2} \mathrm{~V}_{N} \Lambda_{N}^{-2} \mathrm{~V}_{N}{ }^{T}
$$

We note that the error in solution is propotional to $1 / \lambda_{n}{ }^{2}$ and the variance becomes large when the eigenvalues are small. Wiggins[5] and Jackson[6] suggest that the effect of low eigenvalues can be eliminated if we set eigenvalues lower than a threshold level equal to zero. The eigenvectors associated with these eigenvalues are therefore eliminated and the solution obtained will therefore have poorer resolution. Eliminating eigenvectors corresponding to small eigenvalues is equivalent to obtaining a smooth solution.

\subsubsection{Regularisation method}

Phillips[7], Twomey[8], and others have proposed regularisation methods for obtaining an unique stable solution. In the generalised inverse method we obtained an unique stable solution by looking for a solution which has a specific property namely that of minimum norm. The instability was overcome by effectively smoothing the solution. The regularisation method proposed by Twomey[8] is based on the method first suggested by Phillips [7] and looks for a smooth solution. The measured data $d(y)$ is available at discrete points and therfore it is defined only at these points and to within measurement error as shown in figure 6-1. We can therefore say that $d(y)$ is arbitrary except that it passes within the error bars associated with each of the measurement points. Therefore, there can be an infinite set of $m(x)$ associated with the infinite set of $d(y)$. The ambiguity can only be overcome by imposing additional constraints on $m(x)$ which enables one to pick out of the large possible set of $m(x)$ one that satisfies the conditions imposed on the solution. One such constraint that can be imposed is the smoothness criterion. 

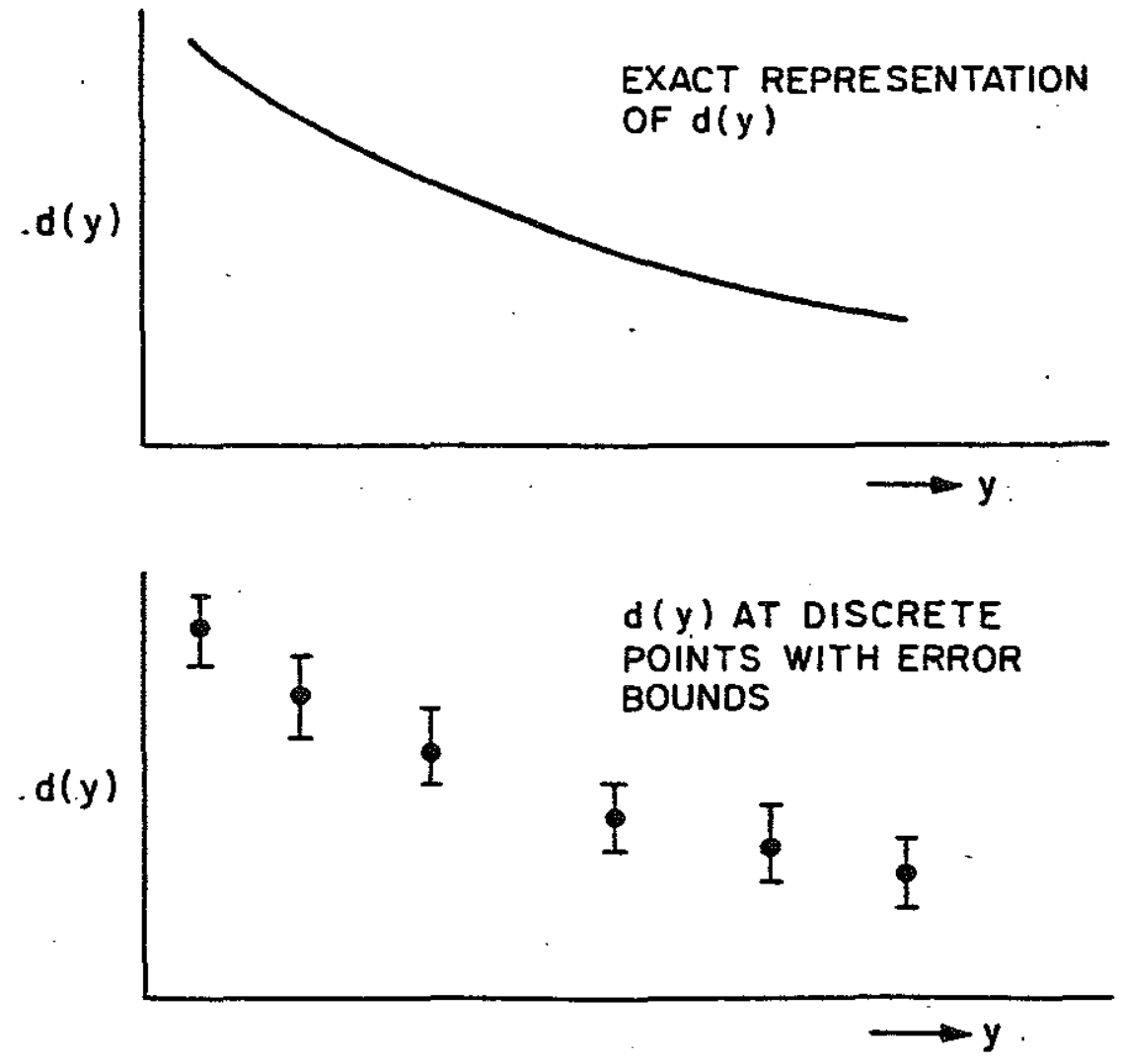

Figure 6-1: 
$-118-$

Though the data does not in any way suggest that the solution $m(x)$ must be smooth function, we apriori make this assumption. There could be other constraints. But one selects the constraint from the physical nature of the problem and ones guess of what the solution should be.

Let $S(m(x))$ be a measure of smoothness of the solution i.e the smaller the value of $S$, the smoother $m(x)$ is. Restating the problem we now try to find $m(x)$ that minimises $S(m(x))$ subject to the data constraints being met. A least squares procedure can now be adopted which minimises $|\mathbf{d}-\mathbf{G m}|^{2}+\lambda S(m(x))$.

One measure of smoothness that is proposed by Twomey[9] is the quadratic measure given below.

$$
S(m(x))=\int_{a}^{b}\left(m^{\prime \prime}(x)\right)^{2} d x
$$

We can generalise this by considering a constraint operator $\mathbf{D}$ acting on $\mathbf{m}$. We, then, seek to minimise the quadratic measure defined below.

$$
\mathbf{m}^{T} \mathbf{D}^{T} \mathbf{D m}=\mathbf{m}^{T} \mathbf{H m}
$$

where $\mathbf{H}=\mathbf{D}^{T} \mathbf{D}$. To obtain a solution for $\mathbf{m}$ we now minimise the expression;

$$
\mathbf{m}^{T} \mathbf{H m}+\lambda|\mathbf{d}-\mathbf{G m}|^{2}
$$

Differentiating with respect to each element of $\mathbf{m}$, we obtain the solution;

$$
\left(\mathbf{G}^{T} \mathbf{G}+\lambda \mathbf{H}\right) \mathbf{m}=\mathbf{G}^{T_{\mathbf{d}}}
$$

We note that the acceptable solution has now been defined as one which satisfies the smoothness constraint. By the addition of the matrix $H_{\text {to }} \mathbf{G}^{\mathrm{T}} \mathbf{G}$ we 
have in effect increased the magnitude of the eigenvalues. This results in a stable and smooth solution.

To see how the addition of a matrix like $\mathbf{H}$ affects the eigenvalue, we investigate the case when $\mathbf{H}=\mathbf{I}$ the identity matrix. Consider the eigenvalue problem for $\mathbf{G}^{\mathrm{T}} \mathbf{G}$. Then,

$$
\mathrm{G}^{T} \mathrm{Gv}=\lambda \mathbf{v}
$$

where $\lambda$ is the eigenvalue. Adding (eI)v to both sides, we obtain

$$
\left(\mathbf{G}^{T} \mathbf{G}+e \mathbf{I}\right) \mathbf{v}=(\lambda+e \mathbf{I}) \mathbf{v}
$$

The eigenvalue problem for $\left(\mathbf{G}^{\mathrm{T}} \mathbf{G}+\mathrm{eI}\right)$ therefore gives the same eigenvectors but the eigenvalue has been increased by eI. The solution obtained by the regularisation method when $\mathbf{H}=\mathbf{I}$ is given below.

$$
\mathbf{m}=\left(\mathrm{G}^{T} \mathrm{G}+e \mathrm{I}\right)^{-1} \mathrm{G}^{T} \mathbf{d}
$$

By eigenvector analysis it can be shown that the inverse operator shown above has the following form.

$$
\left(\mathbf{G}^{T} \mathbf{G}+e \mathbf{I}\right)^{-1}=\mathbf{V}_{n}\left\{\frac{\Lambda_{n}}{\Lambda_{n}{ }^{2}+e}\right\} \mathbf{U}_{n}^{T}
$$

We therefore see that when the eigenvalues are small, i.e $\lambda_{n} \ll e$ the diagonal terms of the matrix goes as $\left(\lambda_{n} / e\right)$, thereby eliminating the effect of small eigenvalues. On the otherhand when $\lambda_{n} \gg e$ then the effect of $\mathrm{e}$ is ignored. 


\subsection{Solution in parameter space-Data with errors}

\subsubsection{Generalised Inverse}

So far we considered error free data. However, in any measurement there will be error. We can weight each observation depending on the variance of the measurement. If $\sigma_{i}^{2}$ is the variance of the ith measurement, we weight the ith observation by $1 / \sigma_{i}$. Then each observation is related to the solution by the following equation.

$$
\frac{d_{i}}{\sigma_{i}}=\frac{1}{\sigma_{i}} \sum^{m} g_{i j} m_{j}
$$

We see that the data with the least variance will have the highest weightage. Let $\mathbf{S}$ be the covariance matrix for the data. If the errors at each observation are statistically independent, the covariance matrix is a diagonal matrix with the diagonal terms equal to $1 / \sigma_{i}{ }^{2}$. Then, for a set of observation we obtain the following matrix relationship.

$$
\mathrm{S}^{-1 / 2} \mathbf{d}=\mathbf{S}^{-1 / 2} \mathrm{Gm}
$$

We now define a new matrix $\mathbf{G}_{e}=\mathbf{S}^{-1 / 2} \mathbf{G}$ and a vector $\mathbf{d}_{e}=\mathbf{S}^{-1 / 2} \mathbf{d}$. We, then, obtain the solution using the generalised inverse method. If we have some information about the variance of the elements of the solution vector these can be incorporated in the solution by multiplying each column of the matrix $G$ by a weight $1 / \sigma_{\mathrm{mj}}$. The equation to be solved then takes the following form.

$$
\mathbf{d}^{\prime}=\mathbf{G}^{\prime} \mathbf{m}^{\prime}
$$

where, 


$$
\begin{aligned}
& \mathbf{d}^{\prime}=\mathbf{S}^{-1 / 2} \mathbf{d} \\
& \mathbf{G}^{\prime}=\mathbf{S}^{-1 / 2} \mathbf{G} \mathbf{T}^{1 / 2} \\
& \mathbf{m}^{\prime}=\mathbf{T}^{-1 / 2} \mathbf{m}
\end{aligned}
$$

$\mathbf{T}$ is a diagonal matrix containing $\sigma_{\mathrm{m}}{ }^{2}$ along its diagonal. Using these relations a generalised inverse solution is obtained as before. Jackson[6] has shown that even if the covariance matrices are not diagonal a solution can be obtained which incorporates the information on the covariance of the data and the solution.

\subsubsection{Regularisation Method}

As in the previous method, we weight each equation and obtain a transformed matrix equation. Using this transformed equation and the procedure outlined earlier for obtaining a regularised solution we obtain[9], the following solution.

$$
\mathbf{m}=\left(\mathbf{G}^{T} \mathbf{S}^{-1} \mathbf{G}+\lambda \mathbf{H}\right)^{-1} \mathbf{G}^{T} \mathbf{S}^{-1} \mathbf{d}
$$

\subsubsection{Maximum likelihood estimate}

The data measurements are considered as a set of random variables. If we make tha assumption that the random variables are distributed in a Gaussian manner and $\mathbf{S}$ is the covariance matrix for these variables, then the probability density function for the data is given by the following equation.

$$
p(\mathbf{d})=\frac{\left|\mathbf{S}^{-1}\right|^{1 / 2}}{(2 \pi)^{N / 2}} \exp \left\{-1 / 2(\mathbf{d}-\mathbf{G m})^{T} \mathbf{S}^{-1}(\mathbf{d}-\mathbf{G m})\right\}
$$


For maximising the probability $p(d)$, we need to minimise $(d-G m)^{T} S^{-1}(d-G m)$. By a similar argument. we can say that the minimisation of $\mathrm{m}^{\mathrm{T}} \mathrm{m}$ assuciated with the generalised inverse can be replaced by $\mathbf{m T}^{-1} \mathbf{m}$. The solution can then be obtained by the generalised inverse in transformed co-ordinates as discussed in earlier paragraphs.

\subsubsection{Minimum varaince estimate}

In this approach also the data and the solution vectors are treated as a random vectors. In the minimun variance estimate we seek a linear operator which gives an unbiased minimum variance estimate for the sulution. By GaussMarkov[10] theorem the minimum variance unbiased estimate is giren by the following equation.

$$
\mathbf{m}=\mathbf{C}_{m d} \mathbf{C}_{d d}{ }^{-1} \mathbf{d}
$$

where,

$$
\begin{aligned}
& \mathbf{C}_{m d}=E\left[\mathbf{m d}^{T}\right] \\
& \mathbf{C}_{d d}=E\left[\mathbf{d d}^{T}\right]
\end{aligned}
$$

Consider now the matrix equation given below.

$$
\mathbf{d}=\mathbf{G m}+\mathbf{e}
$$

Herse e represents noise.

Using equation (6.49) and assuming that the noise and the solution vector are statistically independent we obtain[10] 


$$
\mathbf{m}=\left(\mathbf{G} \mathbf{C}_{e e}^{-1}+\mathbf{C}_{m m}^{-1}\right) \mathbf{G}^{T} \mathbf{C}_{e e^{-1} \mathbf{d}}
$$

where,

$$
\begin{aligned}
& \mathbf{C}_{e e}=E\left[\mathrm{ee}^{T}\right] \\
& \mathbf{C}_{m m}=E\left[\mathbf{m m}^{T}\right]
\end{aligned}
$$

The stochastic inverse method proposed by Franklin[11] also yields the same result.

We note that approximate solutions are obtained in these methods by incorporating apriori information about the statistics of the solution. Assumption on the covariance of the solution is equivalent to assumptions on its smoothness made in regularisation method. Further addition of a positive definite matrix like $\mathrm{C}_{\mathrm{ee}}^{-1}$ overcomes the problem of instability.

\subsection{Solution in function space- Error free data}

\subsubsection{Spectral expansion method}

Parker[12], arguing that the inverse problem differs from parameter estimation problem in that the unknown is a function with an infinite number of parameters, has provided a solution in the function space. This method is based on the fact that since $d_{i}$ is the projection of $m(x)$ on $G_{i}(x)$ we can construct a set of

orthogonal functions to represent $G(x)$ and express $m(x)$ as linear combination of these orthogonal functions. To construct the orthogonal functions, a covariance matrix $\Gamma$ is formed. 


$$
\Gamma_{i j}=\int_{a}^{b} G_{i}(x) G_{j}(x) d x
$$

$\Gamma$ is a symmetric matrix and if the equations are linearly independent the eigenvalues are real and positive. $\Gamma$ is now expressed as a product of three matrices using the decomposition theorem[4].

$$
\Gamma=\mathbf{V} \Lambda \mathbf{V}^{T}
$$

Here the matrix $\mathbf{V}$ contains the eigenvectors and $\Lambda$ is a diagonal matrix containing the eigenvalues. The orthogonal functions are then formed by using the following equation.

$$
\phi_{i}(x)=\lambda_{i}^{-1 / 2} \sum_{j} v_{i j} G_{j}(x)
$$

Expressing the unknown function as a linear combination of the orthogonal function we write;

$$
m(x)=\sum_{i=1}^{N} a_{i} \phi_{i}(x)+\phi^{*}(x)
$$

where $\phi^{*}(\mathrm{x})$ are orthogonal to $\phi(\mathrm{x})$. Multiplying both sides by $\phi(\mathrm{x})$ and integrating over the domain a to $b$ we obtain

$$
a_{i}=\int_{a}^{b} m(x) \phi_{i}(x) d x
$$

or,

$$
a_{i}=\lambda_{i}^{-1 / 2} \sum_{j} v_{i j} d_{j}
$$


Having obtained $a$ 's, $m(x)$ is then evaluated using equation

$$
m(x)=\sum_{i=1}^{N} a_{i} \phi_{i}
$$

Using equation (6.54) we now compute the norm of $m(x)$.

$$
\|m(x)\|^{2}=\int_{a}^{b}\left[\sum_{i=1}^{N} a_{i} \phi_{i}(x)+\phi^{*}(x)\right]^{2} d x
$$

or,

$$
\|m(x)\|^{2}=\mathbf{a a}^{T}+\left\|\phi^{*}\right\|^{2}
$$

By constructing the solution using only $\phi_{i}$ and neglecting the functions in the space orthogonal to it we obtain the minimum norm solution as in the case of generalised inverse. As the $a_{i}$ 's are propotional to $\lambda_{i}^{-1 / 2}$, small eigenvalues will magnify noise. As done before, we set the eigenvalue as zero if its value is less than a threshold level and their eigenfunctions deleted from the solution. A method for determining how many eigenfuction are to be included in the solution is given by Parker[12].

\subsubsection{Method of Backus and Gilbert}

The importance that the kernel of the integral equation in obtaining an unique stable soluion has been discussed earlier. In the method proposed by Backus and Gilbert $[13,14]$, the kernel of the equation is made close to a delta function. Consider the equation given below.

$$
d_{i}=\int_{a}^{b} G_{i}(x) m(x) d x \quad i=1, \cdots, N
$$


If by suitable linear combination of the kernel we make it close to a delta function at some point $x_{0}$ then the value of the solution at this value of $x$ is easily determined. Let $a_{\mathrm{i}}$ represent coefficient of the linear combination. Summing over all $a_{\mathrm{i}}$ s we obtain

$$
\sum_{i=1}^{N} a_{i}\left(x_{0}\right) d_{i}=\int_{a}^{b}\left\{\sum_{n=1}^{N} a_{i}\left(x_{0}\right) G_{i}(x)\right\} m(x) d x
$$

or

$$
\sum_{i=1}^{N} a_{i}\left(x_{0}\right) d_{i}=\int_{a}^{b} A\left(x, x_{0}\right) m(x) d x
$$

If $A\left(x, x_{0}\right)$ is a delta function $\delta\left(x-x_{0}\right)$ then $m\left(x_{0}\right)$ is equal to the left hand side of the equation above. However, it is not possible to construct such delta function. We, therefore, choose the coefficients $a_{i}$ in such a way that the function $A\left(x, x_{0}\right)$ approaches a delta function in a least squared sense. Various measures of 'deltaness' (spread function) have been proposed by the authors[14]. For the purpose of our discussion we will define the spread function as given below.

$$
S=\int_{a}^{b}\left\{A\left(x, x_{0}\right)-\delta\left(x-x_{0}\right)\right\}^{2} d x
$$

We make the additional assumption that the function $A\left(x, x_{0}\right)$ is unimodular i.e. $\int_{a}^{b} A\left(x, x_{0} d x=1\right.$. By using a Lagrange multiplier, we then determine the coefficients which minimises the spread subject to the unimodular constraint. Having determined the coefficients, these are substituted in equation $\sum_{i=1}^{N} a_{i} d_{i}$ and the value of the solution at $x_{0}$ determined. 
$-127-$

\subsection{Solution in function space- Data with errors}

The spectral method discussed above can be extended to take into account errors in data by weighting each equation. Another approach which incorporates the stochastic information of the data and the solution has been given by Tarantola and Nercessian [15] and Tarantola and Valette[16]. Tarantola and Nercessian[15] show that their general result reduces to the Backus and Gilbert result if there is no error in data and if we make the assumption that no information exists about solution.

\subsection{Non-linear problem}

So far we have dealt with the linear problem. The approach in solving the nonlinear problem is to linearise it by expanding the kernel around a base value which is assumed known. We start with the non-linear equation given below.

$$
d_{i}=G(m(x), x)
$$

Now we expand $G_{i}(x, m(x))$ around $m_{0}(x)$ which is close to $m(x)$ such that $m(x)=m_{0}(x)+\delta m(x)$. If we can write,

$$
\begin{aligned}
d_{i}= & \int_{a}^{b} G_{i}\left(m_{0}(x), x\right) d x+ \\
& \int_{a}^{b} D_{i}\left(m_{0}(x), x\right) \delta m(x) d x+O\|\delta m(x)\|^{2}
\end{aligned}
$$

then $G_{i}(x, m(x))$ is said to be Frechet differentiable at $m_{0}(x)$. Omitting the higher order terms we can write 


$$
d_{i}-\left(d_{0}\right)_{i}=\Delta d_{i}=\int_{a}^{b} D_{i}(x) \delta m(x) d x
$$

and since $m_{0}(x)$ is known the equation is now in linear form and we can apply the techniques described in this chapter.

The solution of non-linear equation starts with a guess of the solution and the kernel is expanded about this and a correction is obtained. Using this we obtain a new estimate of the solution which can now be used as the background. We note that such linearisation is permissible only if the kernel is Frechet differentiable.

\subsection{Summary}

In this chapter we have reviewed the various approaches available in literature for solving Fredholm integral equation of the first kind when the dats are available only at discrete points and is contaminated by error. In the next chapter we take up the solution of the integral equation (6.1) and obtain the attenuation coefficient profile for the ocean bottom. 


\subsection{References}

1. A.N. Tikhonov and V.Y. Arsenin,Solution of ill-posed problems, (Wiley, Newyork, 1977).

2. C.M. Bender and S.A. Orszag,Advanced mathematical methods for scientists and engineers, (McGraw-Hill, New York, 1978).

3. P.M. Morse and H. Feshbach, Methods of Theoretical Physics, (McGraw-Hill, New York, 1953).

4. C. Lanczos, Linear differential operators, (Van Norstrand, New York, 1961).

5. R.A. Wiggins, "The general linear inverse problem: implication of surface waves and free oscillations for the earth structure," Rev. Geophysical and Space Physics, 10, 251-285 (1972).

6. D.D. Jackson, "Interpretation of inaccurate, insufficient and inconsistant data," Geophys. J. R. Astr. Soc., 23, 125-138 (1971).

7. D.L. Phillips, "A technique for the numerical solution of Fredholm integral equation of the first kind," J. Assoc. Compt. Mach., 9, 84-97 (1962).

8. S. Twomey, "On the numerical solution of Fredholm integral equation of the first kind by inversion of the linear system produced by quadrature," J. Assoc. Compt. Mach., 10, 97-101 (1963).

9. S. Twomey, Introduction in the mathematics of inversion in remote sensing and indirect measurement, (Elsevier, New York, 1977).

10. P. Weibelt, An introduction to optimal estimation,(Addison-Wiley, Reading, 1967)

11. J.N. Franklin, "Well-posed stochastic extension of ill-posed problem," J. Math. Anal. Appl., 31, 682-716 (1970).

12. R.L. Parker," Understanding inverse theory," Ann. Rev. Earth Planet. Sci., 5, 35-64, (1977).

13. G.E. Backus and J.F. Gilbert,"Numerical application of a formalism 
for geophysical inverse problems," Geophys. J. R. Astr. Soc., 13, 247-276 (1967).

14. G.E. Backus and J.F. Gilbert, "The resolving power of gros earth data," Geophys. J. R. Astr. Soc., 16, 169-205 (1968).

15. A. Tarantola and A. Nercessian, "Three dimensional inversion without blocks," Geophys. J. R. Astr. Soc., 76, 299-306 (1984).

16. A. Tarantola and B. Valette, "Inverse problems $=$ Quest for information," Journal of Geophysics, 50, 159-170, (1982). 


\section{Chapter 7 \\ Inversion for Acoustic Parameters}

In this chapter we demonstrate the inversion procedure for obtaining the attenuation profile for the ocean bottom. We show that by suitably modifying the scheme corrections to the sound speed profile and density profile for the bottom can be obtained in addition to the attenuation profile.

First we establish that the kernel of the non-linear integral equation is Frechet differentiable and that the kernel obtained by the Born approximation is a Frechet kernel. The iteration procedure for solving the non-linear problem is then obtained.

We select the most suitable angular aperture for input information based on the region of applicability of the Born approximation. We study the effect that frequency has on the ability to reconstruct so that this information can be used in the design of the experiment. Similarly we study the effect of the magnitude and the extent of the perturbation on the performance of the inversion scheme. We follow this by demonstrating the reconstruction of different types of profiles using synthetically generated data. A comparison between results obtained with Born and Rytov approximation is then made.

Reconstruction of the attenuation coefficient profile is done by using the regularisation method mentioned in Chapter 6. We have not attempted to carry out the inversion by all the methods described in Chapter 6 and choose one that gives the best results. On the other hand, we have been guided by the fact that the function we are trying to reconstruct is a smooth function. The regularisation 
$-132-$

method which uses the smoothness of the model as one of the constraints has a strong appeal in this context and we have therefore chosen this approach. The results obtained using this method are promising.

The means of measuring the performance of the inversion is developed in the context of the resolving power theory of Backus and Gilbert[1]. Using this we study the resolution of the inversion performed.

In studying the question of errors, we deal with two aspects of it. First we consider the situation where the sound speed profile and the density profile are not known exactly. Instead of treating this difference between the exact profile and the assumed one as an error, we treat it as an unknown and include it as one of the functions to be determined. We demonstrate this by simultaneousiy inverting for the attenuation profile and corrections to sound speed profile. We also study the effect of adding noise to the data. Examples of reconstruction using noisy data are presented.

\subsection{Linearisation asd iteration procedure}

In Chapter 5 we obtained a non-linear integral equation and used the Born approximation to linearise it. We will be using this linear integral equation for performing the inversion. We now show that within the region of validity of the Born approximation this approximation is consistant with the requirement of Frechet differentiability of the non-linear kernel. To establish that the kernel of the non-linear integral equation is Frechet differentiable we follow the analysis of Parker[2]. The integral equation relating the unknown function to the reflection coefficient is given by the following equation. 


$$
k_{z 0}\left\{R_{b}\left(k_{x}\right)-R\left(k_{x}\right)\right\}=\int_{0}^{h} \frac{k_{b}(z)}{\rho_{b}(z)} \alpha(z) P\left(k_{x}, z\right) P_{b}\left(k_{x}, z\right) d z
$$

In obtaining the above equation we have assumed that the value of the attenuation coefficient for the background model is zero. We now write $P\left(k_{x}, z\right)=P_{b}\left(k_{x}, z\right)+P_{s}\left(k_{x}, z\right)$ where $P_{b}\left(k_{x}, z\right)$ is the solution when $\alpha(z)=0$. Substituting in the above equation we obtain,

$$
k_{z 0}\left(R_{b}\left(k_{x}\right)-R\left(k_{x}\right)\right)=\int_{0}^{h} \gamma(z) P_{b}\left(k_{x}, z\right)^{2} d z+S\left(k_{x}\right)
$$

where

$$
\begin{aligned}
& \gamma(z)=\frac{k_{b}(z)}{\rho_{b}(z)} \alpha(z) \\
& S\left(k_{x}\right)=\int_{0}^{h} \gamma(z) P_{b}\left(k_{x}, z\right) P_{s}\left(k_{x}, z\right) d z
\end{aligned}
$$

In making the Born approximation we assume that $S\left(k_{x}\right)$ is small in comparison to the first term and neglect it. Comparing equation (7.2) with equation (6.64) in Chapter 6, we note that for Frechet differentiability, $S\left(k_{x}\right)$ must be of the order of $\|\gamma\|^{2}$.

Define the norm for $\gamma(z)$ as given below.

$$
\|\gamma\|=\left\{\int_{0}^{h}[\gamma(z)]^{2} d z\right\}^{1 / 2}
$$

Applying the Schwartz inequality to $S\left(k_{x}\right)$ we find that, 


$$
\|S\| \leq\left\|\gamma P_{\ell}\right\|\left\|P_{s}\right\|
$$

To obtain an etimate of $\left\|P_{s}\right\|$ we asssume that within the region of validity of Born approximation $P_{s}\left(k_{x}, z\right)$ can be written as follows.

$$
P_{s}\left(k_{x}, z\right)=\int_{0}^{h} \gamma\left(z^{\prime}\right) P_{b}\left(k_{x^{\prime}}, z^{\prime}\right) G\left(k_{x}, z, z^{\prime}\right) d z^{\prime}
$$

where $G\left(z, z^{\prime}\right)$ satisfies the equation;

$$
\frac{d^{2} G}{d z^{2}}+\left(k_{b}{ }^{2}(z)-k_{x}{ }^{2}\right) G=\delta\left(z-z^{\prime}\right)
$$

Applying Schwartz inequality we obtain,

$$
\left\|P_{s}\right\| \leq\|\gamma\|\left\|P_{b}\right\|\left\{\int_{0}^{h}\left(G\left(z, z^{\prime}\right)\right)^{2} d z\right\}^{1 / 2}
$$

Using this we find that,

$$
\|S\| \leq\|\gamma\|^{2}\left\|P_{b}\right\|^{2}\left\{\int_{0}^{h}\left(G\left(z, z^{\prime}\right)\right)^{2} d z\right\}^{1 / 2}
$$

The field $P_{b}\left(k_{x}, z\right)$ in the sediment layer is finite. We will show in a later section of this chapter that in the range of angles chosen for input data, the Green's function also remains finite. The equation (7.8) can, then, be written as given below.

$$
\begin{aligned}
& \|S\| \leq C\|\gamma\|^{2} \\
& \|S\| \leq O\|\gamma\|^{2}
\end{aligned}
$$

We have, therfore, established that the kernel is Frechet differentiable and that the 
$-135-$

kernel obtained by Born approximation is a Frechet kernel.

Linearising of the integral equation is based on the term $S\left(k_{x}\right)$ being negligible. If this is not so, then the solution when used in the non-linear integral equation will not satisfy data. We, therefore, need to use an iterative process. In the iterative scheme the solution obtained in the previous iteration is used to generate the new kernel. The integral equation then takes the form shown below.

$$
\begin{aligned}
& k_{z 0}\left\{R_{n}\left(k_{x}\right)-R\left(k_{x}\right)\right\}=\int_{0}^{h k_{b}(z)} \frac{\rho_{b}(z)}{\rho_{n}} \delta \alpha_{n}(z) P_{b n}{ }^{2}\left(k_{x}, z, \alpha_{n}(z)\right) d z \\
& \alpha_{n+1}(z)=\alpha(z)+\delta \alpha_{n}(z)
\end{aligned}
$$

A variant of this is the fixed point iteration. In this case we first write,

$$
\delta \alpha(z)=\alpha(z)-\alpha_{n}(z)
$$

Substituting this in equation (7.12) we obtain,

$$
\begin{aligned}
& k_{z 0}\left\{R_{n}\left(k_{x}\right)-R\left(k_{x}\right\}+\int_{0}^{h k_{b}(z)} \frac{\rho_{b}(z)}{n} \alpha_{n}(z) P_{b n}{ }^{2}\left(k_{x}, \alpha_{n}(z), z\right) d z=\right.
\end{aligned}
$$

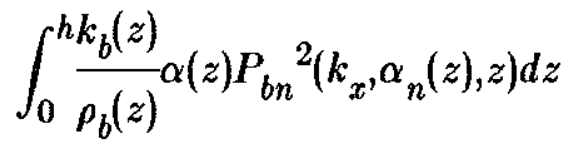

The iteration method described is similar to the procedure followed for solving nonlinear equations by the Newton method. Convergence of this scheme is guaranteed if the initial guess is close to the actual value. Also the convergence is quadratic. These results have been proved in the literature[3].

Another approach is an iteration scheme based on higher order perturbation theory. The unknown function $\alpha(\mathrm{z})$ is expressed as a power series in terms of the 
small parameter $c$ as indicated below.

$$
\alpha(z)=\epsilon a_{0}(z)+c^{2} a_{1}(z)+\cdots+
$$

We now successively determine $\alpha_{0}, \alpha_{1}$, and so on. The convergence of this scheme was found to be slow compared to the earlier method.

\subsection{Reconstruction of the attenuation profile}

We, now, discuss in detail the reconstruction of the attenuation profile assuming that the true value of the sound speed and the density in the sediment layer are known.

To test the inversion method we use synthetic data. This is obtained by solving the forward problem and obtaining the plane wave reflection coefficient for a set of angles given the ocean bottom model. The data that will be used for inversion are, therefore, error free.

\subsubsection{The regularization scheme}

Out of the various methods described in Chapter 6 we use the regularisation method. We assume that the solution is in some sense smooth. Experimental results available on the variation of the attenuation with depth support this assumption. We, therefore, look for a solution with the least structure. The smoothness criterion is defined as beliw: 4 ?

$$
S(\alpha(z))=\int_{0}^{h}\left\{\frac{d^{2} a(z)}{d z^{2}}\right\}^{2} d z
$$

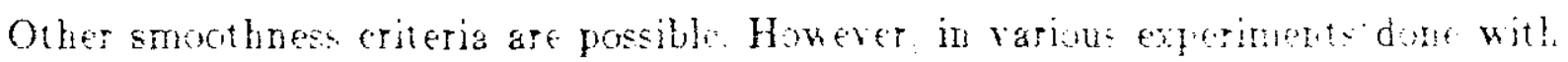


synthetic data, this measure has been found to be adequate.

We start with the integral equation derived in Chapter 5 for the reconstruction of the attenuation profile. Since the reflection coefficient is known only for discrete angular values, the above equation is written as

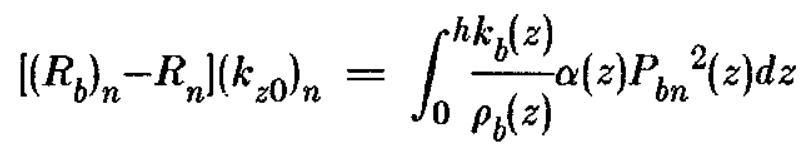

where $\mathbf{n}$ corresponds to the discrete angular values at which the reflection cefficents are determined. The integral equation (7.17) is now written in the following form

$$
d_{n}=\int_{0}^{h} \alpha(z) G_{n}(z) d z
$$

where

$$
\begin{gathered}
d_{n}=\left[\left(R_{b}\right)_{n}-R_{n}\right]\left(k_{z 0}\right)_{n} \\
G_{n}(z)=P_{b n}{ }^{2}(z) \frac{k_{b}(z)}{\rho_{b}(z)}
\end{gathered}
$$

The integral on the right hand side is then written as a sum using a quadrature scheme. We have used the simple Simpsons rule for this purpose. With the sediment modelled as a stack of homogeneous layers the term $P_{b}^{2}\left(k_{x}, z\right)$ in the integrand of the integral equation has the following form in each layer.

$$
\begin{aligned}
P_{b}^{2}\left(k_{x}, z\right)=\left\{A\left(k_{x}\right) \exp \left(i\left(k_{b}^{2}-k_{x}^{2}\right)^{1 / 2} z\right)+\right. \\
\left.B\left(k_{x}\right) \exp \left(-i\left(k_{b}^{2}-k_{x}^{2}\right)^{1 / 2} z\right)\right\}^{2}
\end{aligned}
$$

Therefore, for the degradation resulting from converting the integral into a discrete sum to be small, we must sample at points arrived at by applying the 
Nyquist criterion as the integral equation looks like a Fourier transform. Since the vertical wavenumber varies with depth and the angle of incidence, we will take the maximum value of the wavenumber as the water wavenumber. Based on this the sampling interval must be less than $\lambda_{0} / 4$ where $\lambda_{0}$ corresponds to the wavenumber in the water column.

The integral equation is now written as a discrete sum

$$
d_{i}=\sum_{j} w_{i j} G_{i j} \alpha_{j}
$$

where $w_{i j}$ is the weighting associated with the quadrature scheme. For a set of observations the equation is put in matrix form,

$$
\mathbf{d}=\mathbf{G} \alpha
$$

where the weights $w_{i j}$ have been absorbed in G. The smoothness measure in equation (7.16) is also put in discrete form as shown below.

$$
S(\alpha(z))=\sum_{i}\left(\alpha_{i+1}-2 \alpha_{i}+\alpha_{i-1}\right)^{2}
$$

This is a quadratic measure and $S(\alpha(z))$ is therefore written as

$$
S(\alpha)=\alpha^{T} \mathbf{H} \alpha
$$

The matrix $\mathrm{H}$ is given below.

$$
H=\left[\begin{array}{llllll}
1 & -2 & 1 & . & \cdot & . \\
-2 & 5 & -4 & 1 & \cdot & \cdot \\
1 & -4 & 6 & -4 & 1 & . \\
\cdot & 1 & -4 & 6 & -4 & 1 \\
\cdot & \cdot & \cdot & \cdot & \cdot & \cdot
\end{array}\right]
$$

The problem is now restated as follows. Find a $\alpha$ that minimises $S(\alpha)$ subject 
to $|\mathbf{d}-\mathbf{G m}|^{2}$ being less than $\mathbf{E}^{2}$ (say), the errors associated with the numerical estimation of data. Using $\lambda$ as the Lagrange multiplier we seek to minimise

$$
\alpha^{T} \mathbf{H} \alpha+1 / \lambda\left\{(\mathbf{d}-\mathbf{G} \alpha)^{T}(\mathrm{~d}-\mathbf{G} \alpha)\right\}
$$

Differentiating with respect to each element of $\alpha$ and equating it to zero, we obtain

$$
\begin{aligned}
& \left(\mathbf{G}^{2} \mathbf{G}+\lambda \mathbf{H}\right) \alpha=\mathbf{G}^{T} \mathbf{d} \\
& \alpha=\left(\mathbf{G}^{T} \mathbf{G}+\lambda \mathbf{H}\right)^{-1} \mathbf{G}^{T_{\mathbf{d}}}
\end{aligned}
$$

In some instances it may be necessary to add additional constraints[5] such as a minimum norm criterion in which case the equation to be solved will be as shown below.

$$
\left(\mathbf{G}^{T} \mathbf{G}+\lambda_{1} \mathbf{H}+\lambda_{2} \mathbf{I}\right) \alpha=\mathbf{G}^{T} \mathbf{d}
$$

where $\lambda_{1}$ and $\lambda_{2}$ are two Lagrange multipliers and $I$ is an Identity matrix. In the examples considered in this dissertation, situations needing such additional constraints did not occur.

By using the method described, a smooth solution is obtained. It may be noted that the inversion using generalised inverse eliminates the instability by discarding the small eigenvalues. The small eigenvalues are the ones that contribute to high oscillations in the solution and eliminating the small eigenvalues is equivalent to a low pass filtering operation on the solution to get a smoothed version. The regularisation method also overcomes the instability by assuming that the solution is in some sense smooth and in this context both the methods are analogous.

Having obtained a solution it may be seen that there are sharp increases/ 
decreases in some region indicating that the assumption the the solution is smooth over the entire region is not correct. The fact that such sharp changes are seen in the reconstructed profile even after making the smoothness assumption is a strong indication that these features are not an artifact of the inversion scheme. We will, therefore, be justified in making the assumption that sharp changes do occur at these depths and hence look for smooth solution in the intervening depths. An example of this will be presented later on.

The Lagrange multiplier $\lambda$ determines the smoothness of the solution. The larger the value of $\lambda$ the smoother the solution. This means that larger values of $\lambda$ will lead to poorer resolution. For lower values of $\lambda$ more structure will be observed but at the same time the variance of the solution will increase. One approach in determining $\lambda$ is to initially take large value and reduce it with each iteration as suggested by Marquadt[6]. At each iteration the residual is computed and the iteration procedure terminated when any one of the following conditions are met.

$$
\begin{aligned}
& |\mathbf{d}-\mathbf{G} \alpha|^{2} \leq E^{2} \\
& \left|\alpha_{n+1}-\alpha_{n}\right|^{2} \leq e^{2}
\end{aligned}
$$

Another method of determining the Lagrange multiplier is based on some prior knowledge of the upper bound for the noise power in the data and some measure of the smoothness desired in the solution[7]. We write the equation to be solved in the following form

$$
\mathbf{G} \alpha=\mathbf{d}+\mathbf{z}
$$

where $\mathrm{z}$ is the vector containing the error. If $\mathrm{E}^{2}$ is the upper bound for noise power, then 


$$
|\mathbf{d}-\mathbf{G} \alpha|^{2} \leq E^{2}
$$

The smoothness measure is obtained from equation (7.22) reproduced below.

$$
S(\alpha(z))=\sum_{i}\left(\alpha_{i+1}-2 \alpha_{i}+\alpha_{i-1}\right)^{2}
$$

If we can have a measure for this we write

$$
\alpha^{T} \mathbf{H} \alpha \leq \epsilon^{2}
$$

where $\epsilon^{2}$ is the measure of smoothness. Combining equations (7.32) and (7.33) we obtain

$$
|\mathrm{G} \alpha-\mathrm{d}|^{2}+\frac{E^{2}}{\epsilon^{2}} \alpha^{T} \mathbf{H} \alpha \leq 2 E^{2}
$$

We now find a solution for $\alpha$ that minimises the left hand side. The solution is obtained in a manner similar to that given earlier.

$$
\left(\mathbf{G}^{T} \mathbf{G}+\lambda \mathbf{H}\right) \alpha=\mathbf{G d}
$$

where,

$$
\lambda=E^{2} / \epsilon^{2}
$$

The factor $\mathbf{E}^{2} / \epsilon^{2}$ is a measure of how smooth the solution will be. When $\epsilon^{2}$ is small $\mathrm{E}^{2} / \epsilon^{2}$ will be large and give a smooth solution. By making a priori assumptions about $\mathrm{E}^{2}$ and $\epsilon^{2}$ we obtain the Lagrange multiplier $\lambda$. 


\subsubsection{Angular aperture for data points}

In Chapter 5 we obtained the condition for the applicability of the Born approximation. The condition to be met is given below.

$$
\left\|k_{b}\right\|\|\alpha\| \| G\left(z, z^{\prime} \mid\right)_{\max } h \ll 1
$$

The Green's function in the above equation is for the assumed background model. For any arbitrary sound speed and density profile the Green's function can be obtained using the uniform asymptotic solution for the wave equation. This solution is, however, valid only when these parameters vary slowly with depth and increase monotonically with depth, a situation that is met in marine sediments. The solution for the Green's function is given in Appendix A.

The Green's function is a function of horizontal wavenumber as well as the spatial parameter $z$ and $z$, points representing source and receiver positions. If the Green's function is expressed as function of the horizontal wavenumber there are regions in the horizontal wavenumber domain where the Green's function assumes large values. In marine sediment the sound speed at the sediment water interface is, in some instances, less than its value in the water column. The sound speed then increases monotonically with depth with a possible discontinuity at the sedimentsubbottom interface. With this structure, the sediment layer behaves like a wave guide and complete trapping of energy is possible. There will, therefore, be values of horizontal wavenumber for which the Green's function takes very large values.

If we are to remain within the region of applicability of the Born approximation we will be best served by remaining in the region of angles where the Green's function has low values. This will permit the inequality in equation 


$p=1.0 \mathrm{~g} / \mathrm{cm}^{3} \quad$ Sound Speed $C_{2}=1800 \mathrm{~m} / \mathrm{sec} \quad$ Sub. bottom

Figure 7-1: Simplified ocean bottom model to study behaviour of Green's function 


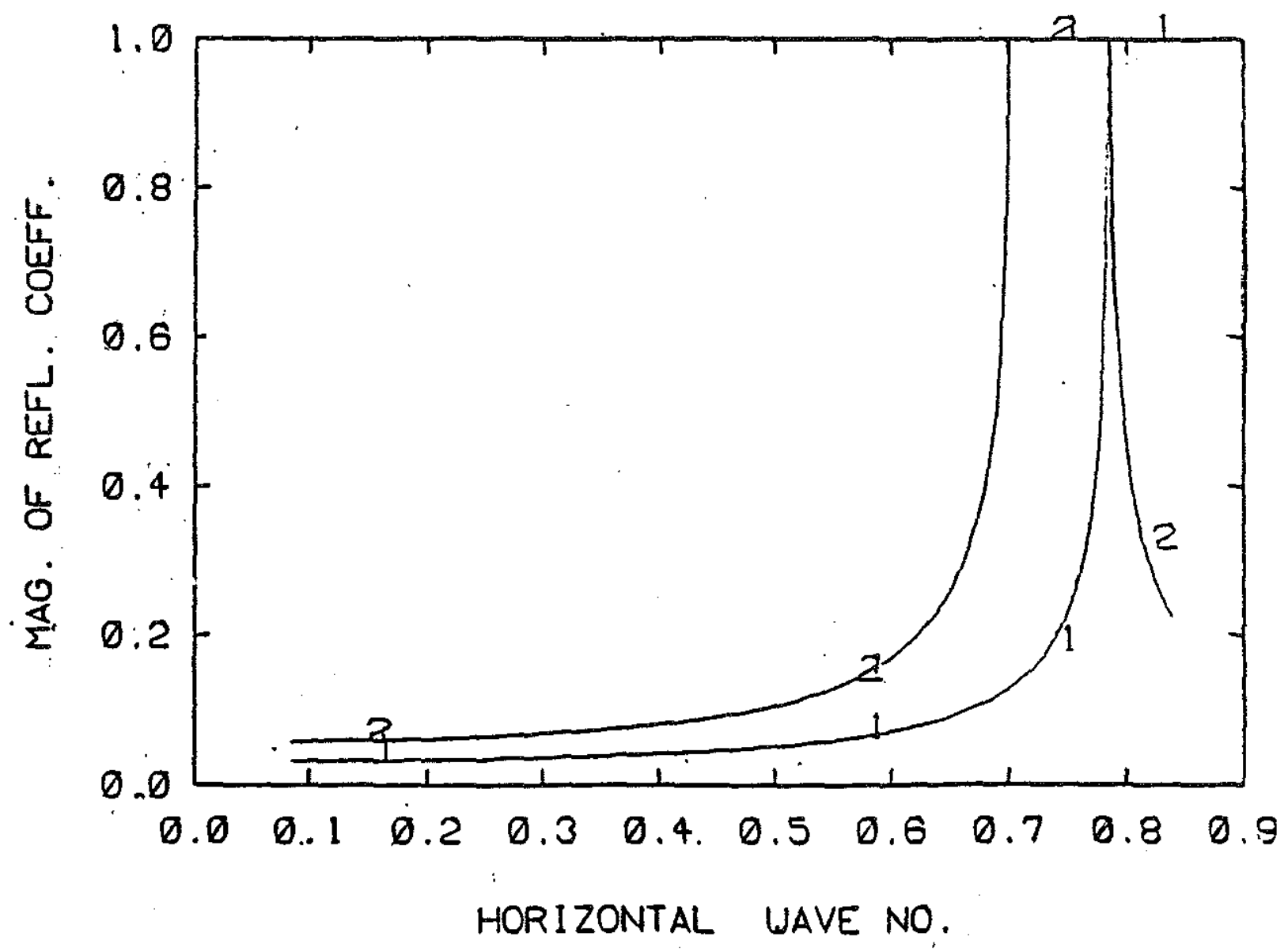

Figure 7-2: Magnitude of Rayleigh reflecion coefficent at the two interfaces 1-water/sediment interface, 2-sediment/subbottom interface 
(7.38) to be satisfied for larger values of the pertubation and sediment thickness. Since the background model parameters are known numerical evaluation of the Green's function can be performed. From these computations we choose the angular aperture where the Green's function has low values.

A qualitative feeling for the acceptable range of values of horizontal wavenumber can be obtained by studying the simple model in Figure 7-1. For this model there are three distinct angular region, or equivalently, three ranges of horizontal wavenumbers that we will consider. These are $0<k_{x}<k_{2}$, $k_{2}<k_{x}<k_{1}$, and $k_{1}<k_{x}<k_{0}$. For the first range, there are no angles at which total reflection at the interfaces occurs. Energy is transmitted into the water column and the subbottom. In the second region total reflection occurs at the bottom interface but energy is transmitted into the water column. In the region where the horizontal wavenumber is greater than $k_{1}$, there is total reflection at the water sediment interface. However, the magnitude of the reflection coefficient at the sediment/subbottom interface is no longer unity. Therefore complete trapping is not possible for this particular model. However, there is a region of horizontal wavenumber where the reflection coefficient magnitude for both the interfaces is close to unity. Near trapping of energy can take place in this region and the Green's fuction can assume large values. Figure 7-2 is a plot of the magnitude of the Rayleigh reflection coefficient at the two interfaces for this model. The region where both these reflection coefficients come close to unity approximately lies in the range of horizontal wavenumbers from 0.76 to 0.785 .

For the model in Figure 7-1, the Green's function is given by the following expression. 


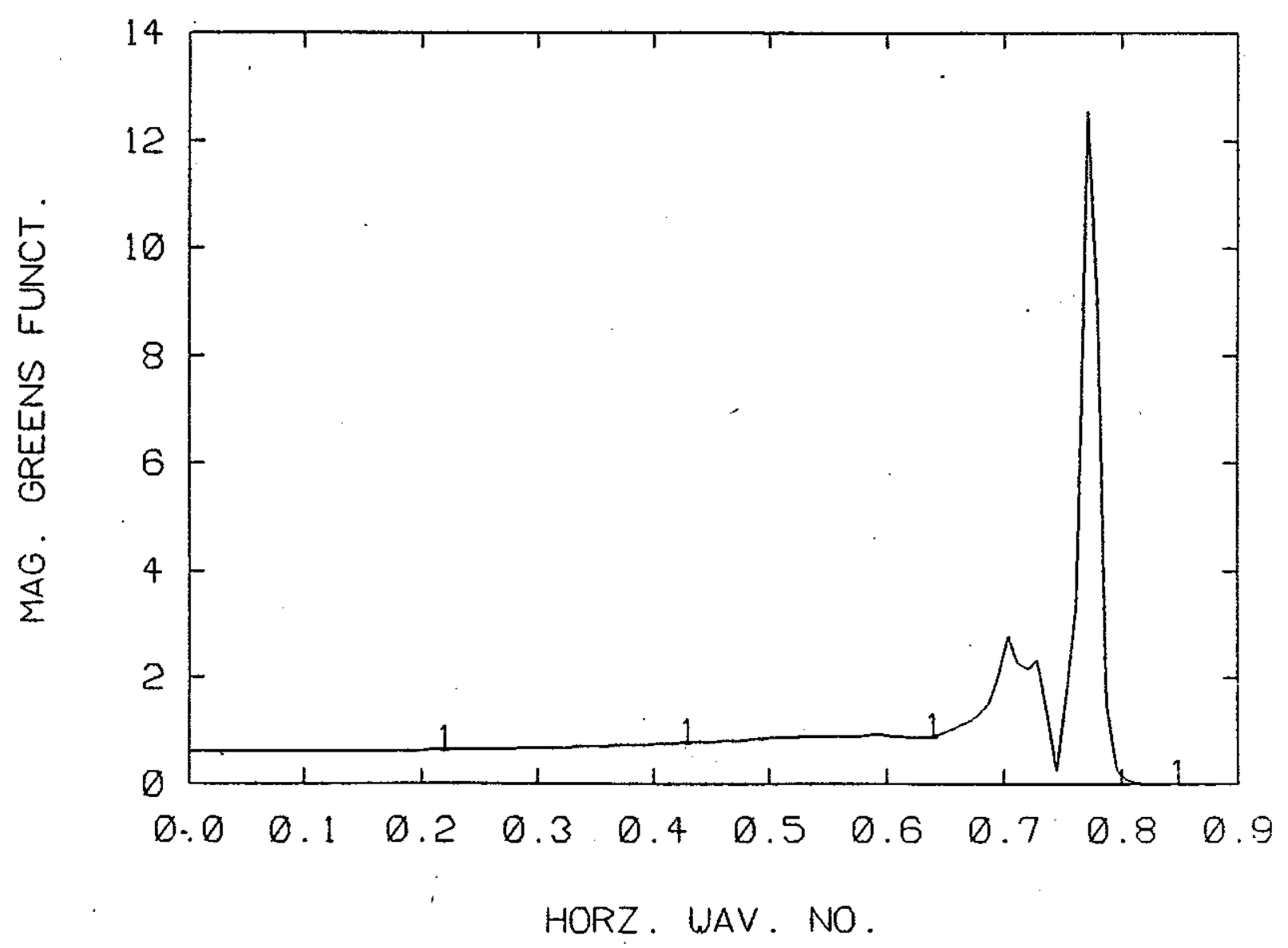

Figure 7-3: Magnitude of Green's function as a function of horizontal wavenumber, Frequency $=200 \mathrm{~Hz}$

$$
\text { Source depth }=30 \mathrm{~m} \text {, Receiver depth }=10 \mathrm{~m}
$$




$$
\begin{aligned}
& G\left(z, z^{\prime}\right)=\frac{\left[R_{s} e^{i k_{z 1} z}+e^{-i k_{z 1} z}\right]\left[e^{i k_{z 1} z^{\prime}}+R_{b} e^{-i k_{z 1} z^{\prime}+2 i k_{z 1} h}\right]}{2 i k_{z 1}\left(1-R_{s} R_{b} e^{2 i k} z 1^{h}\right)} \\
& 0 \leq z \leq z^{\prime}
\end{aligned}
$$

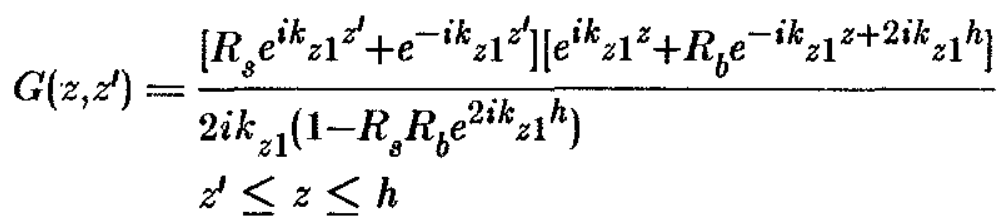

The plane wave reflection coefficients $R_{s}$ and $R_{b}$ are given by the followin equations.

$$
\begin{aligned}
& R_{s}=\frac{k_{z 1}-k_{z 0}}{k_{z 1}+k_{z 0}} \\
& R_{b}=\frac{k_{z 1}-k_{z 2}}{k_{z 1}+k_{z 2}}
\end{aligned}
$$

with,

$$
\begin{aligned}
& k_{z 0}=\left[k_{0}{ }^{2}-k_{x}{ }^{2}\right]^{1 / 2}, k_{0}=\frac{\omega}{C_{0}} \\
& k_{z 1}=\left[k_{1}{ }^{2}-k_{x}{ }^{2}\right]^{1 / 2}, k_{1}=\frac{\omega}{C_{1}} \\
& k_{z 2}=\left[k_{2}{ }^{2}-k_{x}{ }^{2}\right]^{1 / 2}, k_{2}=\frac{\omega}{C_{2}}
\end{aligned}
$$

Our earlier discussion indicates that though there are no poles on the real axis, off axis poles in the complex domain of horizontal wave number may exist in the range of horizontal wavenumbers $k_{2}<k_{x}<k_{1}$. The effect of these off axis poles are felt as we move along the real axis. There are therefore values of horizontal wavenumber for which the Green's function can take large values. 
The magnitude of the Green's function in equation (7.39) is plotted in Figure 7-3 as a function of the horizontal wave number. The source and receiver positions are $30 \mathrm{~m}$ and $10 \mathrm{~m}$ respectively from the water/sediment interface. The acoustic parameters used in the computation are indicated in Figure 7-1.It is seen that the magnitude of the Green's function has large values at positions where the magnitude of the plane wave reflection coefficients at the two interfaces are close to unity as anticipated earlier. The figure shows also that in the region where there is least trapping, i.e. $0<k_{x}<k_{2}$, the Green's function has the least value. This corresponds to pre-critical angles. In the second region, the behaviour of the Green's function becomes complex and there are values of horizontal wavenumber where the magnitude of the Green's fuction becomes large. When the waves in the sediment becomes inhomogeneous the magnitude of the Green's function decreases. The advantage of using the information from the pre-critical range of angles in the inverse problem is therefore obvious. Rays incident at pre-critical angles will probe deep into the sediment whilst rays incident at angles above the critical angle will turn within the sediment layer there will be turning points in the sediment and therefore these rays will have no information about medium beyond the turning depth. If the entire range of angles from grazing to normal incidence is used there may be certain angular regions where the Born approximation is not valid and this can lead to degradation in the reconstructed profiles. However, since an iterative scheme is employed the magnitude of the perturbation gets reduced at each stage of iteration and the scheme may converge unless the non-linearity is too strong.

Only rays in the precritical region probe the entire depth and the Green's function has the lowest magnitudes in this range. We have, therefore, used only this range of angles in the reconstruction of the attenuation profiles. 


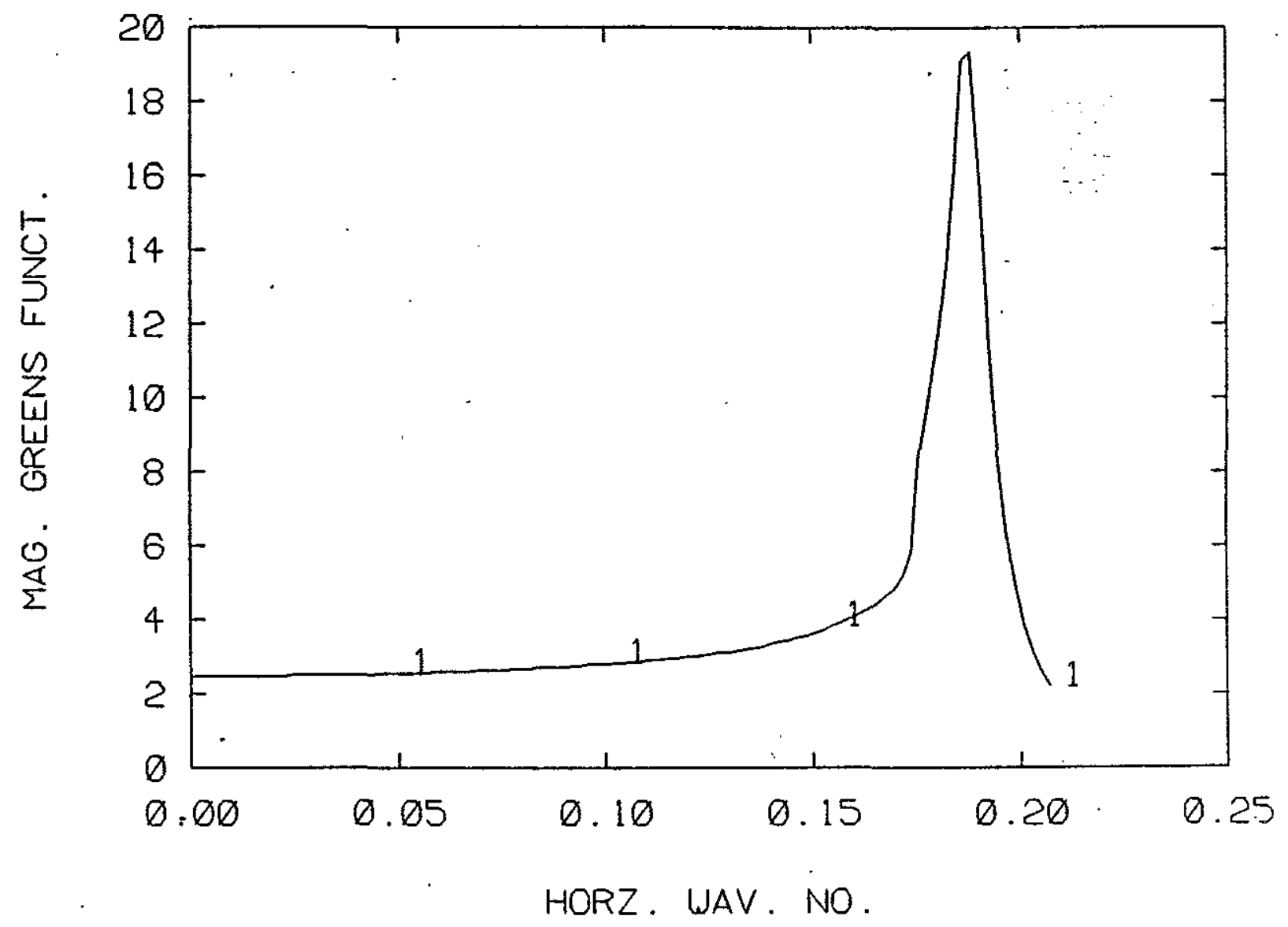

Figure 7-4: Magnitude of Green's function as function of horizontal wavenumber, Frequency $=50.0 \mathrm{~Hz}$

Source depth $=30 \mathrm{~m}$, Receiver depth $=10 \mathrm{~m}$ 


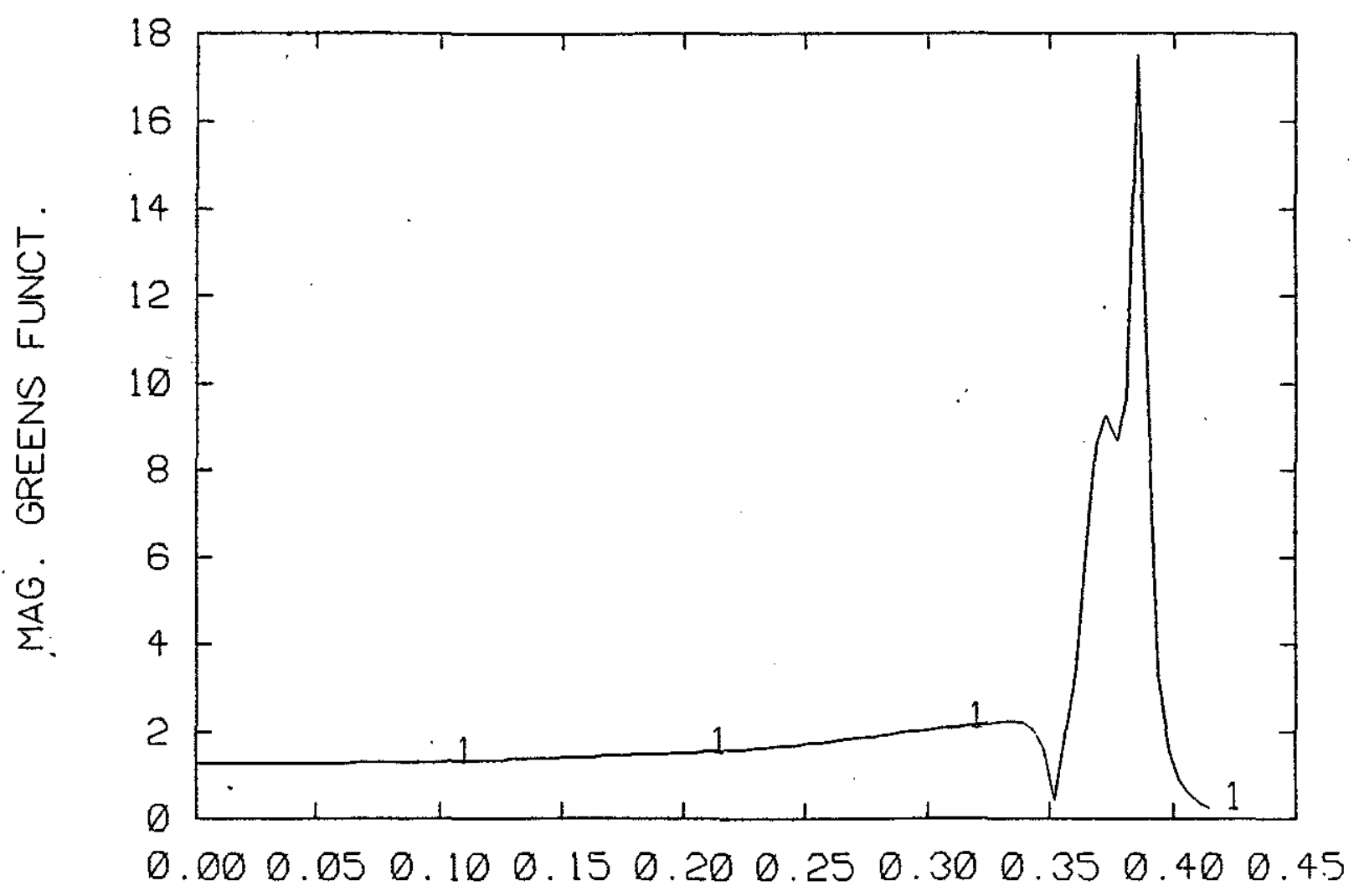

HORZ. WAV. NO.

Figure 7-5: Magnitude of Green's function as a function of horizontal wavenumber, F requency $=100.0 \mathrm{~Hz}$

Source depth $=30 \mathrm{~m}$, Receiver depth $=10 \mathrm{~m}$ 


\subsection{Effect of experimental and geoacoustic parameters}

\subsubsection{Frequency}

Figures 7-3 to 7-5 show the magnitude of the Green's function vs horizontal wavenumber at different frequencies. In the pre-critical region, a rough $1 / \omega$ dependence is seen. This result is not surprising because in this region the effect of the interfaces in trapping energy in least and the Green's function behaves very much like the free-space Green's function. For homogeneous medium, the free space Green's function behaves as $1 / k_{z}$ where $k_{z}$ is the vertical wavenumber. This is equivalent to $1 / \omega$ dependence. In Appendix A the expression for Green's function in terms of the uniform asymptotic solution has been obtained for an arbitrary variation of sound speed and density. In the pre-critical range of angles, there are no turning points in the sediment layer and the uniform asymptotic solution reduces to WKB solution as shown in the appendix and the Green's function has an approximate $1 / \omega$ dependence.

Earlier for the applicability of the Born approximation we noted that the inequality to be satisfied is,

$$
\left\|\frac{\omega}{C_{b}(z)}\right\|\|\alpha\| h\left|G\left(z, z^{\prime}\right)\right|_{\max } \ll 1
$$

We have shown that in the pre-critical angles the magnitude of the Green's function has an approximate $1 / \omega$ dependence. From this alone, it appears that a higher frequency is better since the magnitude of the Green's function is small. However, the magnitude of the perturbation $k_{b}(z) \alpha(z)$ is directly propotional to frequency. Therefore its product with the Green's function is approximately constant. We conclude, that the operating frequency will not have significant effect 
$-152-$

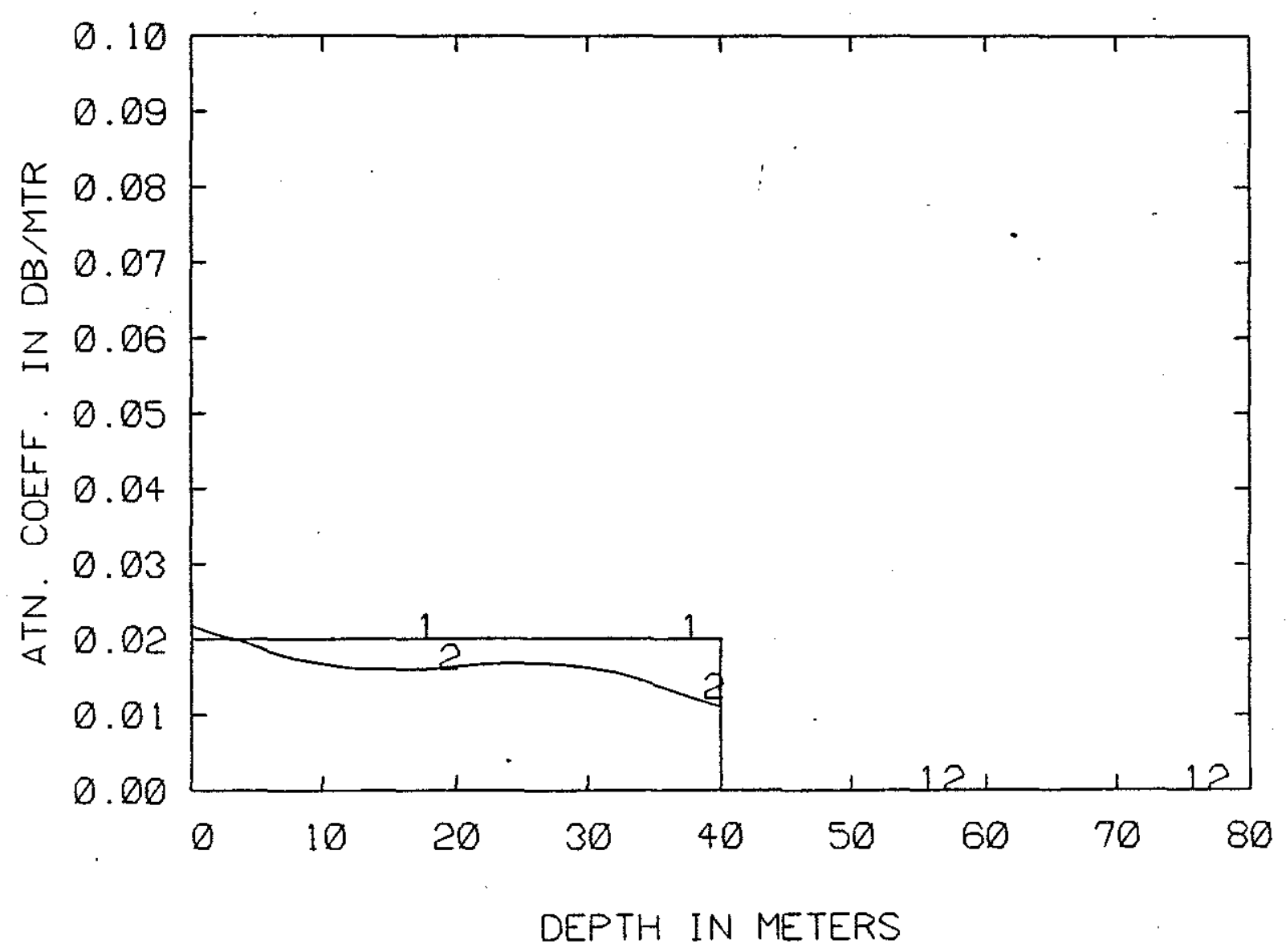

Figure 7-6: Reconstruction of constant attenuation of $0.02 \mathrm{~dB} / \mathrm{m}$ in the layer, Frequency $=100 \mathrm{~Hz}$

1 - True profile, 2- Reconstructed profile 


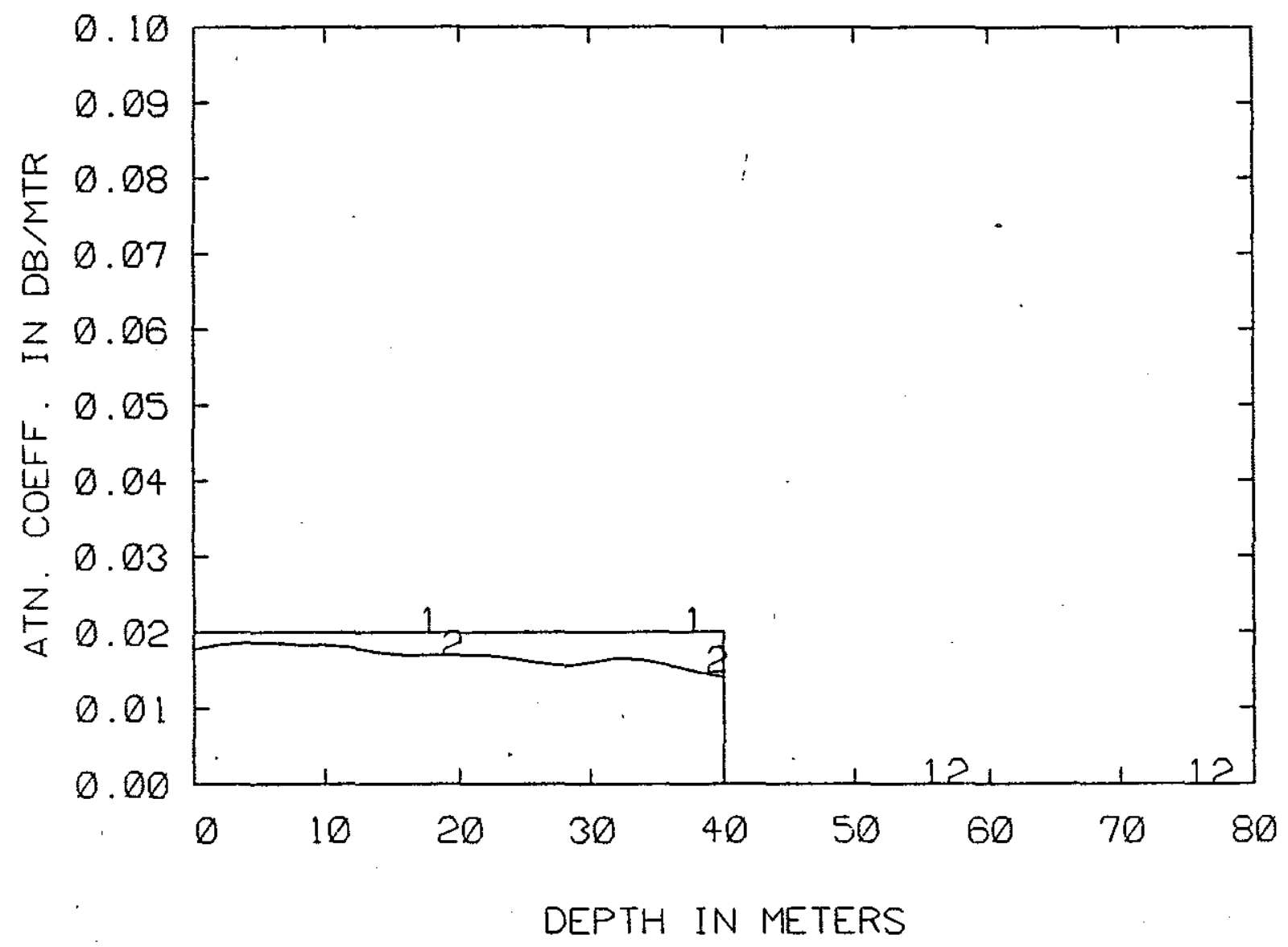

Figure 7-7: Reconstruction of constant attenuation of $0.02 \mathrm{db} / \mathrm{m}$ in the layer, Frequency $=25 \mathrm{~Hz}$

1- True profile, 2- Reconstructed profile 
on reconstruction. To see this, we carry out inversion using synthetic data generated at different frequencies. The result obtained at the first step of the iteration process is at figures 7-6 and 7-7. Though the frequency was increased four fold it did not have any appreciable effect on the reconstructed profile.

\subsubsection{Attenuation coefficient}

For two values of constant attenuation in the sediment $(0.005 \mathrm{~dB} / \mathrm{m}$ and $0.02 \mathrm{~dB} / \mathrm{m}$ ) inversions were carried out. The results are shown in Figures 7-7 and 7-8. As anticipated the larger the perturbation the poorer the reconstruction.

\subsubsection{Depth of sediment}

The magnitude of the Green's function was computed for different sediment layer thicknesses while keeping the other acoustic parameters of the model fixed. The plot of the Green's function magnitude as a function of horizontal wavenumber is shown in Figures 7-9 and 7-10. The Green's function magnitude remains approximately the same for all the three cases in the pre-critical range. Its behaviour beyond this range is however complicated. In equation (7.39) the only place where the sediment thickness enters the expression is in the phase term $\exp \left(2 i k_{z 1} h\right)$. Therfore for small values of $\mathrm{R}_{\mathrm{s}}$ and $\mathrm{R}_{\mathrm{b}}$, which is approximately truc for precritical angles the sediment thickness will have little effect on the magnitude of the Green's function. This is true even in cases where the sound speed and density in the sediment layer varies in an arbitrary manner. We can, therefore, conclude that the sediment thickness has no significant effect on the magnitude of the Green's function in the pre-critical range of angles. On the other hand in the expression in equation (7.38) we note that the region of applicability of the Born approximation is related to the depth of the sediment. We anticipate that with 
$-155-$

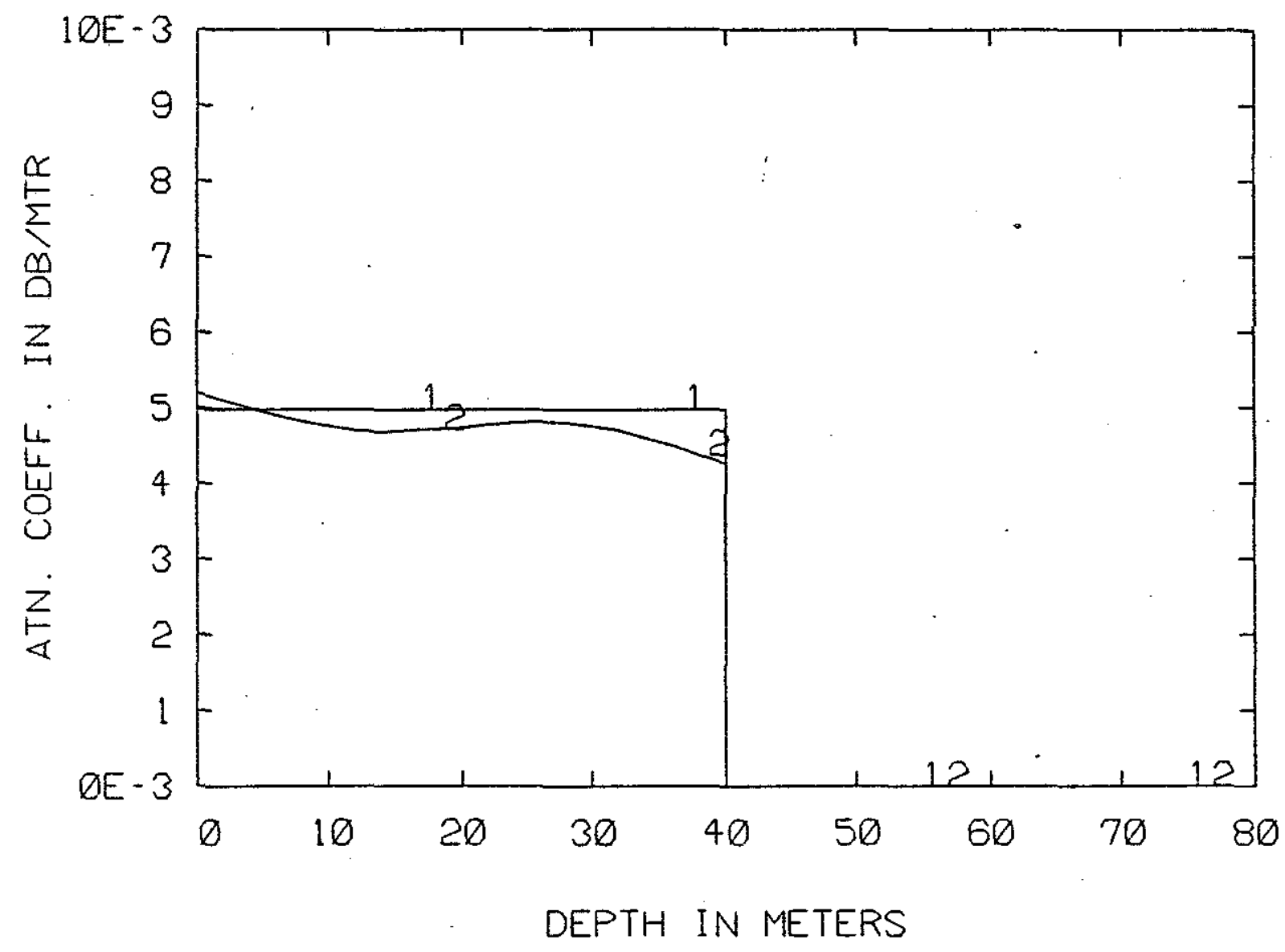

Figure 7-8: Reconstruction of constant attenuation of $0.005 \mathrm{~dB} / \mathrm{m}$ in the layer, Frequency $=25 \mathrm{~Hz}$

1- True profile, 2- Reconstructed profile 
$-156-$

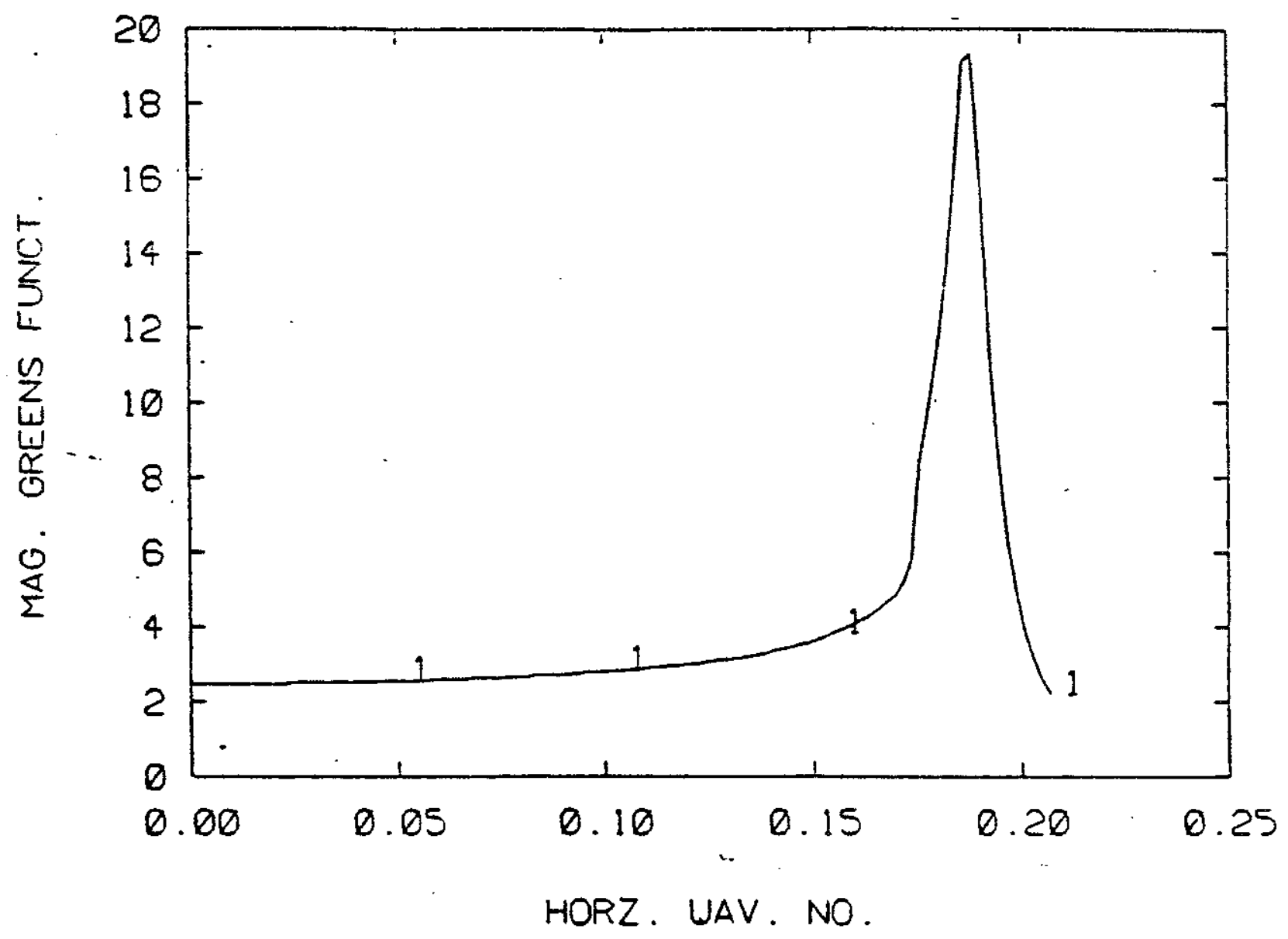

Figure 7-8: Magnitude of Green's function as a function of horizontal wavenumber, Depth of secimes: $=40 \mathrm{~m}$

$$
\text { Frequency }=50 \mathrm{~Hz}
$$


$-157-$

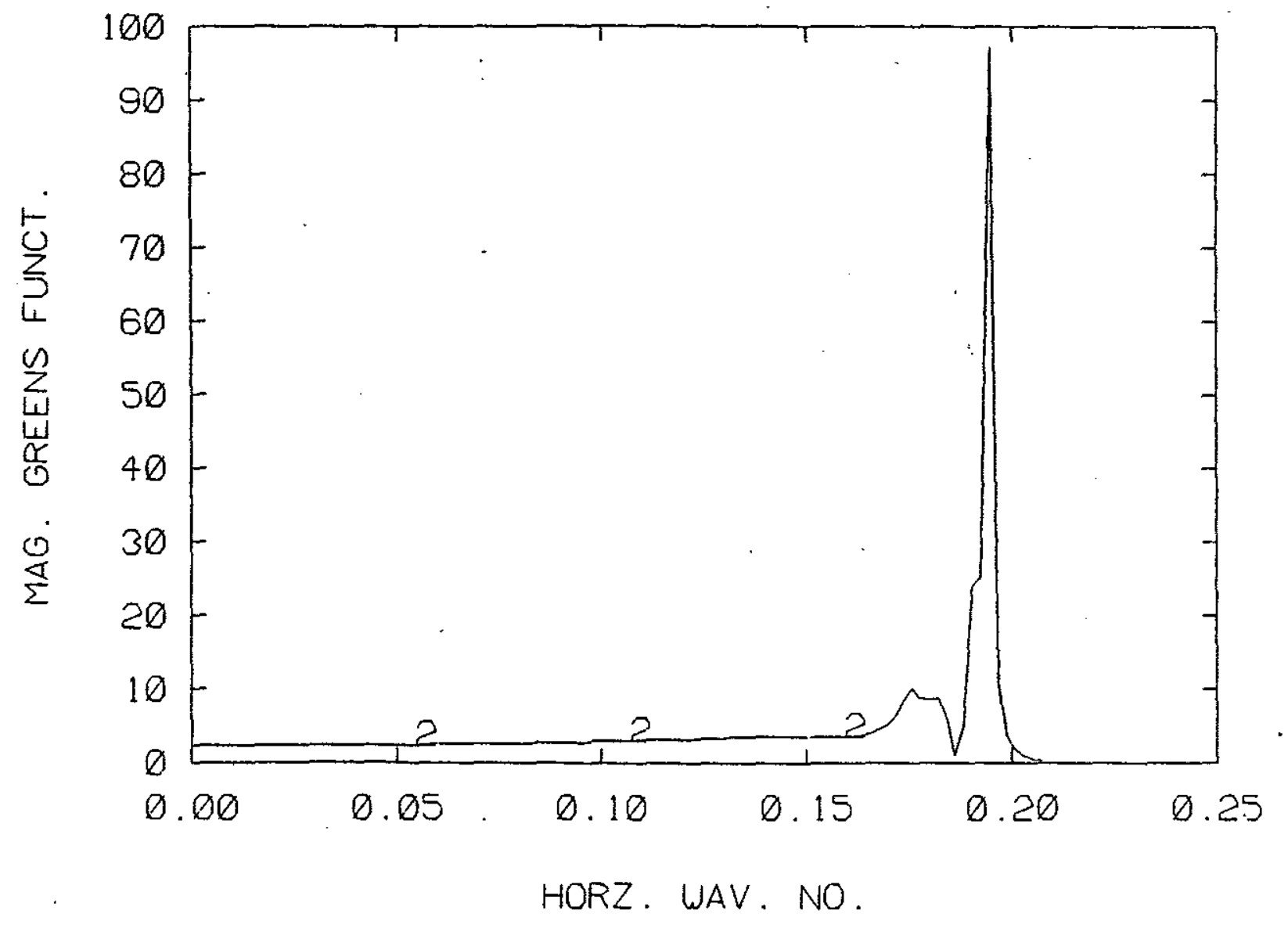

Figure 7-10: Magnitude of Green's function as a function of horizontal Wavenumber, Depth of sediment $=120 \mathrm{~m}$

Frequency $=50 \mathrm{~Hz}$ 
$-158-$

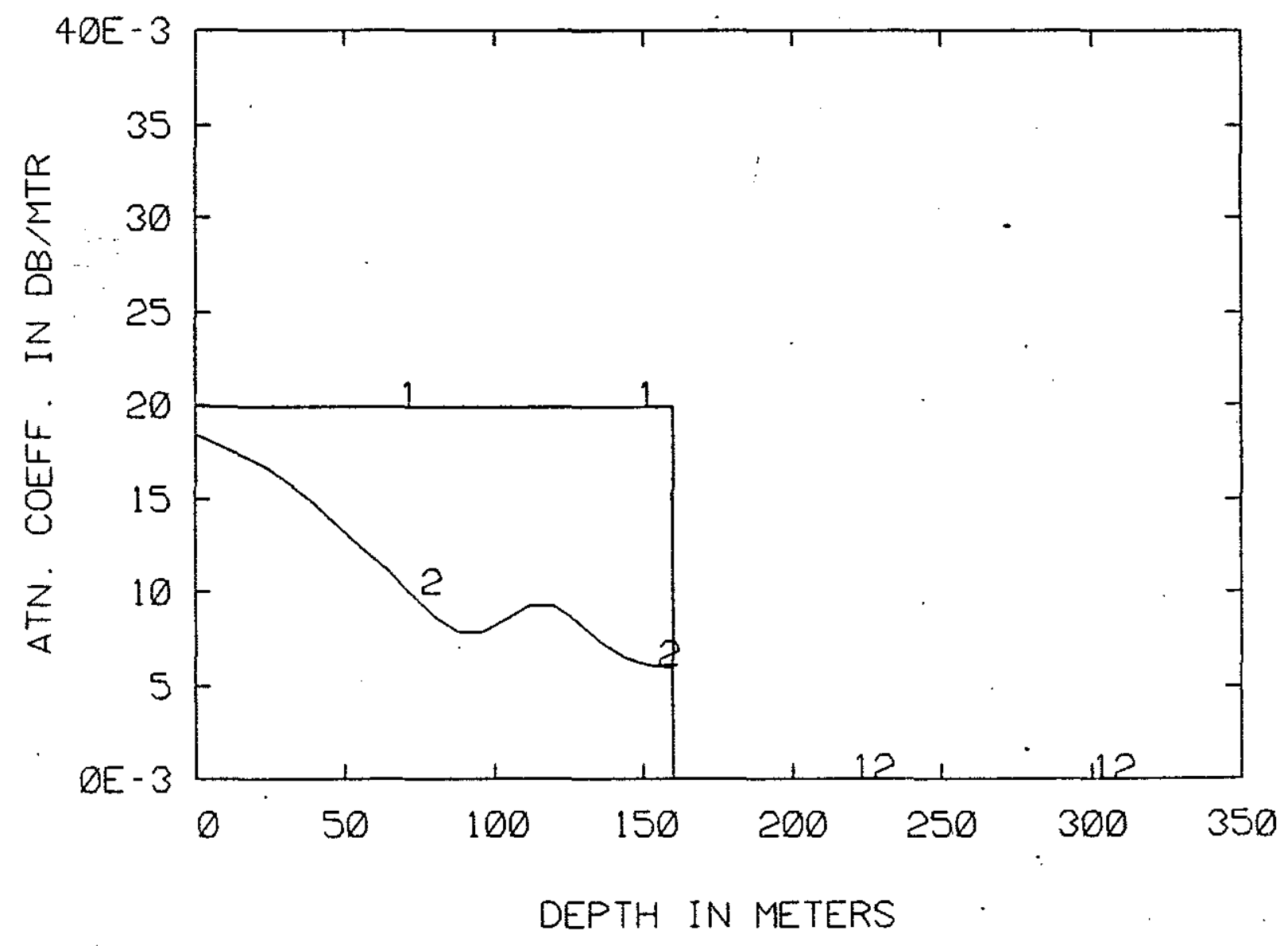

Figure 7-11: Reconstruction of constant attenuation in the layer, layer thickness $=160 \mathrm{~m}$, frequency $=25 \mathrm{~Hz}$ 
increasing depth the errors made in making the Born approximation increases and this will manifest itself in the reconstructed profiles. Figures 7-7 and 7-11 show the reconstructed profiles for different slab thicknesses. We have increased the slab thickness four times leaving every other parameter the same. We note that the increase in thickness has resulted in poorer reconstruction.

\subsubsection{Strength of the discontinuity at the interface}

The strength of the discountinuity at the bottom interfaces was increased by changing the values of the sound speed in the sub-bottom. The magnitude of the Green's function for the two cases are shown in Figure 7-12 and 7-13. There is very little change in the magnitude of the Green's function in the pre-critical range and therefore the strength of the discontinuty is not likely to have any appreciable effect on the reconstructed profiles. However, we note that as the sound speed in the sub-bottom increases the angular range of pre-critical angles decreases and therefore will affect the number of data points that can be used in the inversion scheme.

\subsection{Examples of reconstruction}

We now test the inversion algorithm developed earlier using different type of profiles. The profiles used are given in Figure 7-14. The profile shapes have been chosen so as to cover possible attenuation profiles in marine sediments. The magnitude of the attenuation coefficient chosen falls within the acceptable range of values for marine sediments at the frequency considered.

In each case the input data for the scheme i.e the plane wave reflection coefficient, is computed using the propagator matrix method described in Chapter 


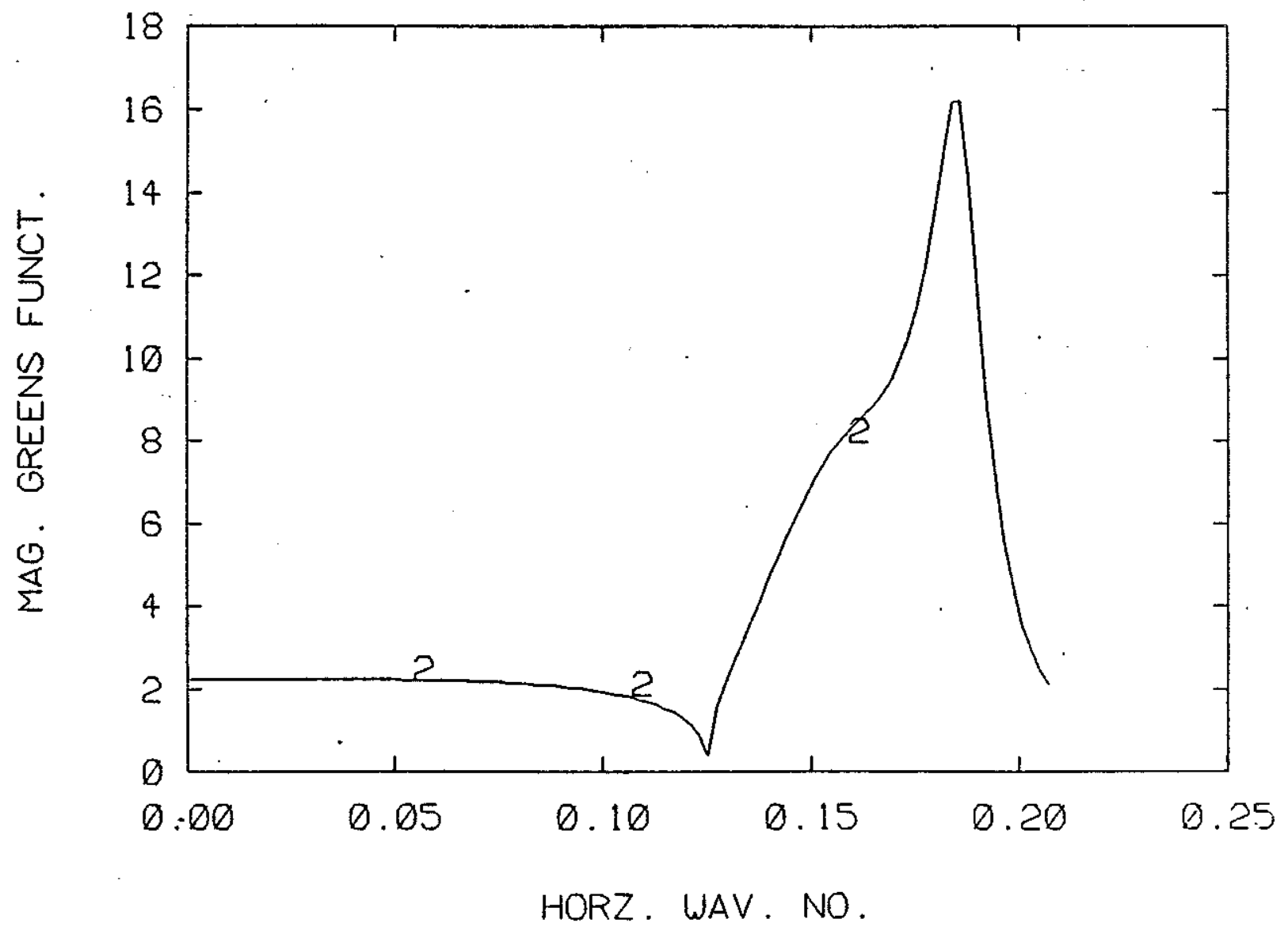

Figure 7-12: Magnitude of Green's function as function of horizontal wavenumber, Sound speed in sub-bottom $=2500 \mathrm{~m} / \mathrm{sec}$ 


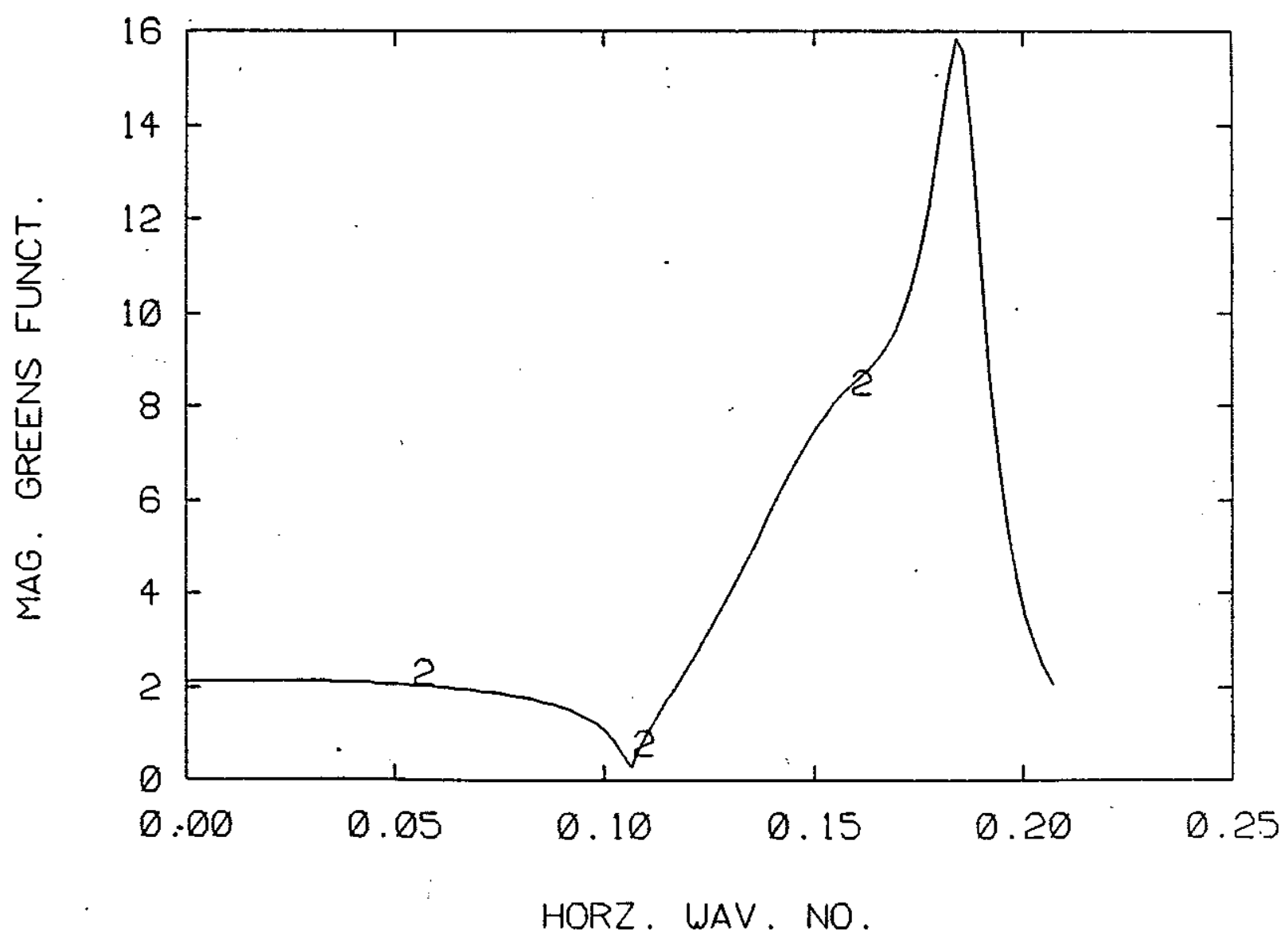

Figure 7-13: Magnitude of Green's function as a function of horizontal wavenumber, Sound speed in sub-bottom $=3000 \mathrm{~m} / \mathrm{sec}$ 


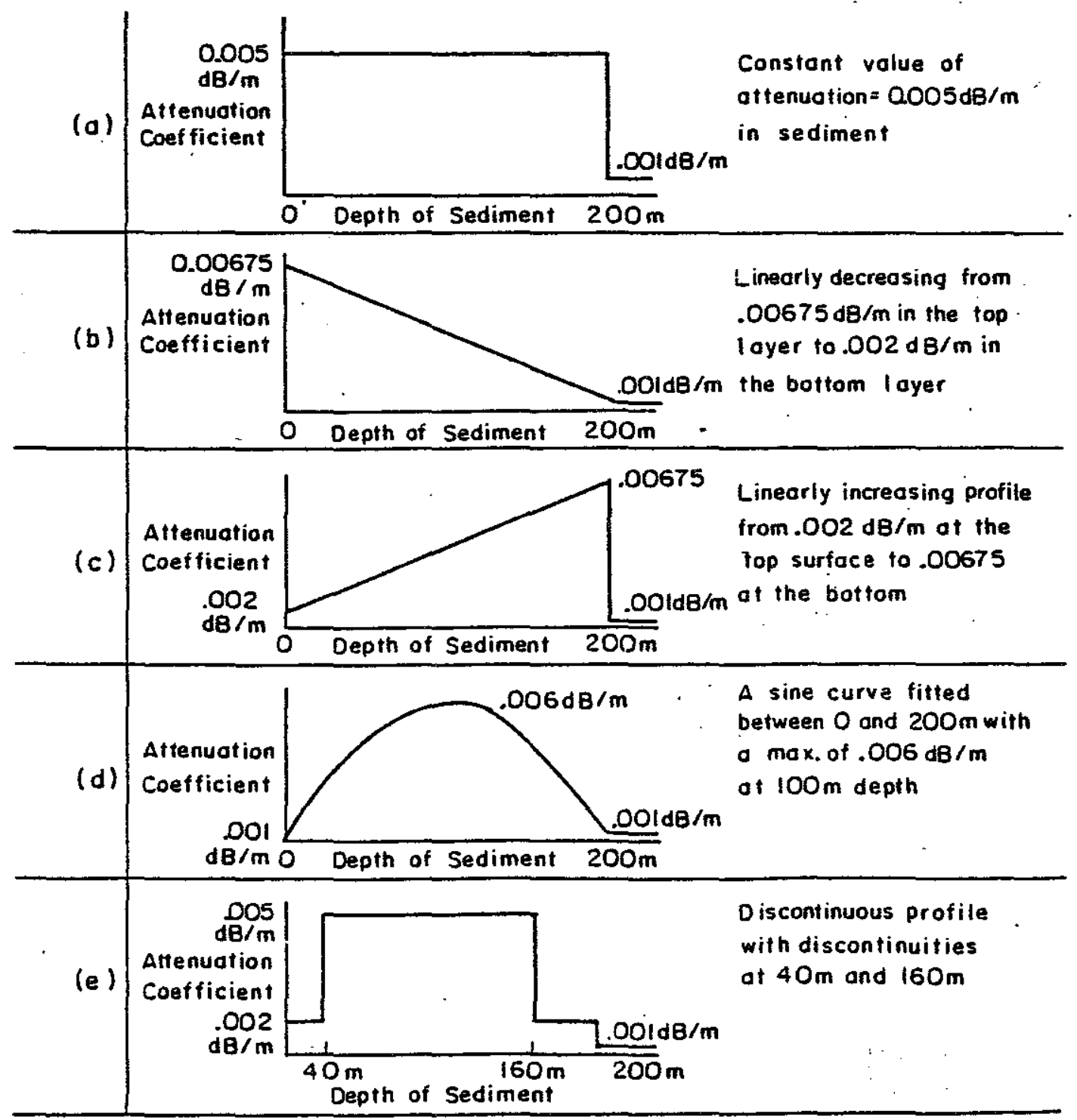

Figure 7-14: Attenuation coefficient profile shapes 
$-163-$

Water Column: Density $=1.0 \mathrm{~g} / \mathrm{cm}^{3}$

Sound Speed $=1500 \mathrm{~m} / \mathrm{sec}$

Attenuation $=0.0$

\begin{tabular}{|c|c|c|c|}
\hline Depth in Meters & Density & Sound Speed M/S & Attenuation \\
\hline 10 & 1.7 & 1517.56 & \multirow{20}{*}{$\begin{array}{l}\text { Various profiles adopted. } \\
\text { These ate shown in figure } 7.1\end{array}$} \\
\hline $20^{\circ}$ & 1.7 & 1530.40 & \\
\hline 30 & 1.7 & 1543.14 & \\
\hline 40 & 1.7 & 1555.74 & \\
\hline 50 & 1.7 & 1568.20 & \\
\hline 60 & 1.7 & 1580.52 & \\
\hline 70 & 1.7 & 1592.69 & \\
\hline 80 & 1.7 & 1604.74 & \\
\hline$\frac{5}{2}$ & 1.7 & 1616.64 & \\
\hline 100 & 1.7 & 1628.41 & \\
\hline 110 & 1.7 & 1640.04 & \\
\hline 120 & 1.7 & 1651.55 & \\
\hline 130 & 1.7 & 1662.92 & \\
\hline 140 & 1.7 & 1674.16 & \\
\hline 150 & 1.7 & 1685.28 & \\
\hline 160 & 1.7 & 1696.27 & \\
\hline 170 & 1.7 & 1707.14 & \\
\hline 180 & 1.7 & 1717.88 & \\
\hline 190 & 1.7 & 1728.50 & \\
\hline 200 & 1.7 & 1739.00 & \\
\hline
\end{tabular}

Table 7-I: Parameters used for ocean bottom model 


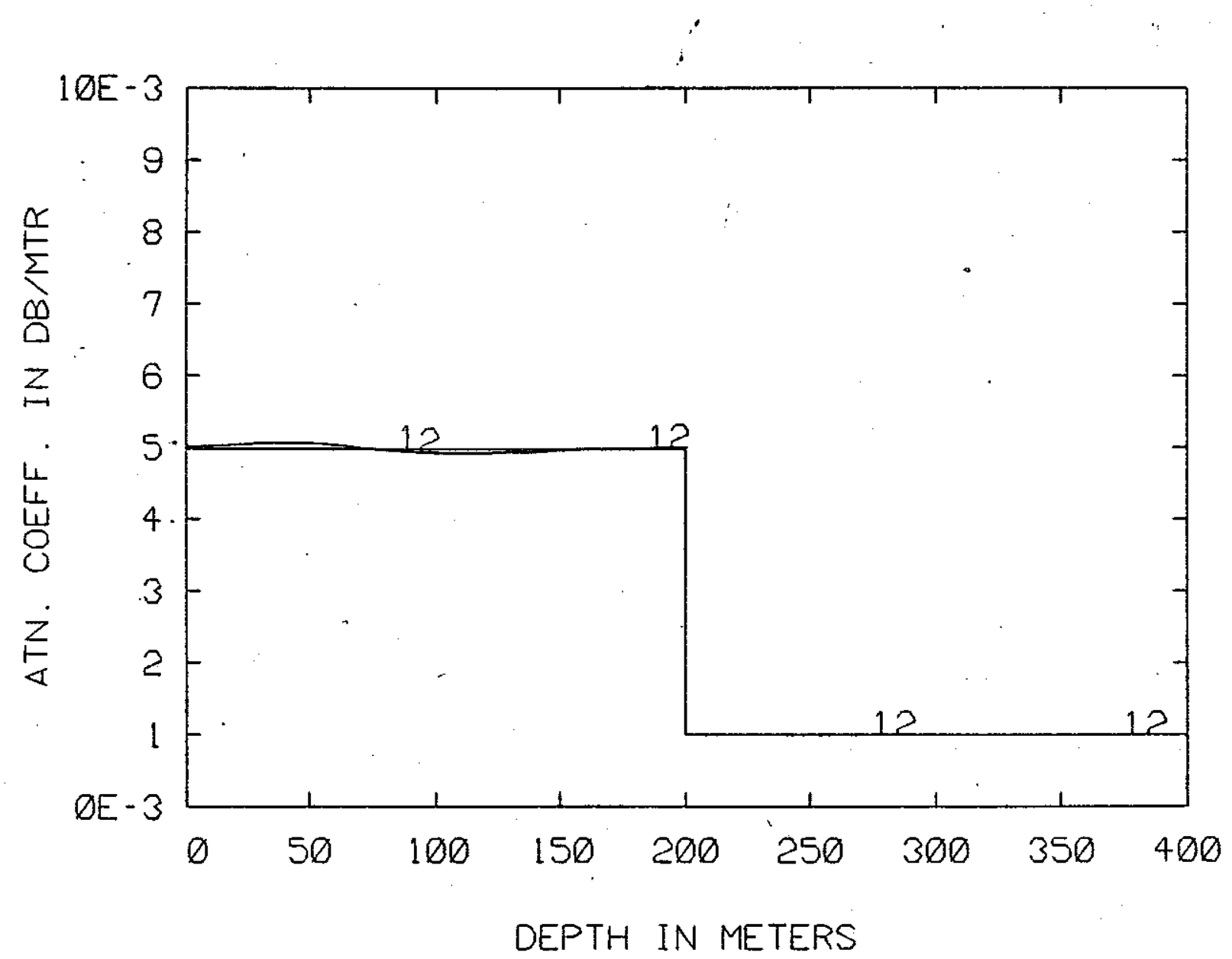

Figure 7-15: Reconstruction of profile in figure 7-14(a)

1- True profile, 2- Reconstructed profile 
$-165-$

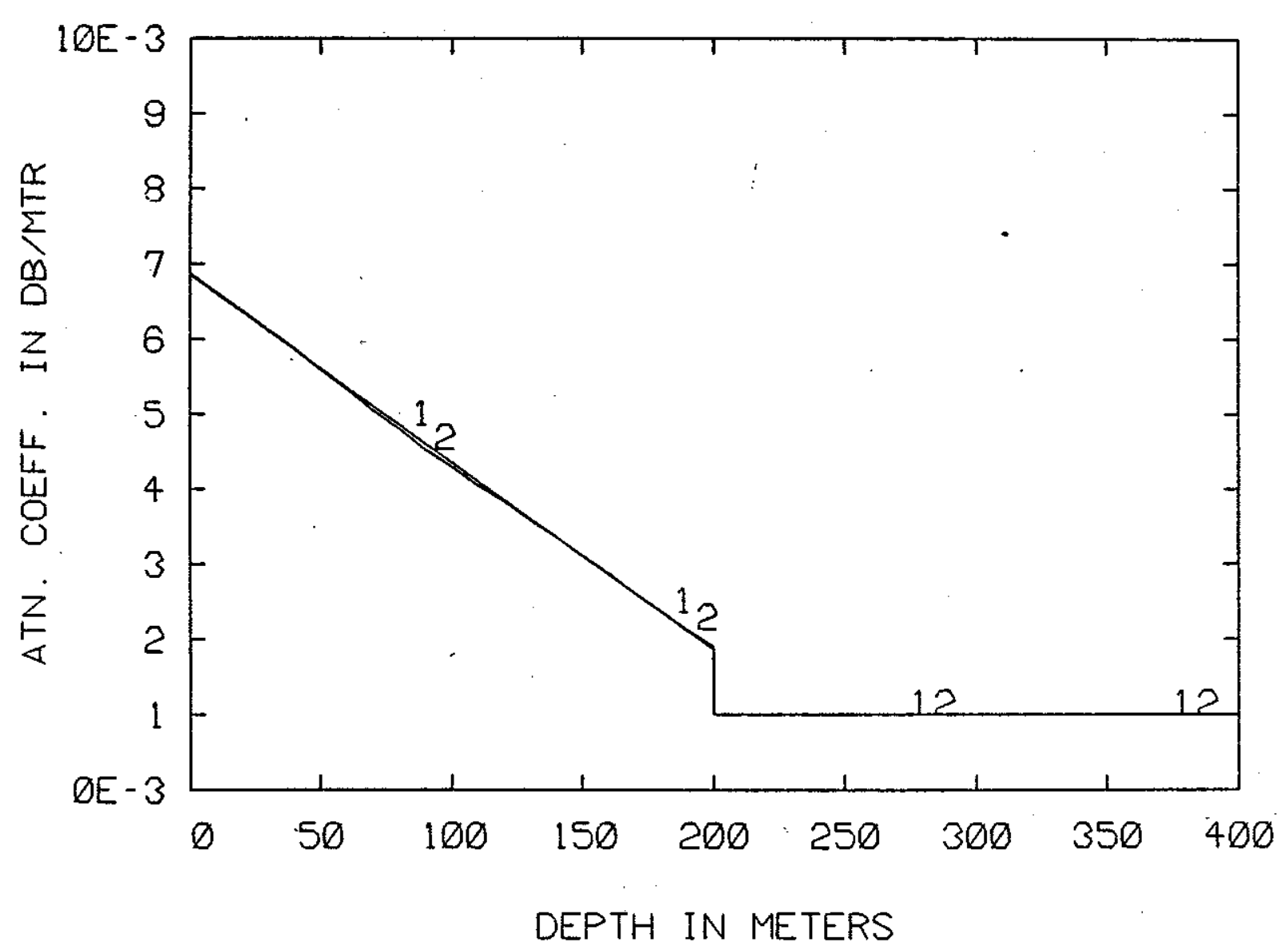

Figure 7-16: Reconstruction of profile in figure 7-14(b) 1- True profile, 2-Reconstructed profile 


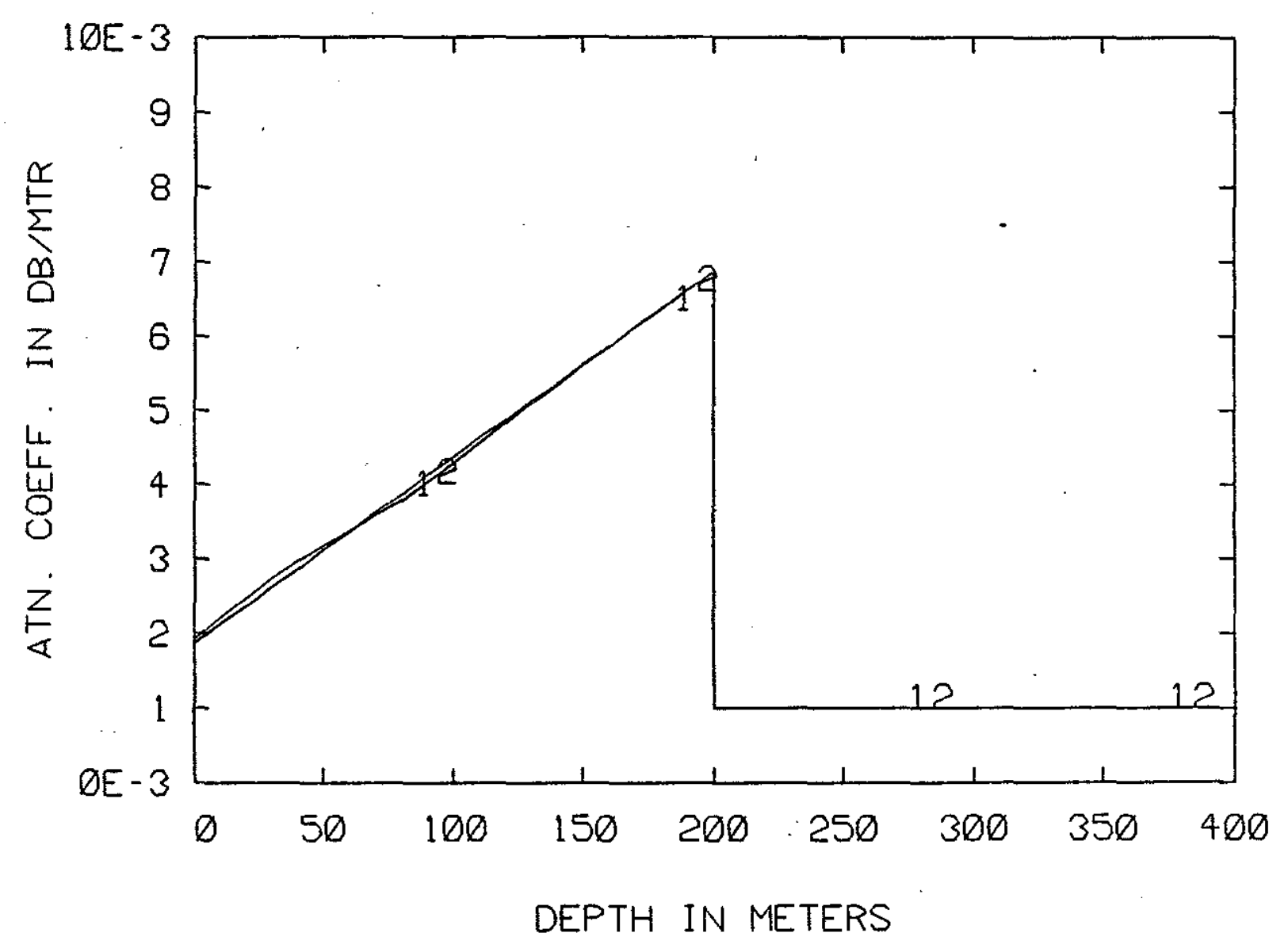

Figure 7-17: Reconstruction of profile in figure 7-14(c)

1- True profile, 2- Reconstructed profile 


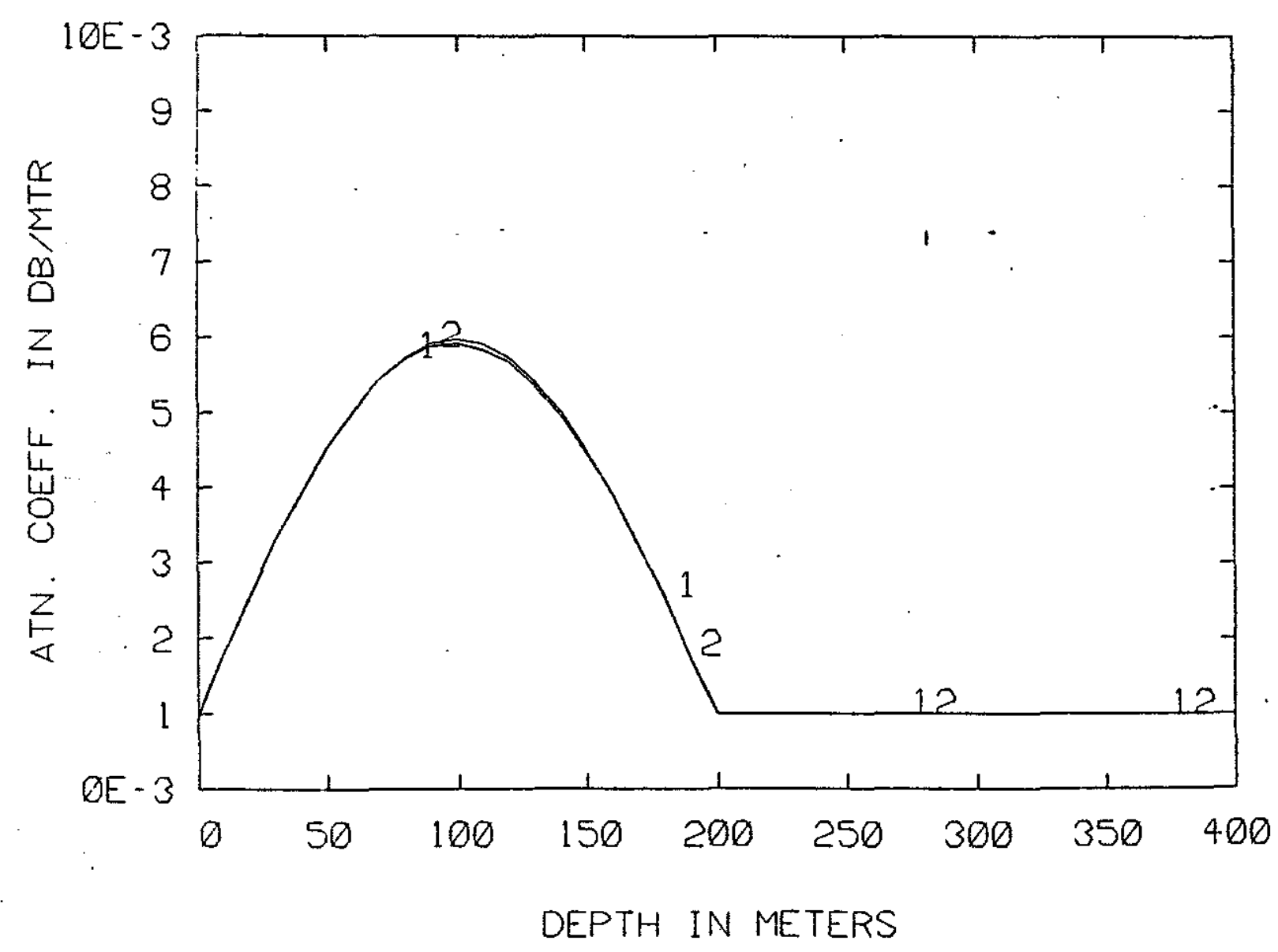

Figure 7-18: Reconstruction of profile in figure 7-14(d)

1- True profile, 2- Reconstructed profile 
$-168-$

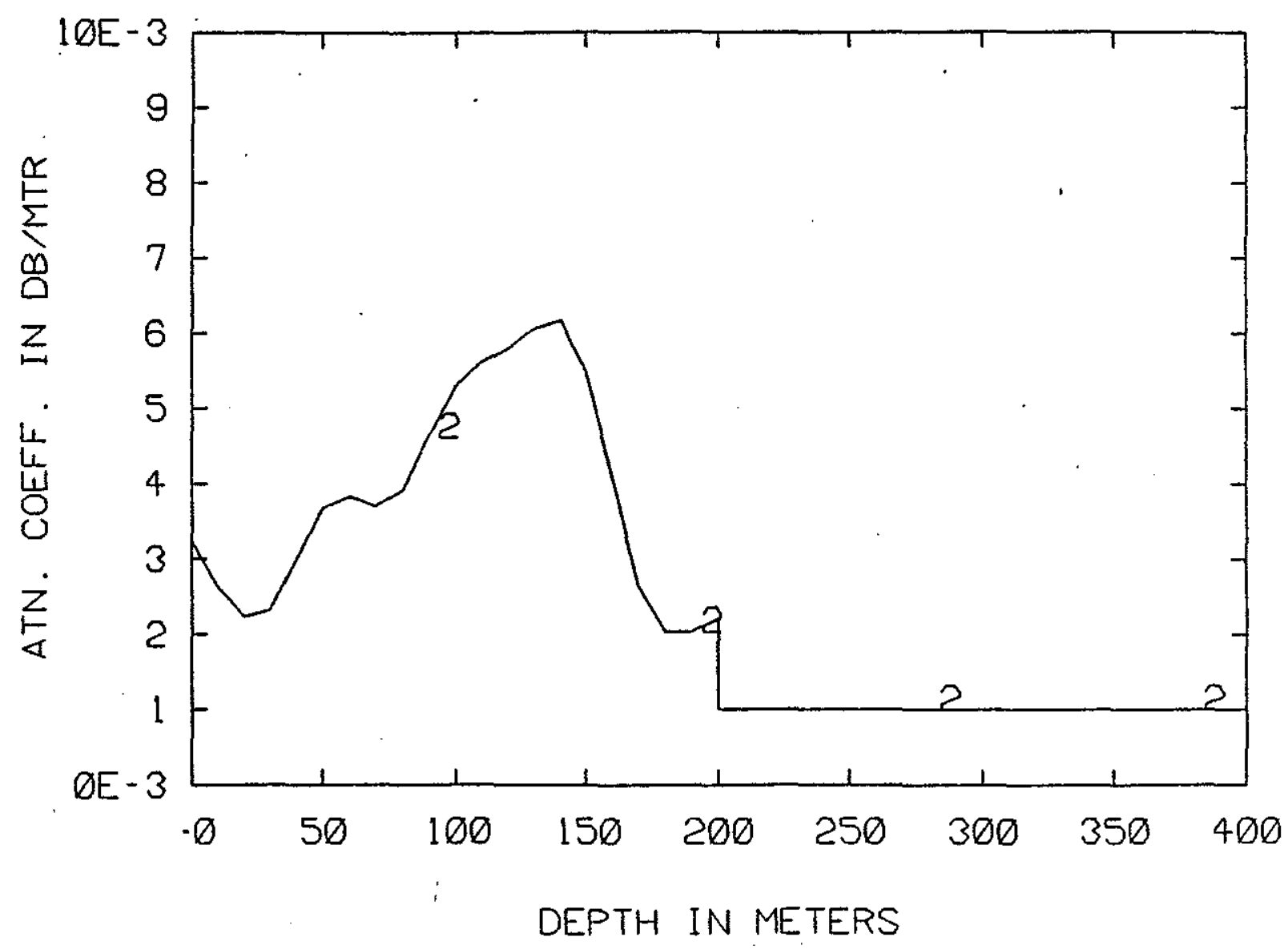

Figure 7-19: Reconstruction of profile in figure 7-14(e)

2- Reconstructed profile 


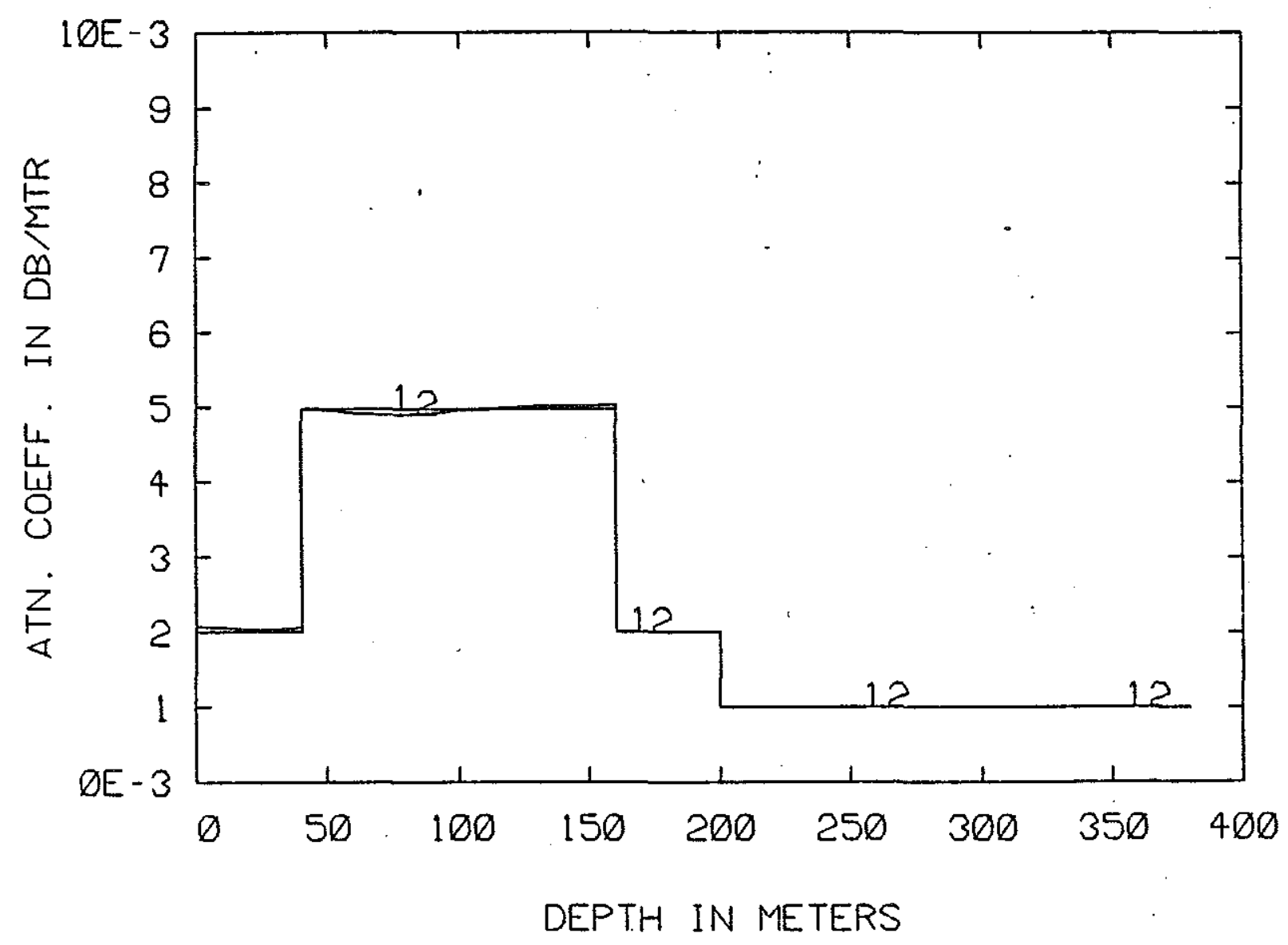

Figure 7-20: Reconstruction of profile in figure 7-14(e) 1- True profile, 2- Reconstructed profile 


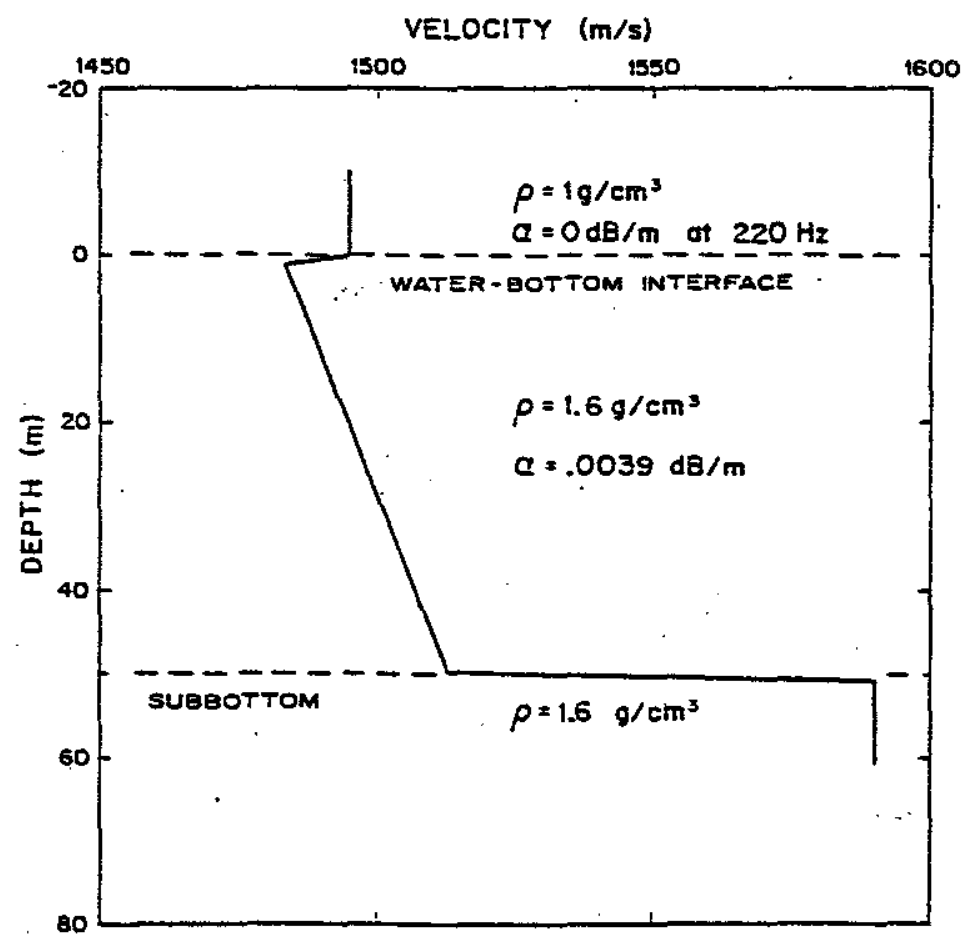

Figure 7-21: Realistic ocean model 


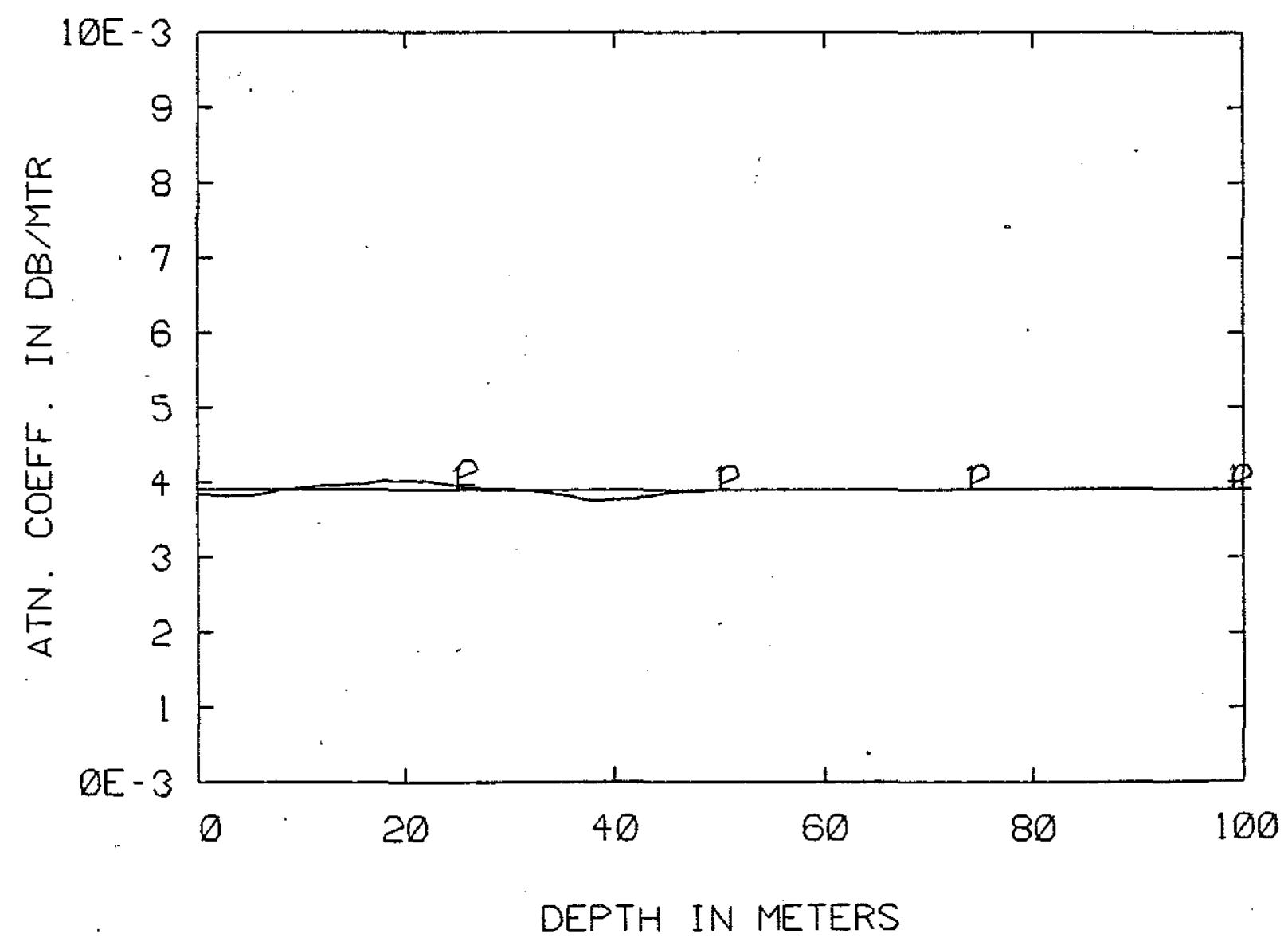

Figure 7-22: Reconstruction of profile for realistic ocean model 1- True profile, 2- Reconstructed profile 
4. The input data is obtained at 20 discrete points in the pre-critical range. For the sake of convenience the input data is computed at equal increments of vertical wavenumber. An operating frequency of $25 \mathrm{~Hz}$ was chosen. This was done only to limit the size of the matrices since the sampling interval is dependent on the wave length in the water column as explained in an earlier section. The sound speed structure in the sediment was obtained using the regression formula given by Hamilton[8]. The density in the sediment layer has been assumed to be constant. The values for these parameters are given in Table 7-I. The Lagrange multiplier was suitably chosen and the iteration continued till residual became less than a predetermined value based on the numerical errors that may creep into the computation of the reflection coefficient.

For the iteration scheme to converge the nonlinearity should not be strong. Stating differently, the guess model must be close to the real model. In Chapter 5 we saw that the attenuation coefficient is orders of magnitude smaller than the real part of the wavenumber and this was the foundation on which we based the perturbation method. At $25 \mathrm{~Hz}$ the real part of the wavenumber is approximately $0.1 \mathrm{~m}^{-1}$. Therefore the initial guess value for the attenuation is taken as zero. For values of attenuation assumed the convergence occured in about six iterations.

The results of the inversion are given in Figures 7-15 to 7-19. Except in the case of Figure 7-19 which is the recontructed profile for the discontinuous model at Figure 7-14 the reconstruction is good with the residuals less than $10^{-7}$. The poor reconstruction in the case of Figure 7-19 is due to the fact that the algorithm assumes that the function we are looking for is smooth whereas in reality it is discontinuous. The points where such discontinuities are likely to be, are seen in the Figure 7-19. Based on this evidence we make the assumption that the discontinuities do occur at these depths and look for smooth solutions in the three 
separate regions of depth. Then the result in Figure 7-20 is obtained.

We now give an example of inversion using one of the realistic models developed for Icelandic Basin[9]. The operating frequency in this instance is $220 \mathrm{~Hz}$, the frequency at which the experiment was performed. The model used and the reconstructed profile are given in Figures 7-21 and 7-22.

\subsection{Born and Rytov approximations}

The question about the applicability of the Rytov approximation for back scattering problems is still unresolved. While it can be proved [10] that the Rytov approximation is valid over longer ranges than the Born approximation in regions where only one field exists, no such general result exists for regions where there is more than one wave. According to Keller[10], where more than one wave exists, the Rytov approximation is to be applied to each wave seperately and not to the total field if its validity over longer ranges than the Born method is to hold. On the other hand the Born approximation can be applied to the total field. This accounts for the more common use of the Born approximation for backscattering problems. However, the investigations on the relative merits of the two methods for ultrasonic diffraction tomography have shown that the Rytov method gives better results than the Born approximation even when the Rytov is applied to the total field[11,12]. In the light of these comments, we now carry out the inversion without the iterative scheme and compare the results obtained by the two methods. The results are given in Figures 7-23 to 7-26. We note that the Rytov method is much better than the Born approximation at shallower depths and only slightly worse than the Born approximation at deeper depths. This experiment, therefore, seems to confirm the observation of other investigators that the Rytov method is 


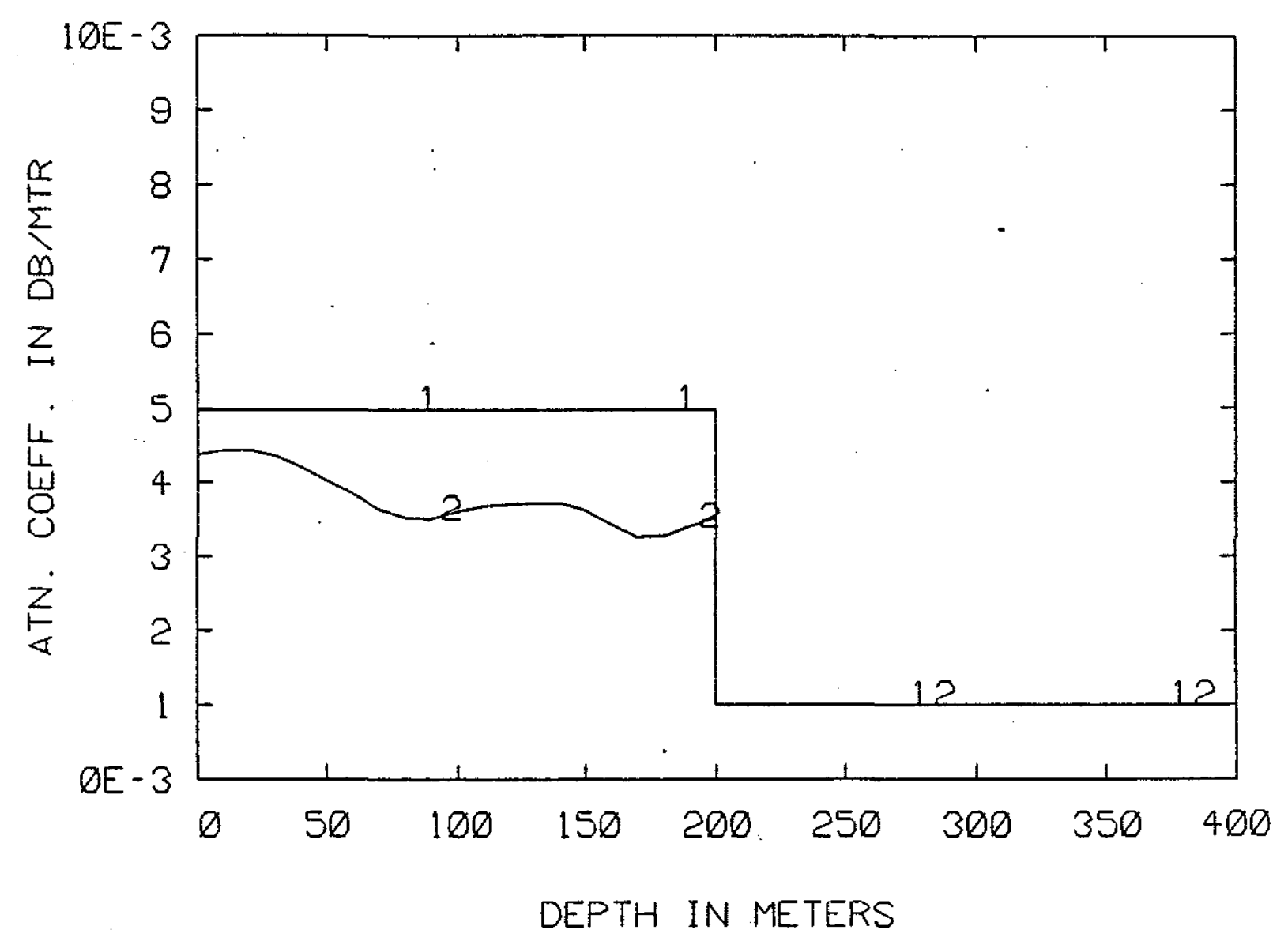

Figure 7-23: Reconstruction of profile in figure 7-14(a) - Born approximation

1- True profile, 2- Reconstructed profile 
$-175-$

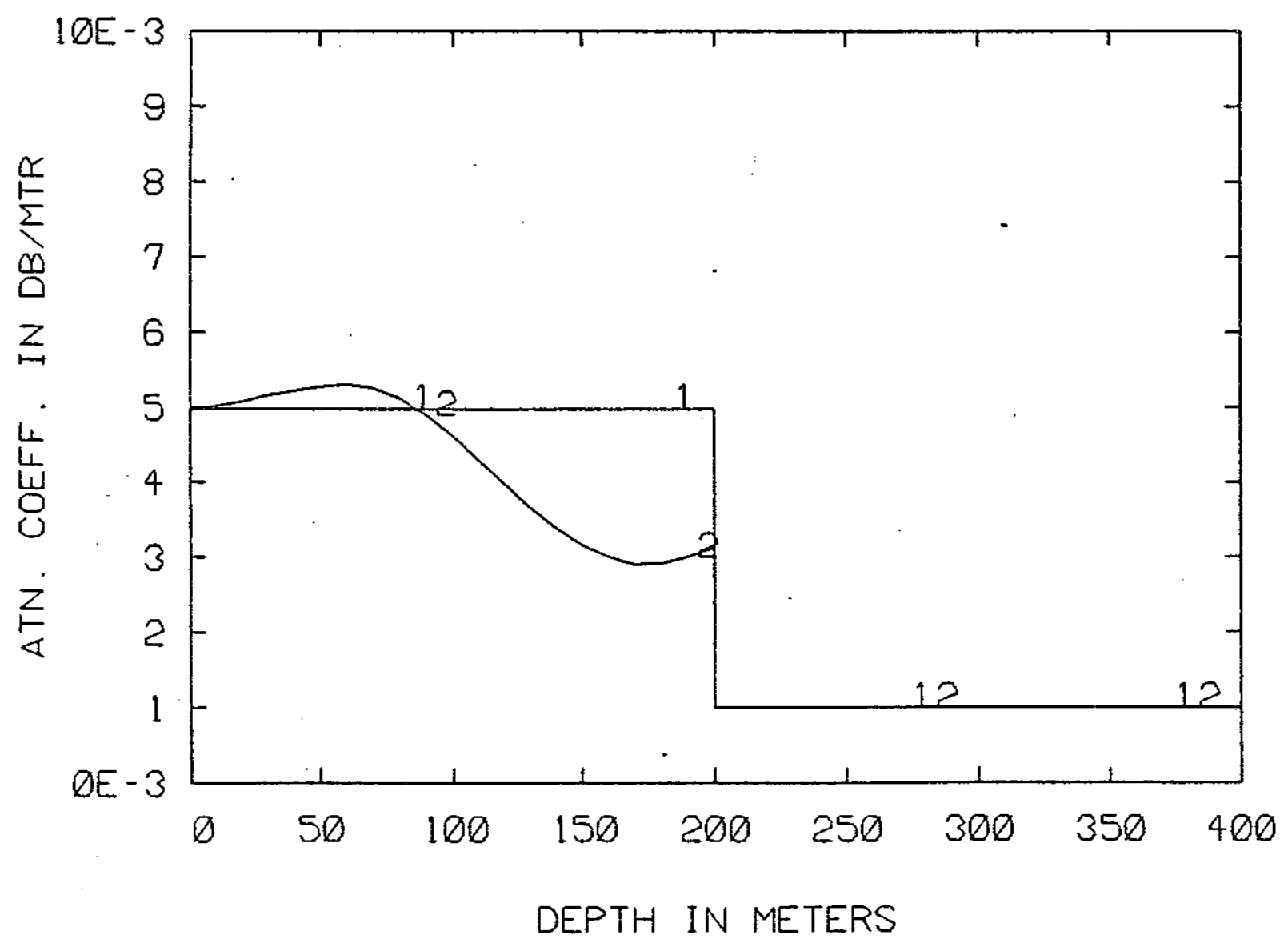

Figure 7-24: (Reconstruction of profile in figure 7-14(a) - Rytov approximation

1- True profile, 2- Reconstructed profile 


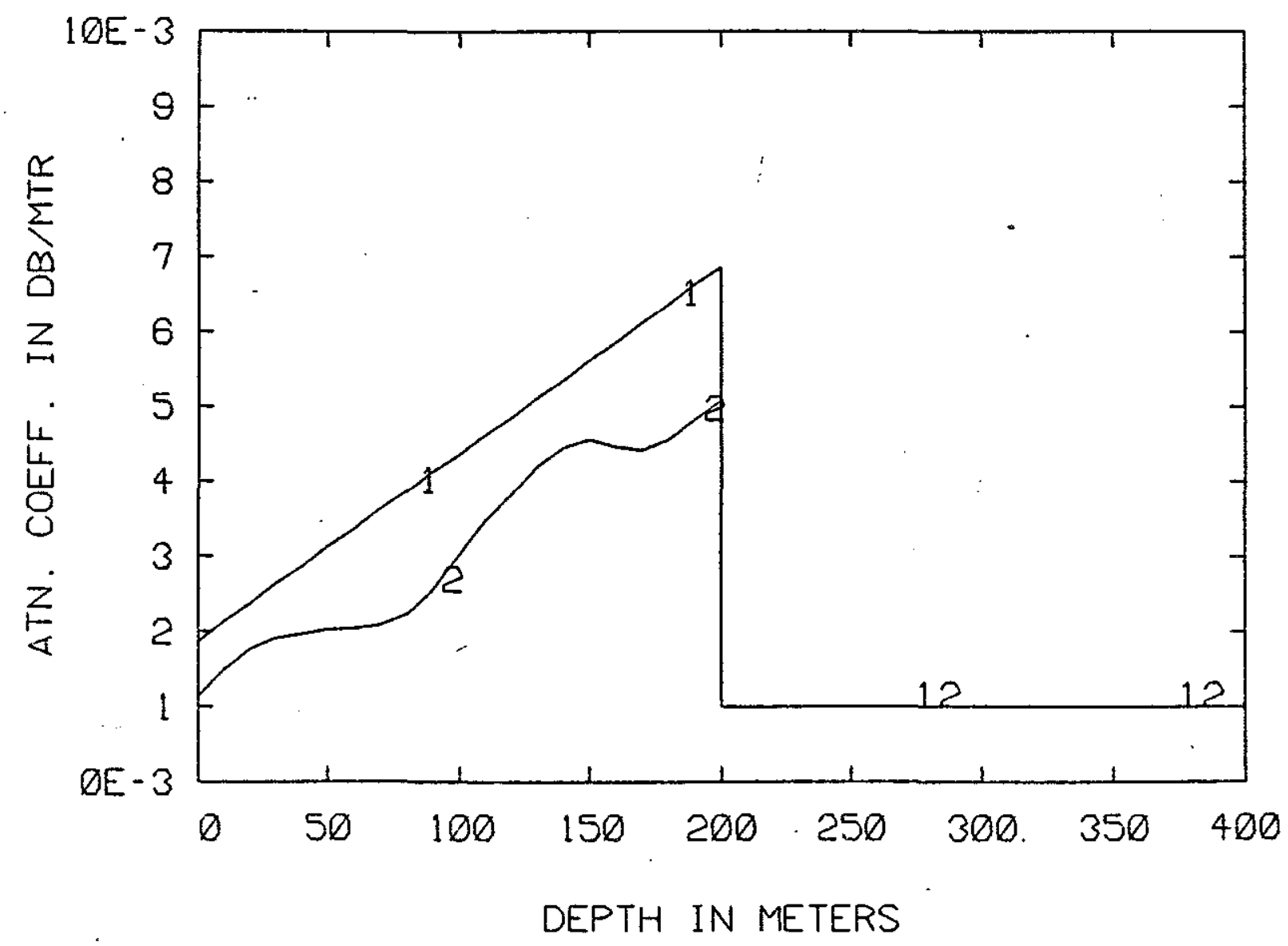

Figure 7-25: Reconstruction of profile in figure 7-14(b) - Born approximation

1- True profile, 2- Reconstructed profile 


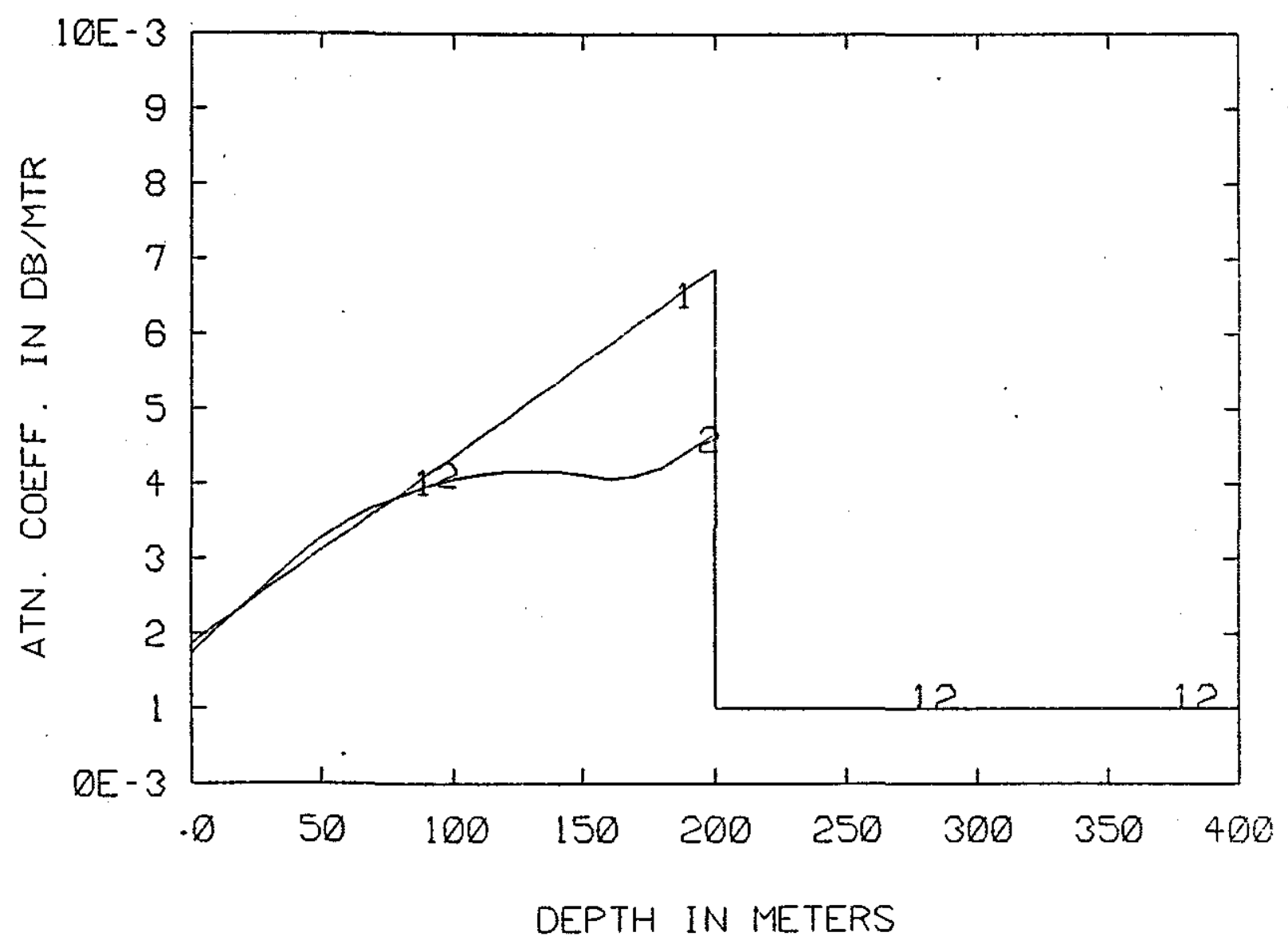

Figure 7-26: Reconstruction of profile in figure 7-14(b)- Rytov approximation

1- True profile, 2- Reconstructed profile 
$-178$

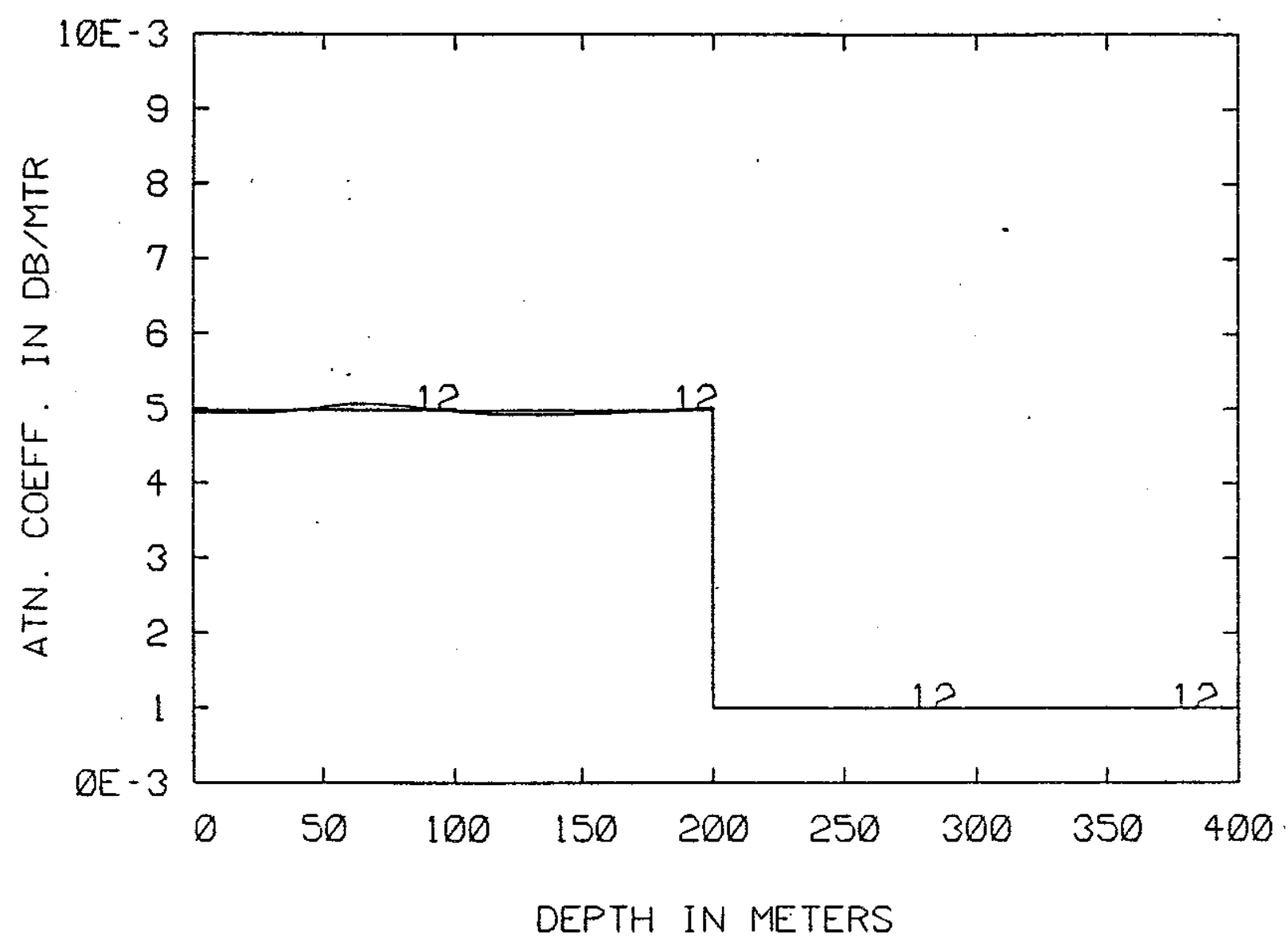

Figure 7-27: Reconstruction of profile in figure 7-14(a) - Rytov approximation

1- True profile, 2-Reconstructed profile 
better than the Born method even in situations where more than one field exists. The analytical foundations for this result needs to be established to determine the limitations in the use of Rytov's method. However, since an iterative process is found necessary to solve the non-linear problem, both methods needed approximately the same number of iterations to converge Therefore, neither of the two methods can be considered to have a clear advantage over the other. The iterated result using Rytov's method for the profile in Figure 7-14 (a) is shown in Figure 7-27

\subsection{Resolution}

Resolution is a measure of our ability to resolve fine structure in the function being reconstructed given that we have only a finite set of data points. A method of determining the resolvability is given by Backus and Gilbert[1]. Here the procedure described by them is extended to cover situations where the data, the kernel and the function to be obtained can all be complex quantities. The situation when the unknown function is a complex quantity arises when we invert to obtain the real and imaginary part of the wavenumber.

We start by considering the linear problem. The data is related to the unknown through a linear equation of the form given below. All the quantities are complex.

$$
d_{n}=\int_{0}^{h} \gamma(z) G_{n}(z) d z
$$

Multiplying by a weight $a_{n}\left(z_{0}\right)$ and summing over all n's we obtain, 


$$
\sum_{n} d_{n} a_{n}\left(z_{0}\right)=\int_{0}^{h} \gamma(z)\left\{\sum_{n} a_{n}\left(z_{0}\right) G_{n}(z)\right\} d z
$$

or,

$$
d\left(z_{0}\right)=\int_{0}^{h}\left(\gamma(z) A\left(z, z_{0}\right) d z\right.
$$

Let $d\left(z_{0}\right), A\left(z, z_{0}\right)$ and $\gamma(z)$ be all complex. Equating the real and imaginary parts, we obtain

$$
d_{r}=\int_{0}^{h}\left\{\gamma_{r}(z) A_{r}\left(z, z_{0}\right)-\gamma_{i}(z) A_{i}\left(z, z_{0}\right)\right\} d z
$$

and

$$
d_{i}=\int_{0}^{h}\left\{\gamma_{r}(z) A_{i}\left(z, z_{0}\right)+\gamma_{i}(z) A_{r}\left(z, z_{0}\right)\right\} d z
$$

The subscript $r$ and $i$ represent the real and imaginary parts. If the coefficients $a_{n}\left(z_{0}\right), n=1, \cdots, N$ can be found such that $A_{i}\left(z, z_{0}\right)$ is equal to $\delta\left(z-z_{0}\right)$ and $A_{i}\left(z, z_{0}\right)$ is zero, then we will have

$$
d_{r}\left(z_{0}\right)=\int_{0}^{h} \gamma_{r}(z) \delta\left(z-z_{0}\right) d z
$$

and

$$
d_{i}\left(z_{0}\right)=\int_{0}^{h} \gamma_{i}(z) \delta\left(z-z_{0}\right) d z
$$


$\gamma_{r}\left(z_{0}\right)$ and $\gamma_{i}\left(z_{0}\right)$ will then be known exactly. Similarly for each point we can find a set of coeficents which will make $A_{r}\left(z, z_{0}\right)$ look like a delta function and $A_{i}\left(z, z_{0}\right)$ equal to zero. However, this cannot be done. We, therfore, seek to minimise,

$$
\int_{0}^{h}\left\{A_{r}\left(z-z_{0}\right)-\delta\left(z-z_{0}\right)\right\}^{2} d z+\int_{0}^{h} A_{i}^{2}\left(z-z_{0}\right) d z
$$

subject to $A_{r}\left(z, z_{0}\right)$ being unimodular i.e.

$$
\int_{0}^{h} A_{r}\left(z, z_{0}\right) d z=1
$$

Other measures of 'deltaness' have been proposed in literature.[1]

From equation (7.44) the expressions for $A_{r}\left(z, z_{0}\right)$ and $A_{i}\left(z, z_{0}\right)$ are obtained. Substituting for $A_{r}\left(z, z_{0}\right)$ and $A_{i}\left(z, z_{0}\right)$ in the equation (7.46), we minimise,

$$
\begin{aligned}
\int_{0}^{h}\left[\sum_{n} a_{n}\left(z_{0}\right) G_{n r}(z)-\delta\left(z-z_{0}\right)\right]^{2} d z & +\int_{0}^{h}\left[\sum_{n} a_{n}\left(z_{0}\right) G_{n i}(z)\right]^{2} d z \\
& +\lambda \int_{0}^{h}\left[\sum a _ { n } \left(z_{0} G_{n r}(z) d z\right.\right.
\end{aligned}
$$

where $\lambda$ is the lagrange multiplier. The derivative of this expression with respect to each of the coefficient $a_{n}$ is set to zero. A set of $n$ linear equations is obtained which are then solved to obtain the coefficients. After some algebra the following equation is obtained.

$$
2\left(\Psi_{r}+\Psi_{i}\right) \mathbf{a}+\lambda \mathbf{u}=2 \mathrm{G}_{r}\left(z_{0}\right)
$$

where 
$\left(\psi_{r}\right)_{n m}=\int_{0}^{h} G_{n r}(z) G_{m r}(z) d z$

$\left(\psi_{i}\right)_{n m}=\int_{0}^{h} G_{n i}(z) G_{m i}(z) d z$

$\mathrm{a}=\left\{a_{1}\left(z_{0}\right), \cdots, a_{n}\left(z_{0}\right)\right\}^{T}$

$\mathrm{G}_{r}\left(z_{0}\right)=\left\{G_{1 r}\left(z_{0}\right), \cdots, G_{n r}\left(z_{0}\right)\right\}^{T}$

$u_{n}=\int_{0}^{h} G_{n r}(z) d z$

$\mathrm{u}=\left\{u_{1}, \cdots, u_{n}\right\}^{T}$

Let,

$$
\Psi=\Psi_{r}+\Psi_{i}
$$

Then,

$$
2 \boldsymbol{W a}+\lambda \mathbf{u}=2 \mathrm{G}_{\mathbf{r}}\left(z_{0}\right)
$$

Or,

$$
\mathrm{a}=\boldsymbol{\Psi}^{-1}\left[\mathrm{G}_{r}\left(z_{0}\right)-\lambda \mathbf{u} / 2\right]
$$

We now use the constraint relation given below.

$$
\mathbf{a}^{T} \mathbf{u}=1
$$

Sustituting for a, we obtain 


$$
\mathbf{u}^{T} \Psi\left[\mathrm{G}_{r}\left(z_{0}\right)-\lambda \mathbf{u} / 2\right]=1
$$

or,

$$
\mathbf{a}=\Psi^{-1}\left[\mathrm{G}_{r}\left(z_{0}\right)-\left\{\frac{\mathbf{u}^{T} \Psi^{-1} \mathbf{G}_{r}\left(z_{0}\right)-1}{\mathbf{u}^{T} \Psi^{-1} \mathbf{u}}\right\} \mathbf{u}\right]
$$

Having found a $\mathrm{a}_{\mathrm{n}}$, we can construct $A_{r}\left(z, z_{0}\right)$ and $A_{i}\left(z, z_{0}\right) . A_{r}\left(z, z_{0}\right)$ is a measure of the resolution. Further $A_{i}\left(z, z_{0}\right)$ is a measure of the effect of the imaginary part on the recontruction of the real part and vice-versa and is therefore a measure of contamination.

The above method is applicable when the equation is linear. In the non-linear problem, the resolution kernels are obtained by the method suggested by Parker[13]. The linearisation assumption is based on the last term in equation (7.2) being negligible. If this is not small, the solution we obtain will not satisfy data. By iterating we obtain an acceptable solution and when such a solution is obtained we can say that the term $\mathrm{O}\|\gamma\|^{2}$ is negligible. The Frechet kernels obtained with this solution is the used to generate the resolution kernels.

\subsubsection{Measure of resolution}

Resolution length defined as given below is a measure of resolution[14].

$$
R L\left(z_{0}\right)=\frac{1}{h} \int_{0}^{h A^{2}\left(z, z_{0}\right)} d z
$$

While resolution length at each point $\mathrm{z}$ gives a measure of local resolution one can also obtain a measure for global resolution. 

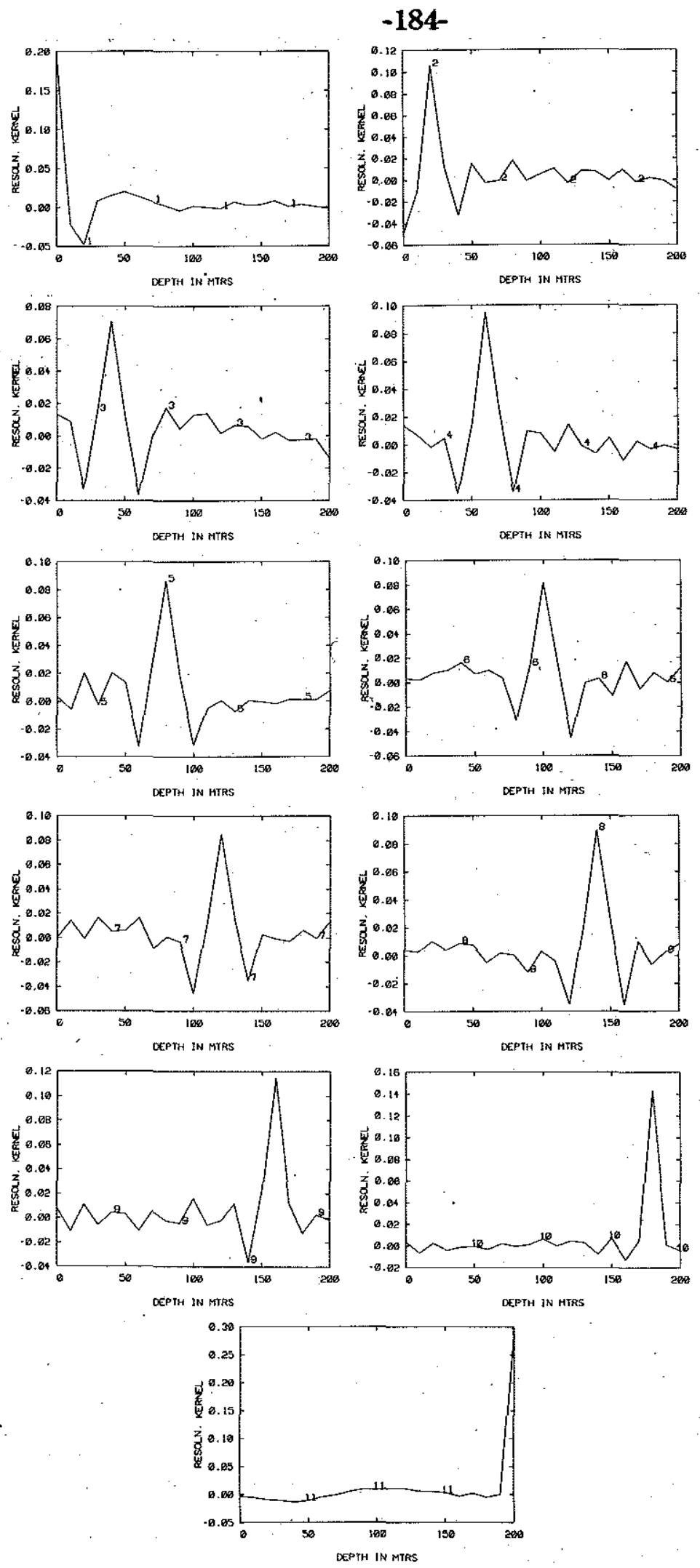

Figure 7-28: Resolving kernel for profile 7-14(a) 


\section{$-185-$}
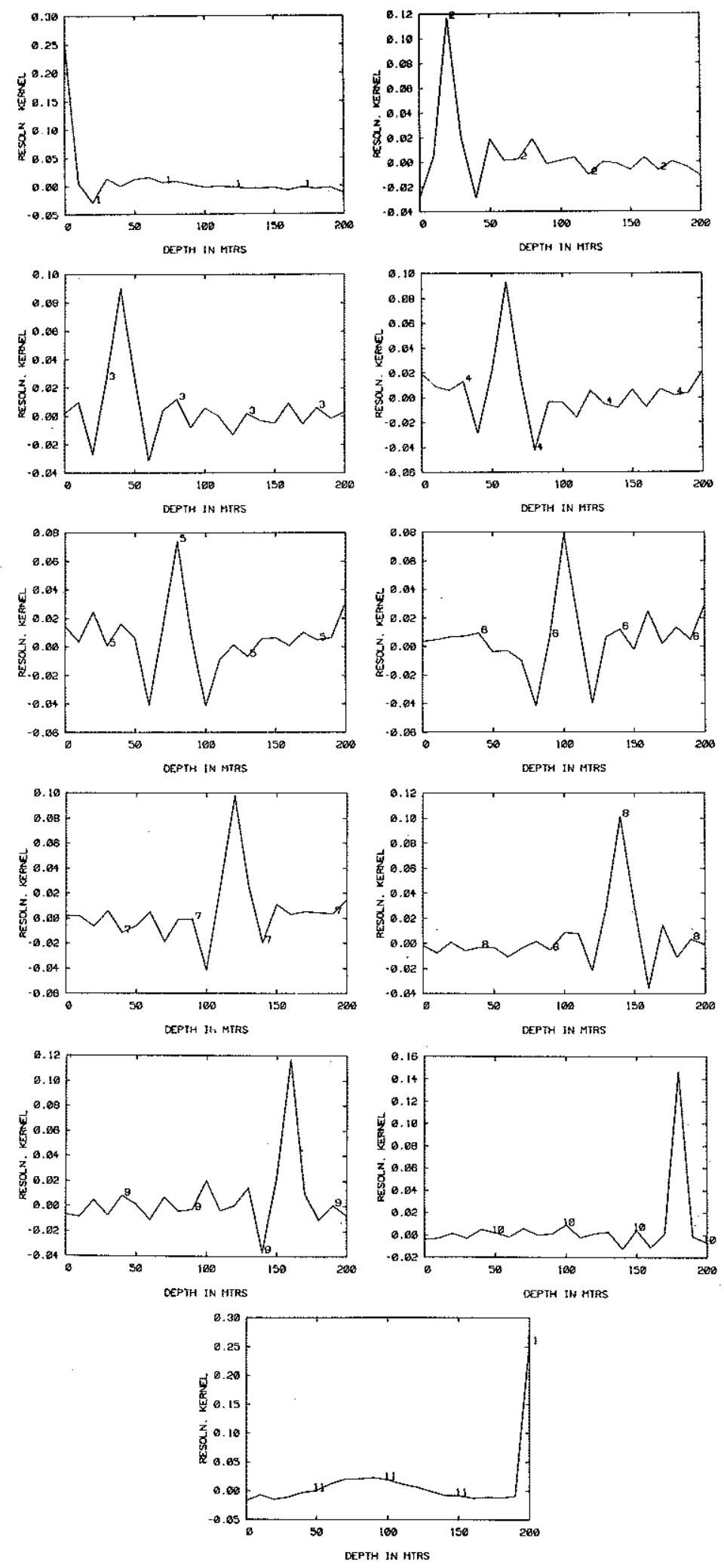

Figure 7-29: Resolving kernel for profile 7-14(b) 
$-186-$
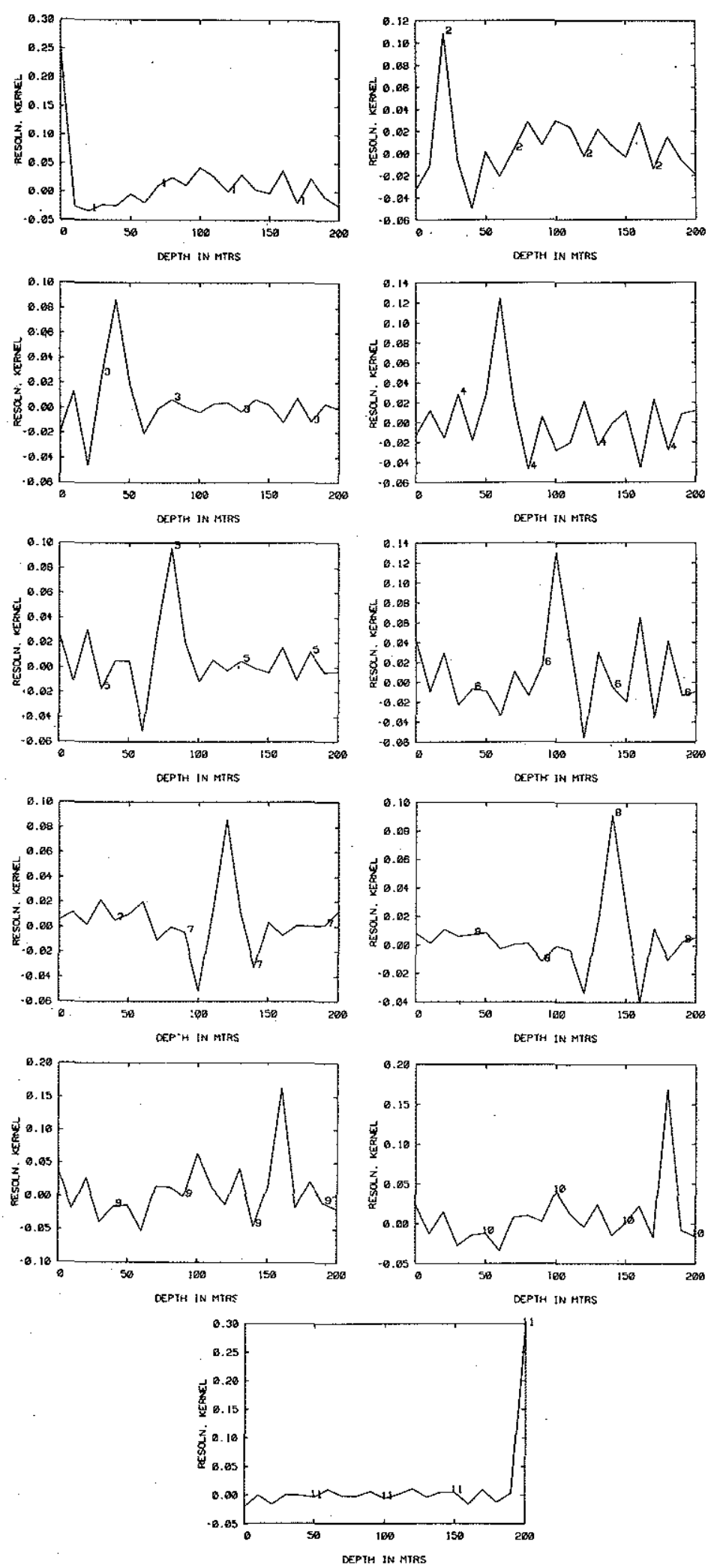

Figure 7-30: Resolving kernel for profile 7-14 (c) 
$-187-$
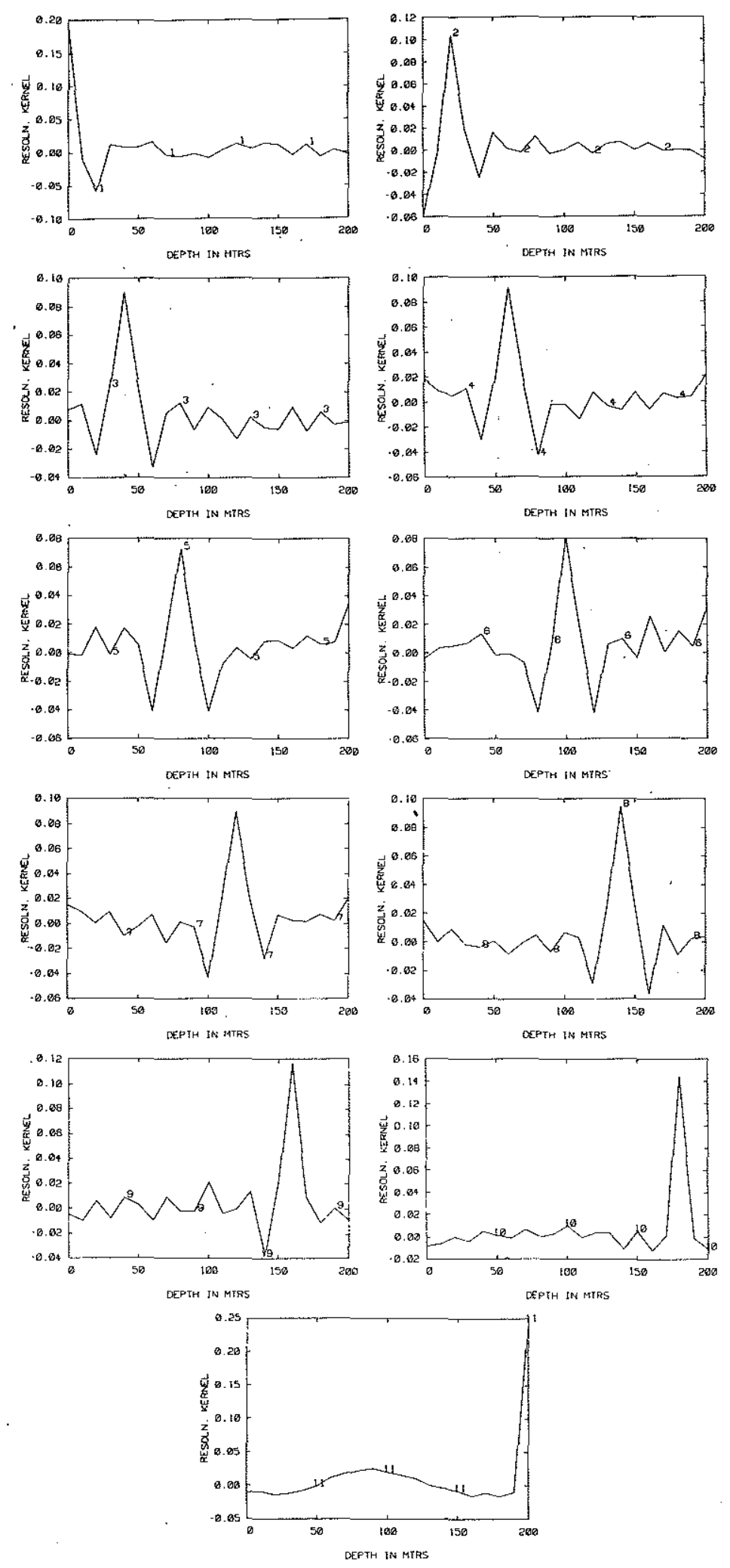

Figure 7-31: Resolving kernel for profile 7-14 (d) 
$-188$

\begin{tabular}{|c|c|c|c|c|}
\hline Depth(m) & Profile fig. 14(a) & Profile fig. $14(b)$ & Profile fig. $14(\mathrm{c})$ & Profile fig. $14(d)$ \\
\hline 0 & $2.15 \times 10^{-2}$ & $1.82 \times 10^{-2}$ & $2.48 \times 10^{-2}$ & $2.91 \times 10^{-2}$ \\
\hline 20 & $5.48 \times 10^{-2}$ & $5.02 \times 10^{2}$ & $7.31 \times 10^{-2}$ & $5.38 \times 10^{-2}$ \\
\hline 40 & $8.51 \times 10^{-2}$ & $7.22 \times 10^{-2}$ & $7.54 \times 10^{-2}$ & $7.17 \times 10^{-2}$ \\
\hline 60 & $7.15 \times 10^{-2}$ & $7.59 \times 10^{-2}$ & $8.42 \times 10^{-2}$ & $7.58 \times 10^{-2}$ \\
\hline 80 & $7.83 \times 10^{-2}$ & $9.79 \times 10^{-2}$ & $8.87 \times 10^{-2}$ & $9.81 \times 10^{-2}$ \\
\hline 100 & $8.45 \times 10^{-2}$ & $9.27 \times 10^{-2}$ & $9.91 \times 10^{-2}$ & $9.24 \times 10^{-2}$ \\
\hline 120 & $8.28 \times 10^{-2}$ & $7.25 \times 10^{-2}$ & $8.72 \times 10^{-2}$ & $7.72 \times 10^{2}$ \\
\hline 140 & $7.50 \times 10^{-2}$ & $7.04 \times 10^{-2}$ & $7.54 \times 10^{-2}$ & $7.19 \times 10^{-2}$ \\
\hline 160 & $6.04 \times 10^{-2}$ & $6.04 \times 10^{-2}$ & $7.91 \times 10^{-2}$ & $6.11 \times 10^{-2}$ \\
\hline 180 & $4.22 \times 10^{-2}$ & $4.22 \times 10^{-2}$ & $5.27 \times 10^{-2}$ & $4.25 \times 10^{-2}$ \\
\hline 200 & $1.76 \times 10^{-2}$ & $1.94 \times 10^{-2}$ & $1.76 \times 10^{-2}$ & $2.00 \times 10^{-2}$ \\
\hline GRL & $6.73 \times 10^{-2}$ & $6.86 \times 10^{-2}$ & $7.65 \times 10^{-2}$ & $6.86 \times 10^{-2}$ \\
\hline
\end{tabular}

Table 7-II: Resolution lengths for the different examples of reconstruction 


$$
G R L=\frac{\mathbb{1}}{h} \int_{0}^{h} R L\left(z_{0}\right) d z_{0}
$$

The global resolution measure can be used to determine the effect of various parameters in inversion.

For the various examples of the reconstruction of the attenuation coefficient profile the resolution kernels have been constructed adopting a procedure similar to the one discussed above except that the function $\gamma(z)$ is real. There will, therefore, no contamination from the imaginary part i.e we need to determine only $A_{r}\left(z, z_{0}\right)$. These results are given in Figures 7-28 to 7-31. The resolution lengths for the cases are given in Table 7-II.

The average resolution length is approximately .07 which corresponds to 14 $\mathrm{m}$. The layer thickness has been taken as $10 \mathrm{~m}$ and within each layer the attenuation is taken as constant. A resolution length of $14 \mathrm{~m}$ can be considered as adequate in resolving the features of the model.

We will now see how the aperture size and the number of data points affect resolution. We will use the global resolution measure to study this effect.

\subsubsection{Variation of aperture size}

Keeping the number of data points same, the aperture size is varied and for each case the resolution length and the global resolution length are caiculated. The resolution length and global resolution measure are given in Table 7-III . As the angular aperture increases the resolution improves. With small angular aperiure, the separation between data points is small. In the ray picture the rays probing the medium will be close to each other leading to near dependency of the rows of the 
Angular aperture

\begin{tabular}{|c|c|c|c|}
\hline Depth & $\left(.054<\mathrm{K}_{20}<.067\right)$ & $\left(.054<\mathrm{K}_{20}<.079\right)$ & $\left(.054<\mathrm{K}_{20}<.088\right)$ \\
\hline 0 & $6.6 \times 10^{-2}$ & $1.84 \times 10^{-2}$ & $2.15 \times 10^{-2}$ \\
\hline $20 \mathrm{~m}$ & 0.103 & $6.91 \times 10^{-2}$ & $5.48 \times 10^{-2}$ \\
\hline $40 \mathrm{~m}$ & 0.110 & $8.95 \times 10^{-2}$ & $8.51 \times 10^{-2}$ \\
\hline $60 \mathrm{~m}$ & 0.109 & $8.73 \times 10^{-2}$ & $7.15 \times 10^{-2}$ \\
\hline $80 \mathrm{~m}$ & 0.111 & $9.28 \times 10^{-2}$ & $7.83 \times 10^{-2}$ \\
\hline $100 \mathrm{~m}$ & 0.121 & 0.112 & $8.45 \times 10^{-2}$ \\
\hline $120 \mathrm{~m}$ & 0.128 & $9.24 \times 10^{-2}$ & $8.28 \times 10^{-2}$ \\
\hline $140 \mathrm{~m}$ & 0.104 & $8.75 \times 10^{-2}$ & $7.50 \times 10^{-2}$ \\
\hline $160 \mathrm{~m}$ & 0.101 & $8.03 \times 10^{-2}$ & $6.04 \times 10^{-2}$ \\
\hline $180 \mathrm{~m}$ & $7.84 \times 10^{-2}$ & $4.90 \times 10^{-2}$ & $4.22 \times 10^{-2}$ \\
\hline $200 \mathrm{~m}$ & $1.79 \times 10^{-2}$ & $1.76 \times 10^{-2}$ & $1.76 \times 10^{-2}$ \\
\hline $\mathrm{GRL}$ & $9.98 \times 10^{-2}$ & $7.89 \times 10^{-2}$ & $6.73 \times 10^{-2}$ \\
\hline & & & \\
\hline & & & \\
\hline
\end{tabular}

Table 7-III: Resolution length and aperture size 
No of Data pts.

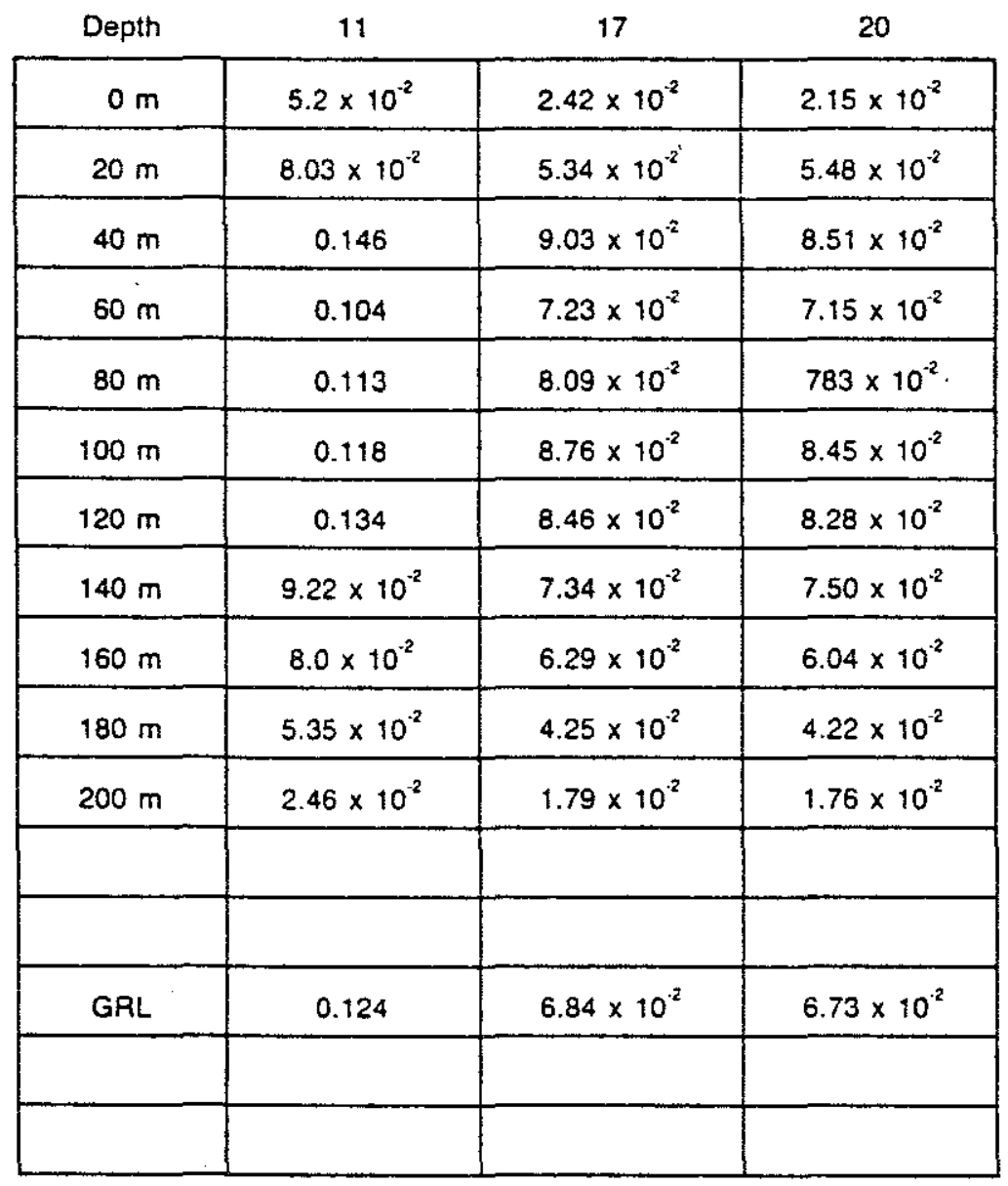

Table 7-IV: Resolution length and number of data points 
$-192-$

matrix we are trying to invert. This illconditioning has to be overcome by discarding eigenvectors corresponding to low eigenvalues and therefore leads to poor resolution. However as the aperture size increases the near dependency of the rows gets reduced and the resolution improves.

\subsubsection{Variation of the number of data points}

For this experiment, the resolution length and global resolution length were obtained for number of data equal to 11,17 and 20. These are given in Table 7-IV. As the number of data points increase the resolution improves. This is what one would anticipate as the small number of points makes the system underdetermined with a number of eigenvalues close to zero. A smooth solution is obtained by utilising a relatively small number of eigenvectors which degrades the resolution.

\subsection{Errors}

So far we have assumed that the sound speed and density for the sediment are known exactly. In the sequence of processing of the measured data as shown in Figure (3-7) of Chapter 3, the sound speed and density values are obtained using direct inverse algorithms. These algorithms assume that the sediment is lossless and since this assumption is not true, it will give rise to errors in the reconstructed values of the sound speed and density. To show this we do the following synthetic experiment. Merab[15] has developed an inverse scheme based on the GelfandLevitan method for obtaining the sound speed and density profiles for the sediment. We generated reflection coefficients synthetically using a lossy model and this was used in the inversion scheme to obtain the value of sound speed. The density in the sediment was assumed known. The procedure was repeated with a different set of 

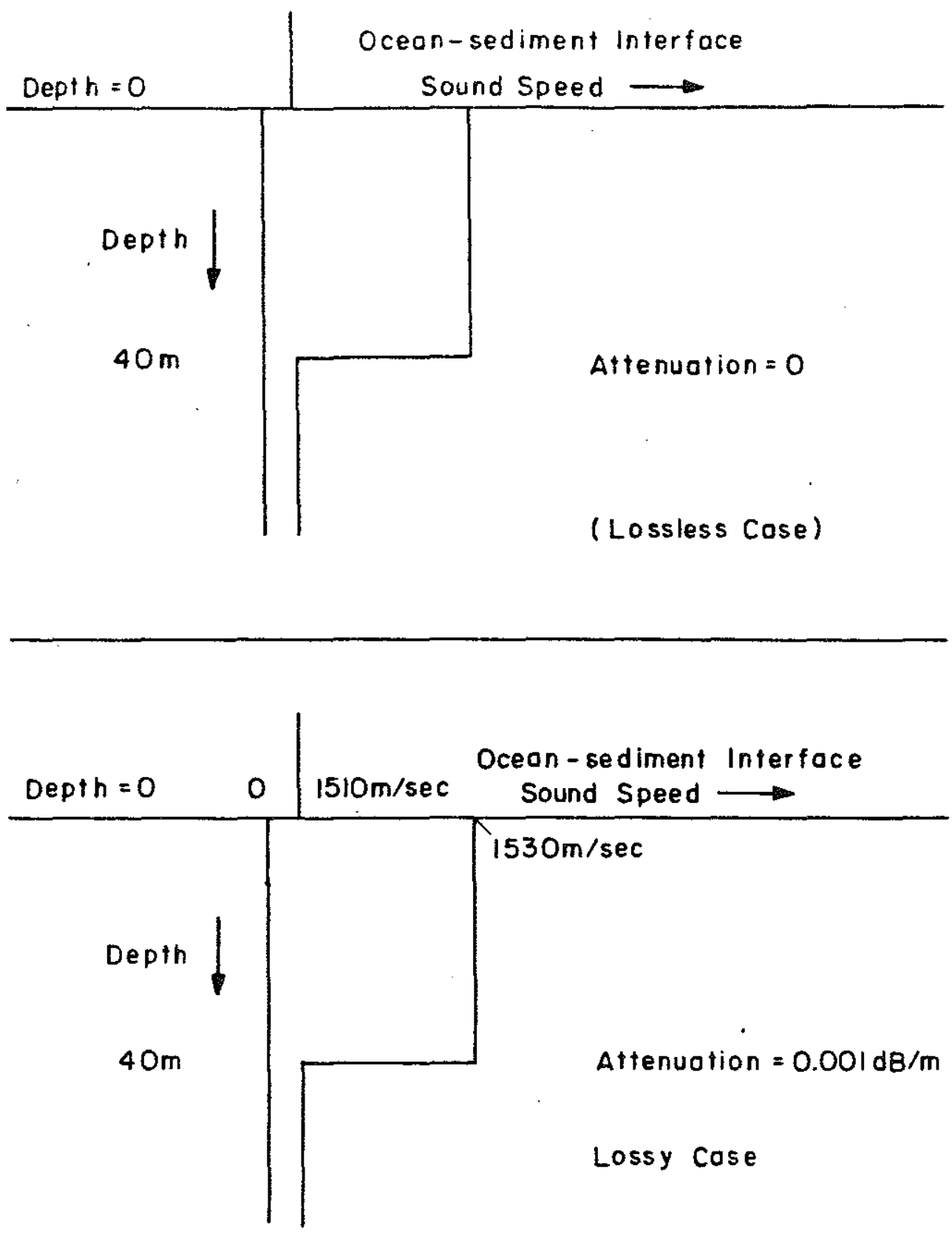

Figure 7-32: Ocean botiom model 
$-194-$

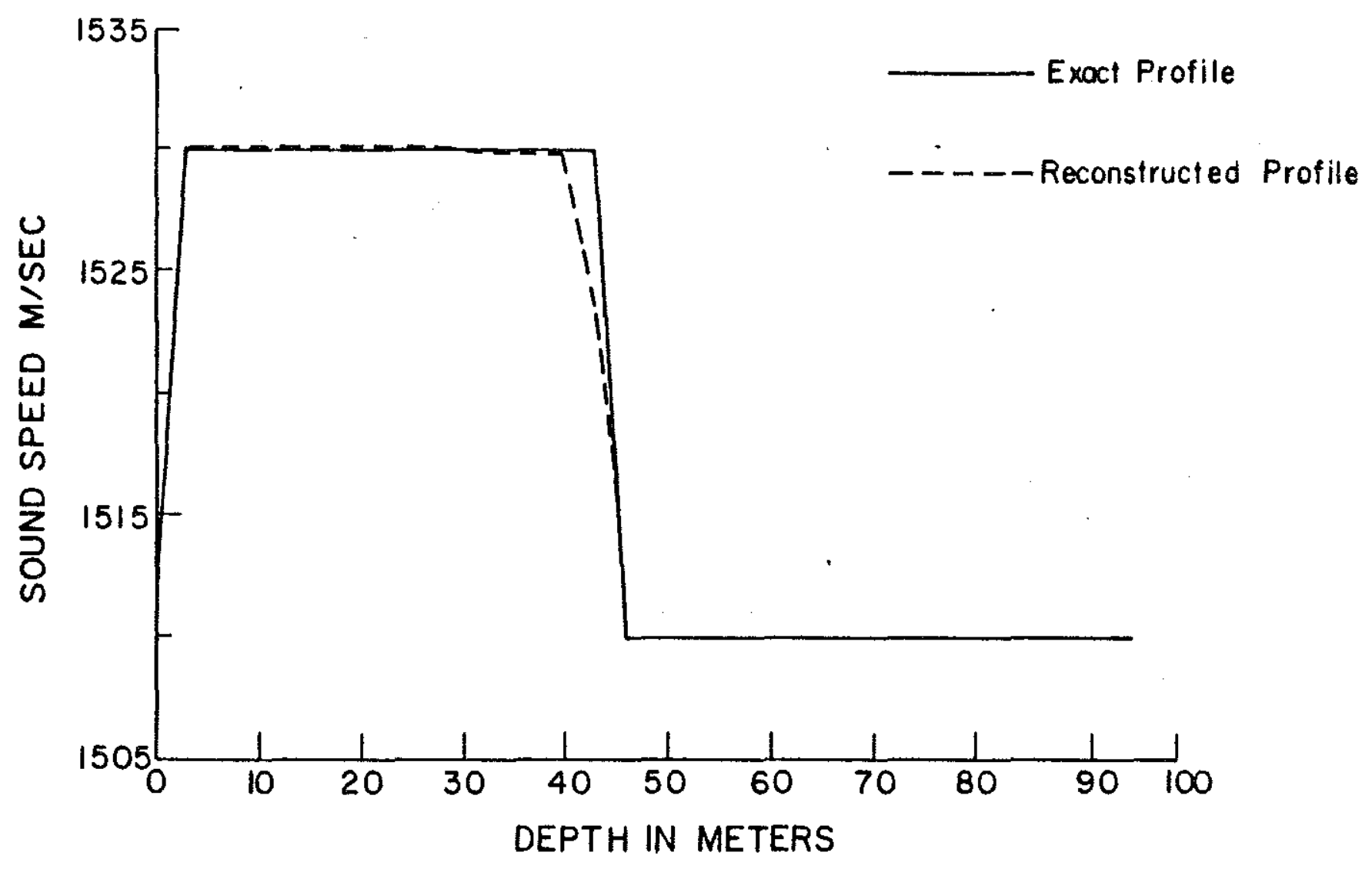

Figure 7-33: Reconstruction for lossless model using Gelfand-Levitan method 


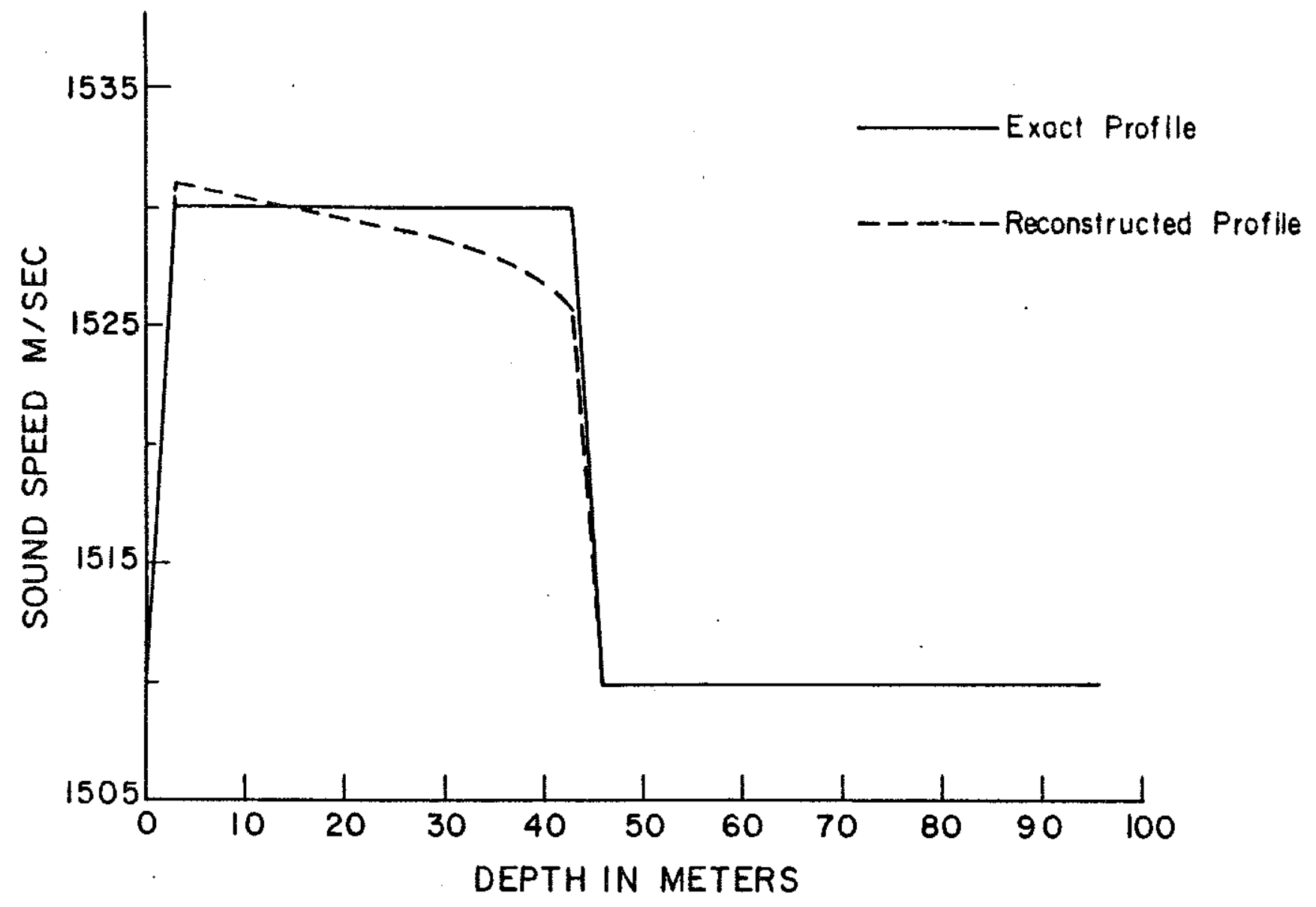

Figure 7-34: Reconstruction of lossymodel using Gelfand-Levitan method 
input data corresponding to the lossless case with every other parameter in the model remaining the same. The lossy and lossless models used are shown in Figure 7-32. The results of inversion is given in Figures 7-33 and 7-34. Even with the attenuation coefficient as small as $0.001 \mathrm{~dB} / \mathrm{m}$ there is observable error in the reconstructed value of the sound speed. However, the result obtained can be used as the initial guess model for the sound speed. We will call such errors in our knowledge of sound speed and density in the sediment as model errors and show how such errors can be dealt with.

The second form of errors are those in the data. The data, i.e, the planewave reflection coefficients are not measured directly but are obtained by suitably processing some other measured quantity. For the scheme proposed in Chapter 3 the directly measured quantity is the pressure field and this is then processed. Errors that occur in the measurement of this quantity will therefore be propagated and will finally appear as error in the reflection coefficient. We will call these data errors and show by way of examples how these affect the reconstruction of the attenuation profile.

\subsubsection{Errors in the model}

To show the effect of errors in the sound speed and density we use the model in Table 7-I. The input data is generated using the correct value of the sound speed. However while performing the inversion a slightly different value of sound speed is assumed. A similar test is done for error in density as well. The degradation that occurs in the solution is shown in Figures 7-35 and 7-36. Table 7-V gives the true sound speed and the value assumed for reconstruction. In the case of density the constant value of $1.7 \mathrm{gm} / \mathrm{cc}$ in the sediment layer is changed 1.75 $\mathrm{gm} / \mathrm{cc}$. In both cases the attenuation profile used for recontruction is in Figure 


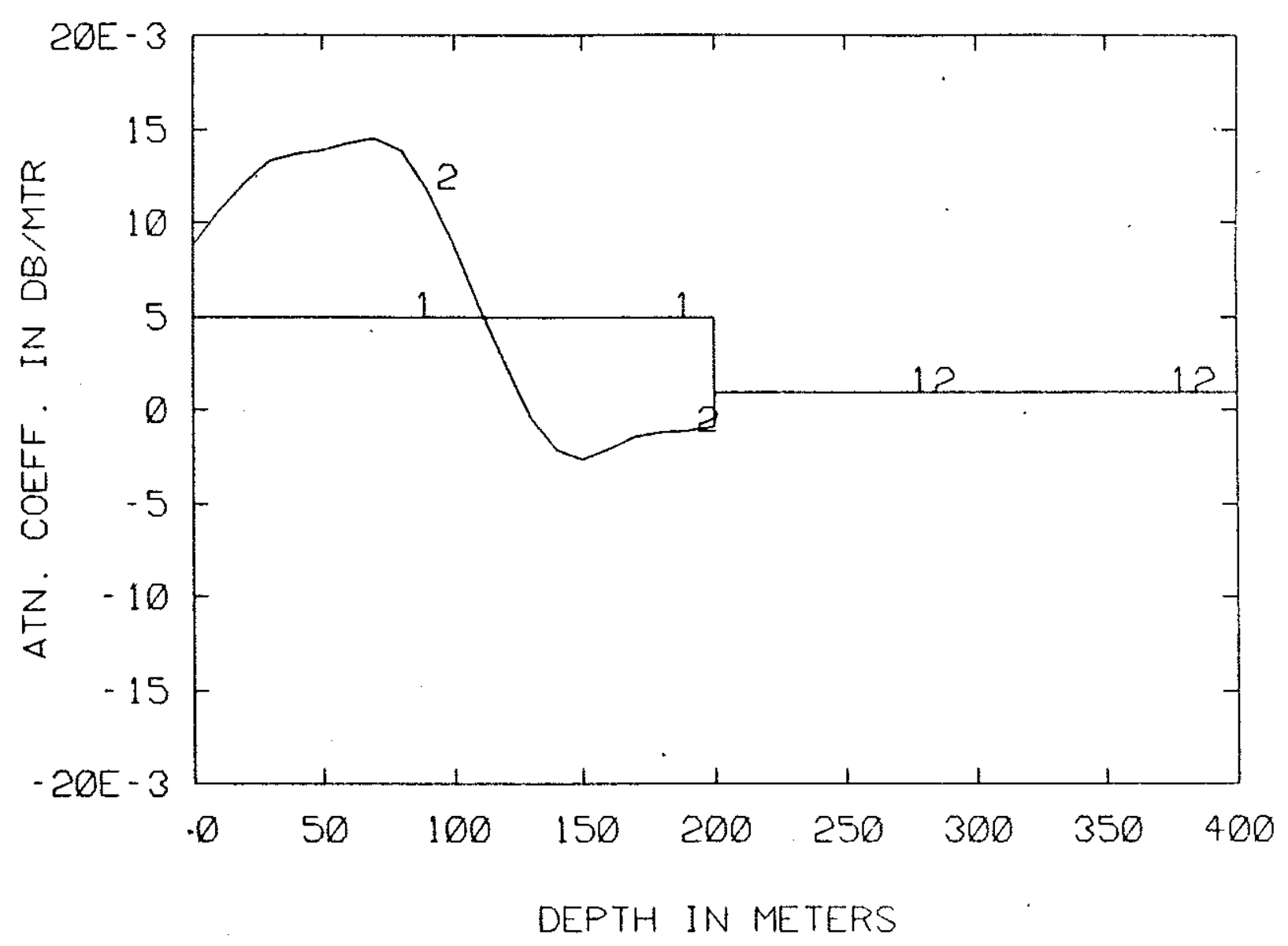

Figure 7-35: Effect of error in sound speed on reconstruction 1- True profile, 2- Reconstructed profile 
$-188-$

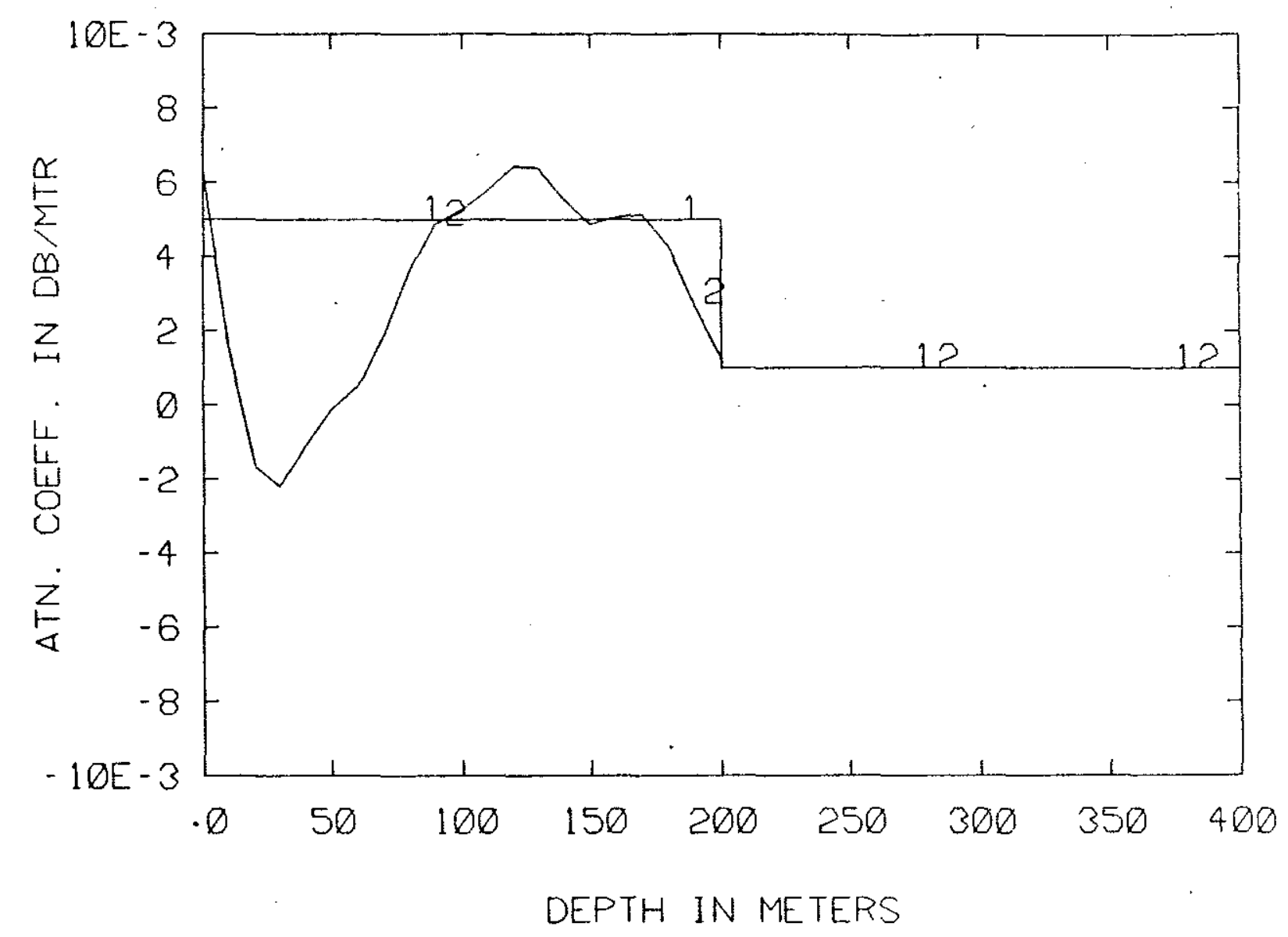

Figure 7-36: Effect of error in density on reconstruction

1- True profile, 2- Reconstructed profile 
$-198$.

\begin{tabular}{|c|c|c|c|c|c|}
\hline & & $\ldots$ & $\begin{array}{l}\text { Exact value of sound } \\
\text { speed in sediment }\end{array}$ & $\begin{array}{l}\text { Erroneous value } \\
\text { assumed. }\end{array}$ & $\begin{array}{l}\text { Water Interface } \\
\text { sediment } z=0\end{array}$ \\
\hline & Layer 1 & $10 \mathrm{~m}$ & 1517.56 & 1511.0 & . \\
\hline & Layer 2 & $20 \mathrm{~m}$ & 1530.39 & 1523.0 & \\
\hline & Layer 3 & $30 \mathrm{~m}$ & 1543.14 & 1535.0 & \\
\hline & Layer 4 & $40 \mathrm{~m}$ & 1555.74 & 1547.0 & \\
\hline & Layer 5 & $50 \mathrm{~m}$ & 1508.20 & 1559.0 & \\
\hline & Layer 6 & $60 \mathrm{~m}$ & 1580.52 & 1571.0 & \\
\hline depth & Layer 7 & $70 \mathrm{~m}$ & 1592.69 & 1583.00 & \\
\hline & Layer 8 & $80 \mathrm{~m}$ & 1604.74 & 1595.00 & \\
\hline & Layer 9 & $90 \mathrm{~m}$ & $\$ 616.64$ & 1607.00 & \\
\hline & Layer 10 & $100 \mathrm{~m}$ & 1628.41 & 1619.00 & \\
\hline & Layer 11 & $110 \mathrm{~m}$ & 1640.00 & 1631.00 & \\
\hline & Layer 12 & $120 \mathrm{~m}$ & 1651.55 & 1643.00 & \\
\hline & Layer 13 & $130 \mathrm{~m}$ & 1662.92 & 1655.00 & \\
\hline & Layer 14 & $140 \mathrm{~m}$ & 1674.16 & 1667.00 & \\
\hline & Layer 15 & $150 \mathrm{~m}$ & 1586.28 & 1679.00 & \\
\hline & Layer 16 & $160 \mathrm{~m}$ & 1696.27 & 1691.00 & \\
\hline & Layer 17 & $170 \mathrm{~m}$ & 1707.14 & 1703.00 & \\
\hline & Layer 18 & $180 \mathrm{~m}$ & 1717.88 & 1715.00 & \\
\hline & Layer 19 & $190 \mathrm{~m}$ & 1728.50 & 1727.00 & \\
\hline & Layer 20 & $200 \mathrm{~m}$ & 1739.00 & 1739.00 & Sediment Subbottom Intertace \\
\hline
\end{tabular}

Table 7-V: Exact and erroneous sound speed profile 
7-14(a).

Consider the case where there is an error in the background model for the sound speed profile. We can, then, relate the wavenumber of the true model to the wavenumber of the guess model by the expression given below.

$$
\begin{aligned}
& k(z)=\frac{\omega}{C_{b}(z)}\left[1+\frac{\Delta C(z)}{C_{b}(z)}\right] \\
& k(z)=k_{b}+\delta k(z)
\end{aligned}
$$

Since $\delta k(z)$ is not known it cannot be subtracted out. An alternative approach is to treat this as an unknown and invert to obtain $\delta k(z)+i \alpha(z)$.

We now take up the general case where we consider errors in the sound speed and density profiles of the guess model. The parameters for the true model and the background model are now related by the following equation.

$$
\begin{aligned}
& k(z)=k_{b}(z)+\delta k(z)+i \alpha(z) \\
& \rho(z)=\rho_{b}(z)+\beta(z)
\end{aligned}
$$

Our aim is to obtain an integral equation which on inversion will yield the corrections to the sound speed and density profiles in addition to the attenuation coefficent profile. To do this we make the assumption that the density profile and its first derivative are continuous across the interfaces. We, then, use the procedure developed in Chapter 5 to derive the integral equation for this case. Using equations (5.3) and (5.4), we express $\mu(\mathrm{z})$ as a sum of $\mu_{\mathrm{b}}(\mathrm{z})$ and a perturbation of it as given by the following equation.

$$
\mu(z)=\mu_{b}(z)+\delta(z)
$$


where,

$$
2 \rho_{b}^{2}(z) \delta(z)=\frac{d^{2} \beta(z)}{d z^{2}}+3 \frac{d \rho_{b}(z)}{d z} \frac{d \beta(z)}{d z}-\left[\frac{d^{2} \rho_{b}(z)}{d z^{2}}+3 \frac{d \rho(z)}{d z}\right] \beta(z)
$$

The integral equation then is,

$$
\begin{gathered}
i k_{z 0}\left\{R_{b}\left(k_{x}-R\left(k_{x}\right)\right\}=\int_{0}^{h} \frac{1}{\rho_{b}(z)}\left[2 k_{b}(z)(\delta k(z)+i \alpha(z))+\delta(z)\right]\right. \\
P_{b}^{2}\left(k_{x}, z\right) d z
\end{gathered}
$$

This equation can be solved to obtain $2 \mathrm{k}_{\mathrm{b}}(\mathrm{z}) \delta \mathrm{k}(\mathrm{z})+\delta(\mathrm{z})$ and $2 \mathrm{k}_{\mathrm{b}}(\mathrm{z}) \alpha(\mathrm{z})$. To seperate $\delta \mathrm{k}(\mathrm{z})$ and $\delta(\mathrm{z})$ we use the method due to Coen[14]. The experiment is performed at two frequencies and for each case the integral equation is solved. Let the solutions obtained be $\eta_{1}$ and $\eta_{2}$ respectively. Then the real part of the solutions can be expressed as follows.

$$
\begin{aligned}
& \eta_{1 r}(z)=2 \frac{\omega_{1}^{2}}{C_{b}^{3}(z)} \Delta C(z)+\delta(z) \\
& \eta_{2 r}(z)=2 \frac{\omega_{2}^{2}}{C_{b}^{3}(z)} \Delta C(z)+\delta(z)
\end{aligned}
$$

Subtracting equation (7.69) from (7.68), we obtain

$$
\Delta C(z)=\frac{C_{b}^{3}(z)}{2} \frac{\eta_{1 r}(z)-\eta_{2 r}(z)}{\omega_{1}{ }^{2}-\omega_{2}{ }^{2}}
$$

We obtain $\Delta \mathrm{C}(\mathrm{z})$ and $\delta(\mathrm{z})$ from the above. Having obtained $\delta(\mathrm{z})$ we then obtain $\beta(\mathrm{z})$ by using the equation (7.66). The boundary condtions for the problem are $\beta(0)=0$ and $\beta^{\prime}(0)=0$.

Now consider the case when there is only error in the sound speed. In 
equation (7.65) we set $\delta(z)=0$. We then obtain the integral equation given below.

$$
i k_{z 0}\left\{R\left(k_{x}\right)-R_{b}\left(k_{x}\right)\right\}=\int_{0}^{h k_{b}(z)} \frac{\rho_{b}(z)}{\delta}\{k(z)+i \alpha(z)\} P_{b}^{2}\left(k_{x}, z\right) d z
$$

For discrete values of $k_{x}$ we obtain,

$$
d_{n}=\int_{0}^{h} m(z) G_{n}(z) d z
$$

where

$$
\begin{aligned}
& d_{n}=i\left\{k_{z 0}\left(R-R_{b}\right)\right\}_{n}=\left(d_{r}\right)_{n}+i\left(d_{i}\right)_{n} \\
& G_{n}(z)=G_{n r}(z)+i G_{n i}(z) \\
& m(z)=m_{r}(z)+i m_{i}(z)
\end{aligned}
$$

with $G_{n r}(z)$ and $G_{n i}(z)$ being the real and imaginary parts of $P_{b}{ }^{2}\left(k_{n}, z\right)$. If the data are available at discrete data points, we we employ a quadrature scheme and obtain a matrix equation of the form given below.

$$
\mathbf{d}=\mathbf{G m}
$$

where $\mathbf{d}$ is the vector $\left[\mathbf{d}_{\mathbf{r}}, \mathbf{d}_{\mathbf{i}}\right]^{\mathbf{T}}, \mathbf{m}$ is the vector $\left[\mathbf{m}_{\mathbf{r}}, \mathbf{m}_{\mathbf{i}}\right]^{\mathbf{T}}$ and $\mathbf{G}$ is the matrix given below.

$$
G=\left[\begin{array}{ll}
G_{r} & -G_{i} \\
G_{i} & G_{r}
\end{array}\right]
$$

To solve this matrix equation we again use the regularisation procedure and impose constraints on the solution based on smoothness criteria for the two 


\begin{tabular}{|c|c|c|c|c|}
\hline & $\begin{array}{c}\text { Exact } \\
\text { Soundspeed } \mathrm{m} / \mathrm{s}\end{array}$ & $\begin{array}{l}\text { initial } \\
\text { Guess value m's }\end{array}$ & $\begin{array}{l}\text { Exact } \\
\mathrm{dB} / \mathrm{m}\end{array}$ & $\begin{array}{c}\text { Initial guess } \\
\mathrm{dB} / \mathrm{m}\end{array}$ \\
\hline \multirow{2}{*}{$10 \mathrm{~m}$} & 1517.56 & 1511.00 & 0.005 & 0.0 \\
\hline & 1530.39 & 1523.00 & 0.005 & 0.0 \\
\hline $20 \mathrm{~m}$ & 1543.14 & 1535.00 & 0.005 & 0.0 \\
\hline $30 \mathrm{~m}$ & 1555.74 & 1547.00 & 0.005 & 0.0 \\
\hline $40 \mathrm{~m}$ & 1568.20 & 1559.00 & 0.005 & 0.0 \\
\hline $50 \mathrm{~m}$ & 1580.52 & 1571.00 & 0.005 & 0.0 \\
\hline $60 \mathrm{~m}$ & 1592.69 & 1583.00 & 0.005 & 0.0 \\
\hline $70 \mathrm{~m}$ & 1604.74 & 1595.00 & 0.005 & 0.0 \\
\hline $80 \mathrm{~m}$ & 1616.64 & 1607.00 & 0.005 & 0.0 \\
\hline $90 \mathrm{~m}$ & 1628.41 & 1619.00 & 0.005 & 0.0 \\
\hline $100 \mathrm{~m}$ & 1640.40 & 1631.00 & 0.005 & 0.0 \\
\hline $110 \mathrm{~m}$ & 1651.55 & 1643.00 & 0.005 & 0.0 \\
\hline $120 \mathrm{~m}$ & 1662.92 & 1655.00 & 0.005 & 0.0 \\
\hline $130 \mathrm{~m}$ & 1674.16 & 1667.00 & 0.005 & 0.0 \\
\hline $140 \mathrm{~m}$ & 1685.28 & 1679.00 & 0.005 & 0.0 \\
\hline $150 \mathrm{~m}$ & 1696.27 & 1691.00 & 0.005 & 0.0 \\
\hline $160 \mathrm{~m}$ & 1707.14 & 1703.00 & 0.005 & 0.0 \\
\hline $170 \mathrm{~m}$ & 1717.88 & 1715.00 & 0.005 & 0.0 \\
\hline $180 \mathrm{~m}$ & 1728.50 & 1727.00 & 0.005 & 0.0 \\
\hline $190 \mathrm{~m}$ & 1739.00 & 1739.00 & 0.005 & 0.0 \\
\hline
\end{tabular}

Table 7-VI: True and background model parameters - case(i) 
$-204-$

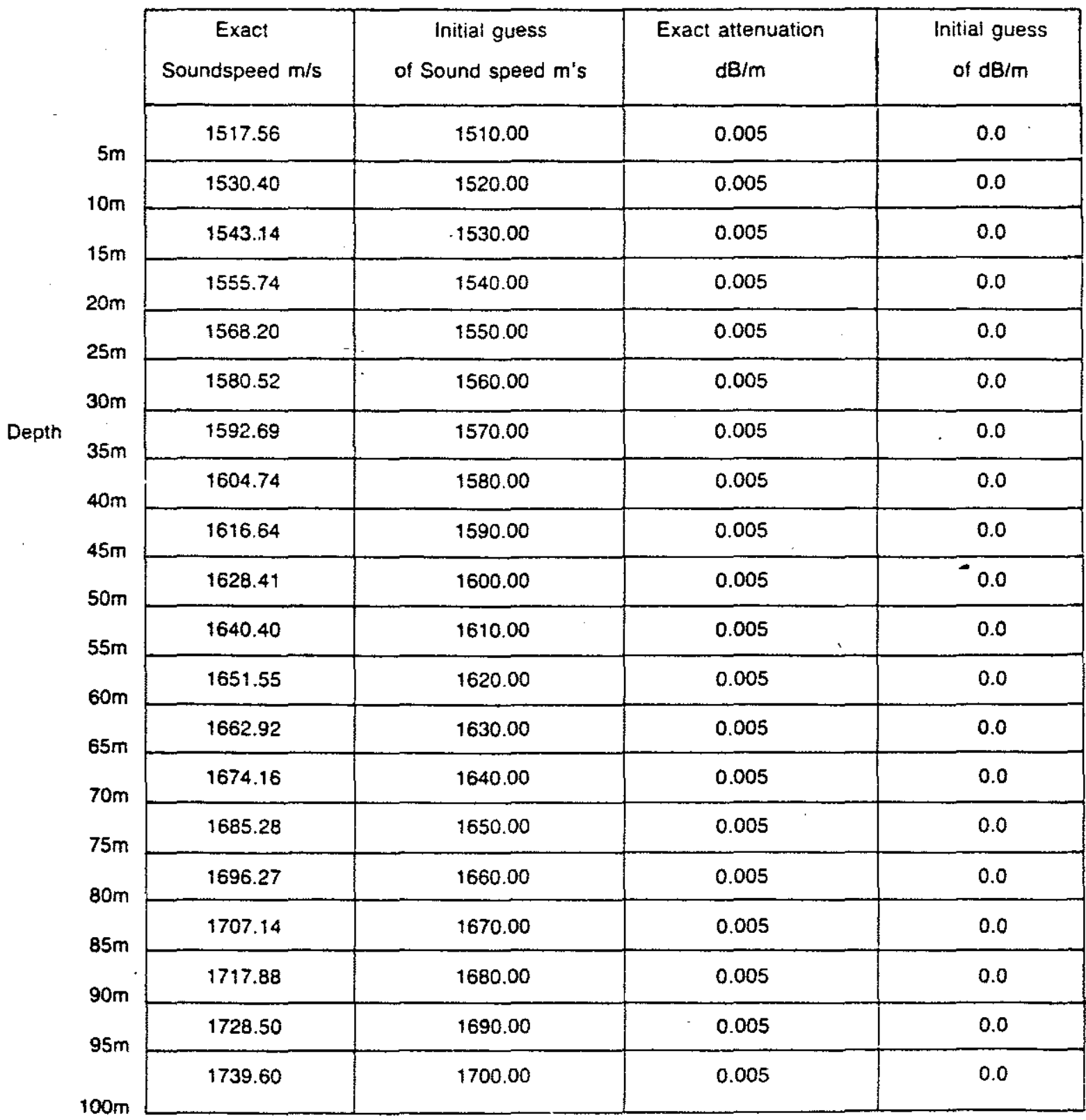

Table 7-VII: True and background model parameters - case(ii) 


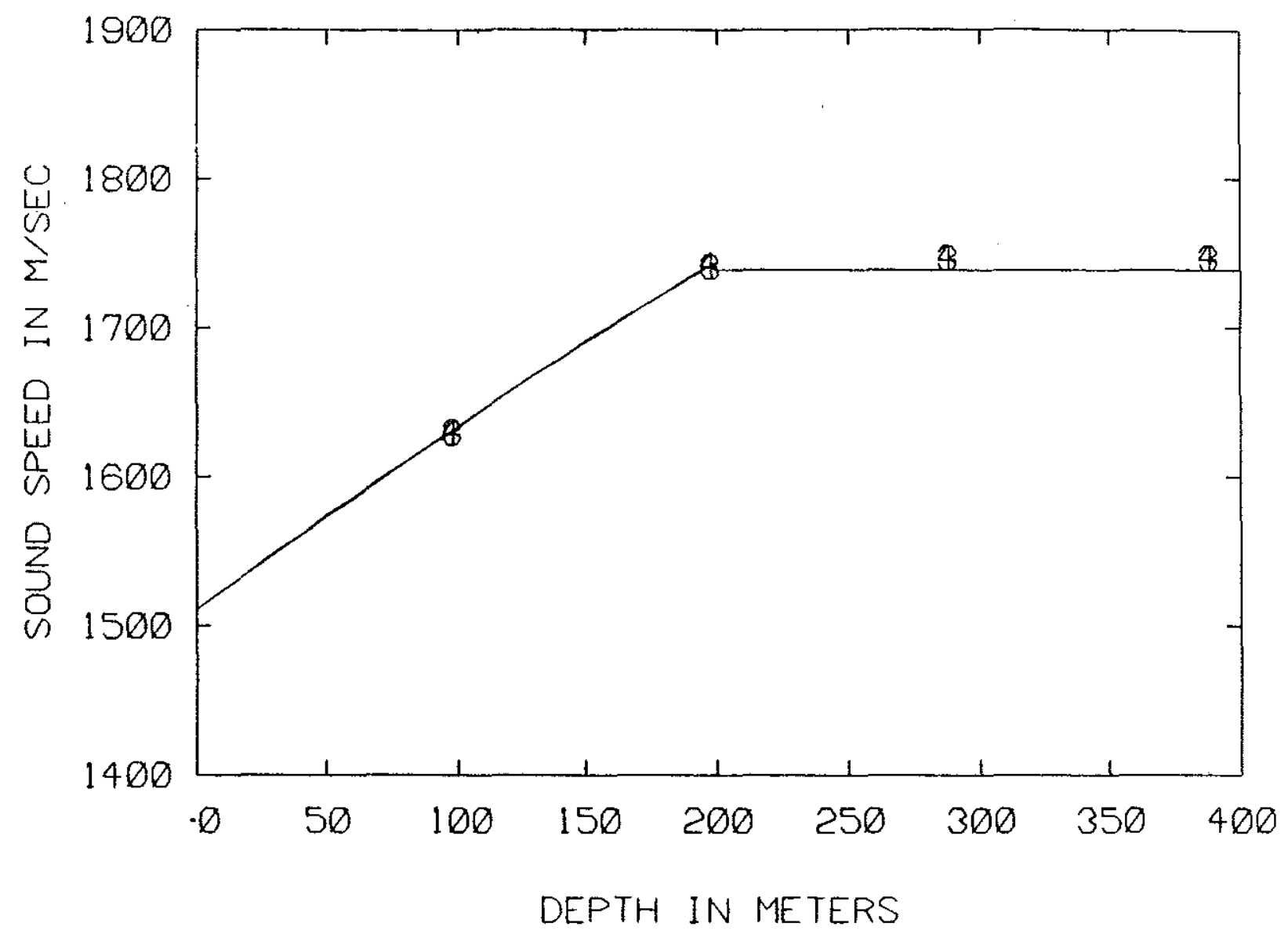

Figure 7-37: Simultaneous reconstruction - sound speed profile; case (i) 3- True profile, 4- Reconstructed profile 
$-206-$

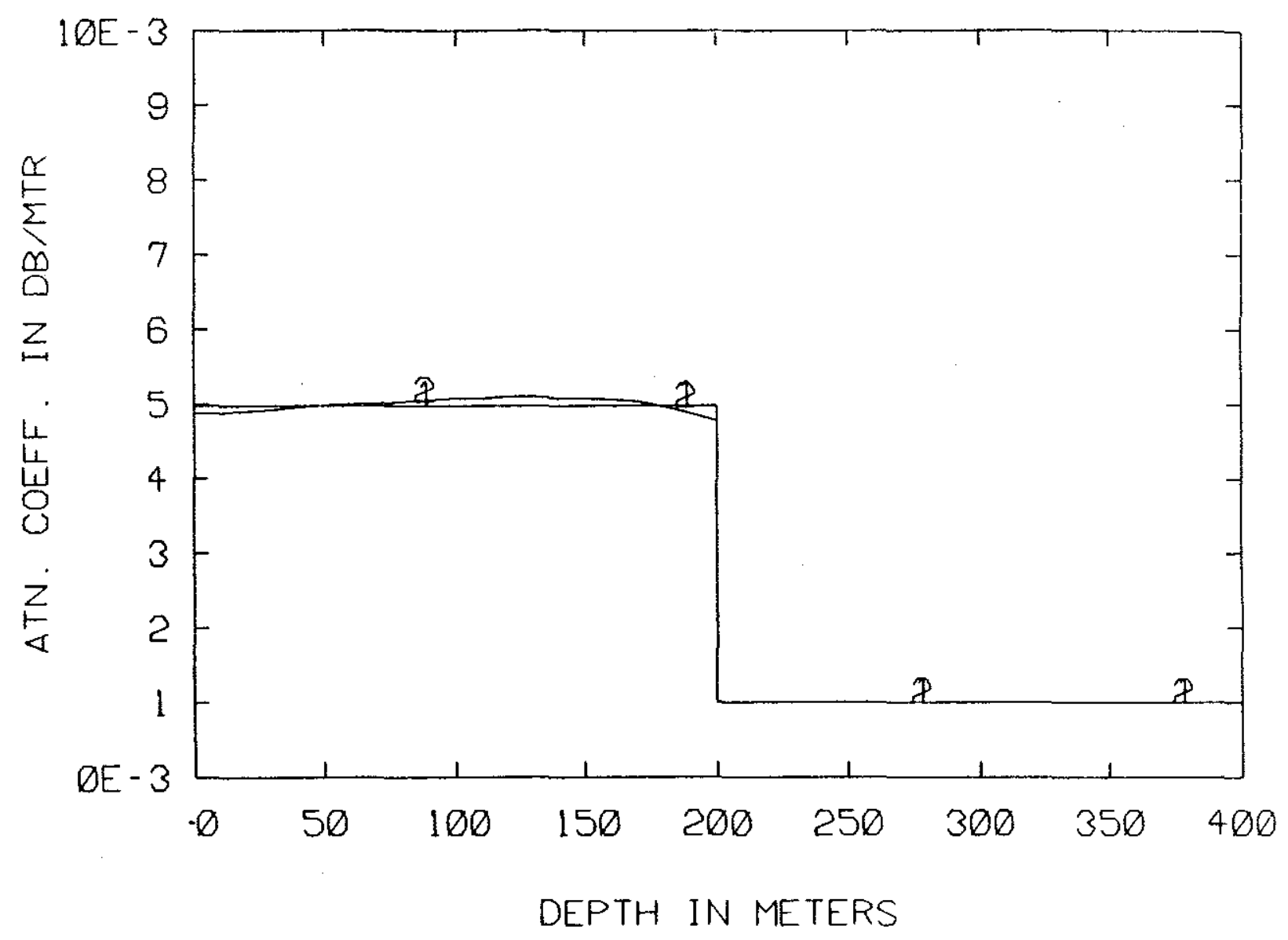

Figure 7-38: Simultaneous reconstruction - Attenuation profile; case(i) 1- True profile, 2- Reconstructed profile 


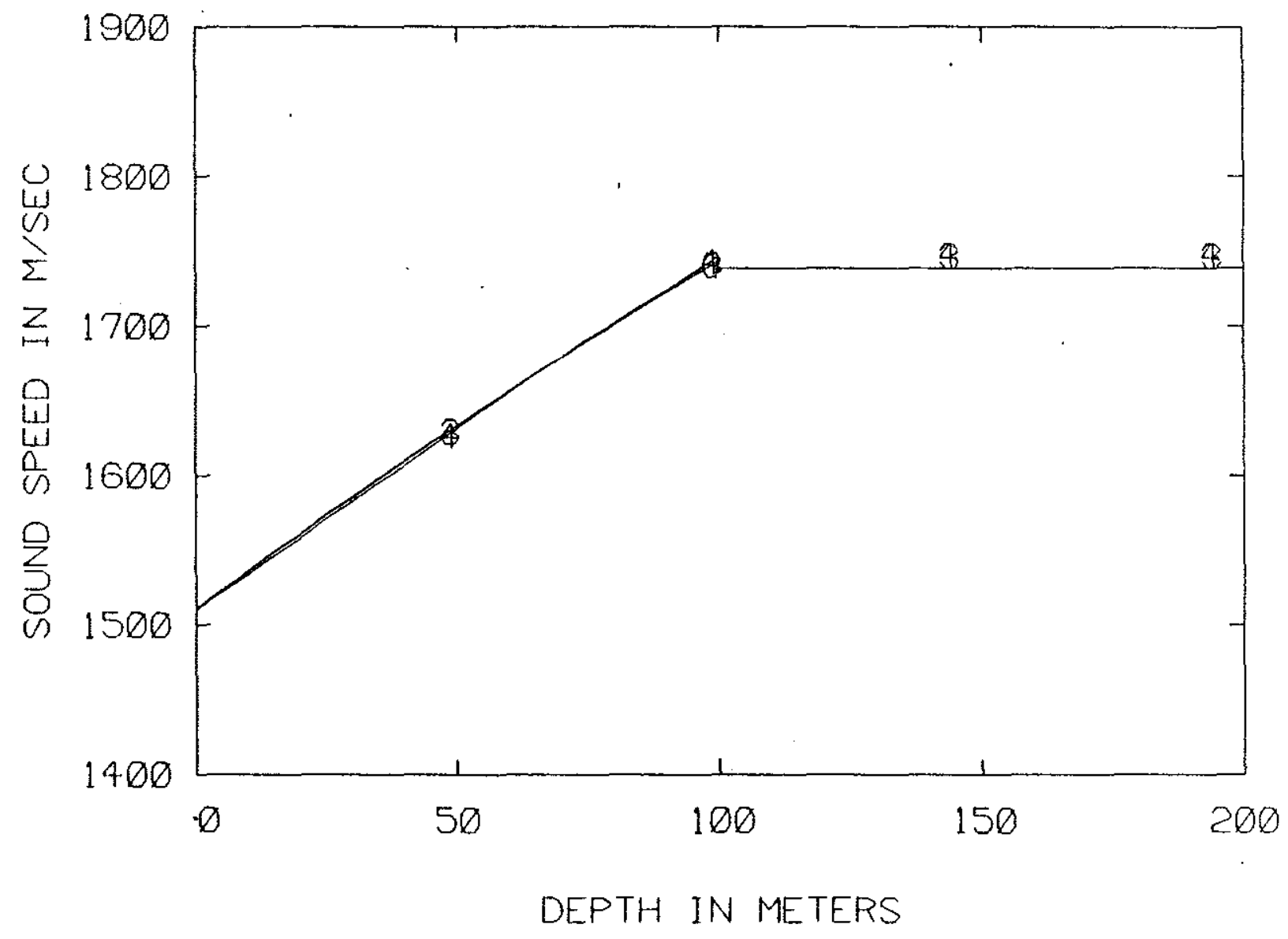

Figure 7-39: Simultaneous reconstruction - Sound speed profile; case(ii) 3- True profile, 4- Reconstructed profile 


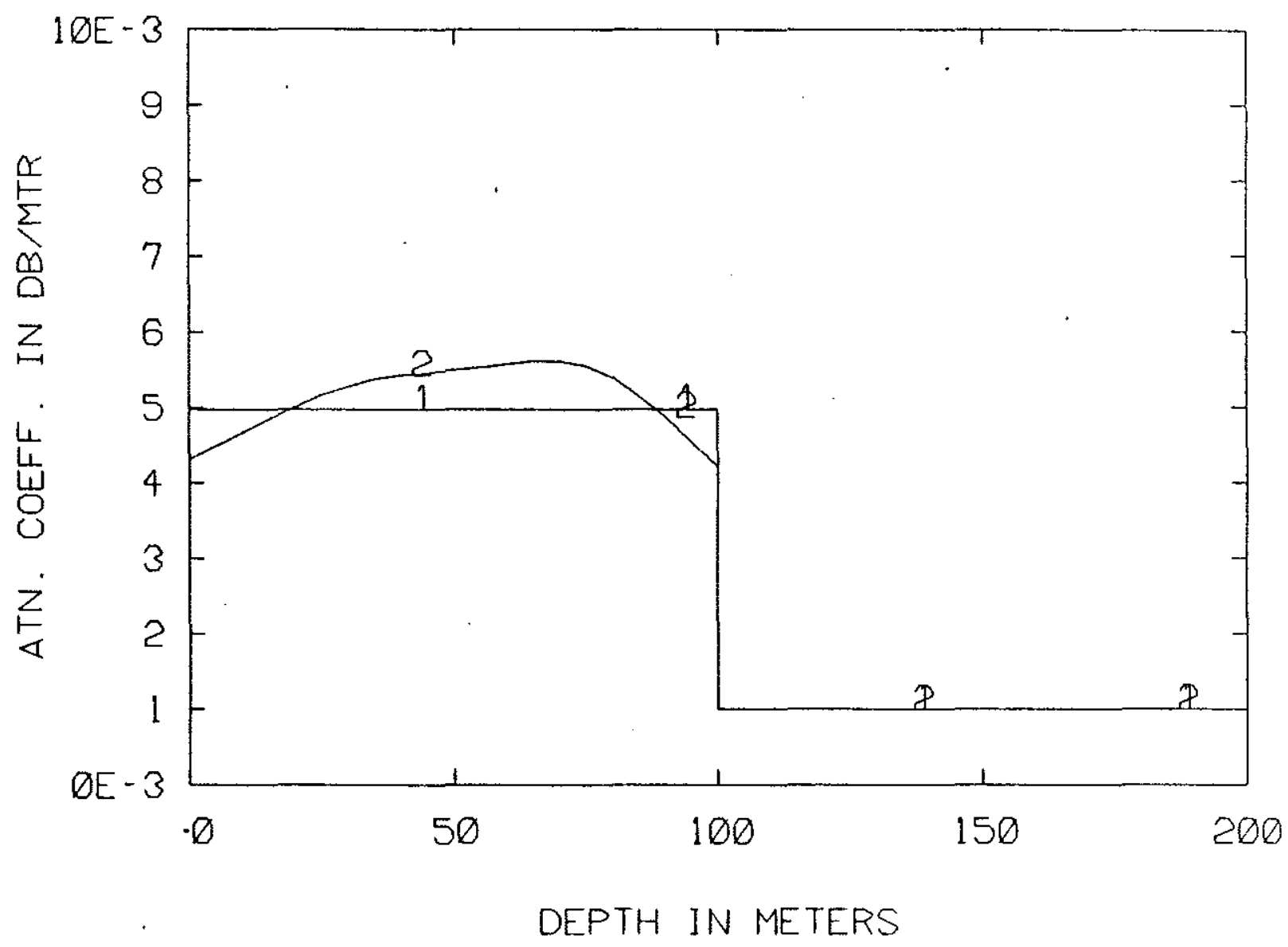

Figure 7-40: Simultaneous reconstruction - Attenuation profile; case(ii) 1- True profile, 2- Reconstructed profile 
$-209-$

functions being reconstructed. We, therefore, seek to minimise the sum of the smoothness measures subject to the data constraints being met. The solution then takes the form

$$
\left[\mathrm{G}^{T} \mathbf{G}+\lambda\left(\mathbf{H}_{1}+\mathrm{H}_{2}\right)\right] \mathbf{m}=\mathbf{G}^{T} \mathbf{d}
$$

where $\mathrm{H}_{1}$ and $\mathrm{H}_{2}$ will depend oh the smoothness measure for the functions.

Using such a scheme, simultaneous reconstruction of the sound speed and attenuation profile was done. Tables 7-VI and 7-VII give the details of the exact and initial guess values for the two cases considered. The exact.and reconstructed profiles are in Figures 7-37 to 7-40.

While considering the effect of frequency on the inversion for the attenuation profile we stated that the frequency has no effect on inversion. However, when simultaneous inversion is performed the perturbation associated with it is the magnitude of $\delta \mathrm{k}(\mathrm{z})+\mathrm{i} \alpha(\mathrm{z})$. Since $\delta \mathrm{k}$ is propotional to the square of frequency, the product $\delta \mathrm{kG}\left(\mathrm{z}, \mathrm{z}^{\prime}\right)$ will be propotional to frequency. This leads us to conclude that in this case a lower frquency is better for reconstruction.

The case where there are errors in our knowledge of the subbottom parameters is treated in Appendix B

\subsubsection{Errors in data}

We have already mentioned that to model the error in the data we will need to know the proceesing that has been done to obtain the data, namely, the plane wave reflection coefficients. In the scheme envisaged in Chapter 3 the first stage is to obtain the Green's function by carrying out a Hankel transform operation of the

pressure field. Mook[16] studied the effect of adding stationary white gaussian noise 


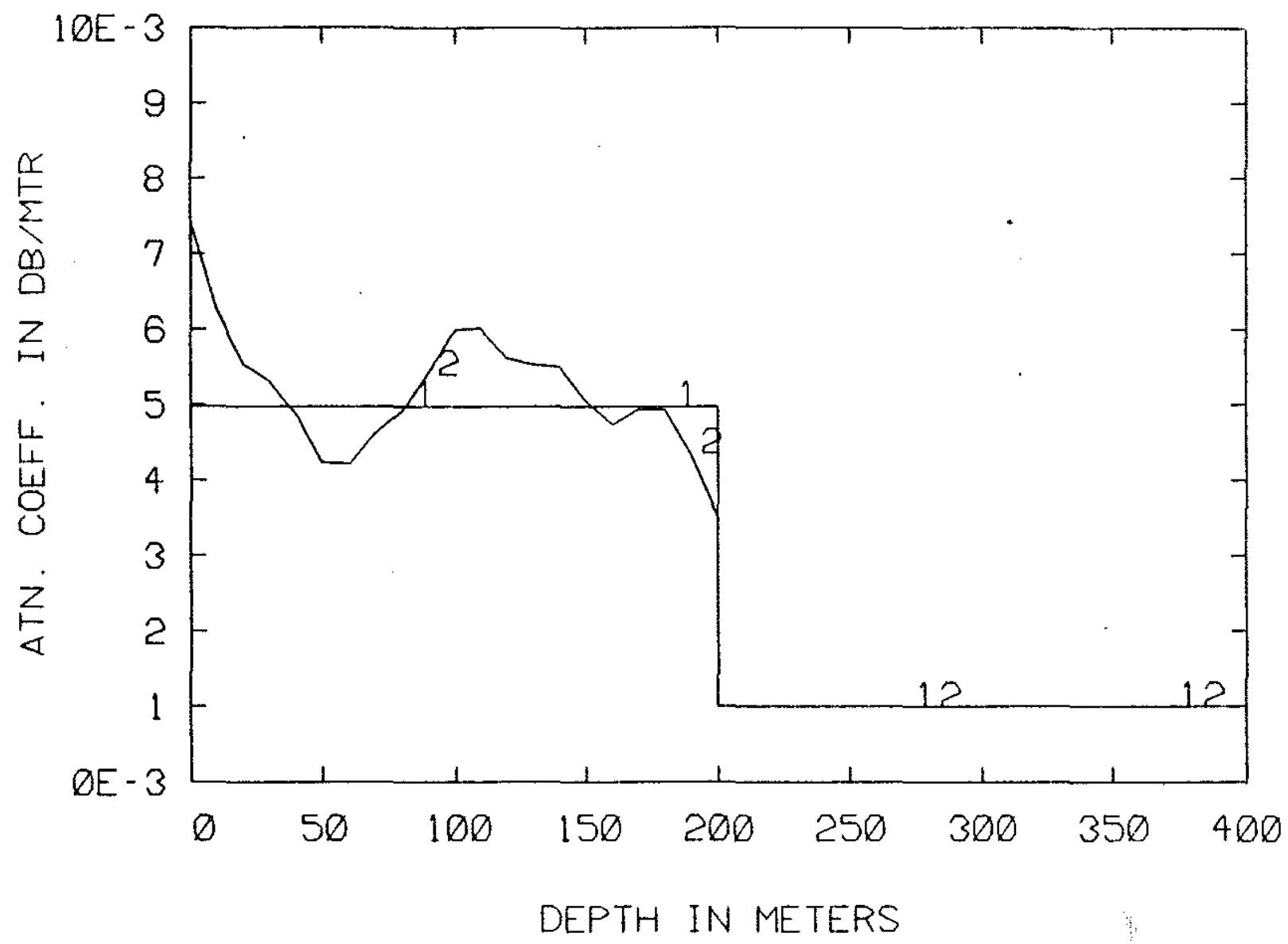

Figure 7-41: Reconstruction of profile in figure 7-14(a) with noisy data-40dB

1- True profile, 2- Reconstructed profile 
$-211-$

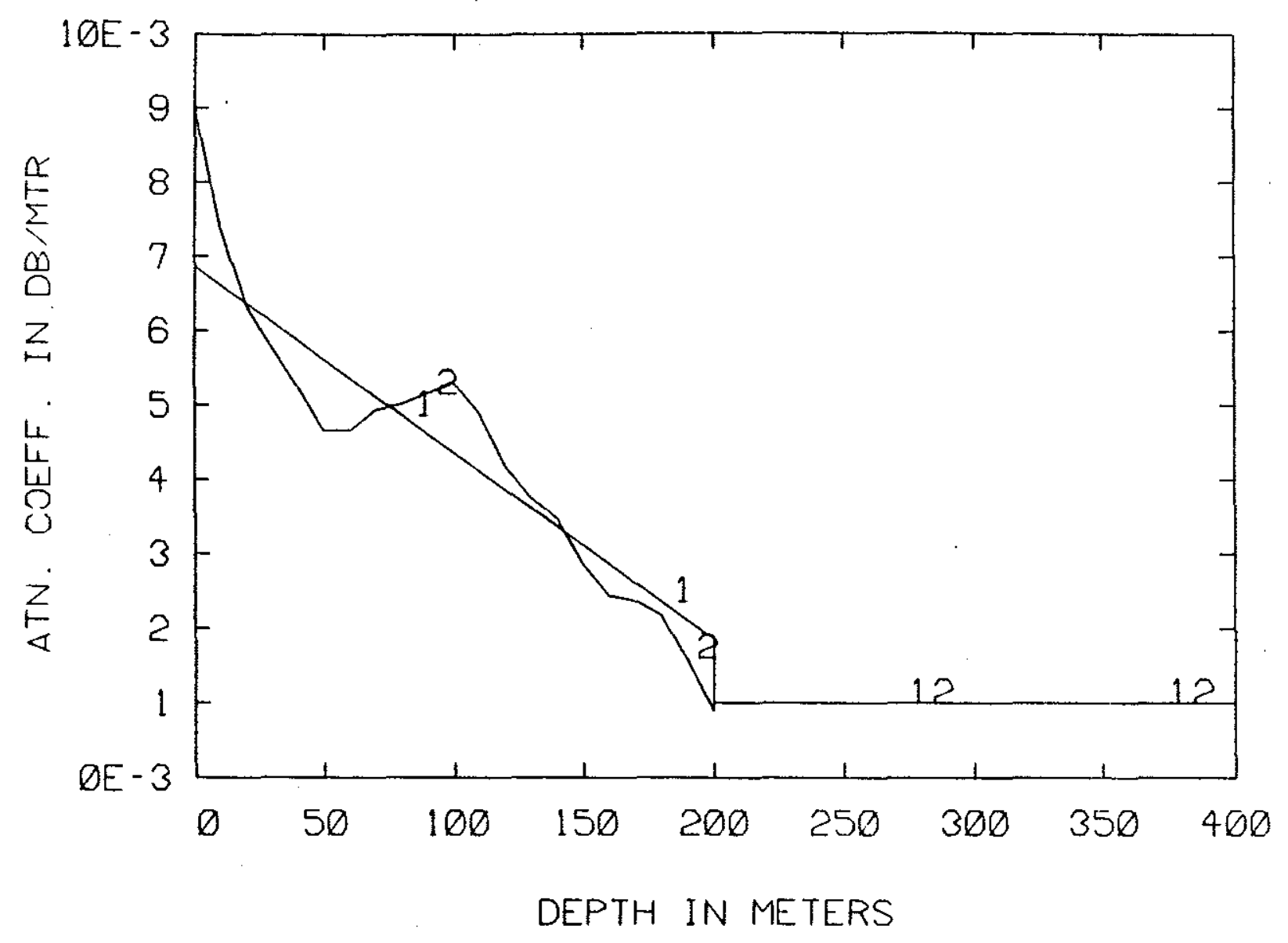

Figure 7-42: Reconstruction of profile in figure 7-14(b) with noisy data $-40 \mathrm{~dB}$

1- True profile, 2-Reconstructed profile 


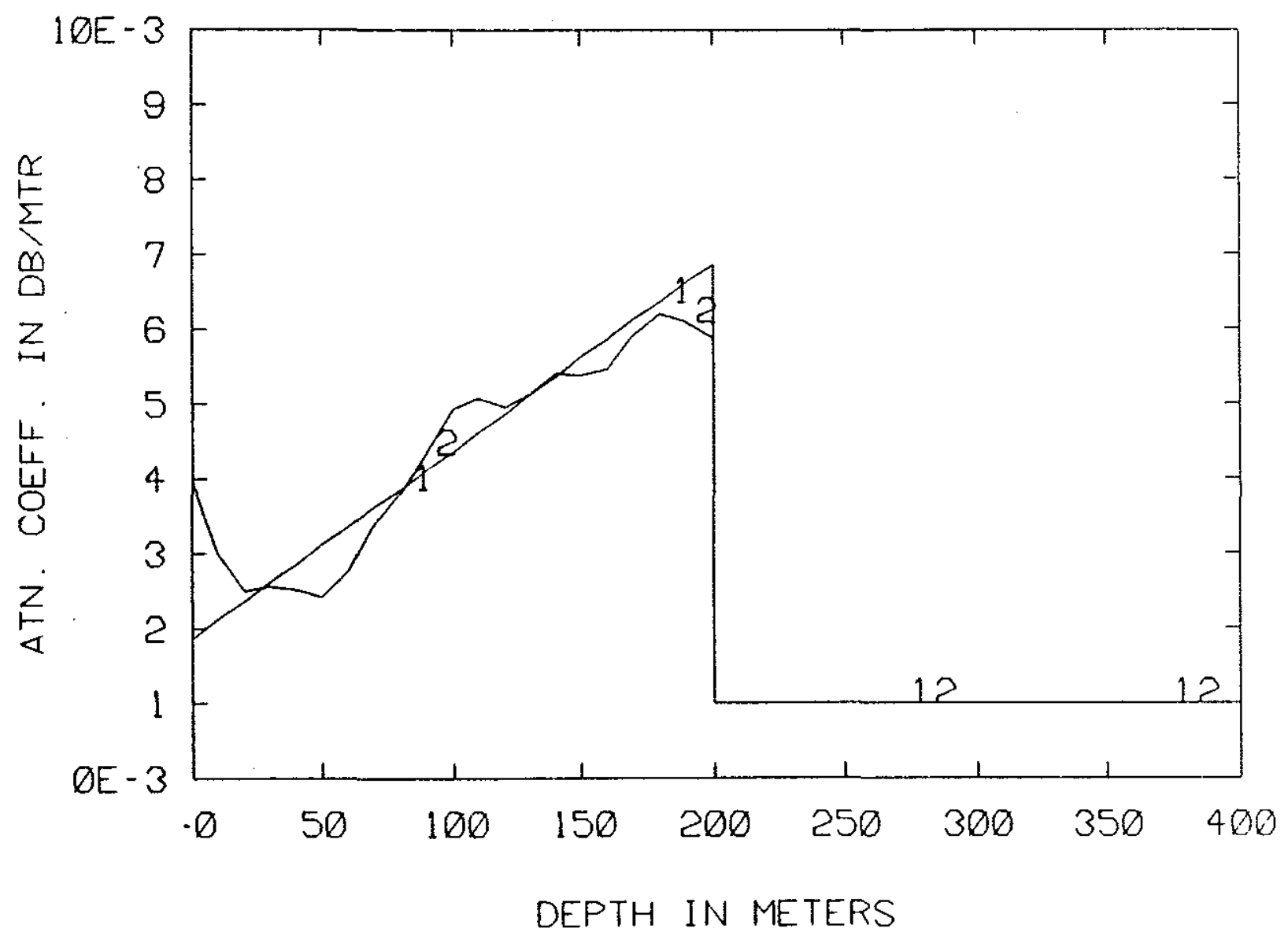

Figure 7-43: Reconstruction of profile in figure 7-14(c) with noisy data- $40 \mathrm{~dB}$ 1- True profile, 2-Reconstructed profile 


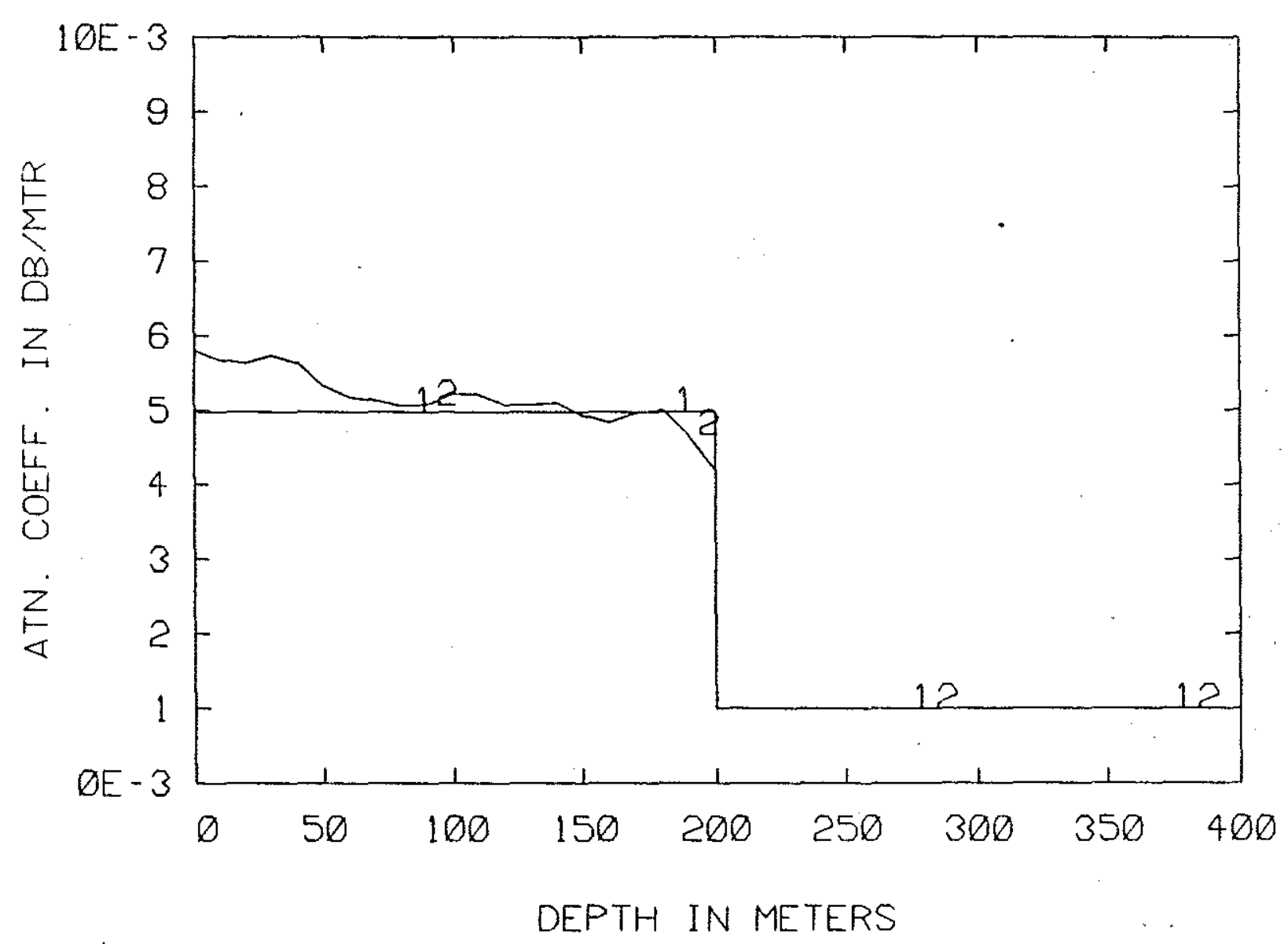

Figure 7-44: Reconstruction of profile in figure 7-14(a) with noisy dat a $50 \mathrm{~dB}$

1- True profile, 2- Reconstructed profile 


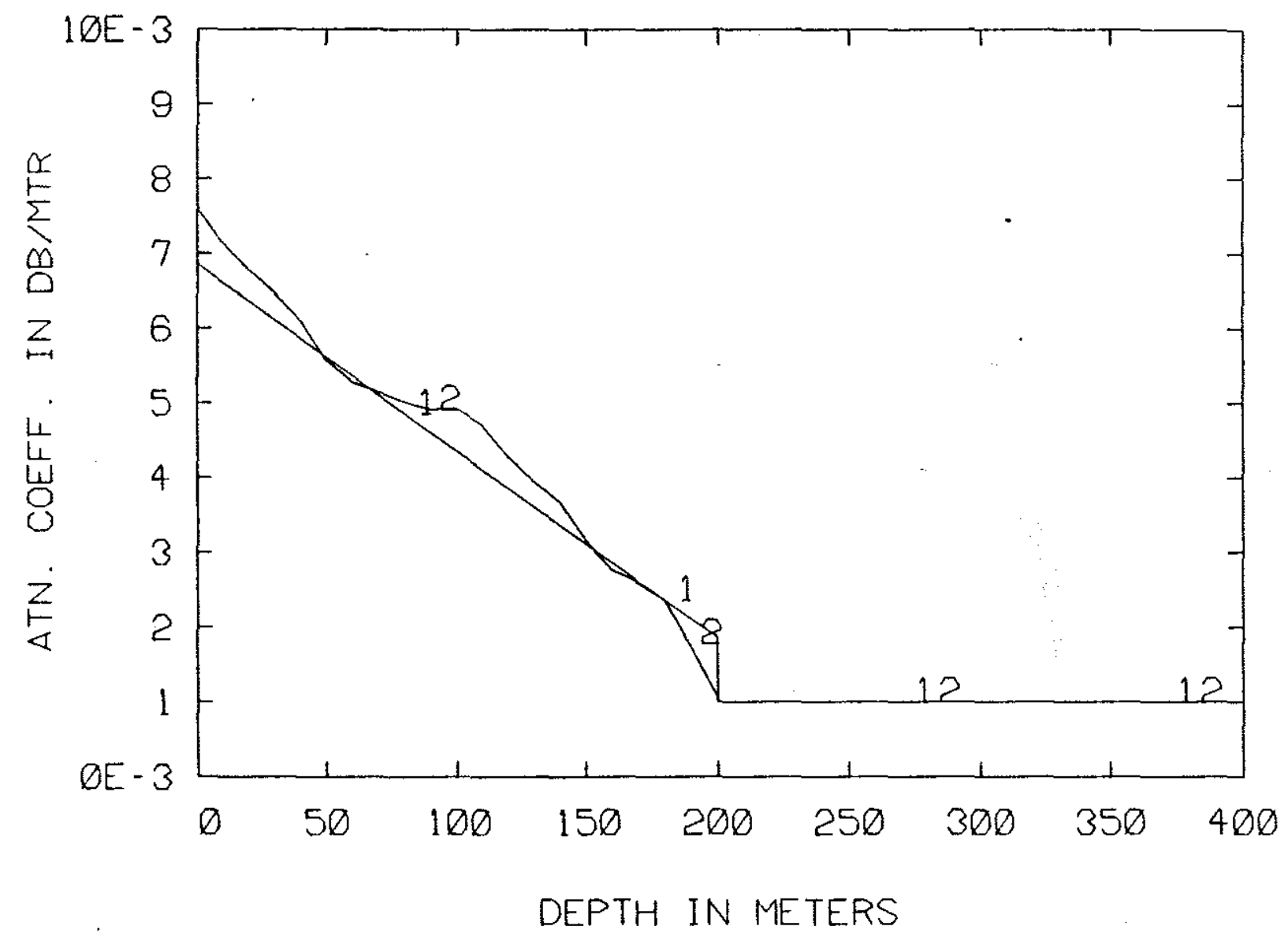

Figure 7-45: Reconstruction of profile in figure 7-14(b) with noisy data $50 \mathrm{~dB}$

1. True profile, 2-Reconstructed profile 
$-215-$

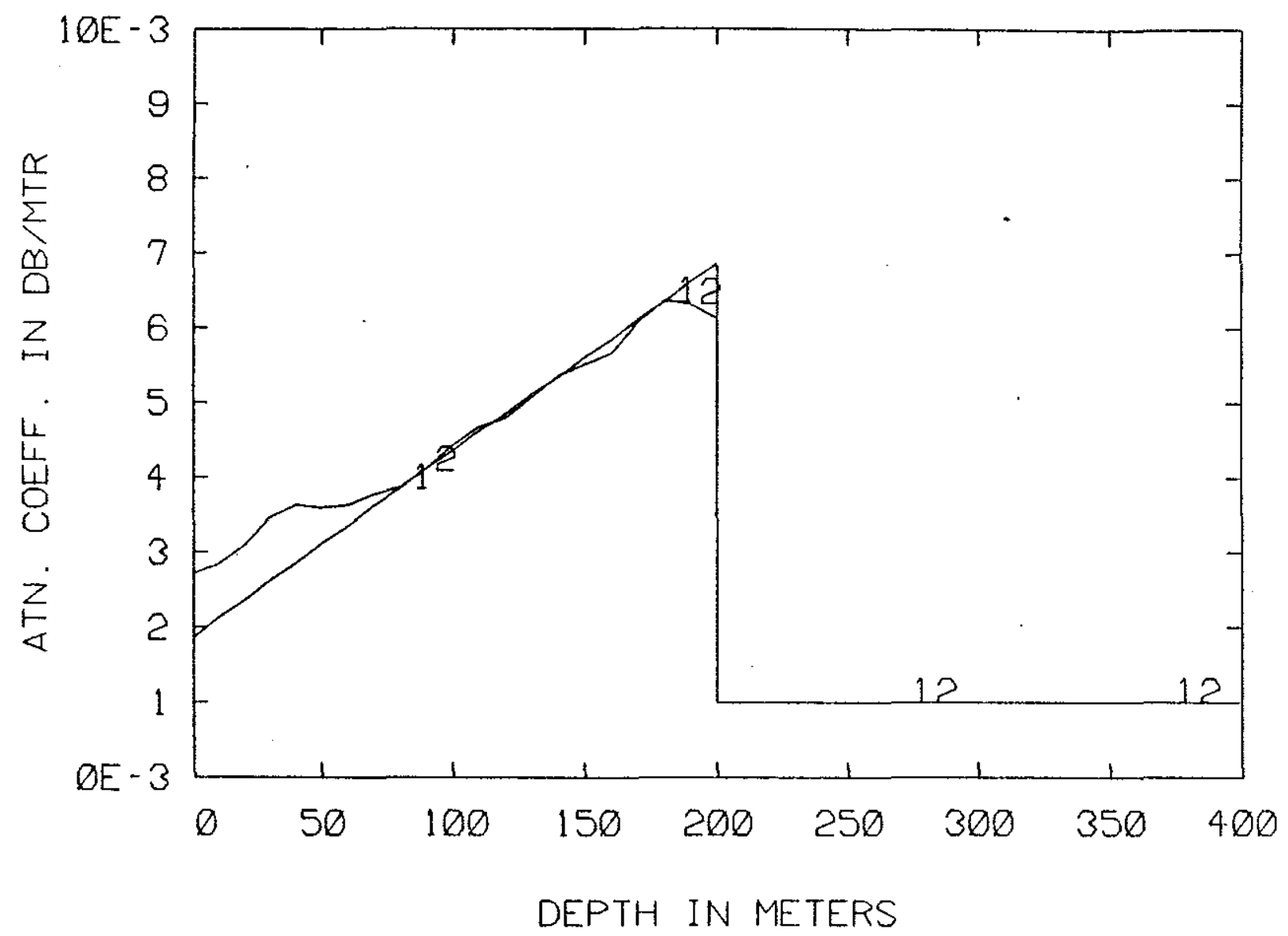

Figure 7-46: Reconstruction of profile in figure 7-14(c) with noisy data $50 \mathrm{~dB}$ 
to the pressure field. If the noise is zero mean, the expected value of the output of the Hankel transform will not be corrupted since the Hankel transform operation is a linear one. However the variance is found to be non-stationary and the noise power is concentrated around $k_{r}=0$ where $k_{r}$ is the horizontal wavenumber. The non stationarity of the noise is simply that it is dependent on $k_{r}$ value and this makes it difficult to model noise in the reflection coefficient. However we make the assumption that within the small angular aperture that we use for the data that the noise is stationary and gaussian. With this assumption we define signal to noise ratio as given below.

$$
S N R=10 \log _{10}\left\{\frac{I_{s}}{I_{n}}\right\}
$$

For reflection coefficients at a set of $\mathrm{N}$ discrete points we have

$$
\begin{aligned}
& I_{s}=\frac{1}{N_{n=1}^{N}\left|R_{n}\right|^{2}} \\
& I_{n}=\sigma_{n}{ }^{2}
\end{aligned}
$$

Using this measure, errors corresponding to $40 \mathrm{~dB}$ and $50 \mathrm{~dB}$ was added to each data point and inversion of the attenuation profile carried for diiferent type of profiles. The results obtained for the different cases are shown in Figures 7-41 to 7-46. This experiment was performed to assess whether the inversion method is stable in the presence of noise. We note that for the noise levels used, the scheme is stable and yields reasonable results. 


\subsection{Summary}

In this chapter we demonstrated that the linear integral ecuation derived in Chapter 5 can be solved to obtain the attenuation coefficient profile for the ocean bottom. Though we started with the problem of requiring to determine the imaginary part of the wavenumber, a perturbation of the real part, we showed that the scheme can yield perturbations in both the real and imaginary part thereby enabling determination of all the three acoustic parameters. We demonstrated this by determining the attenuation coefficient profile and the sound speed profile. We

studied the effect of experimental and acoustic parameters on inversion. The resolving power theory of Backus and Gilbert[1] was used to assess the resolution obtainable with the data and examined the effect of the angular aperture and the number of data points on inversion. Finally we showed by examples that the inversion method is stable in the presence of noise.

In the next chapter, we examine briefly another approach to obtain the acoustic parameters of the ocean bottom which is suitable in the shallow water context. 


\subsection{References}

1. G. Backus and F. Gilbert,"The resolving pwer of gross earth data," Geophys. J. R. Astr. Soc., 16, 169-205 (1968).

2. R.L. Parker, "The Frechet derivative for the one dimensional electromagnetic induction problem," Geophys. J. R. Astr. Soc., 49, 543-547 (1977).

3. B. Carnahan, H.A. Luther, and J.O. Wilkes, Applied numerical methods, (Wiley, New York, 1969).

4. S. Twomey, Introduction to the mathematics of inversion in remote sensing and indirect measurements, (Elsevier, New York, 1977).

5. A. Roger, "Determination of the index profile of a dielectric plate from a scattering data," in Applied Inverse Problems,(Springer-Verlag, New York, 1978).

6. D.W. Marquardt, "An algorithm for least squared estimation of nonlinear parameters," J. Soc. Indust. Applied Math., 11(2), 431-441 (1963).

7. K. Miller, and G.A. Vilano, "On the necessity of nearly best possible methods for the analytic continuation of scattering data," J. Math Physics, 14, 1037-1048 (1873).

8. E.L. Hamilton, "Geoacoustic modelling of the sea floor," J. Acoust. Soc. Am., 68(5), 1313-1340 (1980).

9. G.V. Frisk, D.R. Mook, J.A. Doutt, E.E. Hays, and A.V. Oppenheim, "The application to real data of a technique for measuring the plane wave reflection coefficient of the ocean bottom, "J. Acoust. Soc. Am., 72(S1)S97, (1982).

10. J.B. Keller, "Accuracy and validity of the Born and Rytov approximation, " J. Opt. Soc. Am., 59, 1003-1004 (1969).

11. R.K. Muller, M. Kaveh, and G. Wade,"Reconstructive tomography and applications to ultrasonics," Proceeding of the IEEE, 67(4), 567-587 (1979). 
12. M. Kaveh, R.K. Muller, and R.D. Iverson, "Ultrasonic tomography based on perturbation solution of the wave equation, "Computer Graphics and Image Processing, 9, 105-116 (1979).

13. R.L. Parker, "Understanding inverse theory," Ann. Rev. Planet. Sci., 5, 35-64 (1977).

14. S. Coen, K.K. Mei, and D.J. Anglakos, "Inverse scattering technique applied to remote sensing of a layered media," IELE Trans. Antennas Propagat., AP-29(2), 298-306 (1981).

15. A. Merab, "Reconstruction of the ocean bottom velocity profiles from monochromatic scattering data," Sc.D. thesis, M.I.T/W.H.O.I Joint Program, Cambridge, MA. and Woods Hole, MA. (June1985).

16. D.R. Mook, "The numerical synthesis and inversion of acoustic fields using the Hankel transform with application to the estimation of the plane wave reflection coefficient of the ocean bottom," Sc.D. thesis, M.I.T/W.H.O.I Joint Program, Cambridge,MA., and Woods Hole, MA. (January, 1983). 


\section{Chapter 8 \\ Acoustic Parameters from Eigenvalues}

In this chapter we will show that in the shallow water context a first order perturbation theory can be used to connect perturbations in the modal eigenvalues to the acoustical properties of the ocean bottom. Such a formulation leads to an integral equation of the type we have considered so far in earlier chapters and therefore the procedures recommended for the solution of such equations can be used here as well.

Frisk and Lynch[1] have proposed that an experiment similar to the one described in Chapter 3 can be carried out in the shallow water and measurement of the pressure field taken. The experimental configuration is shown in Figure 8-1. If the pressure field obtained is now Hankel-transformed to obtain the Green's function, distinct peaks can be observed at the modal eigenvalues. This has been demonstrated by Frisk and Lynch[2] using real data. Figure 8-2 is the Greens function computed by carrying out the Hankel transform of the pressure field measured in an experiment conducted off the Nantucket sound. The position of the poles of the Green's function are easily seen. These occur at the modal eigenvalues.

If the medium is absorbing the modal eigenvalues will become complex and the real and imagimary parts of the eigenvalues need to be found. Frisk and Lynch[1] give a method by which this can be done. We, therefore, assume that from the measurements of the pressure field an estimate of the modal eigenvalues have been obtained. The eigenvalues of the modes depend on the acoustic parameters of

the bottom and therefore have this information. The problem then is to develop a 
TYPICAL SHALLOW WATER EXPERIMENTAL CONFIGURATION

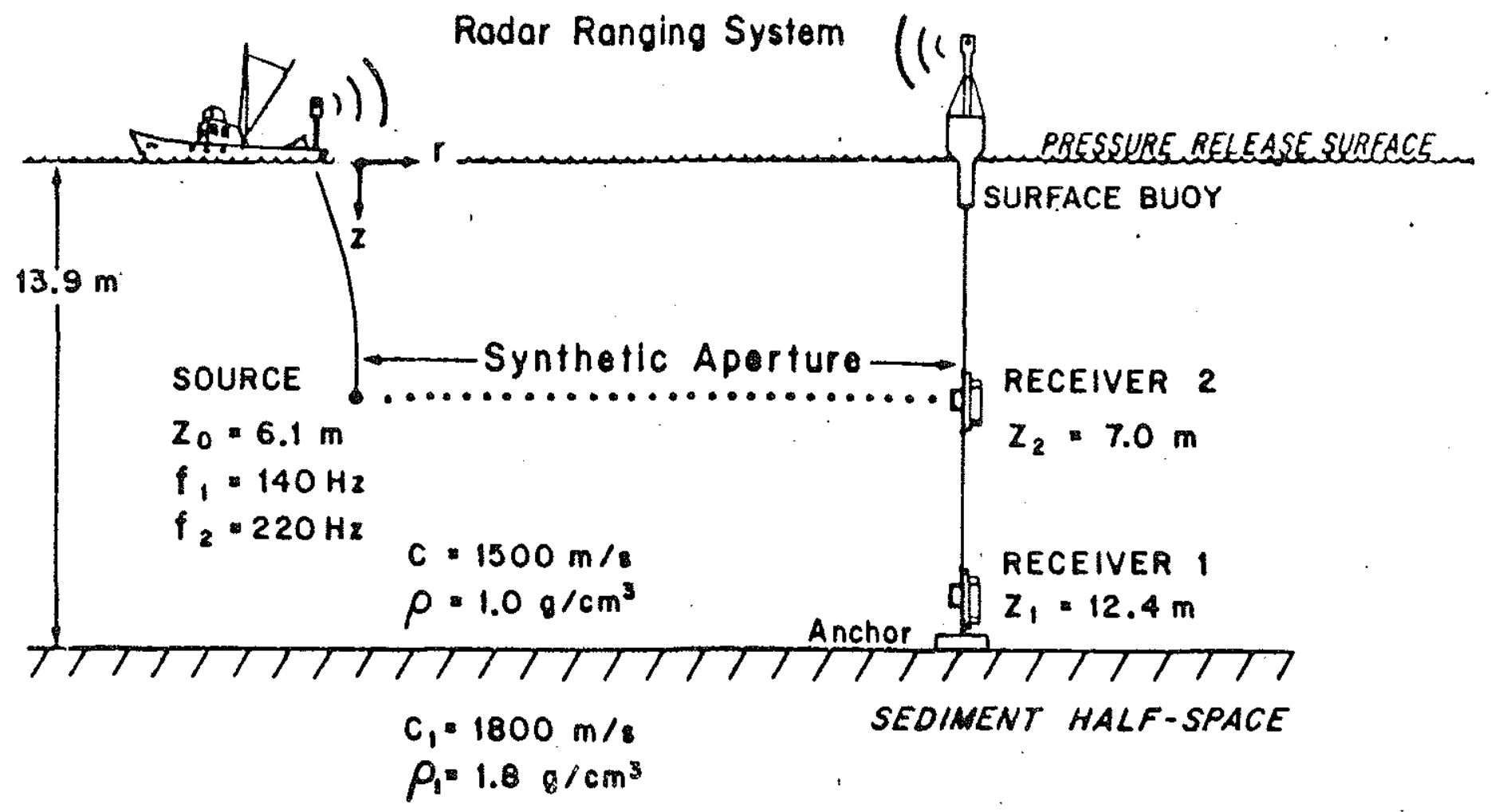

Figure 8-1: Experimental configuration for shallow water experiment 


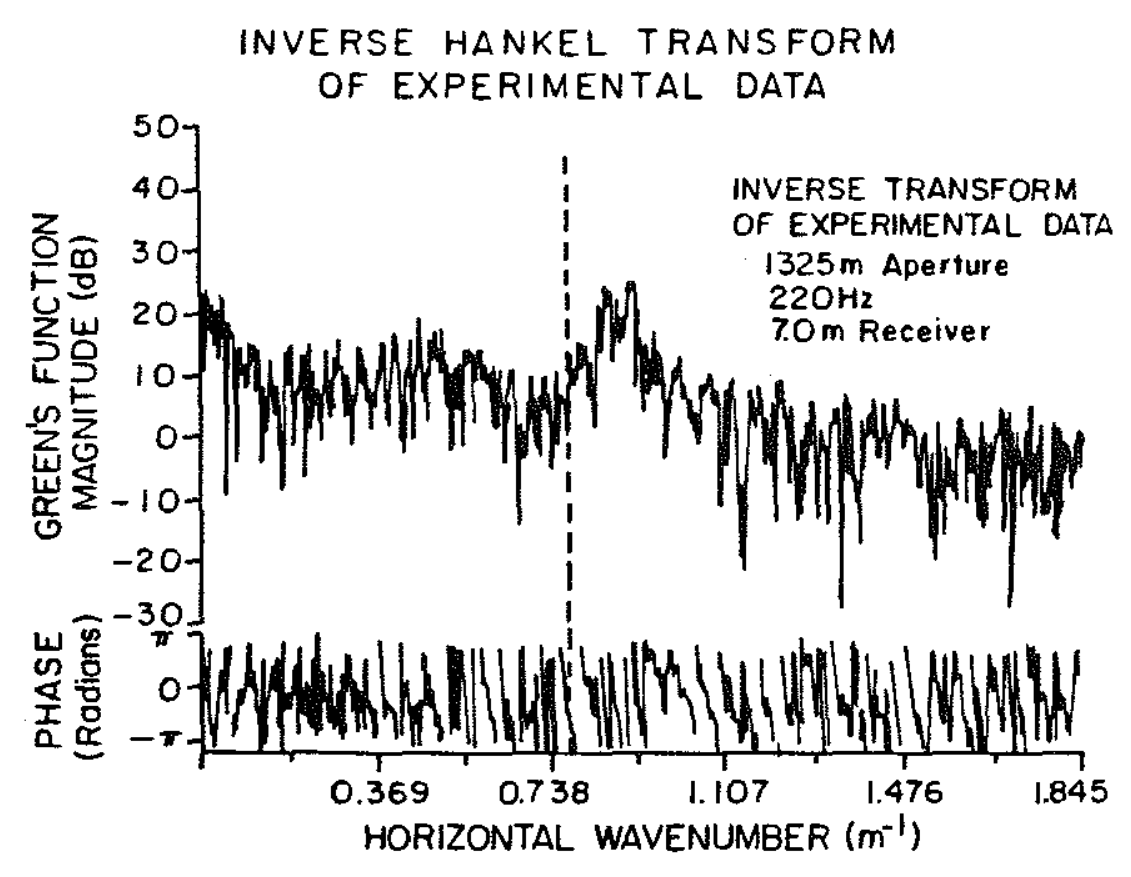

Figure 8-2: Hankel transform of measured pressure field 
procedure to reconstruct the geoacoustic parameters of the bottom from a knowledge of the modal eigenvalues.

\subsection{Derivation of integral equation}

Consider the model shown in Figure 8-3. In the shallow water environment we assume that the sound speed in the water column is constant and all the acoustical parameters in the water column are known. We are now required to determine the density, sound speed and attenuation profiles for the bottom.

We assume that from archival data or other sources of information we are able to get approximate values for these parameters. This is used as the initial guess model for the bottom and a perturbative approach used to construct an integral equation which will relate the changes in the eigenvalues between that of the guess model and the real model to the acoustic parameters of the bottom.

Making the assumptions of circular symmetry to a point source field and horizontal stratification, the equations for the normal mode is given by the following equation.

$$
\frac{d^{2} U_{n}(z)}{d z^{2}}+\rho(z)\left(\frac{1}{\rho(z)}\right)^{\prime} \frac{d U_{n}(z)}{d z}+\left(k^{2}(z)-k_{n}{ }^{2}\right) U_{n}(z)=0
$$

where $k(z)$ is the complex wave number for the medium, i.e absorption in the medium is accounted for by making the wave number complex. $k_{n}$ is the modal eigenvalue and $U_{n}$ the modal function for the nth mode. Making the substitution $v_{n}(z)=\rho^{-1 / 2}(z) U_{n}(z)$ we obtain,

$$
\frac{d^{2} v_{n}(z)}{d z^{2}}+\left(k^{2}(z)+\mu(z)-k_{n}^{2}\right) v_{n}(z)=0
$$




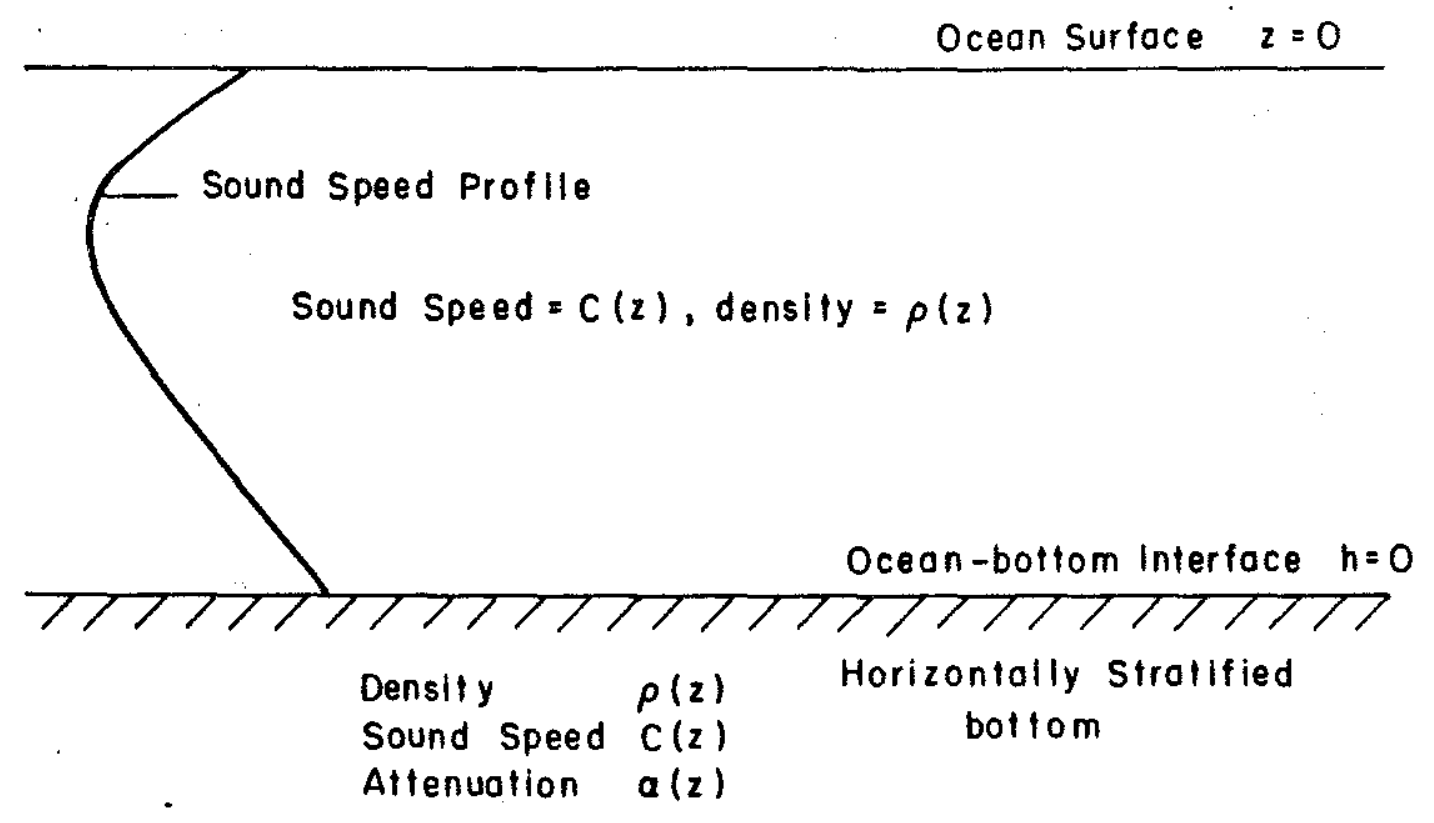

Figure 8-3: Ocean model 
where,

$$
\mu(z)=\frac{\rho^{1 / 2}(z)}{2}\left\{\frac{\rho^{\prime}(z)}{\rho^{3 / 2}(z)}\right\}^{\prime}
$$

Let $C_{b}(z)$ and $\rho_{b}(z)$ be the initial guess model for the ocean bottom. The attenuation is assumed to be zero for the guess model. Then,

$$
\frac{d^{2} v_{n b}(z)}{d z^{2}}+\left(k_{b}{ }^{2}(z)+\mu_{b}(z)-k_{x}{ }^{2}\right) v_{n b}(z)=0 .
$$

where,

$$
\mu_{b}=\frac{\rho_{b}^{1 / 2}}{2}\left\{\frac{\rho_{b}^{\prime}(z)}{\rho_{b}^{3 / 2}(z)}\right\}^{\prime}
$$

Let,

$$
v(z)=v_{n b}(z)+v_{n s}(z)
$$

and

$$
k(z)=k_{b}+i \alpha(z) ; \mu(z)=\mu_{b}(z)+\delta(z)
$$

Substituting the above into equation (8.2),we obtain

$$
\begin{array}{r}
v_{n b}{ }^{\prime \prime}+v_{n s}{ }^{\prime \prime}+\left[k_{b}^{2}(z)-2 k_{b}(z)\{2 g(d) k(z)+i 2 g(a)(z)\}+\right. \\
\left.\mu_{b}+\delta(z)-k_{n}{ }^{2}\right] v_{n}(z)=0
\end{array}
$$

Adding and subtracting $k_{n b}^{2} v_{n}(z)$ to the left hand side of equation (8.6), we obtain

$$
\begin{aligned}
& v_{n b}^{\prime \prime}+v_{n s}{ }^{\prime \prime}+\left[k_{b}^{2}+m_{b}(z)-k_{n b}^{2}\right] v_{n}(z)+ \\
& \quad\left[2 k_{b}(z)\{2 g(d) k(z)+i \alpha(z)\}+\delta(z)\right] v_{n}(z)-\left\{k_{n}{ }^{2}-k_{b}{ }^{2}\right\} v_{n}(z)=0
\end{aligned}
$$


Subtracting equation (8.7) from equation (8.3), we obtain

$$
\begin{aligned}
v_{n s} "+ & {\left[k_{b}^{2}(z)+\mu_{b}(z)-k_{n b}{ }^{2}\right] v_{n s}(z) } \\
& =-\left\{2 k_{b}(\delta k(z)+i \alpha(z))+\delta(z)\right\} v_{n}(z)+\left(k_{n}{ }^{2}-k_{n b}{ }^{2}\right) v_{n}(z)
\end{aligned}
$$

Taking the complex conjugate of equation (8.3), we obtain

$$
\frac{d^{2} v_{n b}^{*}(z)}{d z^{2}}+\left(k_{b}^{2}(z)+\mu_{b}(z)-k_{x}^{2}\right) v_{n b}^{*}(z)=0
$$

Multiplying equation (8.8) by $v_{n b}{ }^{*}$ and equation (8.9) by $v_{n s}$ and subtracting we obtain

$$
\begin{aligned}
v_{n b}^{*} v_{n s}{ }^{\prime \prime}-v_{n s} v_{n b}{ }^{*}=- & {\left[2 k_{b}(z)\{\delta(z)+i \alpha(z)\}+\delta(z)\right] v_{n}(z) v_{n b}{ }^{*}(z) } \\
& +\left(k_{n}{ }^{2}-k_{n b}{ }^{2}\right) v_{n}(z) v_{n b}{ }^{*}(z)
\end{aligned}
$$

We now integrate over the entire depth. The left hand side then becomes equal to zero. The resulting equation is then,

$$
\begin{aligned}
\left(k_{n}^{2}-k_{n b}^{2}\right) & \int_{0}^{\infty} v_{n}(z) v_{n b}{ }^{*}(z) d z= \\
& \int_{0}^{\infty}\left[2 k_{b}(z)\{\delta k(z)+i \alpha(z)\}+\delta(z)\right] v_{n}(z) v_{n b}{ }^{*}(z) d z
\end{aligned}
$$

Using the first order approximation, $v_{n}(z)=v_{n b}(z)$, we obtain

$$
\begin{aligned}
\left(k_{n}^{2}-k_{n b}^{2}\right) & \int_{0}^{\infty} \frac{U_{n b}(z) U_{n b}{ }^{*}(z)}{\rho_{b}^{1 / 2}(z) \rho_{b}{ }^{1 / 2}(z)} d z= \\
& \int_{0}^{\infty} \frac{1}{\rho(z)}\left[2 k_{b}(z)\{\delta k(z)+i \alpha(z)\}+\delta(z)\right] U_{n b}(z) U_{n b}{ }^{*}(z) d z
\end{aligned}
$$


Since $U_{n}$ s are orthogonal, we obtain

$$
\frac{k_{n}^{2}-k_{n b}^{2}}{N_{n}}=\int_{0}^{\infty} \frac{1}{\rho(z)}\left[2 k_{b}(z)\{\delta(z)+i \alpha(z)\}+\delta(z)\right]\left|U_{n b}(z)\right|^{2} d z
$$

Let,

$$
k_{n}=k_{n b}+\delta k=k_{n b}+\left(\delta k_{n}\right)_{r}+i\left(\delta k_{n}\right)_{i}
$$

Then,

$$
k_{n}^{2}-k_{n b}^{2}=2 k_{n b}\left\{\left(\delta_{n}\right)_{r}+i\left(\delta_{n}\right)_{i}\right\}
$$

Substituting these relations in equation (8.13) we obtain the following integral equation for the unknown parameters.

$$
\begin{aligned}
& \frac{k_{n b}\left(\delta k_{n}\right)_{r}}{N_{n}}=\int_{0}^{\infty 1} \frac{1}{\rho_{b}(z)}\left\{k_{b}(z) \delta k(z)+\delta(z) / 2\right\}\left|U_{n b}\right|^{2} d z \\
& \frac{k_{n b}\left(\delta k_{n}\right)_{i}}{N_{n}}=\int_{0}^{\infty 1} \frac{1}{\rho_{b}(z)} k_{b}(z) \alpha k(z)\left|U_{n b}\right|^{2} d z
\end{aligned}
$$

If the experiment is performed at two frequencies $\delta k(z)$ and $\delta(z)$ can be seperated. The approach is the same as the one shown in Chapter 7 . We can recover the corrections to density, sound speed and attenuation in this manner. The similarity between the equations (8.16) and (8.17) and those used in connection with the deep water problem is evident. Therefore the methods suggested in Chapter 6 can be used to solve the above equations and obtain the geo-acoustic parameters of the bottom. 
$-228-$

\subsection{Inversion for sound speed using synthetic data}

Unlike the problem where the reflection coefficent at many angles of incidence are available as data, in this case the number of data points are limited by the number of modes. Further the modes have a decaying field in the bottom and therefore the penetration of the modes is small. The changes in the eigenvalues are affected by the sediment properties only in the top layers. If the source frequency is reduced then the modes penetrate deeper but the number of modes gets reduced, thereby reducing the number of data points available for inversion.

To test the validity of this method, the synthetic data were generated. A model for the ocean bottom was assumed and the modal eigenvalues determined numerically by using a search routine which searches for the location of the poles. For the guess model the modal eigenvalues were similarly obtained. The bottom was assumed to be lossless. Having thus obtained the difference in the modal eigenvalues inversion algorithm was used to reconstruct the bottom acoustic parameter[3]. In this case it was assumed that the density in the bottom was known leaving only the bottom sound speed to be determined.

To study the effect of the magnitude of the perturbation two cases were studied; one in which the perturbation was taken as $50 \mathrm{~m} / \mathrm{s}$ and the other in which it was $100 \mathrm{~m} / \mathrm{s}$. The exact and guess models are given in Figures 8-4 and 8-5.

The modal eigen values were computed at three different frequencies 50 $\mathrm{Hz}, 100 \mathrm{~Hz}$ and $200 \mathrm{~Hz}$. The data from the two frequencies $50 \mathrm{~Hz}$ and $100 \mathrm{~Hz}$ were then seperately used in an inversion algorithm. The results obtained are given in Figures 8-6 and 8-7. In order to increase the number of data points, the data from all three frequencies were combined and used in the inversion procedure. In this case we further made the assumption that the location of the sub-bottom is known. 


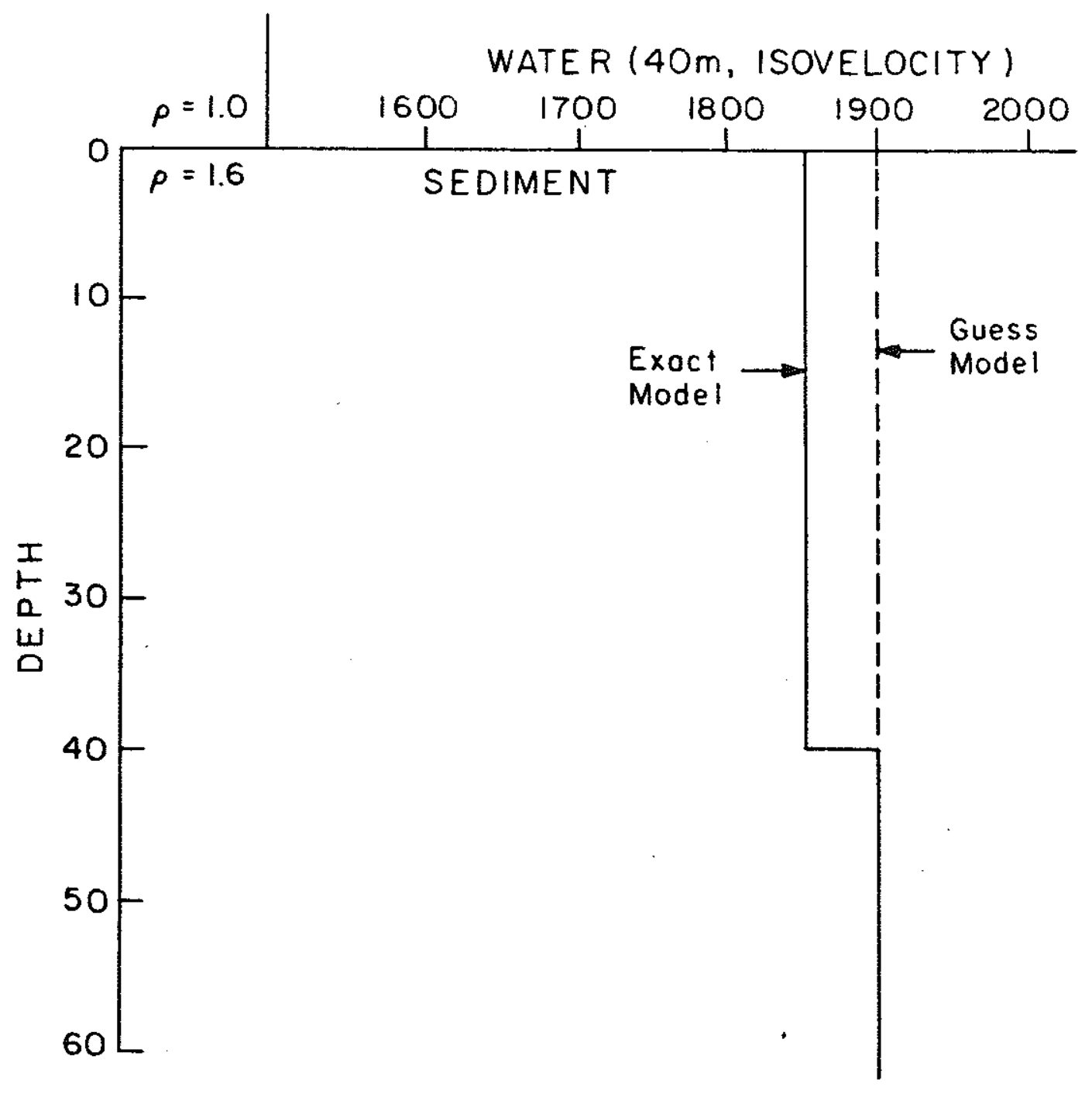

Figure 8-4: Exact and guess model - case(i) 


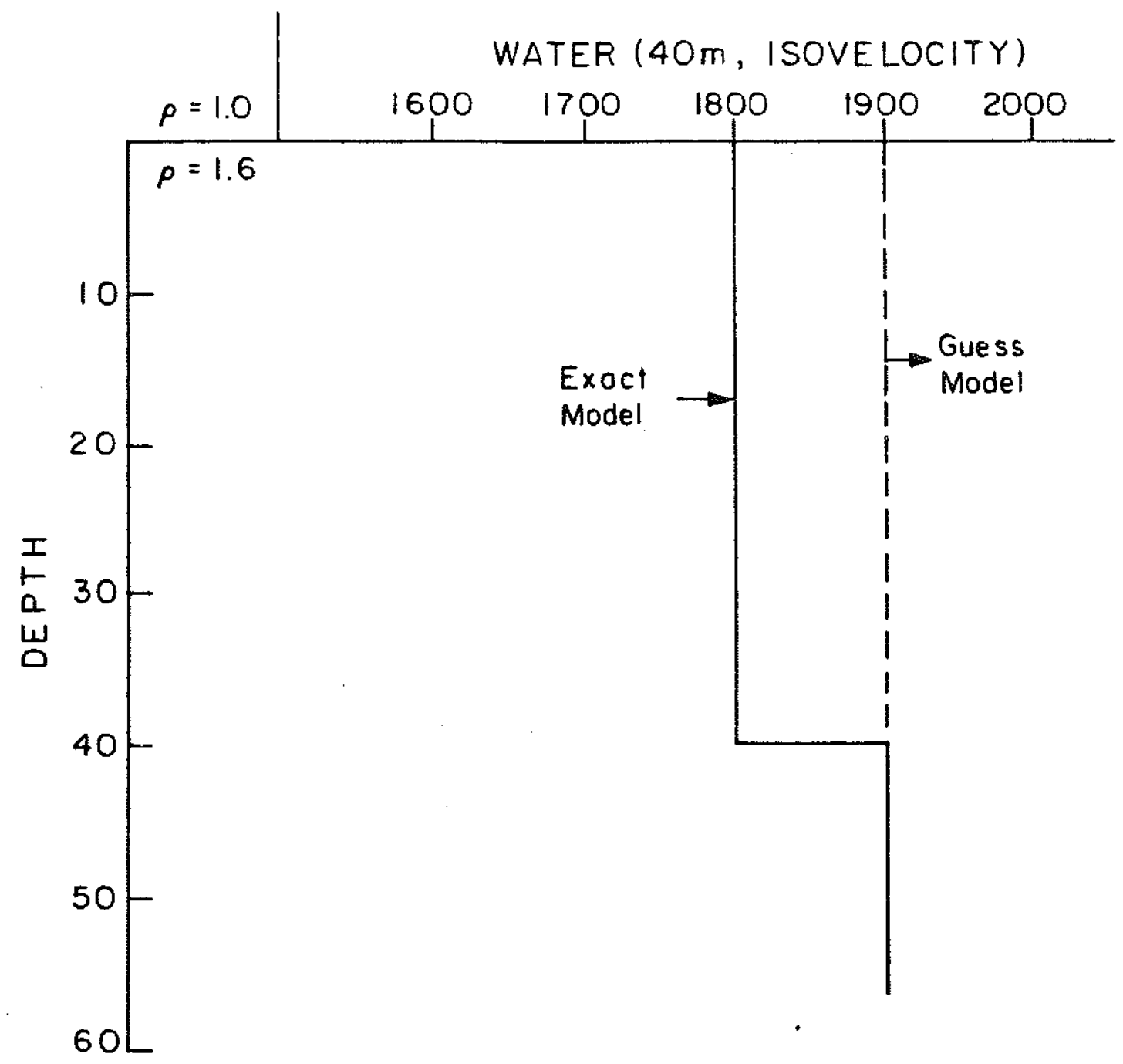

Figure 8-5: Exact and guess model - case(ii) 


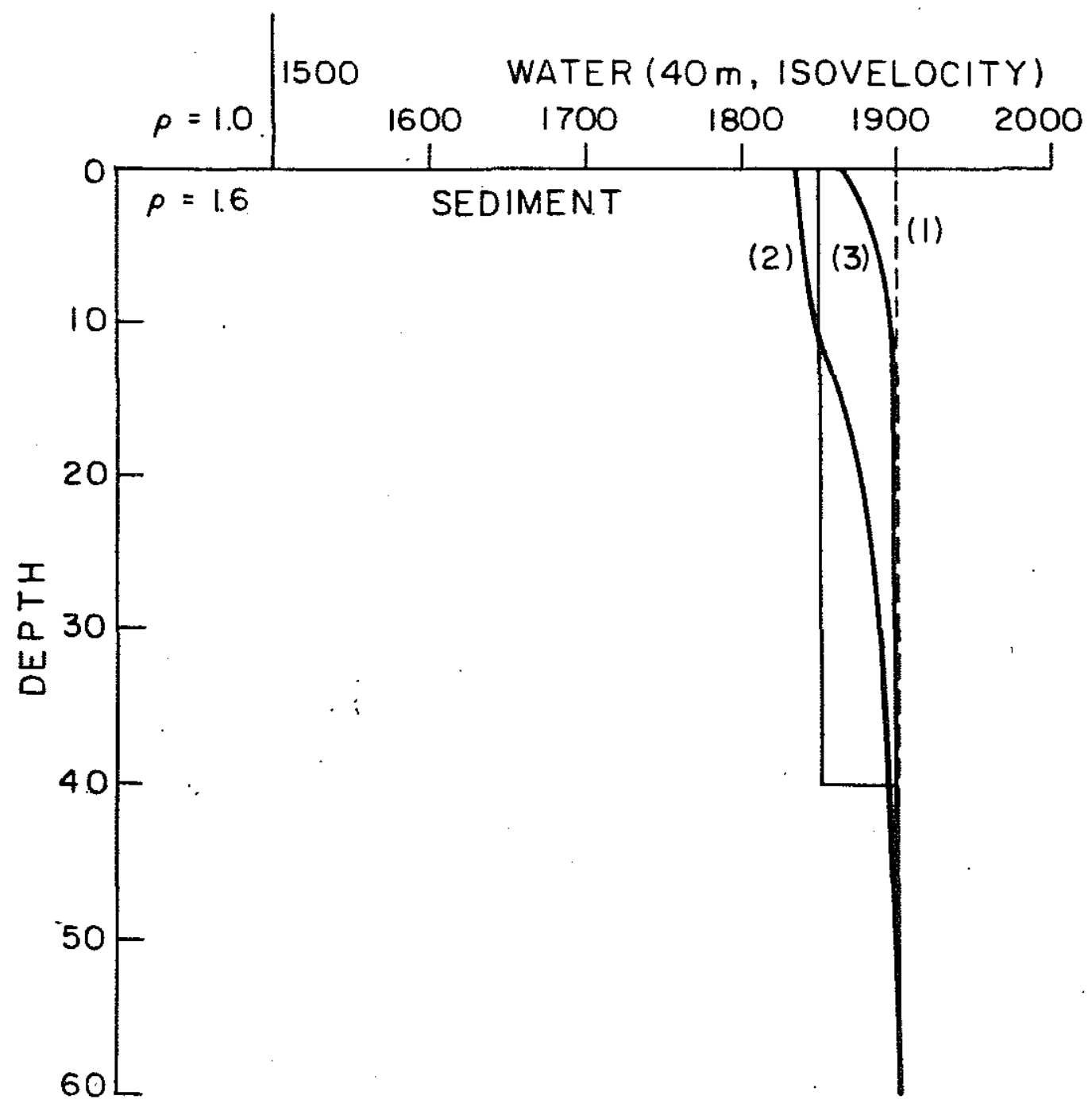

Figure 8-6: Reconstructed profile - case(i)

Frequencies $-50 \mathrm{~Hz}$ and $100 \mathrm{~Hz}$

1- Initial guess, 2- Reconstructed profile using $100 \mathrm{~Hz}$ data, 3 - Reconstructed profile using $50 \mathrm{~Hz}$ data 
$-232-$

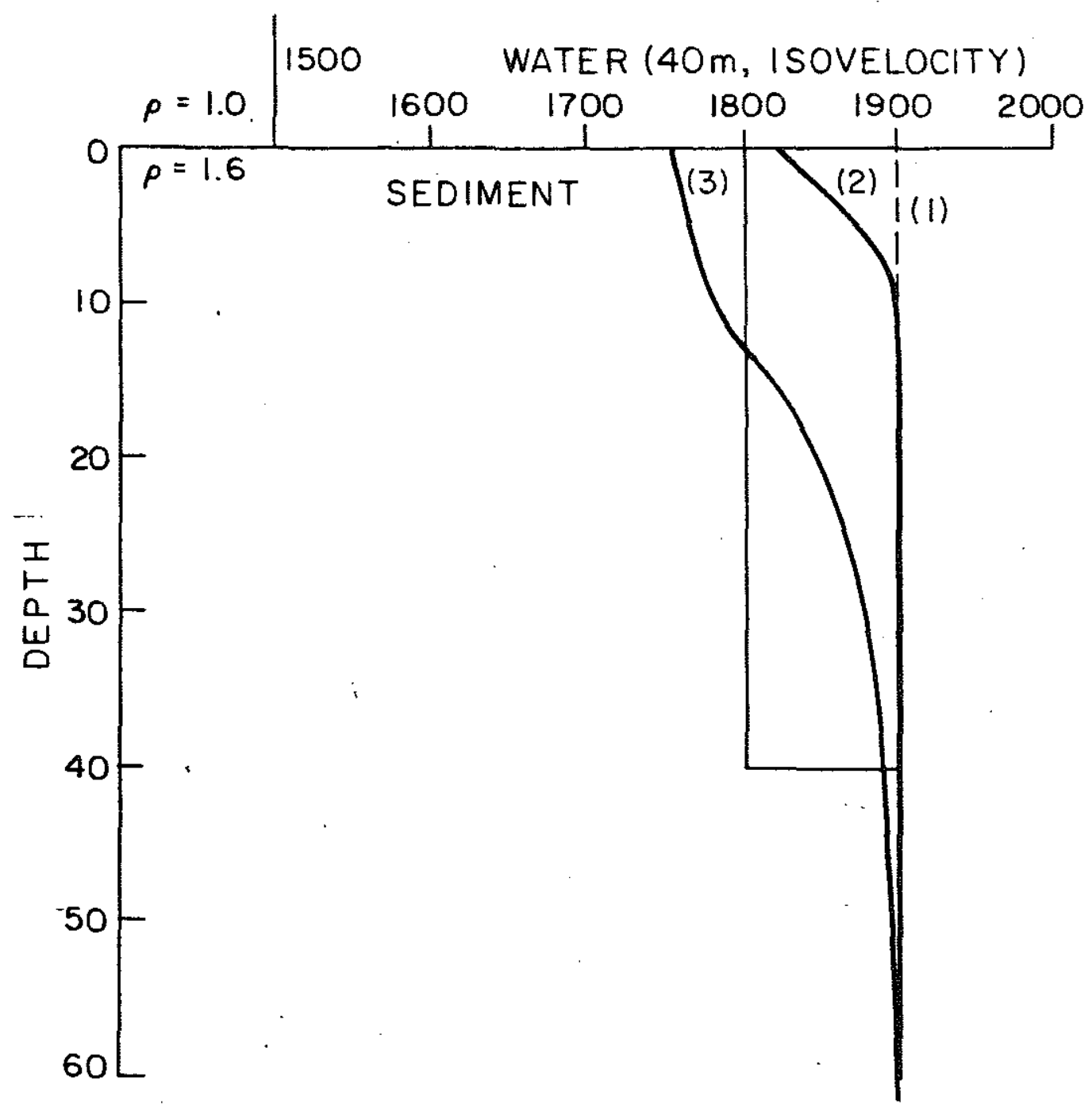

Figure 8-7: Reconstructed profile - case(ii)

frequencies- $50 \mathrm{~Hz}$ and $100 \mathrm{~Hz}$

1- Initial guess, 2- Reconstructed profile using $100 \mathrm{~Hz}$ data, 3- Reconstructed profile using $50 \mathrm{~Hz}$ data 


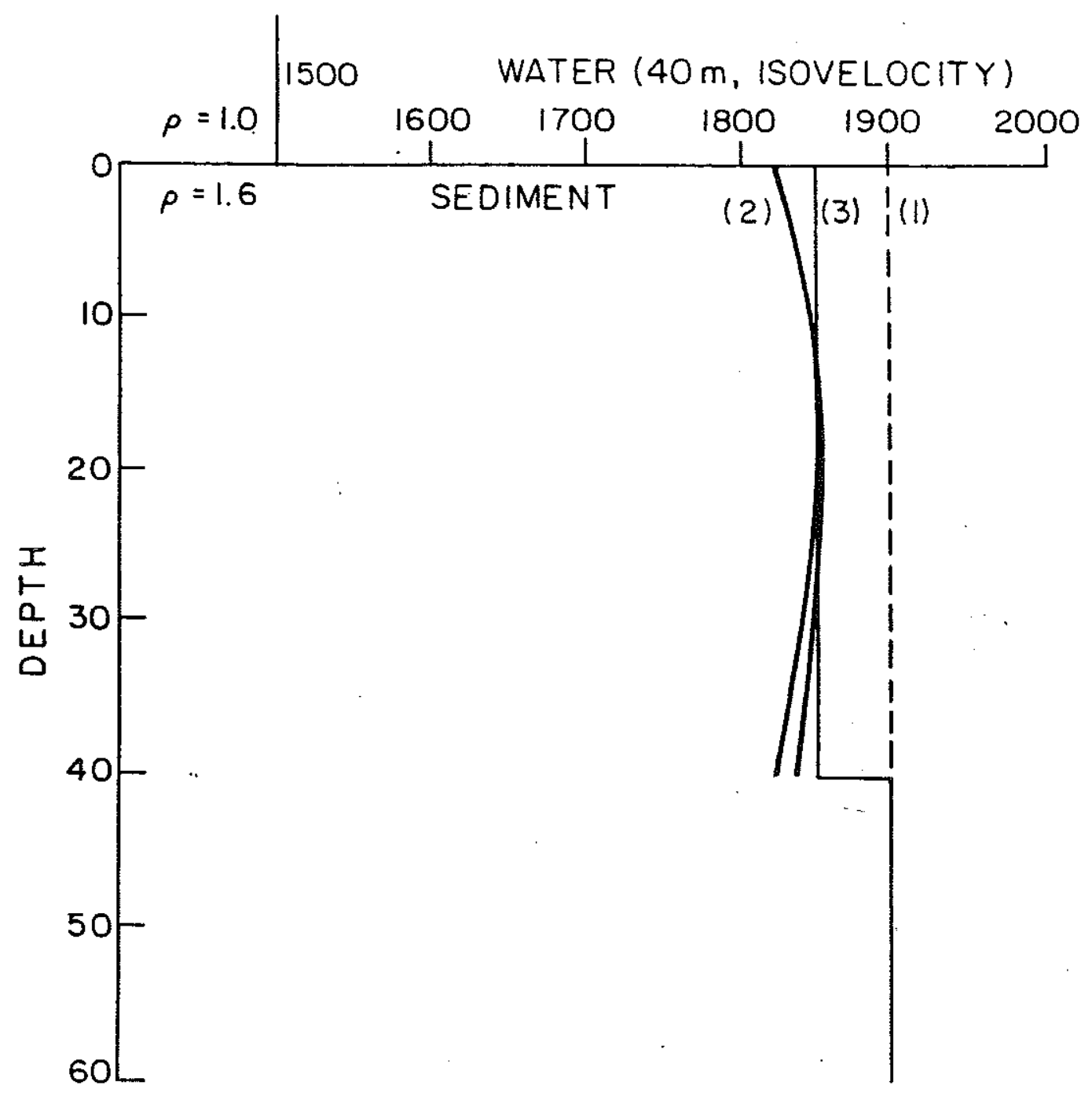

Figure 8-8: Reconstructed profile using data from all three frequencies- case(i)

1- Initial guess, 2- Reconstructed profile, 3. Reconstructed profile after one iteration 


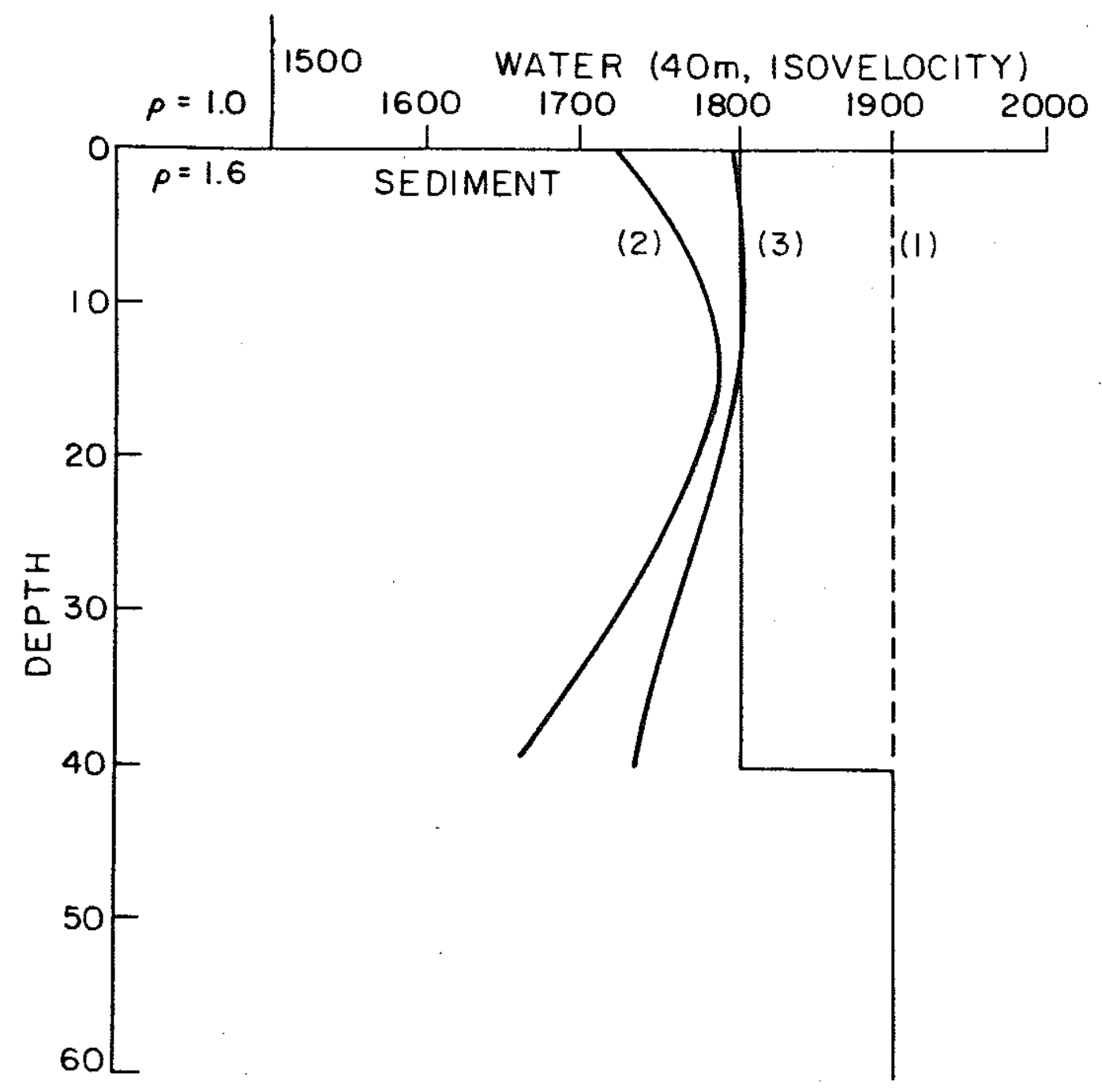

Figure 8-9: Reconstructed profile using data from all three frequencies- case(ii)

1- Initial guess, 2-Reconstructed profile, 3- Reconstructed profile after one iteration 
The inversion method was similar to the one described in the last chapter, namely that we looked for a smooth solution. The results are given in Figures 8-8and 8-9.

The following observations are made on the basis of results obtained,

1. In the case of high frequency, reconstruction of only the top layer is possible. At lower frequencies reconstruction to a deeper depth can be obtained.

2. When data obtained at three different frequencies are used as input data the situation improved considerably as more data points are available.

3. If we add more information to the scheme such as the speed in the subbottom and the location of this interface, the results of inversion are better.

\subsection{Inversion of real data}

The data obtained in the field experiment was processed and the modal eigen values determined at two frequencies $140 \mathrm{~Hz}$ and $220 \mathrm{~Hz}$. Inversion for the sound speed structure of the bottom was carried out using the method proposed earlier. The result obtained is shown in Figure 8-10. The profile for the bottom is similar to the one proposed by Hamilton[4] for sandy sediments.

Work on this approach to obtain the acoustic parameters of the bottom is continuing and a more detailed investigation will be necessary before any conclusions can be made on the efficacy of the proposed method. 
$-236-$

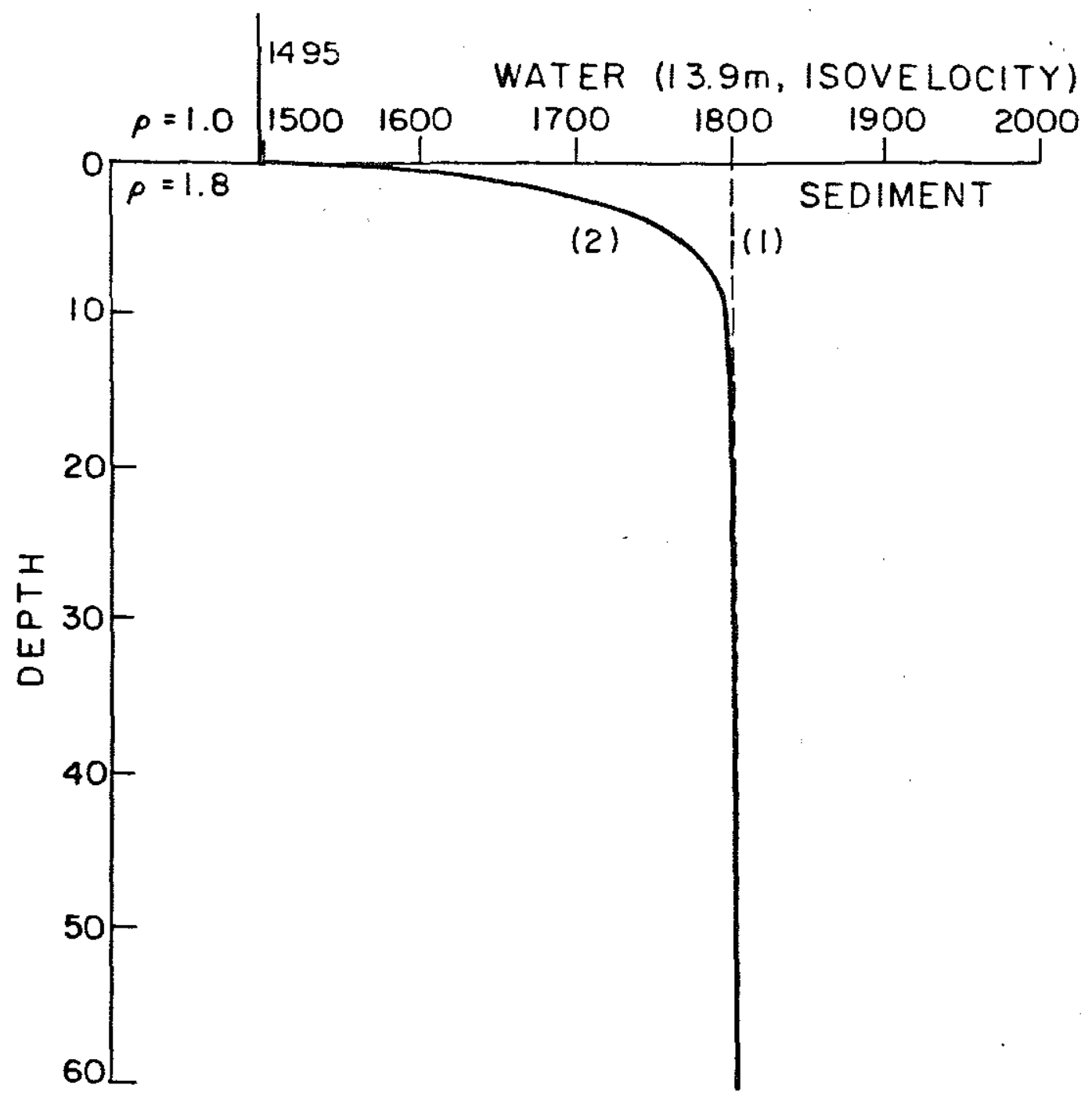

Figure 8-10: Inversion of real data

1- Initial guess, 2- Reconstructed profile 


\subsection{References}

1. G.V. Frisk and J.F. Lynch, "Shallow water waveguide characterisation using Hankel transform," J. Acoust. Soc. Am.,76, 205-216 (1984).

2. G.V. Frisk, J.F. LyDch, and J.A. Doutt, "Shallow water waveguide characterisation using Hankel transform - Preliminary results," J. Acoust. Soc. Am., 76(s1)S(84), (1884).

3. S.D. Rajan, M.S. Wengrovitz, J.F. Lynch, and G.V. Frisk, "A linear inverse scheme for the extraction of ocean bottom geoacoustic parameters from modal eigenvalues," J. Acoust. Soc. Am., 76(S1) S(94), (1984).

4. E.L. Hamilton, "Geoacoustic modelling of the sea noor, "J. Acoust. Soc. Am., 68(5), 1313-1340 (1980). 


\section{Chapter 9 Conclusions}

In this thesis, we developed a perturbation method for obtaining the profiles of the compressional wave speed, the compressional wave attenuation and density in marine sediments. We inferred these parameters using an inverse procedure that has as its input the plane wave reflection coefficent as function of angle of incidence at a fixed frequency. The Born approximation was applied to obtain a linear Fredholm integral equation of the first kind. A numerical method for solving the integral equation using a priori information on the smoothness of the solution was adopted and the inversion procedure tested with noisy and noise free synthetic data. In both cases the scheme yielded stable and acceptable results.

In Chapter 2 we studied the propagation of plane waves in marine sediments and developed an acceptable ocean bottom model. We noted that no acceptable theoretical model as yet exists to describe the variation of the attenuation coefficient at low frequencies in marine sediments. Further, we noted that dispersion of the propagating waves can be substantial for coarse sediments. This led us to propose a field experiment using a mono-chromatic source for determining the acoustic properties of the ocean bottom. In Chapter 3 we briefly described the experiment and enumerated the processing steps to be used to obtain the acoustic parameters.

In Chapter 4 we dealt with the forward problem and obtained a numerically stable propagator matrix algorithm to compute the plane wave reflection coefficient and the wave field in the sediment. An alternate method of solving the forward 
problem using a uniform asymptotic solution was also indicated in the chay ter.

In Chapter 5, we derived the linear Fredholm integral equation of the first kind for obtaining the attenuation coefficent profile for the sediment layer. The linear equation was obtained using the Born and Rytov approximations. We derived an expression for the region of validity of the Born approximation and showed that it is dependent on the magnitude and extent of the perturbation. A review of the methods available for the solution of this class of integral equation was made in Chapter 6 and an iterative method for solving non-linear integral equation indicated.

In Chapter 7 we established that the kernel of the non-linear integral equation is Frechet differentiable and that the Kernel obtained by the Born approximation is a Frechet kernel. We then arrived at the optimum aperture angle for input information by studying the region of validity of the Born approximation. We noted that this corresponds to pre-critical angles. We also showed that if the sound speed and density are known exactly, and only the attenuation coefficient is desired, then the frequency at which the experiment is conducted does not affect the reconstruction.

The inversion procedure to obtain the attenuation coefficent profile was demonstrated using synthetic data for different types of profiles including a discontinuous one. The solution was obtained by making an a priori assumption on the smoothness of the solution. The ability of the data to resolve fine details of the solution was studied by computing the Dirichlet resolving kernel using the resolving power theory of Backus and Gilbert. In the case of noise free data the method yielded adequate resolution. We also derived the resolving kernel when the unknown is a complex quantity and obtained an expression for the resolving kernel and a contamination term. The contamination term determines how the imaginary 
part of the solution affects the reconstruction of the real part and vice-versa.

We showed that the reconstruction of the attenuation coefficent is sensitive to errors in the sound speed and density and then developed and demonstrated a method where the errors are treated as additional unknowns in the inversion. However, when reconstruction of the sound speed is performed, the errors increase with frequency. Hence the accuracy of the reconstruction will be limited by the frequency at which the experiment is performed.

The effect of additive noise in the input data was investigated and in the case of the reconstruction of the attenuation profile with noisy data, we noted that the scheme is stable.

In Chapter 8, we derived the integral equation for obtaing the acoustic parameters of the ocean bottom from modal eigenvalues. This method is applicable to shallow water wave guides. We demonstrated the inversion using synthetic data and one set of data acquired in a field experiment. The preliminary results show promise and further work is continuing.

The foundations laid in this thesis provide a basis for future work in other areas some of which are presented in the next section.

\subsection{Suggestions for future work}

\subsubsection{Testing the validity with real data}

We used primarily synthetic data in this thesis. The validity of the scheme needs to be tested fully using real data. 


\subsubsection{Effect of shear}

In the development of the reconstruction scheme, we assumed the sediment to be a fluid. However, the sediment supports shear and part of the compressional wave energy is converted to shear. This will manifest itself as additional loss. The effect of shear on the reconstruction therefore needs investigation. Also possibility of extending the method to give information about shear is another aspect to be investigated.

\subsubsection{Shallow water problem}

Only very preliminary investigation has been done on the method proposed for reconstruction of acoustic parameters from modal eigenvalues. Further work needs to be done in this regard.

\subsubsection{Inversion from the point source pressure field}

The input information for the inversion scheme is the plane wave reflection coefficent while the measured quatity is the complex point source pressure field. The measured pressure field is processed to get the input data. Errors in input data as well as assumptions made in the processing of the data will affect the quality of the reflection coefficents obtained. For example it has been shown that one the sources of error in the Hankel transform operation is the variation in source height. A scheme which takes the measured pressure fields as data will be less prone to such processing errors and therefore the possibility of developing such a scheme needs investigation 


\section{Appendix A}

Consider the model in Figure (2-16). We now derive the Green's function which satisfies the following equation.

$$
\frac{d^{2} G}{d z^{2}}+\left(k^{2}(z)+\mu(z)-k_{x}{ }^{2}\right) G=\delta\left(z-z^{\prime}\right) \quad 0 \leq z, z^{\prime} \leq h
$$

where,

$$
\begin{aligned}
& k(z)=\frac{\omega}{C(z)} \\
& \mu(z)=\frac{\rho^{1 / 2}(z)}{2}\left\{\frac{\rho^{\prime}(z)}{\rho^{3 / 2}(z)}\right\}^{\prime} \\
& k_{x}=\frac{\omega}{C_{0}} \sin \theta_{0}
\end{aligned}
$$

Equation (A.1) can then be written as below.

$$
\frac{d^{2} G}{d z^{2}}+\omega^{2} Q^{2}(z) G=\delta\left(z-z^{\prime}\right) \quad 0 \leq z, z^{\prime} \leq h
$$

where

$$
Q^{2}(z)=\left\{\frac{1}{C^{2}(z)}+\frac{\mu(z)}{\omega^{2}}-\frac{\sin ^{2} \theta_{0}}{C_{0}^{2}}\right\}
$$

To solve for the Green's function, we note that the Green's function satisfies the homogeneous form of equation (A.2) when $z \neq z^{\prime}$. Therefore in the regions where $z \neq z^{\prime}$, we write the solution as the linear combination of the two independent 
solutions. When $Q(z)$ is a smoothly varying function and if there is only one turning point, the solution can be written in terms of the uniform asymptotic solutions. Let $g_{1}(z)$ and $g_{2}(z)$ be the two linearly independent solutions. Then,

$$
\begin{aligned}
& g_{1}(z)=Q^{-1 / 2}(z) \nu^{1 / 4}(z) A i(-\nu) \\
& g_{2}(z)=Q^{-1 / 2}(z) \nu^{1 / 4}(z) B i(-\nu)
\end{aligned}
$$

where,

$$
\begin{aligned}
& \nu(z)=\{3 / 2 \omega \xi(z)\}^{2 / 3} \\
& \xi(z)=\int_{z_{t}}^{z} Q(t) d t
\end{aligned}
$$

$z_{t}$ is the turning point i.e. $Q\left(z_{t}\right)=0 . A_{i}$ and $B_{i}$ are the Airy functions.

We now write the solutions in the two regions $0 \leq z<z^{\prime}$ and $z^{\prime}<z \leq h$ in terms of these solutions.

$$
\begin{array}{ll}
G\left(z, z^{\prime}\right)=A g_{1}(z)+B g_{2}(z) & 0 \leq z<z^{\prime} \\
G\left(z, z^{\prime}\right)=C g_{1}(z)+D g_{2}(z) & z^{\prime}<z \leq h
\end{array}
$$

These solutions must satisfy the boundary conditions at $z=0$ and $z=h$. Further the function $G\left(z, z^{\prime}\right)$ must be continuous at $z=z^{\prime}$. $d G / d z$, however, is discontinuous at $z=z^{\prime}$

In the region $z<0$, the region is homogeneous. The field in this region is an outgoing wave of the form $T_{0} \exp \left(i k_{z 0} z\right) . k_{z 0}$ is the vertical wave number in the region $z<0$. Applying boundary conditions of continuity of pressure and normal particle velocity at $z=0$, 


$$
\begin{aligned}
& A g_{1}(0)+B g_{2}(0)=T_{0} \\
& \left.\frac{1}{\rho(0)}\left\{A \rho^{1 / 2}(z) g_{1}(z)+B \rho^{1 / 2}(z) g_{2}(z)\right\}^{\prime}\right|_{0}=\frac{i k_{z 0}}{\rho_{0}} T_{0}
\end{aligned}
$$

We now assume that $\rho(z)$ and its first derivative are continuous across the interface. With this assumption, we obtain

$$
\frac{A}{B}=\frac{\rho_{0} g_{2}{ }^{\prime}(0)+i k_{z 0} \rho^{1 / 2}(0) g_{2}(0)}{\rho_{0} g_{1}{ }^{\prime}(0)+i k_{z 0} \rho^{1 / 2}(0) g_{1}(0)}
$$

We now write the solution in the region $z<z^{\prime}$ as follows.

$$
G\left(z, z^{\prime}\right)=B\left\{R_{s} g_{1}(z)+g_{2}(z)\right\}
$$

where $R_{s}=A / B$ and is given by the equation (A.9). Similarly by applying the boundary conditions at $z=h$, we obtain for $z>z^{\prime}$,

$$
G\left(z, z^{\prime}\right)=C\left\{g_{1}(z)+R_{b} g_{2}(z)\right\}
$$

where

$$
R_{b}=\frac{\rho_{0} g_{1}{ }^{\prime}(h)-i k_{z 2} \rho^{1 / 2}(h) g_{1}(h)}{\rho_{0} g_{2}{ }^{\prime}(h)-i k_{z 2} \rho^{1 / 2}(h) g_{2}(h)}
$$

Since $G\left(z, z^{\prime}\right)$ is continuous at $z=z^{\prime}$, the solution is written as below.

$$
\begin{aligned}
& G\left(z, z^{\prime}\right)=C_{1} \psi_{2}\left(z^{\prime}\right) \psi_{1}(z) \quad z<z^{\prime} \\
& G\left(z, z^{\prime}\right)=C_{1} \psi_{1}\left(z^{\prime}\right) \psi_{2}(z) \quad z^{\prime}<z
\end{aligned}
$$

where, 


$$
\begin{aligned}
& \psi_{1}(z)=R_{s} g_{1}(z)+g_{2}(z) \\
& \psi_{2}(z)=g_{1}(z)+R_{b} g_{2}(z)
\end{aligned}
$$

$C_{1}$ is a constant and is equal to $1 / W\left\{\psi_{1}(z), \psi_{2}(z)\right\}$. The Wronksian $W\left\{\psi_{1}(z), \psi_{2}(z)\right.$ is given by

$$
W\left(\psi_{1}(z), \psi_{2}(z)\right)=\psi_{2}(z) \psi_{1}^{\prime}(z)-\psi_{1}(z) \psi_{2}^{\prime}(z)
$$

Substituting for $\psi_{1}(z)$ and $\psi_{2}(z)$ from equations (A.14) and (A.15), we obtain

$$
W\left(\psi_{1}(z), \psi_{2}(z)\right)=\frac{\pi}{\omega} \frac{1}{1-R_{s} R_{b}}
$$

The solution for the Green's function is therefore,

$$
\begin{array}{rll}
G\left(z, z^{\prime}\right) & =\frac{\pi}{\omega} \frac{1}{1-R_{s} R_{b}}\left\{\psi_{2}\left(z^{\prime}\right) \psi_{1}(z)\right\} & z<z^{\prime} \\
G\left(z, z^{\prime}\right) & =\frac{\pi}{\omega} \frac{1}{1-R_{s} R_{b}}\left\{\psi_{1}\left(z^{\prime}\right) \psi_{2}(z)\right\} & z^{\prime}<z
\end{array}
$$

If there are no turning points, then the uniform asymptotic solution reduces to the WKB solution. Then,

$$
\begin{aligned}
& g_{1}(z)=\frac{1}{(\omega Q(z))^{1 / 2}} \exp \left(i \omega \int^{z} Q(t) d t\right) \quad z<z^{\prime} \\
& g_{2}(z)=\frac{1}{(\omega Q(z))^{1 / 2}} \exp \left(-i \omega \int^{z} Q(t) d t\right) \quad z<z^{\prime}
\end{aligned}
$$

With this solution for $g_{1}(z)$ and $g_{2}(z)$, we can show that $R_{s}$ and $R_{b}$ look like the Rayleigh reflection coefficents. Let us consider $R_{s}$. 


$$
g_{1}^{\prime}(0)={\frac{Q^{1 / 2}}{\omega^{1 / 2}}}^{1 / \omega}\left\{\frac{Q^{\prime}(0)}{2 Q^{2}(0)}\right\}
$$

For a smoothly varying medium $\left\{Q^{\prime}(z) / Q^{2}(z)\right\} \ll 1$ and in the frequency range we are considering this term is negligible. Therfore,

$$
g_{1}^{\prime}(0)=i\{\omega Q(0)\}^{1 / 2}
$$

Similarly,

$$
g_{2}^{\prime}(0)=-i\{\omega Q(0)\}^{1 / 2}
$$

But $\omega Q(0)=k_{z}(0)$ where $k_{z}(0)=k^{2}(0)-k_{x}{ }^{2}$. Using these relations in equation (A.2) we obtain,

$$
\frac{A}{B}=\frac{\rho_{0} k_{z}(0)-\rho(0) k_{z 0}}{\rho_{0} k_{z}(0)+\rho(0) k_{z 0}}
$$

Compare this with the equation for the Rayleigh reflection coefficent $R$ between two medium with densities $\rho_{0}$ and $\rho_{1}$ and sound speeds $C_{0}$ and $C_{1}$. For an incident wave in the medium with parameters $\rho_{1}$ and $C_{1}$

$$
R=\frac{\rho_{0} k_{z 1}-\rho_{1} k_{z 0}}{\rho_{0} k_{z 1}+\rho_{1} k_{z 0}}
$$




\section{Appendix B}

In this appendix we indicate how errors in the acoustic parameters of the subbottom can be dealt with. In this we follow the method of Coen[1].

Consider the case where the error is in the value of the attenuation coefficient in the subbottom. We express the true value of the attenuation coefficent as a sum of a background value and a small error term.

$$
\alpha_{2}=\alpha_{2 b}+\delta \alpha
$$

Proceeding in exactly the same manner as in Chapter 5 , an integral equation is obtained.

$$
\begin{array}{r}
2 i k_{z 0}\left\{R_{b}\left(k_{x}\right)-R\left(k_{x}\right)\right\}=\int_{0}^{h} \alpha(z) P_{b}^{2}\left(k_{x}, z\right) d z+ \\
\int_{h}^{\infty} \delta \alpha P_{b}^{2}\left(k_{x}, z\right) d z
\end{array}
$$

For discrete values of angle we write

$$
\begin{array}{r}
d_{n}=\int_{0}^{h} \alpha(z) P_{b n}{ }^{2}(z) d z+ \\
\int_{h}^{\infty} \delta \alpha P_{b n}{ }^{2}(z) d z
\end{array}
$$

Since the unknown $\delta \alpha$ is a constant it is taken out of the integral and the following equation obtained. 


$$
d_{n}=\int_{0}^{h} \alpha(z) P_{b n}^{2}(z) d z+\delta \alpha I_{n}
$$

where

$$
I_{n}=\int_{h}^{\infty} P_{b n}^{2}(z) d z
$$

At some other angle we write

$$
d_{m}=\int_{0}^{h} \alpha(z) P_{b m}^{2}(z) d z+\delta \alpha I_{m}
$$

Eliminating $\delta \alpha$ from these two equations the following relation is obtained.

$$
d_{n} I_{m}-d_{m} I_{n}=\int_{0}^{h} \alpha(z)\left\{I_{m} P_{b n}{ }^{2}(z)-I_{n} P_{b m}{ }^{2}(z)\right\} d z
$$

For each data point $1, \ldots, n$, the contribution from the second term is subtracted out by using the $(n+1)$ th data point as shown above. The equations are now solved for $\alpha(z)$ as before. Having obtained $\alpha(z), \delta \alpha$ is then computed. Error in other parameters can be treated in a similar manner. 